\title{
Th-U series radionuclides in the characterization of geothermal reservoirs (Bruchsal, SW Germany)
}

\author{
Dissertation \\ zur Erlangung des mathematisch-naturwissenschaftlichen Doktorgrades \\ „Doctor rerum naturalium“ \\ der Georg-August-Universität Göttingen \\ im Promotionsprogramm Geosciences \\ der Georg-August University School of Science (GAUSS)
}

vorgelegt von

Lena Kölbel

aus Karlsruhe

Göttingen 2020 


\section{Betreuungsausschuss:}

Dr. Bettina Wiegand

Prof. Dr. Martin Sauter
Abteilung Angewandte Geologie, Georg-August-Universität Göttingen

Abteilung Angewandte Geologie, Georg-August-Universität Göttingen

\section{Mitglieder der Prüfungskommission:}

Referent: $\quad$ Prof. Dr. Martin Sauter

Korreferent: Prof. Dr. Thorsten Schäfer

Weitere Mitglieder der Prüfungskommission:

Dr. Bettina Wiegand

Dr. Iulia Ghergut

Prof. Dr. Andreas Pack

Prof. Dr. Volker Thiel
Abteilung Angewandte Geologie, Georg-August-Universität Göttingen

Institut für Angewandte Geowissenschaften, Friedrich-Schiller-Universität Jena

Abteilung Angewandte Geologie, Georg-August-Universität Göttingen

Abteilung Angewandte Geologie, Georg-August-Universität Göttingen

Abteilung Isotopengeologie,

Georg-August-Universität Göttingen

Abteilung Geobiologie,

Georg-August-Universität Göttingen 


\section{Abstract}

A sophisticated analysis of naturally occurring nuclides has the potential to support geothermal energy exploitation during various stages of a project's life cycle. One possible methodological approach is based on the detection of Th-U decay series disequilibria which are caused by a relatively rapid exchange of isotopes between fluid and rock within the reservoir section resulting in a fractionation of daughter and parent nuclides.

In deep geothermal systems, natural radionuclides exist in both the fluid phase and the adjoining solids. Since the fluid phase is extremely sensitive to radionuclide exchanges, it displays the extent of radioactive disequilibrium very evidently. Modelling of these radioactive disequilibria can provide site-specific information of the long-term migratory behavior of radionuclides which might be useful for the sustainable management of geothermal reservoirs. Furthermore, the understanding of the Th-U series nuclides behavior in geothermal systems enables the plant operators to handle radionuclide-bearing mineral precipitations which are known from surface installation parts. But even in early stages of geothermal projects, the examination and assessment of radionuclide signatures of rocks can have a practical benefit for geothermal reservoir engineering.

Only a few studies dealing with the tangible benefit of using radionuclide-based methods for deep geothermal projects are published. To shorten this gap in knowledge, a radiochemical monitoring system was realized at Bruchsal in the framework of the ANEMONA research project. The test site is located at the eastern main boundary fault of the Upper Rhine Graben in SW-Germany. A comprehensive dataset was generated by frequent, long-term fluid sampling and laboratory analyses. In addition, the Bruchsal reservoir rock was intensively examined based on drill cuttings from both the injection and production well. The results and their application are presented in three individual studies.

The first part of this thesis deals with a new approach to identify permeable fractures or at least formerly permeable fractures based on specific radionuclide signatures of rocks. Alpha and gamma spectrometry surveys of the Bruchsal drill cuttings delivered specific activities of the Th-U series nuclides. It is shown that the reservoir section at Bruchsal is defined by a positive ${ }^{226} \mathrm{Ra}$ anomaly in deeper borehole sections. A petrographic alteration study is performed on the rock material to determine links between the hydrothermal processes in the reservoir and the related accumulation of radium. Possible fractured zones are identified by the evaluation of $\mathrm{Th} / \mathrm{Ra}$ and $\mathrm{Ra} / \mathrm{K}$ ratios which are sensitive to changes in the ${ }^{226} \mathrm{Ra}$ concentration. The results are compared with the conventional well logs performed in the GB2 well.

The radiochemical analysis of the Bruchsal $\mathrm{NaCl}$ brine display radioactive disequilibria within the natural decay series caused by the preferred solution of radium isotopes $\left({ }^{228} \mathrm{Ra},{ }^{226} \mathrm{Ra},{ }^{224} \mathrm{Ra},{ }^{223} \mathrm{Ra}\right)$ 
having activities by far exceeding those of their thorium progenitors. Based on the observed radioactive disequilibria, water-rock interactions are examined in depth to evaluate their effects on radionuclide transport in the geothermal reservoir. The results are documented in the second part of this study. The mathematical treatment is based on Ku et al. (1992) whose water-rock interaction model allows a detailed analysis of physico-chemical reactions as well as the study of advective transport phenomena. The modelling part of this study is focused on radium isotopes since their range in half-lives and their interrelation in the respective decay chain allows the determination of water-rock interaction rates across different timescales. It is shown that diffusion along microfractures is an essential step allowing the recoiled atoms to enter the flow system and causing a significant fractionation of $\mathrm{Ra}$ isotopes.

In the third part of the thesis, an integrative approach to estimate site-specific fracture dimensions is introduced by means of a ${ }^{222} \mathrm{Rn}$ mass balance approach. Here, radon acts as a natural radiotracer whose activity in the geothermal fluid is mainly controlled by the diffusional flux from fracture surfaces, but also by the fracture geometry. Integral hydraulic parameters of the Bruchsal reservoir are obtained from the interpretation of pumping tests. Therefore, the aquifer test analysis tool AQTESOLV® is employed. The assessment of the fracture dimensions assumes an equivalent fracture. Thus, hydraulic parameters of a multi-fracture system are approximated using a single fracture, the so-called equivalent fracture. This approach offers the estimation of fracture parameters such as aperture, surface, etc. by using ${ }^{222} \mathrm{Rn}$. However, a certain ambiguity of the results cannot be ruled out. 


\section{Kurzfassung}

Eine detaillierte Analyse natürlich vorkommender Radionuklide hat das Potential die Nutzbarmachung der Tiefengeothermie in verschiedenen Projektphasen zu unterstützen. Der methodische Ansatz basiert dabei auf der Detektion radioaktiver Ungleichgewichte, welche durch einen vergleichsweisen schnellen Isotopenaustausch zwischen Fluid und Gestein im Reservoir verursacht werden und zu einer Fraktionierung zwischen Tochter- und Mutternukliden führen.

In tiefen geothermischen Systemen kommen natürliche Radionuklide sowohl in der fluiden Phase als auch im angrenzenden Gestein vor. Radioaktive Ungleichgewichte zeigen sich dabei besonders deutlich im Fluid, da dieses sehr leicht auf Austauschprozesse der Radionuklide reagiert. Durch die Modellierung dieser radioaktiven Ungleichgewichte können standort-spezifische Informationen über das langfristige Migrationsverhalten der Radionuklide gewonnen werden und kann damit für die nachhaltige Bewirtschaftung geothermischer Reservoire vorteilhaft sein. Überdies ermöglicht es den Anlagenbetreibern die fachgerechte Handhabung radionuklidhaltiger Mineralausfällungen wie sie aus obertägigen Anlagenteilen bekannt sind. Darüber hinaus kann bereits parallel zu Bohrarbeiten die Untersuchung und Bewertung von Radionuklid-Signaturen der Gesteine einen praktischen Nutzen für die Erschließung geothermischer Reservoire aufweisen.

Es wurden in der Vergangenheit vergleichsweise wenige Studien publiziert, die sich mit dem konkreten Nutzen der Anwendung radionuklidbasierter Methoden für Tiefengeothermie-Projekten befassen. Um diese Informationslücke zu verkürzen, wurde im Rahmen des Forschungsprojekts ANEMONA am Geothermiestandort Bruchsal ein radiochemisches Monitoringsystem installiert. Der Untersuchungsstandort befindet sich am östlichen Grabenrad des Oberrheingrabens in Südwestdeutschland. Durch regelmäßige und langwährende Probenahmen zusammen mit Laboranalysen geothermischer Fluide konnte ein um fangreicher Datensatz generiert werden. Zusätzlich wurden Bohrkleinproben aus dem Bruchsaler Reservoirgestein der Injektions- und Produktionsbohrung untersucht. Die Ergebnisse und deren Anwendung werden im Rahmen dieser Arbeit in drei eigenständigen Manuskripten vorgestellt.

Im ersten Teil dieser Arbeit wird eine neue Methode zur Identifikation von Bruchzonen mit heute oder zumindest vormals aktiver Fluidzirkulation anhand spezifischer Radionuklid-Signaturen im Gestein vorgestellt. Die spezifischen Aktivitäten der natürlichen Radionuklide wurden hierfür mittels Alpha- und Gammaspektrometrie am Bohrklein ermittelt. Es wird gezeigt, dass der Reservoirbereich durch eine positive ${ }^{226} \mathrm{Ra}$-Anomalie in den tieferen Bohrlochabschnitten gekennzeichnet ist. Ein möglicher Zusammenhang zwischen den hydrothermalen Vorgängen im Reservoir und der Anreicherung von Radium wird durch eine petrographische Alterationsstudie des Bohrkleins untersucht. Potenziell aktive Kluftzonen werden durch die Bewertung von Th/Ra und Ra/K-Verhältnissen des Gesteins identifiziert, die deutlich auf die Schwankungen der ${ }^{226} \mathrm{Ra}$-Konzentration 
reagieren. Die Ergebnisse wurden mit konventionellen Bohrlochmessungen der GB2 verglichen.

Die radiochemischen Untersuchungen der Bruchsaler NaCl-Sole zeigen radioaktive Ungleichgewichte innerhalb der natürlichen Zerfallsreihe, welche auf die bevorzugte Lösung der Radiumisotopen $\left({ }^{228} \mathrm{Ra},{ }^{226} \mathrm{Ra},{ }^{224} \mathrm{Ra}\right.$, $\left.{ }^{223} \mathrm{Ra}\right)$ zurückzuführen sind. Hierbei liegen die Ra-Aktivitäten weit über den Aktivitäten ihrer Thorium-Mutternuklide. Darauf aufbauend wurden Wasser-Gesteins-Interaktionsprozesse anhand der beobachteten radioaktiven Ungleichgewichte untersucht und ihre Auswirkungen auf den Radionuklidtransport im geothermischen Reservoir bewertet. Der mathematische Ansatz beruht auf Ku et al. (1992), dessen Wasser-Gesteins-Interaktionsmodell die detaillierte Betrachtung der physikalisch-chemischen Reaktionen sowie advektiver Transportvorgänge erlaubt. Das im Rahmen dieser Arbeit entwickelte Modell konzentriert sich auf die Verwendung von RaIsotopen, die aufgrund ihrer unterschiedlichen Halbwertszeiten und ihrer Stellung in den Zerfallsreihen die Bestimmung von Interaktionsraten über verschiedene Zeitskalen hinweg gestatten.

Im dritten Teil der Arbeit wird eine integrative Herangehensweise zur Abschätzung standortspezifischer Kluftgrößen basierend auf einem Massenbilanzierungsansatz von ${ }^{222} \mathrm{Rn}$ vorgestellt. Radon tritt dabei als natürlicher Radiotracer auf, dessen Aktivität im geothermischen Fluid maßgeblich durch den diffusiven Fluss von Kluftflächen sowie durch die Kluftgeometrie bestimmt sind. Relevante hydraulische Parameter des Bruchsaler Reservoirs wurden durch die Auswertung von Pumpversuchen ermittelt. Hierfür wurde die Software AQTESOLV® verwendet. Für die Abschätzung der Kluftgrößen kommt ein Äquivalentkluft-Modell zum Einsatz. Dabei werden die hydraulischen Parameter eines Mehrkluftsystems näherungsweise von einer einzelnen Kluft, der so genannten Äquivalentkluft, abgebildet. Dieser Ansatz erlaubt die Bewertung von Kluftgrößen wie Apertur, Oberfläche, etc. mittels ${ }^{222} \mathrm{Rn}$. Hier ist festzuhalten, dass mehrdeutige Ergebnisse nicht auszuschlieBen sind. 


\section{Acknowledgements}

First and foremost, my sincere thanks to my advisors Dr. Bettina Wiegand and Prof. Dr. Marin Sauter for the interesting research opportunity, and their tireless support in multiple ways. Without their guidance, inspiring discussions, and constant feedback this work would not have been achievable.

Likewise, many thanks to the further members of the thesis committee: Prof. Dr. Thorsten Schäfer (also for acting as the second reviewer), Prof. Dr. Andreas Pack, Prof. Dr. Volker Thiel, and Dr. Iulia Ghergut.

I would also like to thank the colleagues in the Geoscience Centre, especially Dr. Ulrich Maier who provided helpful insight into multicomponent reactive transport modeling.

I greatly appreciate the support received through Dr. Michael Bolz and his team in the laboratory of EnBW Kernkraft GmbH. Special thanks to Georgios Gkogkidis who has always supported me with his analytical expertise and knowledge.

Many thanks to EnBW Energie Baden-Württemberg AG who generously provide access to samples and data, I highly benefited from. I would particularly like to highlight the colleagues of the EnBW research and innovation department for their professional input in various ways.

My sincere thanks also go to the colleagues form the Institute for Nuclear Waste Disposal (INE), especially Prof. Dr. Thorsten Schäfer for valuable discussions and encouragement.

I am grateful to Dr. Jörn Bartels (AQUASOIL, Berlin) for enlightening conversations on hydraulic reservoir modeling.

This research was supported by German Federal Ministry for Economic Affairs and Energy (BMWi) who funded the joint project "ANEMONA - Plant monitoring as a key technology for the successful operation of geothermal power plants in Germany". Many thanks to all ANEMONA colleagues for their collaborative work.

Last but not the least, I would like to thank my family and friends for their continuous support and understanding. Special thanks to my husband for many stimulating discussions and encouraging words and to our lovely daughters for their (mostly) patient assistance. 


\section{List of Symbols}

\begin{tabular}{|c|c|c|}
\hline Symbol & Parameter & SI units \\
\hline$A$ & Specific activity of a dissolved radionuclide & $\mathrm{Bq} \mathrm{kg}-1$ \\
\hline$A^{*}$ & $\begin{array}{l}\text { Specific activity of a dissolved radionuclide originated from diffusional } \\
\text { flux }\end{array}$ & $\mathrm{Bq} \mathrm{kg-1}$ \\
\hline$A_{a}$ & Specific activity of an adsorbed radionuclide & $\mathrm{Bq} \mathrm{kg}{ }^{-1}$ \\
\hline $\bar{A}$ & Specific activity of a radionuclide in solids & $\mathrm{Bq} \mathrm{kg-1}$ \\
\hline C & Concentration of a dissolved radionuclide & atoms $\mathrm{kg}^{-1}$ \\
\hline$C_{a}$ & Concentration of an adsorbed radionuclide & atoms $\mathrm{kg}^{-1}$ \\
\hline$C^{i}$ & Initial concentration of a dissolved radionuclide & atoms $\mathrm{kg}^{-1}$ \\
\hline$D_{L}$ & Hydrodynamic dispersion coefficient & $\mathrm{m}^{2} \mathrm{~s}^{-1}$ \\
\hline$D_{m}$ & Diffusion coefficient of a radionuclide & $\mathrm{m}^{2} \mathrm{~s}^{-1}$ \\
\hline$F_{i}$ & Diffusional flux of a nuclide into larger fractures & $\mathrm{Bq} \mathrm{m}^{-2}$ \\
\hline$e_{R a}$ & Radium recoil efficiency relative to ${ }^{222} \mathrm{Rn}$ & - \\
\hline$\varepsilon_{i}$ & Recoil efficiency for nuclide $i$ & - \\
\hline$\phi$ & Porosity of the aquifer & - \\
\hline$h_{f}$ & Fracture height & $\mathrm{m}$ \\
\hline$k_{1}$ & First-order adsorption rate constant & $\mathrm{s}^{-1}$ \\
\hline$k_{2}$ & First-order desorption rate constant & $\mathrm{s}^{-1}$ \\
\hline$k_{p}$ & First-order precipitation rate constant & $\mathrm{s}^{-1}$ \\
\hline$k_{w}$ & First-order dissolution rate constant & $\mathrm{s}^{-1}$ \\
\hline K & Dimensionless distribution coefficient & - \\
\hline$K_{d}$ & Distribution coefficient & $\mathrm{L} \mathrm{kg}^{-1}$ \\
\hline$L$ & Diffusion length & $\mathrm{m}$ \\
\hline$L_{e f f}$ & Effective diffusion length & $\mathrm{m}$ \\
\hline$L_{f}$ & Fracture length & $\mathrm{m}$ \\
\hline$\lambda$ & Radioactive decay constant of radionuclide & $\mathrm{s}^{-1}$ \\
\hline$M_{b}$ & Mass of brine & $\mathrm{kg} \mathrm{m}^{-3}$ \\
\hline$M_{r}$ & Mass of rock & $\mathrm{kg} \mathrm{m}^{-3}$ \\
\hline$P$ & Supply rate of radionuclide to brine & $\mathrm{Bq} \mathrm{kg}{ }^{-1}$ \\
\hline$P_{d}$ & Supply rate of radionuclide to brine by desorption & $\mathrm{Bq} \mathrm{kg}^{-1}$ \\
\hline$P_{r}$ & Supply rate of radionuclide to brine by alpha recoil & $\mathrm{Bq} \mathrm{kg}^{-1}$ \\
\hline$P_{r}^{*}$ & $\begin{array}{l}\text { Supply rate of radionuclide to brine by alpha recoil in consideration of } \\
\text { diffusional flux }\end{array}$ & $\mathrm{Bq} \mathrm{kg}-1$ \\
\hline$P_{w}$ & Supply rate of radionuclide to brine by dissolution process & $\mathrm{Bq} \mathrm{kg}{ }^{-1}$ \\
\hline$Q$ & Supply rate of radionuclide by water flow & $\mathrm{Bq} \mathrm{kg-1}$ \\
\hline$r_{i}$ & Recoil distance of nuclide $i$ & $\mathrm{~m}$ \\
\hline
\end{tabular}




\begin{tabular}{|c|c|c|}
\hline Symbol & Parameter & SI units \\
\hline$R_{f}$ & Retardation factor due to adsorption and desorption & - \\
\hline$R_{f}^{*}$ & $\begin{array}{l}\text { Retardation factor due to precipitation as well as adsorption and desorp- } \\
\text { tion }\end{array}$ & - \\
\hline$\rho_{b}$ & Fluid density & $\mathrm{kg} \mathrm{m}^{-3}$ \\
\hline$\rho_{s}$ & Density of aquifer solid & $\mathrm{kg} \mathrm{m}^{-3}$ \\
\hline$S$ & Storage coefficient & - \\
\hline$S_{a}$ & Scavenging rate of radionuclide from brine by adsorption to rock surfaces & $\mathrm{Bq} \mathrm{kg}^{-1}$ \\
\hline$S_{p}$ & $\begin{array}{l}\text { Scavenging rate of radionuclide from brine by co-precipitation with min- } \\
\text { erals }\end{array}$ & $\mathrm{Bq} \mathrm{kg}{ }^{-1}$ \\
\hline$S_{f v}$ & $\begin{array}{l}\text { Specific surface area of a fracture, expressed as area of solid per volume } \\
\text { of fluid contacting the solids }\end{array}$ & $\mathrm{m}^{2} \mathrm{~m}^{-3}$ \\
\hline$S_{\text {eff }}$ & Effective surface area of equivalent fracture & $\mathrm{m}^{2}$ \\
\hline$S_{\text {total }}$ & Specific surface area of equivalent fracture & $\mathrm{m}^{2} \mathrm{~m}^{-3}$ \\
\hline$\tau_{p, i}$ & Mean time for nuclide $i$ in solution to precipitate in minerals & s \\
\hline$\tau_{w, i}$ & Mean time for leach nuclide $i$ from solid phases & s \\
\hline$w_{f}$ & Fracture width & $\mathrm{m}$ \\
\hline$v_{f}$ & Advection velocity of fracture fluid & $\mathrm{m} \mathrm{s}^{-1}$ \\
\hline$x$ & Distance & $\mathrm{m}$ \\
\hline$\zeta$ & Variance from fracture width & - \\
\hline ' & Superscript referring to radioactive parent & - \\
\hline
\end{tabular}




\section{List of Figures}

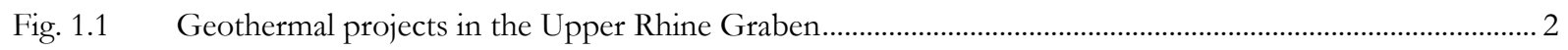



Fig. 1.3 Distribution of the ${ }^{232} \mathrm{Th}$ series radionuclides in geothermal brines .............................................................. 7

Fig. 2.1 Tectonic map of the study area (left) and cross section (right) ................................................................... 15

Fig. 2.2 Scheme of Bruchsal surface installation with the sampling points (P1-P6) .............................................. 17

Fig. 2.3 Sampling point after reconstruction (left) and positioning of sampling tabs within the pipe (right) .........17

Fig. 2.4 Simulation of the time-depended evolution of standardized activity of ${ }^{228} \mathrm{Ra}$ and its daughters...............23

Fig. $3.1 \quad$ Location of the Bruchsal geothermal site in the Upper Rhine Graben.......................................................... 37

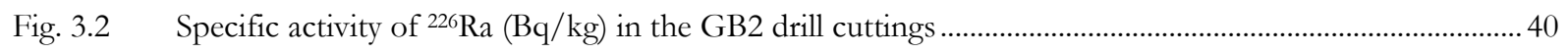

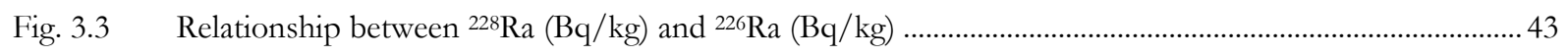

Fig. 3.4 Radionuclide disequilibrium within the reservoir section of GB2 ..............................................................4



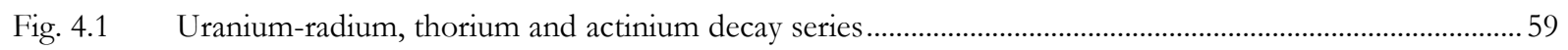

Fig. 4.2 Geological WNW - ESE cross section of the Bruchsal GB2 production well ..........................................62

Fig. 4.3 Several successive transformations within the ${ }^{232} \mathrm{Th}$ decay series for the Bruchsal brine ...........................66

Fig. 4.4 Uranium and thorium series disequilibria in the Bruchsal brine.............................................................. 70

Fig. 4.5 Schematic representation of the water-rock interaction model suggested by Ku et al. (1992) .................. 74

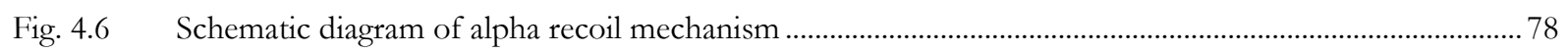

Fig. 4.7 Comparison of MIN3P and CAS model for one dimensional transport of ${ }^{224} \mathrm{Ra}$........................................ 82

Fig. 4.8 Re-calculation of radium co-precipitation with barite from literature ......................................................... 84

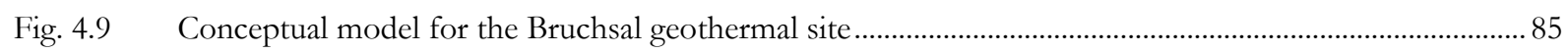



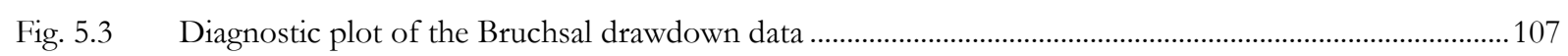

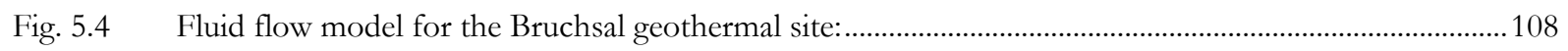

Fig. $5.5 \quad{ }^{222} \mathrm{Rn}$ diffusion length $(L)$ normalized to radon fluid activity .....................................................................114

Fig. 5.6 Interaction between site-specific ${ }^{222} \mathrm{Rn}$ activity in the large fracture fluid $\left(\right.$ atoms s$\left.{ }^{-1} \mathrm{~L}^{-1}\right)$........................115

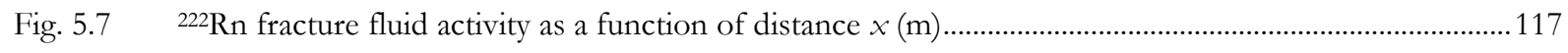

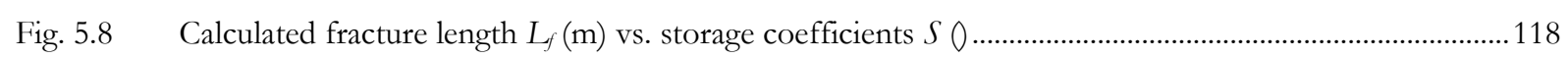

Fig. 5.9 Surface area of the equivalent fracture $\left(S_{f i}\right)$ for varying fracture widths $\left(w_{f}\right)$..........................................119

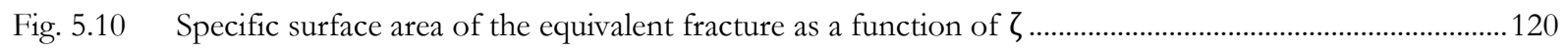




\section{List of Tables}

Table 2.1 Measuring parameters and the related test methods used for the Bruchsal fluid monitoring 18

Table 2.2

Alpha-ray and gamma-ray energies $\mathrm{E}_{\alpha, \gamma}(\mathrm{keV})$ and emission probability $\mathrm{P}_{\alpha}$

Table 2.3 Specific activities of ${ }^{238} \mathrm{U},{ }^{232} \mathrm{Th}$ and ${ }^{235} \mathrm{U}$ decay series radionuclides dissolved in the Bruchsal brine. 26

Table 2.4 Radium activities and activity ratios in brine collected from GB2 well .. 27

Table 2.5 Specific activity of ${ }^{232}$ Th series nuclides measured in the GB2 drill cuttings.

Table 4.1 Overview of the nuclides used for determining Ra activities in the Bruchsal brine. 


\section{Table of Contents}

Abstract $\quad$ i

Kurzfassung iii

Acknowledgements $\quad$ v

List of Symbols $\quad$ vi

List of Figures viii

List of Tables $\quad$ ix

Table of Contents $\quad x$

1 Introduction 1

1.1 Motivation 1

1.2 Methodological background and approaches 5

1.2.1 Decay-series disequilibria in geothermal systems 5

1.2.2 Behavior of radium in $\mathrm{NaCl}$ brines 6

1.2.3 Modelling the disequilibria in water-rock systems $\quad 7$

1.2.4 Application field for geothermal exploitation 8

1.3 Research approach 8

1.4 Thesis outline $\quad 10$

2 Design and setup of a radiochemical monitoring system 14

$\begin{array}{lll}2.1 & \text { Introduction } & 14\end{array}$

2.2 Study area and test site 14

2.2.1 Upper Rhine Graben 14

$\begin{array}{lll}\text { 2.2.2 Bruchsal site } & 15\end{array}$

2.3 Bruchsal radiochemical monitoring system 16

$\begin{array}{lll}\text { 2.3.1 Setup of the sampling system } & 16\end{array}$

$\begin{array}{lll}\text { 2.3.2 Parameter selection } & 18\end{array}$

2.4 Supportive analysis and data acquisition 20

2.5 Spetrometry surveys of Th-U series nuclides 21

2.5.1 Gamma spectrometry 21

2.5.2 Alpha spectrometry 24

2.5.3 Uncertainties 25

2.6 Selected results 25

3 Identification of fractured zones in geothermal reservoirs in sedimentary basins: A radionuclide-

based approach $\quad 33$

3.1 Introduction $\quad 35$

3.2 Geological setting 36

3.3 Material and method 38

3.4 Results 41

$\begin{array}{lll}\text { 3.4.1 Alteration petrography } & 41\end{array}$

3.4.2 Results from spectrometric measurements $\quad 42$

3.4.3 Signature of radium isotopes $\quad 42$

3.4.4 Uranium series disequilibrium 43

3.4.5 Thorium decay series radionuclides 44

$\begin{array}{lll}3.5 & \text { Discussion } & 45\end{array}$ 
$\begin{array}{llr}3.6 & \text { Conclusions } & 52\end{array}$

$4 \quad$ Water-rock interactions in the Bruchsal geothermal system by U-Th series radionuclides $\quad 57$

$\begin{array}{llr}4.1 & \text { Introduction } & 59\end{array}$

$\begin{array}{llr}4.2 & \text { Geothermal context } & 61\end{array}$

4.2.1 Upper Rhine Graben (URG) $\quad 61$

$\begin{array}{ll}\text { 4.2.2 Bruchsal site } & 61\end{array}$

$\begin{array}{lll}4.3 & \text { Methodology } & 63\end{array}$

4.3.1 Sampling and analytical methods $\quad 63$

4.3.2 Calculation of Ra activities from gamma spectrometry $\quad 64$

$\begin{array}{llr}4.4 & \text { Results } & 67\end{array}$

$\begin{array}{lll}\text { 4.4.1 Major and trace elements } & 67\end{array}$

$\begin{array}{lll}\text { 4.4.2 U-Th decay series radionuclides } & 68\end{array}$

$\begin{array}{ll}\text { 4.4.3 Radium isotopes } & 71\end{array}$

4.5 Modelling the disequilibria in water-rock systems 73

4.5.1 Model assumptions suggested by Ku et al. (1992) 73

$\begin{array}{lll}\text { 4.5.2 Governing equations } & 76\end{array}$

$\begin{array}{lrr}4.6 & \text { Model performance } & 80\end{array}$

$\begin{array}{llr}\text { 4.6.1 Recoil mechanism } & 81\end{array}$

$\begin{array}{ll}\text { 4.6.2 Kinetic approach for solid solutions } & 81\end{array}$

$\begin{array}{lll}4.7 & \text { Discussion } & 84\end{array}$

4.7.1 Alpha recoil supply rates and recoil efficiencies 86

$\begin{array}{ll}\text { 4.7.2 Diffusional flux } & 87\end{array}$

$\begin{array}{lll}\text { 4.7.3 Ra removal by adsorption and precipitation } & 89\end{array}$

$\begin{array}{ll}\text { 4.7.4 Ra supply by groundwater flow } & 91\end{array}$

$\begin{array}{llr}\text { 4.7.5 Steady state Ra fluid activities } & 92\end{array}$

$\begin{array}{ll}4.8 \text { Conclusions } & 93\end{array}$

5 Integrated approach into the characterization of the fracture network of a geothermal reservoir 100

$\begin{array}{llr}5.1 & \text { Introduction } & 102\end{array}$

$\begin{array}{llr}5.2 & \text { Geothermal context } & 103\end{array}$

$\begin{array}{lll}\text { 5.2.1 Upper Rhine Graben } & 103\end{array}$

$\begin{array}{ll}\text { 5.2.2 Bruchsal site } & 104\end{array}$

$\begin{array}{ll}\text { 5.2.3 Well testing and reservoir parameter } & 106\end{array}$

$\begin{array}{llr}5.3 & \text { Conceptual model } & 107\end{array}$

$\begin{array}{ll}5.4 \text { Governing equation } & 110\end{array}$



$\begin{array}{llr}5.5 & \text { Results and discussion } & 113\end{array}$

5.5.1 Estimating in situ fracture width from ${ }^{222} \mathrm{Rn}$ flux $\left(w_{f}\right) \quad 113$

$\begin{array}{ll}\text { 5.5.2 Estimating site-specific fracture length }\left(L_{f}\right) & 115\end{array}$

$\begin{array}{ll}\text { 5.5.3 Estimating fracture surface area }\left(S_{f i}\right) & 118\end{array}$

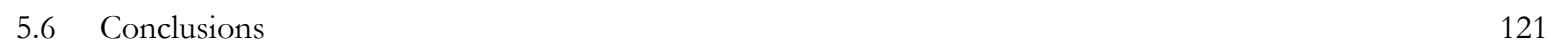

6 Conclusions $\quad 126$

6.1 Integration and evaluation of methods of investigation and results 126

6.1.1 Detecting productive geothermal horizons while reservoir engineering 126

6.1.2 Assessing Th-U migration behavior during power plant operation 127

$\begin{array}{ll}\text { 6.1.3 Up-scaling of geothermal power plants } & 128\end{array}$ 


\section{Chapter I}

\section{Introduction}

\subsection{Motivation}

Geothermal energy is a renewable energy resource offering both, power production as well as direct heat supply on a temperature level sufficient for industrial processes and district heating with a remarkably high system availability. Its contribution to the world's energy consumption amounted to 630 petajoules in 2018, equally shared by electricity and heat (REN21, 2019).

Germany uses its low to medium enthalpy geothermal resources for geothermal energy exploitation. Thus, an increasing number of geothermal power and heat plants came into operation during the last two decades summing up to 37 installations in recent years. Following the recent trend to decarbonize Germany's energy sector, it is expected that large district heating systems will experience a fuel switch from fossil combustibles to geothermal heat in future (Weimann, 2019).

The three most promising geothermal provinces in Germany are the Upper Rhine Graben (URG), the Bavarian Molasse Basin and North German Basin. It is remarkable that these provinces cover seven of twelve German metropolitan regions where large district heating systems supplied by fossil fired plants exist. This coexistence of resource and consumers offers the opportunity to supply heat from deep geothermal reservoirs in the range of 30 to 50 TWh per year (Weinmann, 2019).

The Upper Rhine Graben with two large district heating systems in Mannheim and Karlsruhe may serve as an example. A total of six geothermal plants is under operation in this region, three of them producing electricity while the remaining plants are heat stations or, in the case of Bruchsal geothermal installation, providing combined heat and power (Vidal and Genter, 2018) (Fig. 1.1).

Besides appropriate surface installations and the deep geothermal wells, a sufficient reservoir performance governs the geothermal energy production from deep resources (e.g. Grant and Bixley, 2011). As the primary permeability of geothermal reservoirs is linked to rock fractures, the reservoir performance strongly depends on the nature of the fracture network.

Thus, major parts of fluid flow and transport processes are limited to a relatively small percentage 
of the total reservoir volume. The determination of these parameters is part of the reservoir engineering which is one of the governing disciplines for developing a site specific geothermal conceptual model (Grant and Bixley, 2011). Conceptual models can be implemented already during the exploration phase of a geothermal project. It is based on the joint interpretation of all relevant data sets deriving from different sources, e.g. geophysics, geochemistry or hydrogeology, and allows the operators to optimize all work packages starting from exploration of the resource up to reservoir enhancement and even during plant operation.

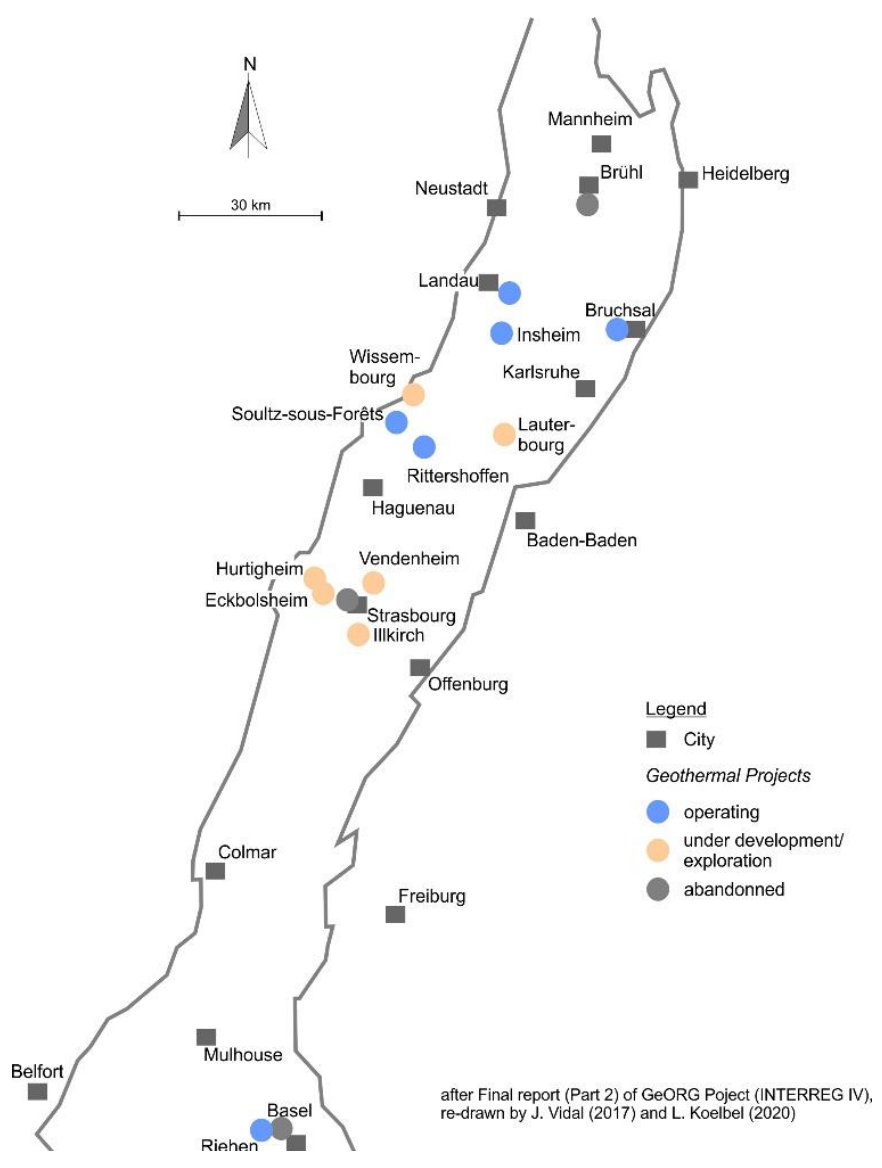

Fig. 1.1: Geothermal projects in the Upper Rhine Graben.

With just a few exceptions, geothermal plants in Central Europe consist of just one production and injection well (well doublet), while in a global perspective geothermal sites are characterized by a certain number of wells. A multi well setup offers several advantages compared to a well doublet which are not only restricted to a higher energy output, but also to an increase of plant availability, optimized logistics and other quantity effect related improvements (e.g. Kölbel, 2015). Tapping the geothermal resource by a certain number of wells needs a sophisticated conceptual model based on a proper understanding of the reservoir conditions and parameters. 

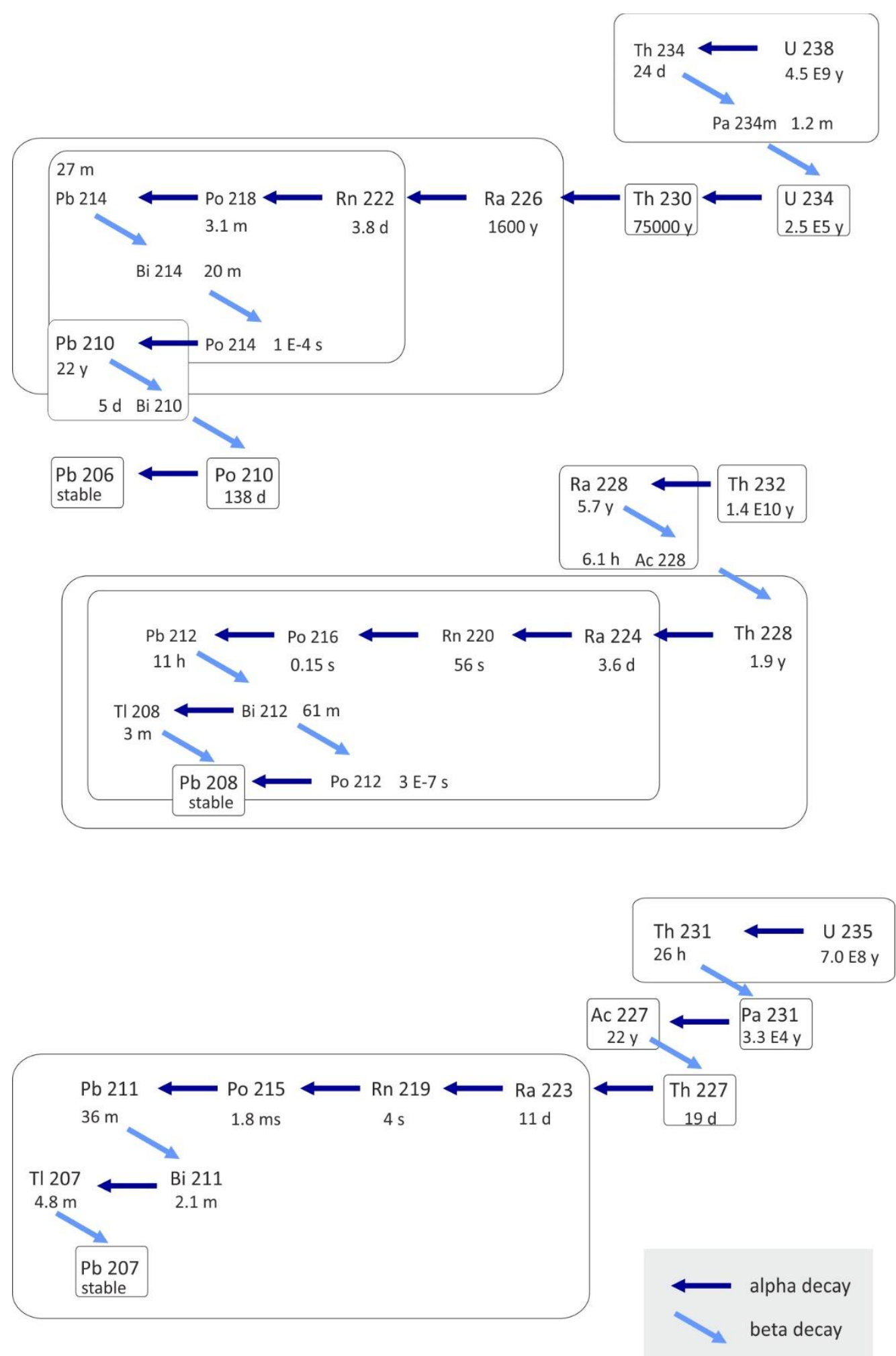

Fig. 1.2: ${ }^{238} \mathrm{U},{ }^{232} \mathrm{Th}$ and ${ }^{235} \mathrm{U}$ decay series. For radionuclides with more than one decay mode, only the most frequently occurring is given. Redrawn after Surbeck, 1995. 
A differentiated analysis of naturally occurring radionuclides of the ${ }^{238} \mathrm{U},{ }^{232} \mathrm{Th}$ and ${ }^{235} \mathrm{U}$ decay series (Fig. 1.2) might provide an additional tool to support reservoir engineering and thus, improve the conceptual model during various stages of geothermal project development. The natural decay series comprise radioactive elements with different physico-chemical properties. They are linked through radioactive decay and growth. Especially fluid phases are quite sensitive to radioactive exchange. Their isotopic signatures are strongly governed by physico-chemical interactions with the associated solids. Since there is more than one isotope with different half-lives for a given element, it allows the determination of water-rock interaction rates across different timescales $(\mathrm{Ku}$ et al., 1992). The different geochemical behaviors of these radionuclides result in radioactive disequilibria not only in geothermal brines, but also in the adjoining reservoir rocks.

The detection of pay zones is one of the major challenges in geothermal reservoir engineering. Their precise determination already while drilling has a cost-effective impact at least on drilling depth, casing design and the well testing scheme. Various scientific disciplines as well as a certain range of different techniques, often based on geophysical well logging tools, are involved to support this issue (Serra 1984; Ellis and Singer, 2007). An appropriate analysis of the radionuclide signatures of cutting sampled while drilling is a suitable approach to gather additional information from certain depth intervals. Considering a lag time correction, they might provide a precise data set from a certain depth without extra runs of logging tools.

Modelling of radionuclide disequilibria can provide information about the respective water-rock interaction processes controlling radionuclide supply into and scavenging from solution, respectively (e.g. Lou et al., 2000; Tricca et al., 2000). This information is very useful to investigate the long-term migratory behavior of uranium and thorium series radionuclides which is not only an important issue for nuclear waste disposal, but also for geothermal sites in terms of handling radionuclide-bearing mineral precipitations in surface installations. The latter results from variations of fluid temperature and pressure along the circulation path of the geothermal brine through the underground access wells and in parts of the surface plant. This information enables plant operators to the refinement of the health and safety standards on site and support also a competent waste management (Eggeling et al., 2013).

Furthermore, understanding the migratory behavior of radionuclides in the reservoir might be useful for the sustainable management of the geothermal reservoir. Since fracture zones provide the major conduits for hydraulic flow, information about site-specific fracture geometry is of great importance. To solve this issue, naturally occurring radionuclides might employ, under certain conditions, as natural radiotracers. One example is ${ }^{222} \mathrm{Rn}$ which has been extensively used as a tracer for the study of groundwater (e.g. Tanner, 1964; Lawrence et al., 1991) and geothermal systems (e.g. Andrews et al., 1986; Folger et al., 1997) in the past. Estimates of the flow regimes are generally based on hydrogeological data such as well test data. 
Using an integrated approach into the characterization of the fracture network of a geothermal reservoir might be an option to reduce interpretative hydraulic parameter ambiguity. After integration of the resulting data set in the conceptual model, the operators of a geothermal plant may use this information for scaling up their power or heat output by additional wells.

\subsection{Methodological background and approaches}

\subsubsection{Decay-series disequilibria in geothermal systems}

Naturally occurring radionuclides are widespread in natural systems. Primordial nuclides such as ${ }^{238} \mathrm{U},{ }^{232} \mathrm{Th}$ and ${ }^{235} \mathrm{U}$ are notable for their long half-lives (similar to the age of the earth) and are still present in measurable quantities today. They form the starting points of the uranium, thorium and actinium series and decay through a sequence of short-lived intermediate daughter nuclides to stable isotopes of lead (Fig. 1.2).

In the natural decay series, the daughters evolve into a secular radioactive equilibrium with their ${ }^{238} \mathrm{U}$, ${ }^{232} \mathrm{Th}$ and ${ }^{235} \mathrm{U}$ parents and the parent/daughter activity (disintegration) ratio becomes unity. This type of radioactive equilibrium typically occurs when the half-life of the parent is by far larger compared to the daughter's half-life ( $\left.\mathrm{t}_{1 / 2}(\mathrm{~A})>>\mathrm{t}_{1 / 2}(\mathrm{~B})\right)$. The time necessary to reach radioactive equilibrium depends on the daughter's half-life, but also on the parent/daughter ratio of half-lives.

In closed geological systems, more than $99 \%$ of the radioactive equilibrium will be reached after seven half-lives of the longest-lived daughter nuclides in the series. These are ${ }^{234} \mathrm{U}\left(\mathrm{t}_{1 / 2}=2.5 \times 10^{5}\right.$ y), ${ }^{228} \mathrm{Ra}\left(\mathrm{t}_{1 / 2}=5.7 \mathrm{y}\right)$ and ${ }^{231} \mathrm{~Pa}\left(\mathrm{t}_{1 / 2}=3.3 \times 10^{4} \mathrm{y}\right)$ in the uranium, thorium, and actinium series, respectively (Lieser, 2001). Consequently, an equilibrium is usually found in natural systems that are older than $2 \times 10^{6}$ years. Such systems are, for example, old crystalline rocks or old sediments with significantly low porosity and low permeability.

In geothermal energy systems, however, a huge quantity of geothermal fluid is circulated in the underground reservoir and interacts with the host rock during exploitation phase. During the operation of a geothermal power plant, hot water and/or steam from the underground is transferred to the surface via a production well. After delivering its usable thermal energy to the power plant, the cooled fluid will be pumped back into the reservoir zone via injection wells. As a result of cooling the geothermal fluids for power and heat production, and the associated pressure drop, mineral precipitations within the geothermal surface installations might be observed, some of them incorporating radionuclides.

Thus, geothermal systems are not closed systems because of the physico-chemical reactions between the hot geothermal fluid and the host rock. In systems where an exchange of radionuclides can take place between different phases, a fractionation of parent and daughter nuclides within the 
same decay chain can be observed due to their differing chemical and physical properties (Hussain and Krishnaswami, 1980). This fractionation results in a state of radioactive disequilibrium within the phases and is typical in multi-phase-systems such as aquifers, where liquid, solid and gaseous phases often coexist.

\subsubsection{Behavior of radium in $\mathrm{NaCl}$ brines}

Radioactive disequilibria are frequently observed in $\mathrm{NaCl}$-dominated geothermal brines enriched with $\mathrm{CO}_{2}$ (e.g. Zukin et al., 1987, Condomines et al., 2012, Eggeling et al., 2013). Here, radium isotopes $\left({ }^{228} \mathrm{Ra},{ }^{226} \mathrm{Ra},{ }^{224} \mathrm{Ra},{ }^{223} \mathrm{Ra}\right)$ have activities by far exceeding those of their thorium progenitors. Thus, the supply of radium to the geothermal brine is primarily by the interaction with the reservoir rock than by the decay of thorium in solution.

The low activity of thorium observed in these geothermal fluids is mainly due to its poor solubility. Thorium is generally considered as a very insoluble element in aquatic environments. Since thorium occurs only in 4+ oxidation state, these tetravalent ions are readily hydrolyzed to form insoluble hydroxide complexes. The type and stability range of the individual species is dependent on Eh and $\mathrm{pH}$ value, temperature, and pressure conditions, but also on the concentration of anions (Osmond and Cowart, 1992).

The behavior of radium in geothermal brines is largely affected by salinity, temperature, and redox state. Previous studies have shown that radium concentrations are often high in saline aqueous solutions (Kraemer and Reid, 1984; Dickson, 1985) and geothermal brines (Hammond et al., 1988; Rihs and Condomines, 2002), but rather low in low-temperature, low-salinity groundwaters (Krishnaswami et al., 1982; Luo et al., 2000; Porcelli et al., 2014). Zukin et al. (1987) showed that chloride complexes increase the radium mobility significantly. An increase of salinity and ionic strength can also produce a displacement of radium into solution because of the competition with other cations for ion exchange sites (Langmuir and Melchior, 1985). High temperatures also tend to increase radium activity in brine. Ames et al. (1983) presented that with increasing temperatures the activity of dissolved radium also increases by reducing adsorption coefficients for the divalent $\mathrm{Ra}^{+2}$ ion. $\mathrm{A}$ reducing environment as it is typically given for deep geothermal reservoirs may encourage the release of radium into solution. Kadko (1980) found that the radium activity is high in low Eh conditions to the absence of $\mathrm{MnO}_{2}$ on aquifer solids which can strongly adsorb radium.

Fig. 1.3 displays a scheme of the distribution of ${ }^{232} \mathrm{Th}$ series radionuclides according to their geochemical behaviors as typically found in deep geothermal brines. Isotopes with low solubility are rapid adsorbed from solution creating significant radioactive disequilibria; not only in fluid-phase, but also, in the associated host rocks. The supply of isotopes to solution is governed by physical and chemical processes such as alpha recoil, desorption, or dissolution. 


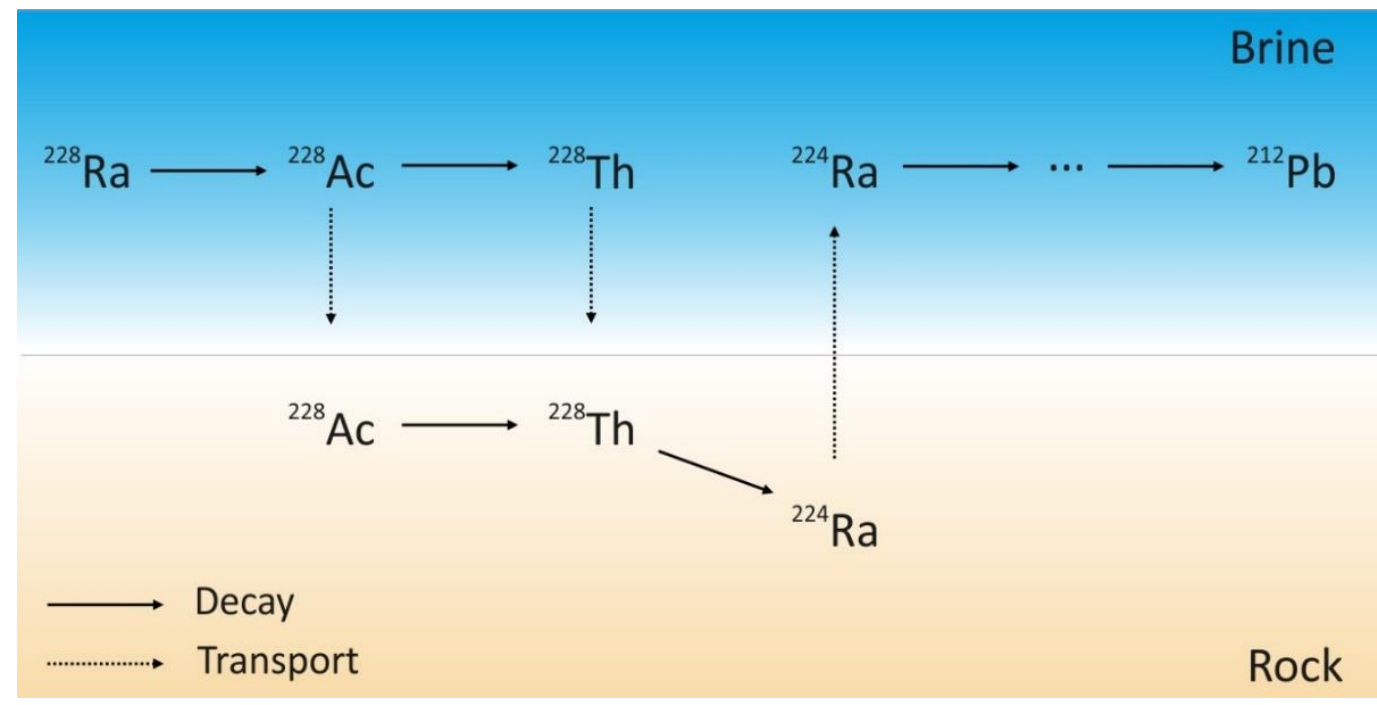

Fig. 1.3: Distribution of the ${ }^{232} \mathrm{Th}$ series radionuclides in geothermal brines. Rapid exchange of isotopes between the geothermal brine and rock results in radioactive disequilibria.

\subsubsection{Modelling the disequilibria in water-rock systems}

The change of activity of a particular radioactive element dissolved in geothermal brine with time is dependent upon several interaction processes. Tricca et al. (2001) describe these processes as physico-chemical reactions taking place between three groups: the aqueous phase, the mineral grains and a reactive surface layer with a certain specific area and thickness. The transfer rate of a radionuclide from the rock material into the solution depends on: (a) the in-situ radioactive decay of its dissolved parent, (b) the desorption from the surface coating, (c) the alpha-recoil across the solid-liquid interface within a distance of several tens of nanometers, and (d) the dissolution of the host rock. The removal of a radionuclide from the fluid depends on: (a) its radioactive decay in solution, (b) the adsorption onto the surface layer, and (c) the incorporation into precipitates $(\mathrm{Ku}$ et al., 1992).

Several mathematical solutions of simplified aquifer models have been widely studied in the past. Andrews et al. $(1982,1989)$ considered physico-chemical mechanisms for radionuclide supply and removal and calculated the timescale of water-rock interactions. However, the authors did not consider transport by advection. Krishnaswami et al. (1982) computed rate constants of sorption processes. Furthermore, they determined residence times of daughter nuclides by means of alpharecoil input from ${ }^{222} \mathrm{Rn}$ activities, although without considering the effects of advective transport and mineral dissolution/precipitation. Davidson and Dickson (1986) suggested a model of uranium and radium isotopes transport including dispersive flow, but without considering precipitation and dissolution processes. Ku et al. (1992) proposed a model that accounts for radionuclide transport by advection and first-order kinetics, sorption-desorption, and dissolution-precipitation of U-Th 
series radionuclides processes as well as the supply from alpha recoil. Tricca et al. (2000, 2001) suggested a model for the combined groundwater transport of naturally occurring $U$, Th, Ra, and $\mathrm{Rn}$ isotopes with regards to advective transport as well as the physico-chemical processes of weathering, decay, alpha-recoil and sorption at the water-rock interface.

\subsubsection{Application field for geothermal exploitation}

Studying the Th-U series disequilibria provides several advantages for geothermal energy exploitation.

First, the investigation of radionuclide signatures in rocks can have a practical benefit for geothermal reservoir engineering. In the early project stage, drill cuttings are of interest since they can provide relevant information about reservoir characteristics. The drill cuttings can be analyzed and interpreted with some acceptable time delay during drilling operation is still in progress. Radiochemical surveys can provide relevant information about the mobilization and distribution of natural radionuclides. Here, special attention must be paid to fracture zones which represent a substantial portion of permeability. Since they channel the geothermal fluid in the reservoir section, fractured zones might be indicated by a fractionation of parent and daughter nuclides in the solids resulting from intensive water-rock interaction.

Second, Th-U series disequilibria in geothermal fluids can provide site-specific information about radionuclide migration in the reservoir section. Since the geothermal brine is overly sensitive to radionuclide exchanges, it displays the extent of radioactive disequilibrium very clear. Regular monitoring of the radionuclide signatures in brine allows to learn about the geochemical behavior of natural radionuclides within the flow system. A joint evaluation about radionuclide signatures in rock and brine provides a way to access the mobilization of Th-U series nuclides due to physicochemical reactions.

Third, understanding the behavior of natural radionuclides in the geothermal system may enable the plant operators to assess the consequences to the power plant operation. Th-U series nuclides can be transported into the geothermal power plant and incorporate in mineral precipitations. Sitespecific information dealing with the formation of radionuclide-bearing scalings and their growth characteristics can be used to determine strategies to avoid such precipitations. In addition, the operator meets his obligation concerning the fulfilment of health and safety standards on site combined with an appropriate waste management.

\subsection{Research approach}

The primary objective of the present thesis to provide additional options to enhance conceptual 
models of geothermal sites. Under this premise the development of a site-specific reservoir characterization by means of Th-U series nuclides is an essential step. Analyzing isotopic signatures of the Bruchsal reservoir rock material is conducted aiming at the identification of permeable zones of active fluid circulation in close vicinity of boreholes. The detection of productive geothermal reservoir sections (pay zones) is one of the major challenges in geothermal exploration and involves the application of various scientific disciplines such as geology, geochemistry as well as a range of geophysical well logging techniques including among others geoelectrics, gravity, magnetics, and seismics (Ellis and Singer, 2007; Grant and Blixley, 2011; Ochieng, 2013).

These fracture zones provide a substantial contribution to the effective hydraulic conductivity and pool the geothermal fluid circulation. In this context, water-rock interaction processes and the resulting changes in the isotopic composition of fluid and rock provide information on the type and the degree of fluid flow in the area of the fractured material. Consequently, the understanding about the interaction between the hot geothermal brine with the adjoining reservoir rock is crucial for a successful reservoir engineering.

In the past, much focus has been placed by several scientists on the uranium-thorium series disequilibrium in geothermal fluids because it provides site-specific information about radionuclide migration in the reservoir section (e.g. Zukin et al., 1987; Hammond et al., 1988; Lou et al., 2000, Tricca et al. 2000). However, since the modelling of radioactive disequilibria requires both types of information, the composition of the fluid and the solid phase, the Bruchsal reservoir rock needs to be intensively examined based on cuttings from the existing geothermal boreholes.

This thesis is intended to illustrate how naturally occurring radionuclides can support geothermal energy exploitation in the Upper Rhine Graben during various stages of a project's life cycle. The methodological approach is based on the detection of decay-series disequilibria within the geothermal system. These radioactive disequilibria are caused by a relatively rapid exchange of isotopes between brine and rock resulting in a fractionation of daughter and parent nuclides. Site-specific drill cuttings and water samples are investigated by spectrometric measurement methods. Isotopic fractionation processes observed in the rock material may indicate permeable zones within the reservoir section characterized by water-rock interactions. Th- $U$ series disequilibria of the geothermal brine is used to learn about the behavior of radionuclides within the flow system and the timescale of water-rock interactions. These are useful information for the operator since natural radionuclides affect the power plant operation. For example, radionuclide-bearing mineral precipitations within the surface installation system such as pipelines may occur because of alternating temperature and pressure during the passage of the produced fluid through the geothermal plant.

The mathematically treatment is based on $\mathrm{Ku}$ et al. (1992) whose water-rock interaction model allows the detailed analysis of physico-chemical reactions as well as advective transport phenomena. The modelling part of this thesis focusses on the behavior of radium isotopes ${ }^{228} \mathrm{Ra},{ }^{226} \mathrm{Ra}$, 
${ }^{224} \mathrm{Ra},{ }^{223} \mathrm{Ra}$ ) since they are highly soluble in the Bruchsal brine and their range in half-lives and their interrelation in the respective decay chain allows the determination of water-rock interaction rates across different timescales.

Special emphasis is placed on the model adjustment to a fracture-dominated radionuclide transport as determined for the Bruchsal geothermal site from well test analysis since the model assumption suggested by Ku et al. (1992) focuses on one-dimensional flow through porous media. Th-U series radionuclides dissolved in the Bruchsal geothermal brine, especially the noble gas ${ }^{222} \mathrm{Rn}$, might be a part of the characterization of the site-specific fracture network. Since radon migrates within the rock mass by diffusion and/or advection through pores, macro-pores and fractures (e.g. Nazaroff and Nero, 1988; Ferry et al., 2002), a joint inversion of a radon diffusion model including pumping test is developed to assess site-specific fracture dimensions.

\subsection{Thesis outline}

The thesis has a cumulative structure and is divided into the following chapters:

Chapter 2 describes the local geological setting as well as the design, setup and implementation of a radiochemical monitoring system. Long-term fluid sampling generates a comprehensive data set. Analysis of borehole cutting samples complete these data and provide additional information about the geothermal reservoir section.

Chapter 3 presents a new approach for the identification of fractured zones of active, or at least previously active, fluid circulation in boreholes based on activity ratios of thorium-uranium series radionuclides in rocks. This method has been tested at the Bruchsal geothermal site in Germany.

Chapter 4 refers to the study of water-rock interaction processes using U-Th decay-series disequilibria. The modelling part of this study focuses on radium isotopes acquiring information about the rates of water-rock interactions and hydrogeological properties of geothermal systems.

Chapter 5 addresses an integrated approach on site-specific fracture dimensions. A mass balance approach of ${ }^{222} \mathrm{Rn}$ is used to gain information about site-specific fracture geometry such as fracture length, width, and surface.

Chapter 6 discusses the possibilities and limits of the research findings represented within this thesis and provides an outlook for further research.

Please note that the original published versions are slightly modified to receive a consecutive numbering of tables and figures within this work. Furthermore, a separate reference list is attached to the end of each chapter. 


\section{References}

Ames, L.L., McGarrah, J.E., Walker, A.B., Salter, P.F., 1983. Uranium and radium sorption on amorphous ferric oxyhydoxide. Geochemical Geology 40, 135-148.

Andrews, J. N., Giles, I. S., Kay, R. L. F., Lee, D. J., Osmond, J. K., Cowart, J. B., Fritz, P., Barker, J. F., Gale, J., 1982. Radioelements, radiogenic helium and age relationships for groundwaters from the granites at Stripa, Sweden. Geochimica et Cosmochimica Acta, 46, 1533-1543.

Andrews J.N., Hussain N., Batchelor A.S., Kwakwa K., 1986. ${ }^{222}$ Rn solution by the circulating fluids in a 'hot dry rock' geothermal reservoir. Appl. Geochim. 1, 647-657.

Condomines, M., Gourdin, E., Gataniou, D., Seidel, J.-L., 2012. Geochemical behaviour of Radium isotopes and Radon in a coastal thermal system (Balaruc-les-Bains, South of France). Geochimica et Cosmochimica Acta 98, 160-176.

Davidson, M. R., Dickson, B. L., 1986. A porous flow model for steady-state transport of radium in ground waters. Water Resources Research, 22, 34-44.

Dickson, B.L., 1985. Radium isotopes in saline seepage, southwestern Yilgarn, Western Australia. Geochim. Cosmochim. Acta 49, 349-360.

Eggeling, L., Genter, A., Kölbel, T., Münch, W., 2013. Impact of natural radionuclides on geothermal exploitation in the Upper Rhine Graben. Geothermics 47, 80-88.

Ellis, D.V., Singer, J.M., 2007. Well Logging for Earth Scientists, Springer, Dordrecht.

Ferry C., Richon P., Beneito A., Cabrera J., Sabroux J.C, 2002. An experimental method for measuring the radon-222 emanation factor in rocks. Radiation Measurement, 35, 579-583.

Folger, P., Poeter, E., Wanty, R.B., Day, W., Frishman, D., 1997. ${ }^{222}$ Rn transport in a fractured crystalline rock aquifer: results from numerical simulations. Journal of Hydrology 195, 45-77.

Grant, M.A., Bixley, P.F., 2011. Geothermal Reservoir Engineering, Elsevier, Oxford.

Hammond, D.E., Zukin, J.G., Ku, T.L., 1988. The kinetics of radioisotope exchange between brine and rock in a geothermal system. J. Geophys. Res. 93 (13), 175-186.

Hussain, N., Krishnaswami, S., 1980. U-238 series radioactive disequilibrium in groundwater: implications to the origin of U-234 excess and fate of reactive pollutants. Geochimica et Cosmochimica Acta 44, 287-1291.

Kadko, D., 1980. Th-230, Ra-226, and Rn-222 in abyssal sediments. Earth Planet. Sci. Lett. 49, 360-380

Kölbel, T., 2015. Geothermal power 2020: Outlook from a utility. In: Proceedings of the 9 $9^{\text {th }}$ GeoTHERM, Offeburg, 2015.

Kraemer, T.F., Reid, D.F., 1984. The occurrence and behavior of radium in saline formation water of the U.S. Gulf 
Coast region. Isotope Geoscience 2, 153- 174.

Krishnaswami, S., Graustein, W.C., Turekian, K.K., Dowd, J.F., 1982. Radium, thorium and radioactive lead isotopes in groundwaters: Applications to the in-situ determination of adsorption-desorption rate constants and retardation factors. Water Resour. Res. 18 (6), 1633-1675.

Ku, T.-L., Luo, S., Leslie, B.W., Hammond, D.E., 1992. Decay-series disequilibria applied to the study of water-rock interaction and geothermal systems, in: Ivanovich, M., Harmon, R.S. (Eds.), Uranium-series Disequilibrium: Applications to Earth, Marine, and Environmental Sciences. Clarendon Press, Oxford, 631-668.

Langmuir, D., Melchior, D., 1985. The geochemistry of Ca, Sr, Ba, and Ra sulfates in some deep brines from the Palo Duro Basin, Texas. Geochim. Cosmochim. Acta 49, 2423-2432.

Lawrence, E.P., Poeter, E., Wanty, R., 1991. Geohydrologic, geochemical, and geologic controls on the occurrence of radon in groundwater near Conifer, Colorado, USA. Journal of Hydrology 127, 367-386.

Lieser, K., 2001. Nuclear and Radiochemistry. Fundamentals and Applications (2 Ausg.). Weinheim: Wiley-VCH.

Luo, S., Ku, T.-L., Roback, R., Murrell, M., McLing, T.L., 2000. In-situ radionuclide transport and preferential groundwater flows at INEEL (Idaho): Decay-series disequilibrium studies. Geochim. Cosmochim. Acta, 64, 867881.

Nazaroff W.W., Nero A.V., 1988. Radon and its decay products in indoor air. United States.

Ochieng, L., 2013. Overview of geothermal surface exploration methods. Short Course VIII on Exploration for Geothermal Resources, Kenya, Oct. 31 - Nov. 22, 2013.

Osmond, J.K., Cowart, J.B., 1992. Ground water. In: Ivanovich, M., Harmon, R.S. (Eds.), Uranium-series Disequilibrium: Applications to Earth, Marine, and Environmental Sciences. Oxford Science Publications, pp. 290.

Porcelli, D., Kim, C.-K., Martin, P., Moore, W.S., Phaneuf, M., 2014. Properties of radium, in: IAEA (Ed.), the environmental behavior of radium (revised edition), technical report series no. 476, Vienna, 6-32.

REN21, 2019. Renewables 2019 Global Status Report (Paris: REN21 Secretariat). ISBN 978-3-9818911-7-1.

Rihs S. and Condomines M., 2002. An improved method for Ra isotope $\left({ }^{226} \mathrm{Ra},{ }^{228} \mathrm{Ra},{ }^{224} \mathrm{Ra}\right)$ measurements by gamma spectrometry in natural waters: applications to $\mathrm{CO}_{2}$-rich thermal waters from the French Massif Central. Chem. Geol. 182, 409-421.

Serra, O., 1984. Fundamentals of well-log interpretation: The acquisition of logging data. Elsevier, Amsterdam.

Surbeck, H., 1995. Determination of natural radionuclids in drinking water; a tentative protocol. The Science of the total Environment 173/174, 91-99.

Tanner, A.B., 1964. Radon migration in the ground: a review, in: Adams, J.A.S and Lowder, W.M. (Eds.), the natural radiation environment, Univ. Chicago Press, Chicago, 161-190.

Tricca, A., D. Porcelli, and G. J. Wasserburg., 2000. Factors controlling the ground water transport of U, Th, Ra, and 
Rn. Proceedings of the Indian Academy of Sciences, 109, 95-108.

Tricca, A., Wasserburg, G.J., Porcelli, D., Baskaran, M., 2001. The transport of U- and Th-series nuclides in a sandy unconfined aquifer. Geochim. Cosmochim. Acta 65, 1187-1210.

Vidal, J., Genter, A., 2018. Overview of naturally permeable fractured reservoirs in the central and southern Upper Rhine Graben: Insights from geothermal wells. Geothermics 74, 57-73.

Weimann, T., 2019: Vorbereitung und Begleitung bei der Erstellung eines Erfahrungsberichts gemäß $\int 97$ Erneuerbare-Energien-Gesetz - Teilvorhaben IIb (Geothermie). Wissenschaftlicher Endbericht.

Zukin, J.G., Hammond, D.E., Ku, T.-L., Elders, W.A., 1987. Uranium-Thorium series radionuclides in brines and reservoir rocks from two deep geothermal boreholes in the Salton Sea geothermal field, southeastern California. Geochim. Cosmochim. Acta 51, 2719-2731. 


\section{Chapter II}

\section{Design and setup of a radiochemical monitoring system}

\subsection{Introduction}

A monitoring system is a general term for the systematic recording of a process by means of technical devices. It includes the data collection and documentation as well as the data evaluation and is characterized by regular repetition. The key objective of a monitoring system is to generate all relevant data sets for analysis and evaluation, which are indispensable to secure a safe and smooth operation.

The aim of monitoring systems in geothermal business concerns environmental and operational subjects on the one hand. On the other hand, scientific issues such as the determination of hydraulic parameters of fractured reservoirs supplement this process. In this thesis, fluid observations are an essential subset of the radiochemical monitoring system. The major target is to gain information not only about the deep geothermal brine, but also about interaction with the reservoir solids with whom it come into contact.

\subsection{Study area and test site}

\subsubsection{Upper Rhine Graben}

The Upper Rhine Graben is part of the European Cenozoic Rift System that extends from the Mediterranean to the North Sea coast (Ziegler, 1992). It is characterized by a NNE-SSW striking extension structure with a length of around $300 \mathrm{~km}$ and a width of up to $40 \mathrm{~km}$. Hercynian basement rocks consist of Gneisses and Paleozoic granites which are covered by sediments of Permian to Cenozoic age. The sediment base in the center of the Upper Rhine valley is located about 3000 $\mathrm{m}$ deeper than at the graben shoulders (Ziegler, 1992). The Upper Rhine Graben offers favorable conditions for the exploitation of geothermal energy because of spatially elevated heat flow and 
thermal anomalies, which are related to large-scale fluid circulation (Pribnow and Schellschmidt, 2000).

The chemistry of fluids in deep aquifers of geothermal provinces in Central Europe varies significantly from site to site. While the thermal water from the Molasse Basin contains a relatively low salt and gas content, the brines from the Upper Rhine Graben and the Northern German Basin are characterized by relatively high salinity and significant gas content (Wolfgramm and Seibt, 2008). Even in the same province the fluid properties may differ from site to site (Aquilina et al., 1997; Schneider et al., 2014).

\subsubsection{Bruchsal site}

The Bruchsal geothermal site is located at the eastern main boundary fault of the Upper Rhine Graben. The geothermal power plant consists of a borehole doublet: an injection well (GB1) and a production well (GB2) located at a distance of $1.5 \mathrm{~km}$ from each other (Fig. 2.1).
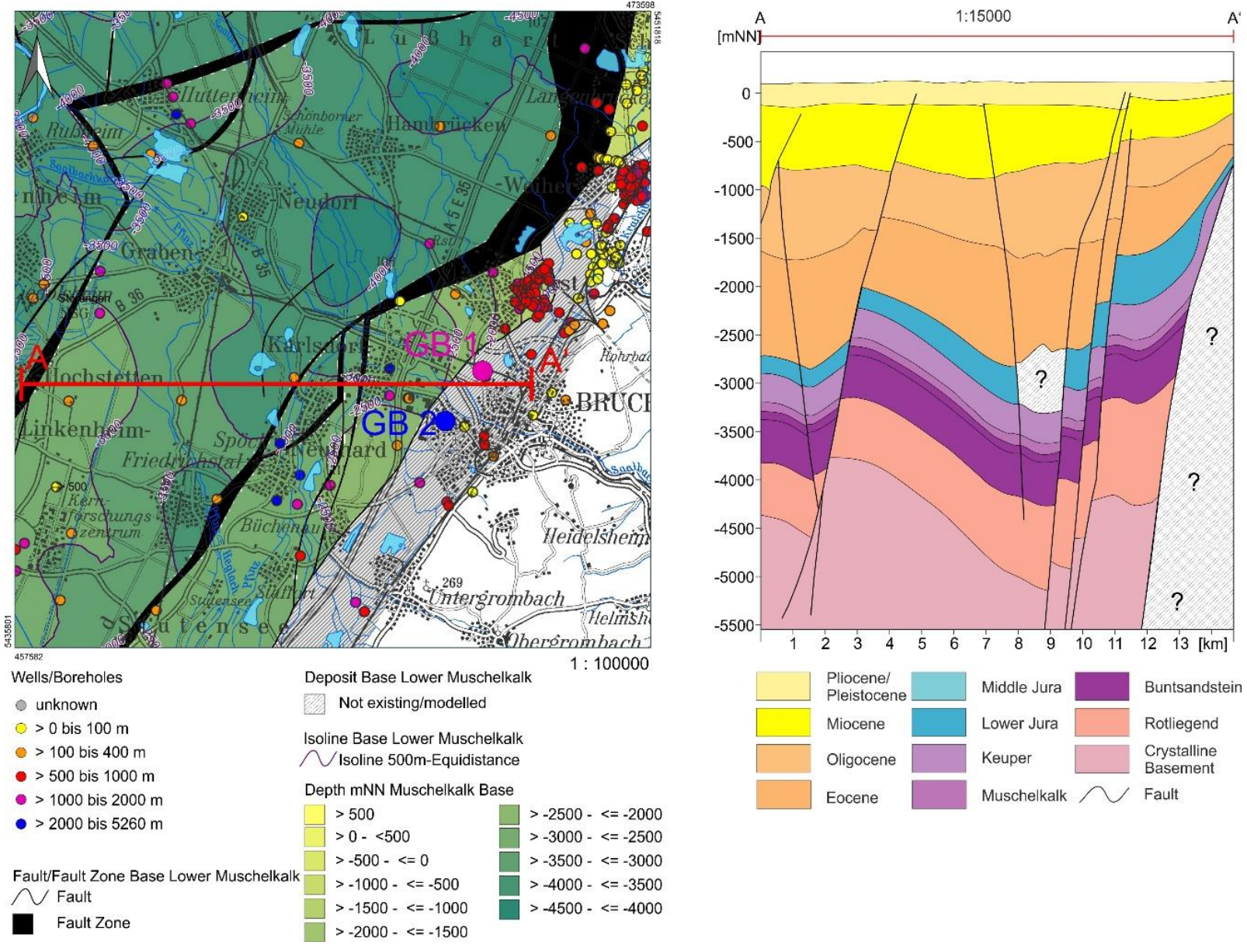

Fig. 2.1: Tectonic map of the study area (left) and cross section (right) modified after LGRB (2020). GB1 and GB2 represent the Bruchsal injection and production well, respectively. 
Because of the complex tectonic structure, the reservoir section at the injection well differs from that of the production well in depth and thickness. The geothermal reservoir is located in PermoTriassic sedimentary rocks, the structural setting is characterized by large-scale normal faults of varying step heights, ranging between 20 and $350 \mathrm{~m}$. The hydrothermal reservoir is developed in a horizon comprising Upper Rotliegend to Middle Buntsandstein rocks (depth interval: 2220-2485 $\mathrm{m})$. The main inflow zones are located in the fractured zones of the Upper Permian at a depth interval between 2440 and $2470 \mathrm{~m}$ (Joachim et al., 1987).

Borehole data of GB2 presented an undisturbed bottom hole temperature of $134.7^{\circ} \mathrm{C}$ at $2542 \mathrm{~m}$ depth, corresponding to a geothermal gradient of $55 \mathrm{~K} \mathrm{~km}^{-1}$, which exceeds the typical average value for Central Europe of $30 \mathrm{~K} \mathrm{~km}^{-1}$. At present, the geothermal brine is produced with a volume flow of $28 \mathrm{~L} / \mathrm{s}$. Geochemical analysis of the $\mathrm{NaCl}$ brine showed high mineralization (TDS $\approx 130$ $\mathrm{g} / \mathrm{l}$ ) including heavy metals and gases at a pH of 5 (Fritz et al., 1989; Rettenmaier et al., 2013).

\subsection{Bruchsal radiochemical monitoring system}

Natural radioactivity may affect the operation of a geothermal power plant in several ways. In geothermal energy systems, a huge quantity of geothermal fluid is circulated in the reservoir during the exploitation phase and generates interaction between surface geothermal fluid and host rock potentially capable of modifying natural radionuclides exchanges. Because of changing temperature and pressure during the passage of the produced fluid through the geothermal power plant, precipitations within the surface installation parts can be observed (e.g. Scheiber et al., 2012; Rettenmaier et al., 2013). The content of radioactivity varies according to the deposit's mineralogy.

\subsubsection{Setup of the sampling system}

The Bruchsal monitoring system was realized in the framework of the ANEMONA research project funded by Federal Ministry for Economic Affairs and Energy (BMWi). The project focused on chemical and radiochemical analysis of the geothermal brine, the gas phase and solids within the surface installation parts.

In terms of sampling, the best positioning of the sampling points depends on the types of sample (liquid, gas or solid). To meet these demands, the existing on-site sampling system was reconstructed. In a first step, new positions for the sampling points have been chosen to map all relevant processes that might change the fluid composition; beginning with the wellhead of the production (Fig. 2.2). In a second step, all sampling points were equipped with three sampling taps located at the top and at the bottom as well as in a lateral position of the pipe (Fig. 2.3). 


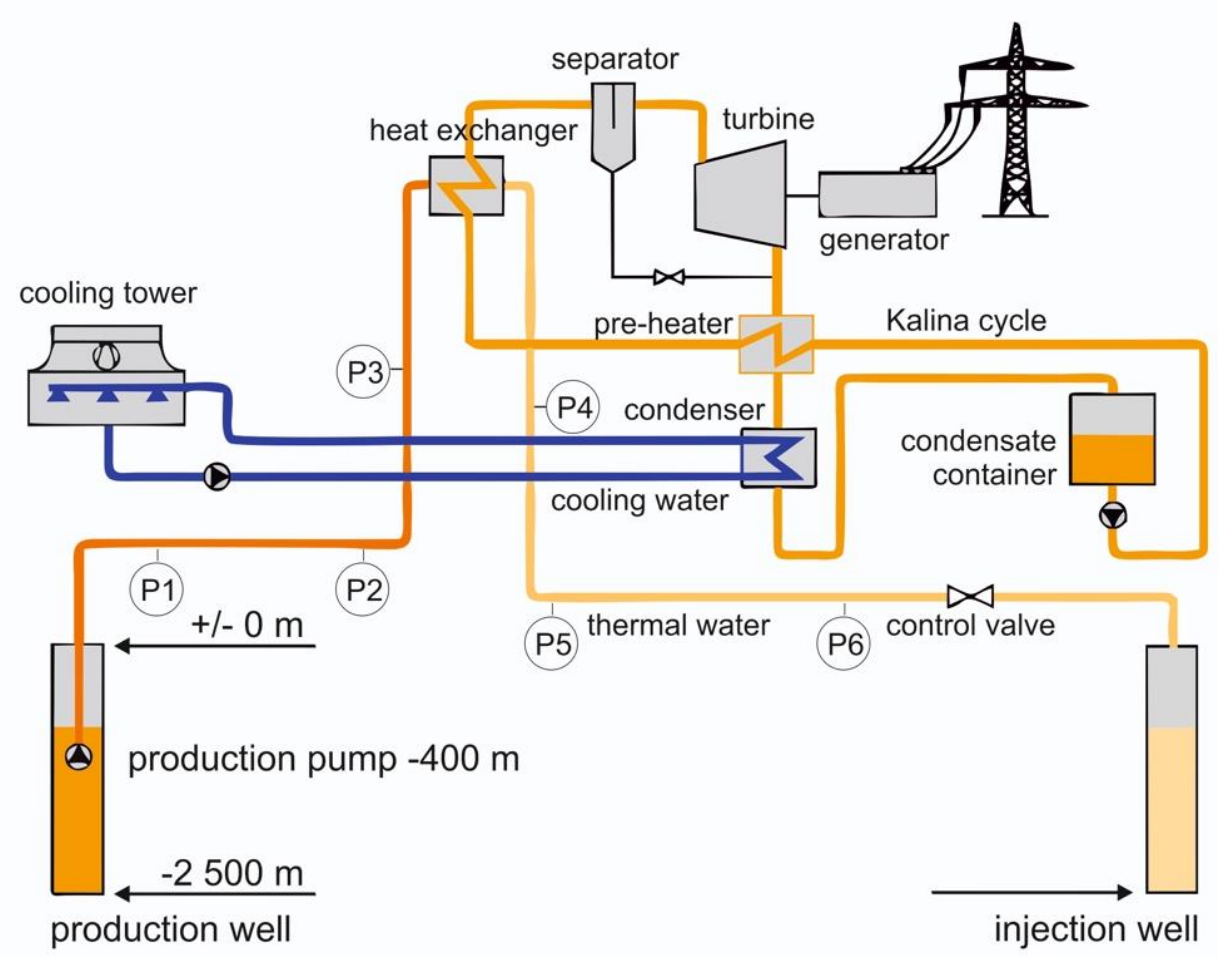

Fig. 2.2: Scheme of Bruchsal surface installation with the sampling points (P1-P6) of the monitoring network (according to Eggeling et al., 2018).


Fig. 2.3: Sampling point after reconstruction: the pipe is equipped with three sampling taps arranged at angels of $0^{\circ}$, $120^{\circ}$ und $180^{\circ}$ (left) and cross-sectional view of the sampling tabs' positioning within the pipe (right) (according to Eggeling et al., 2018). 
The latter is used for water sampling. Solid particles which were deposited at the cube sheet are filtered from the sampling tap at the bottom. Since the upper pipe section is filled with free gases at Bruchsal site, gas samples are taken from the tap at the top.

Within the scope of this thesis, the sampling point close to the wellhead (P1) are of major interest since the brine composition is closest to those in the reservoir. However, brine composition may already differ in response to variations of pressure, temperature, redox potential, and $\mathrm{pH}$ value while rising from reservoir to wellhead.

The sampling period spanned between October 2016 and Mai 2017. In total 32 water samples were collected. Major ions and selected trace elements were analyzed in the samples by ICP-OES, ion chromatography and photometry. Temperature, $\mathrm{pH}$, and conductivity were measured on-site. ${ }^{238} \mathrm{U}$, ${ }^{232} \mathrm{Th}$ and ${ }^{235} \mathrm{U}$ decay series radionuclides were analyzed in thirteen samples to study their temporal variations in activities. Radionuclide sampling was conducted using gas-tight 1.2-liter Marinelli beakers (type G-130 G) prepared with $2.5 \mathrm{ml} 65 \% \mathrm{HNO}_{3}$ (suprapur) to inhibit precipitation of solids. Water samples were not filtered.

\subsubsection{Parameter selection}

A number of sampling parameters such as sampling locations and frequency, type and quantity of samples as well as the sampling equipment have been specified, gathered and subsequently analyzed and evaluated. The parameters selected and the associated test methods used are listed in Table 2.1 .

Table 2.1: Measuring parameters and the related test methods used for the Bruchsal fluid monitoring.

\begin{tabular}{|c|c|c|c|}
\hline Physical parameter & Symbol & Unit & Testing method \\
\hline Sampling temperature & $T$ & ${ }^{\circ} \mathrm{C}$ & (DIN 38404:1976-12 (C4)) \\
\hline In situ conductivity & $L F$ & $\mu \mathrm{S} \mathrm{cm} \mathrm{cm}^{-1}$ & (DIN EN 27888:1993-11 (C8)) \\
\hline Conductivity at $25^{\circ} \mathrm{C}$ & $L F$ & $\mu \mathrm{S} \mathrm{cm} \mathrm{cm}^{-1}$ & (DIN EN 27888:1993-11 (C8)) \\
\hline In situ $\mathrm{pH}$ & $p H$ & & (DIN EN ISO 10523:2012-04 (C5)) \\
\hline $\mathrm{pH}$ at $20^{\circ} \mathrm{C}$ & $p H$ & & (DIN EN ISO 10523:2012-04 (C5)) \\
\hline Redox potential & $E h$ & $\mathrm{mV}$ & (DIN 38404:1984-05 (C6)) \\
\hline Dissolved oxygen & $\mathrm{O}_{2}$ & $\mathrm{mg} \mathrm{L}^{-1}$ & (DIN 38408:1984 (G22)) \\
\hline Density at $20^{\circ} \mathrm{C}$ & $\varrho$ & $\mathrm{kg} \mathrm{m}^{-3}$ & (DIN 38408:1984 (C9)) \\
\hline Sensory parameters & & & Testing method \\
\hline Turbidity (visual) & & & (DIN EN I38404-2:1976-12 (C2)) \\
\hline Smell & & & (DEV-B 1/2:1971) \\
\hline Color & & & (DIN EN ISO 7887:2011) \\
\hline
\end{tabular}




\begin{tabular}{|c|c|c|c|}
\hline Major ions & Symbol & Unit & Testing method \\
\hline Lithium & $\mathrm{Li}^{+}$ & $\mathrm{mg} \mathrm{L} \mathrm{L}^{-1}$ & (DIN EN ISO 11885:2009-09 (E22)) \\
\hline Sodium & $\mathrm{Na}^{+}$ & $m g L^{-1}$ & (DIN EN ISO 11885:2009-09 (E22)) \\
\hline Potassium & $K^{+}$ & $\mathrm{mg} \mathrm{L} \mathrm{L}^{-1}$ & (DIN EN ISO 11885:2009-09 (E22)) \\
\hline Calcium & $C a^{2+}$ & $\mathrm{mg} \mathrm{L} \mathrm{L}^{-1}$ & (DIN EN ISO 11885:2009-09 (E22)) \\
\hline Magnesium & $M g^{2+}$ & $m g L^{-1}$ & (DIN EN ISO 11885:2009-09 (E22)) \\
\hline Strontium & $S r^{2+}$ & $\mathrm{mg} \mathrm{L} \mathrm{L}^{-1}$ & (DIN EN ISO 11885:2009-09 (E22)) \\
\hline Barium & $B a^{2+}$ & $m g L^{-1}$ & (DIN EN ISO 11885:2009-09 (E22)) \\
\hline Hydrogen carbonate & $\mathrm{HCO}_{3}^{-}$ & $m g L^{-1}$ & (DEV D8, 1971) \\
\hline Chloride & $C t$ & $\mathrm{mg} \mathrm{L} \mathrm{L}^{-1}$ & (DIN EN ISO 10304-1:2009-07) \\
\hline Sulfate & $\mathrm{SO}_{4}^{2-}$ & $\operatorname{mg~L} L^{-1}$ & (DIN EN ISO 10304-1:2009-07) \\
\hline Nitrate & $\mathrm{NO}^{3-}$ & $\mathrm{mg} \mathrm{L} \mathrm{L}^{-1}$ & (DIN EN ISO 10304-1:2009-07) \\
\hline Bromide & $\mathrm{Br}$ & $\mathrm{mg} \mathrm{L} \mathrm{L}^{-1}$ & (DIN EN ISO 10304-1:2009-07) \\
\hline Fluoride & $F^{-}$ & $\mathrm{mg} \mathrm{L} \mathrm{L}^{-1}$ & (DIN EN ISO 10304-1:2009-07) \\
\hline Sulfide total & $H S^{-}, S^{2-}$ & $\mathrm{mg} \mathrm{L} \mathrm{L}^{-1}$ & (DIN 38405-27:2017-10 (D27)) \\
\hline Trace elements & Symbol & Unit & Testing method \\
\hline Aluminum & $A l$ & $\mathrm{mg} \mathrm{L} \mathrm{L}^{-1}$ & (DIN EN ISO 11885:2009-09 (E22)) \\
\hline Antimony & $S b$ & $\mathrm{mg} \mathrm{L} \mathrm{L}^{-1}$ & (DIN EN ISO 11885:2009-09 (E22)) \\
\hline Arsenic total & As & $\mathrm{mg} \mathrm{L} \mathrm{L}^{-1}$ & (DIN EN ISO 11885:2009-09 (E22)) \\
\hline Lead & $\mathrm{Pb}$ & $\mathrm{mg} \mathrm{L} \mathrm{L}^{-1}$ & (DIN EN ISO 11885:2009-09 (E22)) \\
\hline Cadmium & $C d$ & $\mathrm{mg} \mathrm{L} \mathrm{L}^{-1}$ & (DIN EN ISO 11885:2009-09 (E22))) \\
\hline Cesium & Cs & $\mathrm{mg} \mathrm{L} \mathrm{L}^{-1}$ & (DIN EN ISO 11885:2009-09 (E22))) \\
\hline Iron total & $F e_{t o t}$ & $\mathrm{mg} \mathrm{L} \mathrm{L}^{-1}$ & $\begin{array}{l}\text { (DIN EN ISO 11885:2009-09 (E22)) } \\
\text { (DIN 38406-1:1983-5 (E1)) }\end{array}$ \\
\hline Iron-II & $\mathrm{Fe}^{2+}$ & $\mathrm{mg} \mathrm{L} \mathrm{L}^{-1}$ & (DIN 38406-1:1983-5 (E1)) \\
\hline Iron-III & $\mathrm{Fe}^{3+}$ & $\mathrm{mg} \mathrm{L} \mathrm{L}^{-1}$ & (DIN 38406-1:1983-5 (E1)) \\
\hline Copper & $\mathrm{Cu}$ & $\operatorname{mg~L} \mathrm{L}^{-1}$ & (DIN EN ISO 11885:2009-09 (E22)) \\
\hline Manganese total & $M n_{t o t}$ & $\mathrm{mg} \mathrm{L} \mathrm{L}^{-1}$ & (DIN EN ISO 11885:2009-09 (E22)) \\
\hline Mercury & $H g$ & $\mathrm{mg} \mathrm{L} \mathrm{L}^{-1}$ & (DIN EN ISO 11885:2009-09 (E22)) \\
\hline Rubidium & $\mathrm{R} b$ & $\mathrm{mg} \mathrm{L} \mathrm{L}^{-1}$ & (DIN EN ISO 17294-2:2017-01) \\
\hline Zinc & $Z n$ & $\mathrm{mg} \mathrm{L} \mathrm{L}^{-1}$ & (DIN EN ISO 11885:2009-09 (E22)) \\
\hline Undissociated parameters & Symbol & Unit & Testing method \\
\hline Silica total & $\mathrm{SiO}_{2}$ & $\mathrm{mg} \mathrm{L} \mathrm{L}^{-1}$ & (DIN 38405-21:1990-5 (D21)) \\
\hline Organic matters & Symbol & Unit & Testing method \\
\hline
\end{tabular}




\begin{tabular}{|c|c|c|c|}
\hline Dissolved organic carbon & $D O C$ & $\mathrm{mg} \mathrm{L} \mathrm{L}^{-1}$ & (DIN EN 1486:1997) \\
\hline Radionuclides & Symbol & Unit & Testing method \\
\hline Thorium-232 & ${ }^{232} \mathrm{Th}$ & $\mathrm{Bq} \mathrm{L} \mathrm{L}^{-1}$ & Cf. chapter 2.5 \\
\hline Radium-228 & ${ }^{228} \mathrm{R} a$ & $\mathrm{~Bq} \mathrm{~L}^{-1}$ & Cf. chapter 2.5 \\
\hline Thorium-228 & ${ }^{228} \mathrm{Th}$ & $\mathrm{Bq} \mathrm{L}^{-1}$ & Cf. chapter 2.5 \\
\hline Radium-224 & ${ }^{224} \mathrm{R} a$ & $\mathrm{~Bq} \mathrm{~L} \mathrm{~L}^{-1}$ & Cf. chapter 2.5 \\
\hline Uranium-238 & ${ }^{238} \mathrm{U}$ & $\mathrm{Bq} \mathrm{L}^{-1} 1$ & Cf. chapter 2.5 \\
\hline Uranium-234 & ${ }^{234} U$ & $\mathrm{~Bq} \mathrm{~L}^{-1}$ & Cf. chapter 2.5 \\
\hline Thorium-230 & ${ }^{230} \mathrm{Th}$ & $\mathrm{Bq} \mathrm{L}^{-1}$ & Cf. chapter 2.5 \\
\hline Radium-226 & ${ }^{226} \mathrm{R} a$ & $\mathrm{~Bq} \mathrm{~L}^{-1}$ & Cf. chapter 2.5 \\
\hline Lead-210 & ${ }^{210} \mathrm{~Pb}$ & $\mathrm{~Bq} \mathrm{~L}^{-1}$ & Cf. chapter 2.5 \\
\hline Uranium-235 & ${ }^{235} \mathrm{U}$ & $\mathrm{Bq} \mathrm{L}^{-1}$ & Cf. chapter 2.5 \\
\hline Thorium-227 & ${ }^{227} \mathrm{Th}$ & $\mathrm{Bq} \mathrm{L}^{-1}$ & Cf. chapter 2.5 \\
\hline Radium-223 & ${ }^{223} \mathrm{R} a$ & $\mathrm{~Bq} \mathrm{~L}^{-1}$ & Cf. chapter 2.5 \\
\hline Potassium-40 & ${ }^{40} \mathrm{~K}$ & $\mathrm{~Bq} \mathrm{~L}^{-1}$ & Cf. chapter 2.5 \\
\hline
\end{tabular}

\subsection{Supportive analysis and data acquisition}

In addition to the fluid monitoring system, information about the solid phase of the geothermal reservoir can be gained by analyzing drill cuttings and geophysical borehole logs. Both data sources are available from the Bruchsal geothermal wells.

Since the modelling of radioactive disequilibria requires both types of information, the composition of the fluid and the solid phase, the radionuclide signature of the reservoir rock becomes vital.

Furthermore, hydrothermal alteration processes may result from the interaction of the geothermal fluid with the associated solids in fluid circulation zones. Since fractured zones provide a substantial portion of the total permeability due to the low porosity of the Bruchsal sandstone reservoir rock, alteration minerals are linked to reservoir sections with an increased hydraulic permeability. Assuming that the distribution of Th-U series nuclides is affected by hydrothermal processes, productive zones might be therefore indicated by specific radionuclide signatures.

To analyze these issues in detail, investigations of the Bruchsal reservoir rock based on the drill cuttings was executed. First, a petrographic alteration study was performed aiming the identification of hydrothermally altered minerals. Thin sections of $30 \mu \mathrm{m}$ thickness were prepared from the GB2 drill cuttings and investigated by optical polarizing microscopy. In addition, selected thin sections were subsequently coated with carbon and examined by scanning electron microscope (FEI QUANTA 650). 
Alpha- and gamma-ray measurements of the Bruchsal drill cuttings deliver specific activities of the Th-U series nuclides. The measuring results provide information about the state of equilibrium of the natural decay series inside the rock suites. The measuring method for the quantitative determination of Th-U series nuclides is described in detail in chapter 2.5 .

The fluid circulation and physico-chemical processes in geothermal systems can also affect the physical properties of the subsurface causing measurable anomalies in a number of state variables. Therefore, physical parameters such as temperature, electrical conductivity, permeability of the rock material and others are key indicators for the detection of geothermal reservoir. The following borehole logs are available for the deeper borehole sections of the GB2 production well at Bruchsal site: natural gamma ray (NGR), deep and shallow formation resistivity tool (DLL), caliper log (CAL) and temperature log (TEMP). Details about the methodological well logging techniques can be found in Ellis and Singer (2007).

\subsection{Spetrometry surveys of Th-U series nuclides}

Alpha- and gamma-ray measurements of water and rock samples provide information about possible radioactive disequilibria not only in the geothermal fluid but also in the adjoining solids.

The difference between alpha and gamma spectrometry is that the latter allows simultaneous measurements without further sample preparation. This is a huge advantage in terms of on-site, nearly real time measurements, for example, the evaluation of rock material during drilling operations. The method gives results for the gamma-ray emitting Th-U series radionuclides from decay and ingrowth of their progeny, respectively. In geothermal systems, however, isotopes of thorium and uranium can have activity below the device-specific limit of quantification of the gamma spectrometry, but also of alpha spectrometry. Table 2.2 summarizes the radionuclides investigated and the analytical methods applied.

\subsubsection{Gamma spectrometry}

Gamma spectrometric measurements utilize the method of pulse height analysis allowing the direct determination of individual radionuclides in gamma-emitting samples. This non-destructive method gives results for the gamma-ray emitting radionuclides ${ }^{238} \mathrm{U},{ }^{235} \mathrm{U},{ }^{226} \mathrm{Ra},{ }^{210} \mathrm{~Pb},{ }^{228} \mathrm{Ra}$ and

${ }^{228} \mathrm{Th}$ which are usually determined via the decay and ingrowth of short-lived daughters (except ${ }^{210} \mathrm{~Pb}$ and ${ }^{235} \mathrm{U}$ ) (Ivanovich and Murray, 1992).

There are several detector geometries available, all basing on high-purity germanium detectors (HPGe). It is distinguished between "n-type" and "p-type" of HPGe detectors depending on the concentration of donor or acceptor atoms in the crystal. 
Table 2.2: Alpha-ray and gamma-ray energies $E_{a, y}(\mathrm{keV})$ and emission probability $P_{a}$ used for the determination of specific activities of Th-U series radionuclides. Data about $\alpha$-particle energy $\left(E_{a}\right)$ and emission probability $\left(P_{a}\right)$ are reported by Rytz (1991) while data about gamma-ray energy $\left(E_{\gamma}\right)$ and emission probability $\left(P_{\gamma}\right)$ are from Schötzig and Schrader (1998).

\begin{tabular}{|c|c|c|c|c|c|}
\hline $\begin{array}{l}\text { Decay } \\
\text { series }\end{array}$ & $\begin{array}{l}\text { Radionuclide } \\
\text { investigated }\end{array}$ & $\begin{array}{l}\text { Analytical } \\
\text { methods }\end{array}$ & $\begin{array}{l}\text { Measured radi- } \\
\text { onuclides }\end{array}$ & $\begin{array}{l}\text { Energy } \\
\mathrm{E}_{\alpha, \gamma}(\mathrm{keV})\end{array}$ & $\begin{array}{l}\text { Probability } \\
\mathrm{P}_{\alpha, \gamma}(\%)\end{array}$ \\
\hline \multirow{6}{*}{${ }^{238} \mathrm{U}$ series } & $\mathrm{U}-238$ & $\alpha$ & $\mathrm{U}-238$ & $4198.0 ; 4151.0$ & $77.0 ; 23.0$ \\
\hline & U-234 & $\alpha$ & U-234 & $4774.6 ; 4722.4$ & $72.5 ; 27.5$ \\
\hline & Th-230 & $\alpha$ & Th-230 & $4687.0 ; 4620.5$ & $76.3 ; 23.4$ \\
\hline & Ra-226 & $\alpha$ & 226-Ra & $4784.3 ; 4601.0$ & $94.5 ; 5.5$ \\
\hline & & $\gamma$ & $\mathrm{Pb}-214$ & $295.2 ; 242.0$ & 18.2; 7.1; \\
\hline & & $\gamma$ & $\mathrm{Bi}-214$ & $609.3 ; 1120.3 ; 1764.5$ & $44.6 ; 14.7 ; 15.1$ \\
\hline \multirow{5}{*}{${ }^{232} \mathrm{Th}$ series } & Th-232 & $\alpha$ & Th-232 & $4013.0 ; 3950.0$ & $77.0 ; 23.0$ \\
\hline & $\mathrm{Ra}-228$ & $\gamma$ & Ac-228 & $911.2 ; 969.0 ; 338.3$ & $25.8 ; 15.8 ; 11.3$ \\
\hline & Th-228 & $\alpha$ & Th-228 & $5423.2 ; 5340.4$ & $73.4 ; 26.6$ \\
\hline & $\mathrm{Ra}-224$ & $\gamma$ & $\mathrm{Pb}-212$ & 238.6 & 43.3 \\
\hline & & $\gamma$ & Tl-208 & 583.2 & 30.4 \\
\hline${ }^{235} \mathrm{U}$ series & Ra-223 & $\gamma$ & Ra-223 & $154.3 ; 269.6$ & $5.6 ; 13.7$ \\
\hline
\end{tabular}

The principal detector geometries are planar, coaxial, and well-type whereas coaxial detectors provide the highest photopeak efficiencies.

Since this thesis targets the migratory behavior of radium $\left({ }^{223} \mathrm{Ra},{ }^{224} \mathrm{Ra},{ }^{226} \mathrm{Ra},{ }^{228} \mathrm{Ra}\right)$ in geothermal systems, the following explains how to calculate $\mathrm{Ra}$ activities in environmental samples characterized by radioactive disequilibria.

The activity of ${ }^{226} \mathrm{Ra}$ can be directly measured using its gamma ray energy at $186.2 \mathrm{keV}$. A possible interference with ${ }^{235} \mathrm{U}(185.7 \mathrm{keV})$ is negligible due to the low uranium activity in deep geothermal brines. Alternatively, the gamma-peaks of ${ }^{214} \mathrm{~Pb}$ and ${ }^{214} \mathrm{Bi}$ (daughters of ${ }^{226} \mathrm{Ra}$ and ${ }^{222} \mathrm{Rn}$, respectively) can be used to calculate the ${ }^{226} \mathrm{Ra}$ activity. In this case, the measurement can be performed after 20 days at the earliest, because this time is required to reach secular radioactive equilibrium (assuming 
no radon loss).

$154.3 \mathrm{keV}$ gamma ray energy can be used to determine the ${ }^{223} \mathrm{Ra}$ activity. Here the presence of an interfering peak from ${ }^{228} \mathrm{Ac}$ must be considered. According to Condomines et al. (2010), ${ }^{223} \mathrm{Ra}$ activity based on the $154.3 \mathrm{keV}$ peak must be corrected by:

$$
\overline{\left({ }^{223} R a\right)}=\frac{C}{C_{S}} \cdot\left[\left({ }^{223} R a\right)_{S}+\left({ }^{228} A c\right)_{S} \cdot \frac{\varepsilon_{A c} I_{\gamma, A c}}{\varepsilon_{R a} I_{\gamma, R a}}\right]-\overline{\left({ }^{228} A c\right)} \cdot \frac{\varepsilon_{A c} I_{\gamma, A c}}{\varepsilon_{R a} I_{\gamma, R a}}
$$

where $\overline{\left({ }^{223} R a\right)}$ and $\overline{\left({ }^{228} A c\right)}$ are average activities integrated over the counting time. $C$ and $C_{S}$ are the counts for the sample and the standard, respectively. $\varepsilon_{A C}$ and $\varepsilon_{R a}$ are the apparent efficiencies for ${ }^{228} \mathrm{Ac}$ and ${ }^{223} \mathrm{Ra}$. $I_{\gamma, A c}$ and $I_{\gamma, R a}$ are gamma-ray intensities.

${ }^{228} \mathrm{Ra}$ and ${ }^{224} \mathrm{Ra}$ are part of the decay scheme of the ${ }^{232} \mathrm{Th}$ decay series. Measurements of both $\mathrm{Ra}$ isotopes include the count rates of their short-lived daughters ${ }^{228} \mathrm{Ac}\left(\mathrm{t}_{1 / 2}=6.13 \mathrm{~h}\right)$ and ${ }^{212} \mathrm{~Pb}\left(\mathrm{t}_{1 / 2}=\right.$ $10.64 \mathrm{~h}$ ) which evolve through time during counting. The time-dependent evolution of the thorium decay series radionuclides is illustrated in Fig. 2.4 and can be described by a system of coupled differential equations.

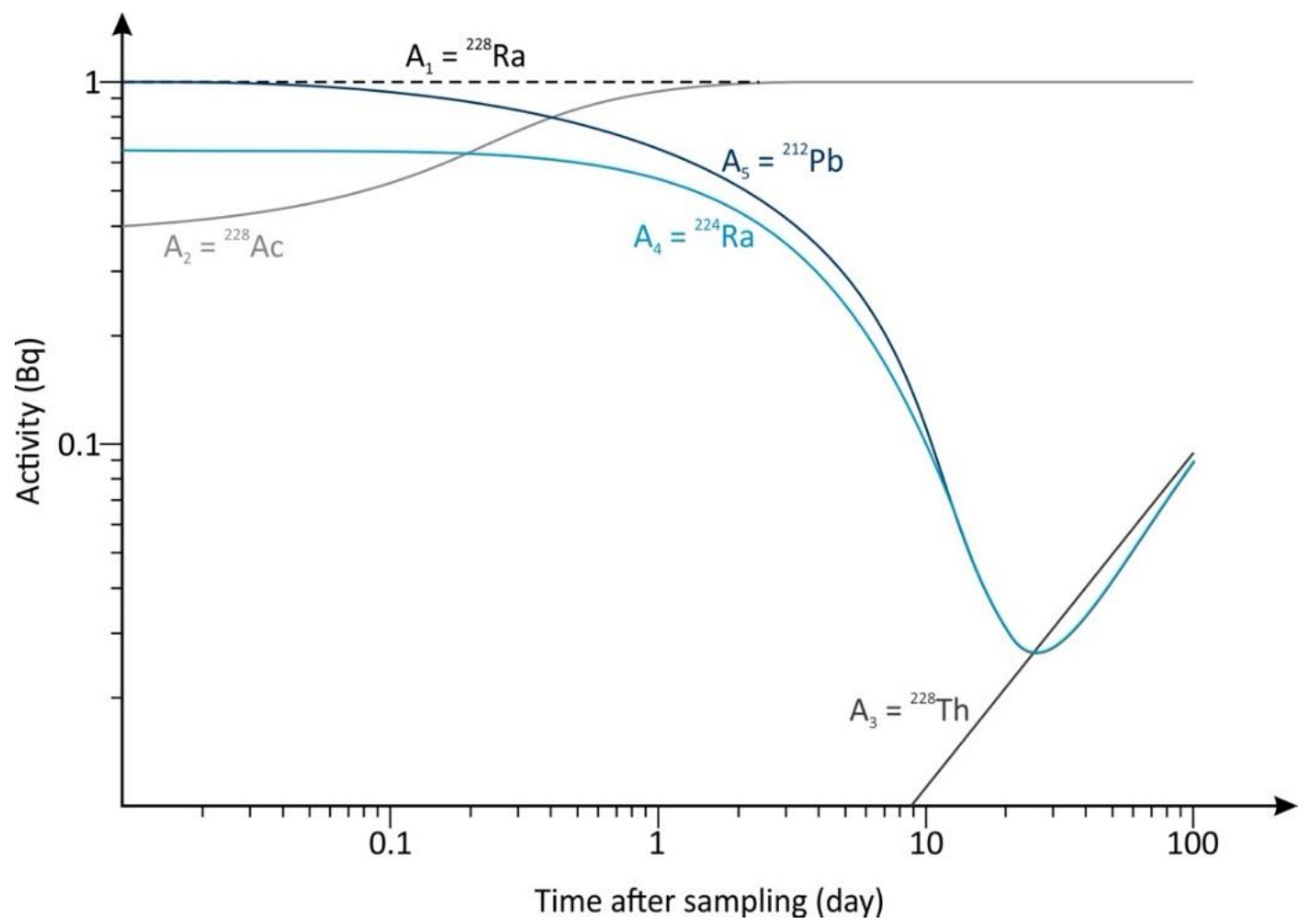

Fig. 2.4: Simulation of the time-depended evolution of standardized activity of ${ }^{228} \mathrm{Ra}$ and its daughters $\left({ }^{228} \mathrm{Ra} \rightarrow{ }^{228} \mathrm{Ac}\right.$ $\left.\rightarrow{ }^{228} \mathrm{Th} \rightarrow{ }^{224} \mathrm{Ra} \rightarrow{ }^{212} \mathrm{~Pb}\right)$. 
Their general solution was first given by Bateman (1910) who expressed the concentration of $n$th radionuclide after time $t$ as:

$$
N_{n}(t)=\frac{N_{1}(0)}{\lambda_{n}} \sum_{i=1}^{n} \lambda_{i} \alpha_{i} \exp \left[-\lambda_{i} t\right]
$$

where the coefficients are calculated by

$$
\alpha_{i}=\prod_{j=1}^{n} \frac{\lambda_{j}}{\left(\lambda_{j}-\lambda_{i}\right)} \quad(j \neq i)
$$

The mathematical solution for the Bruchsal site was computed by employing Mathcad $\AA$.

The activity of ${ }^{228} \mathrm{Ra}$ at sampling time $(\mathrm{t}=0)$ can be determined from the ${ }^{228}$ Ac activity after a waiting period $t_{d}$ of minimum 42 hours after sampling:

$$
A_{1}(0)=\frac{A_{2}\left(t_{d}, \Delta t\right)}{\tau_{1}\left(t_{d}, \Delta t\right)}
$$

${ }^{224} \mathrm{Ra}$ can be measured using its gamma-rays emitted daughter nuclides ${ }^{212} \mathrm{~Pb}(238.6 \mathrm{keV})$ and ${ }^{208} \mathrm{Tl}$ $(583.1 \mathrm{keV})$. The ${ }^{212} \mathrm{~Pb} /{ }^{224} \mathrm{Ra}$ ratio reach a steady state value of 1.14 after ca. 100 hours:

$$
\begin{aligned}
& A_{4}(0)=\frac{1}{\overline{\tau_{4}\left(t_{d}, \Delta t\right)}}\left(\overline{A_{4}\left(t_{d}, \Delta t\right)}-\left(c_{1} A_{1}(0)+c_{3} A_{3}(0)\right)\right. \\
& c_{1}=\alpha_{41} \alpha_{31}\left(\overline{\tau_{1}\left(t_{d}, \Delta t\right)}-\overline{\tau_{4}\left(t_{d}, \Delta t\right)}\right)-\alpha_{43} \alpha_{31}\left(\overline{\tau_{3}\left(t_{d}, \Delta t\right)}-\overline{\tau_{4}\left(t_{d}, \Delta t\right)}\right) \\
& c_{3}=\alpha_{43}\left(\overline{\tau_{3}\left(t_{d}, \Delta t\right)}-\overline{\tau_{4}\left(t_{d}, \Delta t\right)}\right) \\
& c_{4}=\overline{\tau_{4}\left(t_{d}, \Delta t\right)}
\end{aligned}
$$

where ${ }^{228} \mathrm{Ra}=\mathrm{A}_{1} ;{ }^{228} \mathrm{Ac}=\mathrm{A}_{2},{ }^{228} \mathrm{Th}=\mathrm{A}_{3}$ and ${ }^{224} \mathrm{Ra}=\mathrm{A}_{4}$.

\subsubsection{Alpha spectrometry}

One characteristic of alpha spectrometry of environmental samples is the generally very low activity of a few $\mathrm{mBq}$ to some $\mathrm{Bq}$ that needs to be detected. The measurement requires therefore devices with high counting efficiencies and low background radiation. An entire removal of the sample matrix is needed due to the small range of alpha particles in water and solids, respectively. Therefore, a radiochemical separation of the matrix and the radionuclide needs to be performed. 
A rather typical measurement principal used for alpha detection is the liquid scintillation counting (LSC). Moreover, high-resolution alpha-ray measurements can be performed using gas-filled ionizing detectors or semiconductor detectors. Further details dealing with the measurement technique and the alpha spectrometric determination of uranium and thorium isotopes in environmental samples can be found in Vajda et al., 2012.

\subsubsection{Uncertainties}

The determination of the characteristic limits (decisions threshold, detection limits and limits of the confidence interval) for measurements of ionizing radiation is specified in DIN ISO 11929. A distinction is made between type A uncertainty due to repeated measurements and statistical analysis and type B uncertainty. The latter includes, among other subjects, improper handling during sampling, inhomogeneity of the sample itself and geometry errors.

Type A and B uncertainties are relevant for the validation of the test method in terms of detection limits. Furthermore, the uncertainties need to be considered by evaluating the laboratory analysis results. In the simplest case, the measurement uncertainty corresponds to the simple standard deviation (without considering type B uncertainty.)

The expanded uncertainty of measurement is obtained by multiplying the standard measurement uncertainty by the coverage factor $\mathrm{k}=2$. For normal distribution, this coverage factor has a coverage probability of $95 \%$.

\subsection{Selected results}

The radiochemical fluid monitoring displays that the Bruchsal brine is in a state of radioactive disequilibria. Radium isotopes $\left({ }^{228} \mathrm{Ra},{ }^{226} \mathrm{Ra},{ }^{224} \mathrm{Ra},{ }^{223} \mathrm{Ra}\right)$ have activities far exceeding those of their thorium progenitors (average ${ }^{226} \mathrm{Ra}=29.9 \mathrm{~Bq} \mathrm{~kg}^{-1}$, about $10^{3}$ times that of its ${ }^{230} \mathrm{Th}$ parent).

The highest activities range from 27 to $32 \mathrm{~Bq} \mathrm{~kg}^{-1}$ and are identified for the long-lived ${ }^{226} \mathrm{Ra}$ isotope. ${ }^{228} \mathrm{Ra},{ }^{224} \mathrm{Ra}$ and ${ }^{223} \mathrm{Ra}$ activities show lower values that vary between 15 and $17 \mathrm{~Bq} \mathrm{~kg}^{-1}, 9$ and $12 \mathrm{~Bq}$ $\mathrm{kg}^{-1}$, and 0.3 and $0.7 \mathrm{~Bq} \mathrm{~kg}^{-1}$, respectively. Variation in activity levels of the radium isotopes are likely a consequence of analytical uncertainties and steady state in activities of the radium isotopes can be assumed.

${ }^{222} \mathrm{Rn}$ is in slight excess relative to its parent ${ }^{226} \mathrm{Ra}$. Isotopes of $\mathrm{Pb}\left({ }^{210} \mathrm{~Pb}\right.$ and $\left.{ }^{212} \mathrm{~Pb}\right)$ have high solubilities resulting in fluid activities $>15 \mathrm{~Bq} \mathrm{~kg}^{-1} \cdot{ }^{210} \mathrm{~Pb}\left(A_{210 \mathrm{~Pb}}=25.8 \mathrm{~Bq} \mathrm{~kg}^{-1}\right)$ was found to be deficient relative to its progenitors ${ }^{222} \mathrm{Rn}\left(A_{222 \mathrm{R} n}=37.8 \mathrm{~Bq} \mathrm{~kg}^{-1}\right)$ and ${ }^{226} \mathrm{Ra}\left(A_{226 \mathrm{R} a}=29.0 \mathrm{~Bq} \mathrm{~kg}{ }^{-1}\right)$, but still in the same order of magnitude indicating that $\mathrm{Ra}, \mathrm{Rn}$ and $\mathrm{Pb}$ have a comparable mobility in the Bruchsal geothermal system. In contrast, activities of Th $\left({ }^{230} \mathrm{Th},{ }^{232} \mathrm{Th}\right.$ and $\left.{ }^{228} \mathrm{Th}\right), \mathrm{U}\left({ }^{238} \mathrm{U},{ }^{234} \mathrm{U},{ }^{235} \mathrm{U}\right)$ 
and $\mathrm{Po}\left({ }^{210} \mathrm{Po}\right)$ isotopes in brine were below the limit of analytical determination. (Table 2.3).

Table 2.3: Specific activities of ${ }^{238} \mathrm{U},{ }^{232} \mathrm{Th}$ and ${ }^{235} \mathrm{U}$ decay series radionuclides dissolved in the Bruchsal brine. Relative uncertainties are quoted in percentage $( \pm 2 \sigma$ from counting statistics).

\begin{tabular}{|c|c|c|c|}
\hline Decay series & Radionuclide & $\begin{array}{l}\text { Specific activity } \\
\left(\mathrm{Bq} \mathrm{kg}^{-1}\right)\end{array}$ & $\begin{array}{l}\text { Relative uncertainty } \\
(\%)\end{array}$ \\
\hline \multirow{7}{*}{$\begin{array}{l}\text { Uranium } \\
\text { series }\end{array}$} & ${ }^{238} \mathrm{U}$ & 0.012 & 59 \\
\hline & ${ }^{234} \mathrm{U}$ & 0.01 & 66 \\
\hline & ${ }^{230} \mathrm{Th}$ & $<0.08$ & \\
\hline & ${ }^{226} \mathrm{Ra}$ & 29.0 & 8.3 \\
\hline & ${ }^{222} \mathrm{Rn}$ & 37.8 & 7.4 \\
\hline & ${ }^{210} \mathrm{~Pb}$ & 25.8 & 26 \\
\hline & ${ }^{210} \mathrm{Po}$ & $<0.1$ & \\
\hline \multirow{3}{*}{$\begin{array}{l}\text { Actinium } \\
\text { series }\end{array}$} & ${ }^{235} \mathrm{U}$ & $<0.004$ & \\
\hline & ${ }^{227} \mathrm{Ac}$ & $<0.1$ & \\
\hline & ${ }^{223} \mathrm{Ra}$ & 0.47 & 60 \\
\hline \multirow{6}{*}{$\begin{array}{l}\text { Thorium } \\
\text { series }\end{array}$} & ${ }^{232} \mathrm{Th}$ & $<0.01$ & \\
\hline & ${ }^{228} \mathrm{Ra}$ & 15.9 & 5.8 \\
\hline & ${ }^{228} \mathrm{Ac}$ & 6.1 & 27 \\
\hline & ${ }^{228} \mathrm{Th}$ & $<0.02$ & \\
\hline & ${ }^{224} \mathrm{Ra}$ & 10.3 & 7.5 \\
\hline & ${ }^{212} \mathrm{~Pb}$ & 15.7 & 6.4 \\
\hline
\end{tabular}

The isotopic ratios of radium are reported in Table $2.4 .{ }^{228} \mathrm{Ra} /{ }^{226} \mathrm{Ra},{ }^{224} \mathrm{Ra} /{ }^{228} \mathrm{Ra}$ and ${ }^{223} \mathrm{Ra} /{ }^{226} \mathrm{Ra}$ ratios display mean values of $0.55 \pm 0.07,0.65 \pm 0.07$ and $0.02 \pm 0.01$. Therefore, radium activity ratios are principally constant within $2 \sigma$ analytical uncertainties during the period of sampling.

Since the deep geothermal brine interacts with the adjoining reservoir rock, drill cuttings from the production well GB2 were also analyzed for their radium isotope signature. The mean ${ }^{228} \mathrm{Ra} /{ }^{226} \mathrm{Ra}$ activity ratio of the Lower Buntsandstein and Zechstein is approximately 0.54 . This is in good agreement with the observed ${ }^{228} \mathrm{Ra} /{ }^{226} \mathrm{Ra}$ signature of 0.55 in the Bruchsal geothermal fluid. 
Table 2.4: Radium activities and activity ratios in brine collected from GB2 well (measurement campaign 2016/2017). Measured values are reported in $\mathrm{Bq} \mathrm{kg}^{-1}$. The quoted errors are $2 \sigma$-deviations derived from counting statistics.

\begin{tabular}{|c|c|c|c|c|c|}
\hline $\begin{array}{l}\text { Sample } \\
\text { No. }\end{array}$ & $\begin{array}{l}\text { Sampling } \\
\text { date }\end{array}$ & ${ }^{226} \mathrm{Ra}$ & ${ }^{228} \mathrm{Ra} /{ }^{226} \mathrm{Ra}$ & ${ }^{224} \mathrm{Ra} /{ }^{228} \mathrm{Ra}$ & ${ }^{223} \mathrm{Ra} /{ }^{226} \mathrm{Ra}$ \\
\hline GB2-p1z1-001 & 13.10 .2016 & $\begin{array}{l}28.8 \\
\pm 2.4\end{array}$ & $\begin{array}{l}0.53 \\
\pm 0.07\end{array}$ & $\begin{array}{l}0.70 \\
\pm 0.08\end{array}$ & $\begin{array}{l}0.01 \\
\pm 0.01\end{array}$ \\
\hline GB2-p1z1-002 & 18.10 .2016 & $\begin{array}{l}27.6 \\
\pm 2.6\end{array}$ & $\begin{array}{l}0.54 \\
\pm 0.08\end{array}$ & $\begin{array}{l}0.67 \\
\pm 0.06\end{array}$ & $\begin{array}{l}0.01 \\
\pm 0.01\end{array}$ \\
\hline GB2-p1z1-003 & 24.10 .2016 & $\begin{array}{l}28.1 \\
\pm 2.4\end{array}$ & $\begin{array}{l}0.55 \\
\pm 0.08\end{array}$ & $\begin{array}{l}0.67 \\
\pm 0.06\end{array}$ & $\begin{array}{l}0.02 \\
\pm 0.01\end{array}$ \\
\hline GB2-p1z1-004 & 28.10 .2016 & $\begin{array}{l}29.3 \\
\pm 2.8\end{array}$ & $\begin{array}{l}0.56 \\
\pm 0.08\end{array}$ & $\begin{array}{l}0.70 \\
\pm 0.08\end{array}$ & $\begin{array}{l}0.02 \\
\pm 0.01\end{array}$ \\
\hline GB2-p1z1-005 & 04.11 .2016 & $\begin{array}{l}27.2 \\
\pm 2.2\end{array}$ & $\begin{array}{l}0.59 \\
\pm 0.08\end{array}$ & $\begin{array}{l}0.56 \\
\pm 0.15\end{array}$ & $\begin{array}{l}0.03 \\
\pm 0.02\end{array}$ \\
\hline GB2-p1z1-006 & 16.11.2016 & $\begin{array}{l}28.0 \\
\pm 2.4\end{array}$ & $\begin{array}{l}0.55 \\
\pm 0.08\end{array}$ & $\begin{array}{l}0.68 \\
\pm 0.07\end{array}$ & $\begin{array}{l}0.02 \\
\pm 0.01\end{array}$ \\
\hline GB2-p1z1-007 & 17.01.2017 & $\begin{array}{l}29.5 \\
\pm 2.4\end{array}$ & $\begin{array}{l}0.53 \\
\pm 0.07\end{array}$ & $\begin{array}{l}0.65 \\
\pm 0.09\end{array}$ & $\begin{array}{l}0.02 \\
\pm 0.01\end{array}$ \\
\hline GB2-p1z1-008 & 04.04 .2017 & $\begin{array}{l}30.3 \\
\pm 2.2\end{array}$ & $\begin{array}{l}0.53 \\
\pm 0.06\end{array}$ & $\begin{array}{l}0.64 \\
\pm 0.05\end{array}$ & $\begin{array}{l}0.01 \\
\pm 0.01\end{array}$ \\
\hline GB2-p1z1-009 & 11.04 .2017 & $\begin{array}{l}32.0 \\
\pm 2.4\end{array}$ & $\begin{array}{l}0.52 \\
\pm 0.07\end{array}$ & $\begin{array}{l}0.58 \\
\pm 0.09\end{array}$ & $\begin{array}{l}0.01 \\
\pm 0.01\end{array}$ \\
\hline GB2-p1z1-010 & 27.04 .2017 & $\begin{array}{l}29.9 \\
\pm 2.4\end{array}$ & $\begin{array}{l}0.54 \\
\pm 0.07\end{array}$ & $\begin{array}{l}0.66 \\
\pm 0.07\end{array}$ & $\begin{array}{l}0.02 \\
\pm 0.01\end{array}$ \\
\hline GB2-p1z1-011 & 02.05 .2017 & $\begin{array}{l}27.8 \\
\pm 2.4\end{array}$ & $\begin{array}{l}0.59 \\
\pm 0.08\end{array}$ & $\begin{array}{l}0.65 \\
\pm 0.06\end{array}$ & $\begin{array}{l}0.02 \\
\pm 0.01\end{array}$ \\
\hline GB2-p1z1-012 & 08.05 .2017 & $\begin{array}{l}28.9 \\
\pm 2.4\end{array}$ & $\begin{array}{l}0.56 \\
\pm 0.07\end{array}$ & $\begin{array}{l}0.69 \\
\pm 0.06\end{array}$ & $\begin{array}{l}0.02 \\
\pm 0.01\end{array}$ \\
\hline GB2-p1z1-013 & 15.05 .2017 & $\begin{array}{l}30.0 \\
\pm 2.4\end{array}$ & $\begin{array}{l}0.54 \\
\pm 0.07\end{array}$ & $\begin{array}{l}0.63 \\
\pm 0.06\end{array}$ & $\begin{array}{l}0.02 \\
\pm 0.01\end{array}$ \\
\hline
\end{tabular}


Isotope ratios within the ${ }^{238} \mathrm{U}$ decay series reveal a ${ }^{226} \mathrm{Ra}$ anomaly within the Lower Buntsandstein and Zechstein section (Fig. 2.5). Since ${ }^{234} \mathrm{U} /{ }^{238} \mathrm{U}$ and ${ }^{230} \mathrm{Th} /{ }^{234} \mathrm{U}$ activity ratios are equal to unity, ${ }^{226} \mathrm{Ra} /{ }^{230} \mathrm{Th}$ activity ratios are higher than unity indicate general disequilibria with values ranging from a minimum of 1.7 to a maximum of 3.3 .
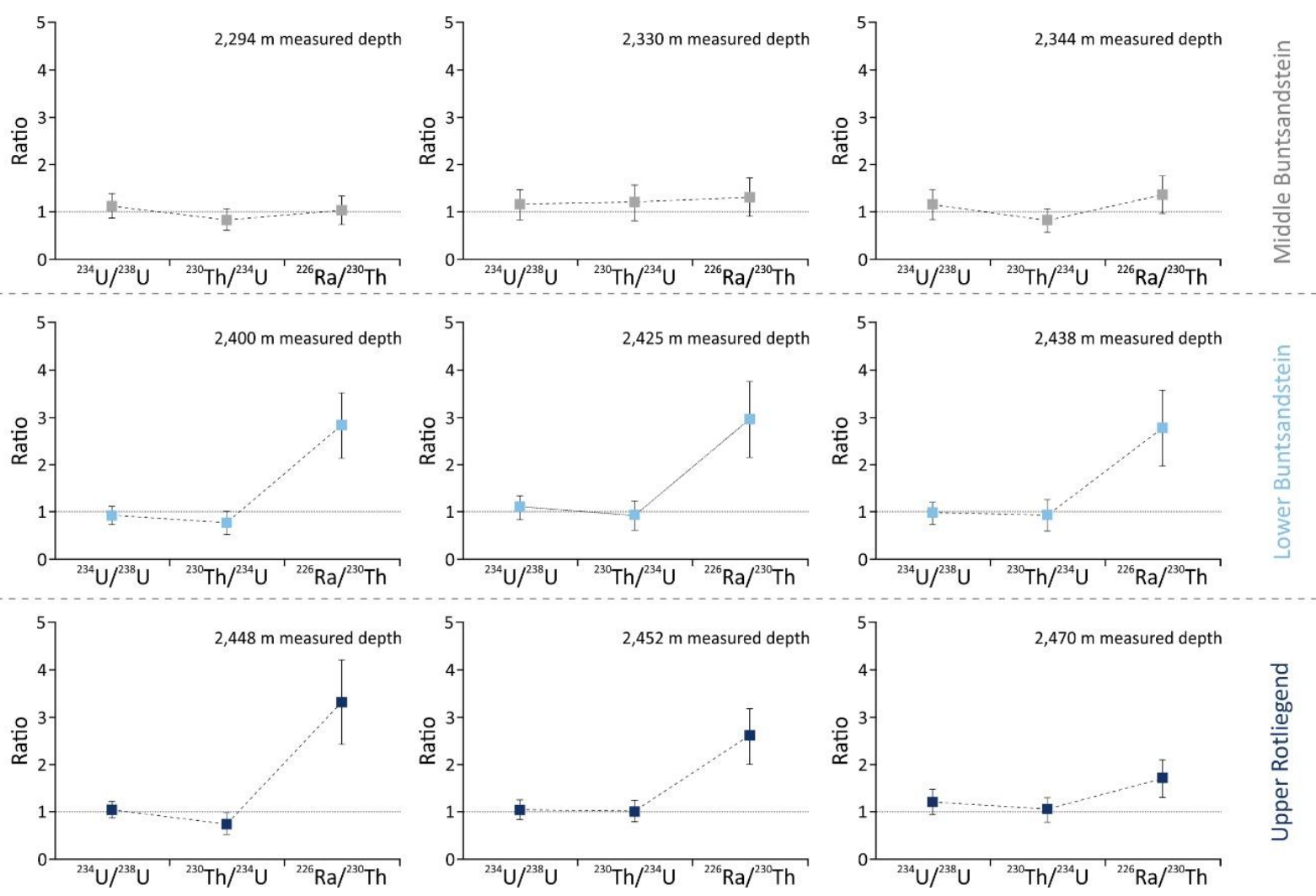

Fig. 2.5: Radionuclide disequilibrium within the reservoir section of GB2: cuttings of the Lower Buntsandstein and the older Permian Zechstein are significantly enriched with respect to ${ }^{226} \mathrm{Ra}$. Error bars represent uncertainties due to counting statistics $( \pm 1 \sigma)$.

Here, the ${ }^{226} \mathrm{Ra}$ activity exceeds the specific activity of ${ }^{238} \mathrm{U}$ and its decay products ${ }^{234} \mathrm{U}$ and ${ }^{230} \mathrm{Th}$ by a factor of 2 to 3 . The high ${ }^{226} \mathrm{Ra} /{ }^{230} \mathrm{Th}$ activity ratios in the deeper rock samples show that radium has been highly concentrated in the solid phase relative to thorium.

Table 2.5 summarizes the specific activities of ${ }^{232}$ Th series radionuclides measured in the GB2 cuttings. The average ${ }^{232} \mathrm{Th}$ activity in drill cutting of the Middle and Lower Buntsandstein is about 12 $\pm 2 \mathrm{~Bq} \mathrm{~kg}^{-1}$. Zechstein samples, however, show substantially higher activities of ${ }^{232} \mathrm{Th}$, up to $26 \pm$ $4 \mathrm{~Bq} \mathrm{~kg}^{-1}$. In contrast to the uranium decay series, there is no radioactive disequilibrium observed for the thorium decay series radionuclides. Activity ratios under investigation $\left({ }^{228} \mathrm{Ra} /{ }^{232} \mathrm{Th}\right.$, ${ }^{228} \mathrm{Th} /{ }^{228} \mathrm{Ra}$, and the ${ }^{224} \mathrm{Ra} /{ }^{228} \mathrm{Th}$ ) are close to unity. 
Table 2.5: Specific activity of ${ }^{232} \mathrm{Th}$ series nuclides measured in the GB2 drill cuttings. The analytical error is ca. $\pm 1 \sigma$.

\begin{tabular}{|c|c|c|c|c|c|c|c|}
\hline & & Sample & Depth period & ${ }^{232} \mathrm{Th}$ & ${ }^{228} \mathrm{Ra}$ & ${ }^{228} \mathrm{Th}$ & ${ }^{224} \mathrm{Ra}$ \\
\hline \multirow{6}{*}{ 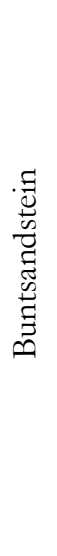 } & \multirow{3}{*}{$\frac{\ddot{\theta}}{\stackrel{7}{\theta}}$} & 1 & $2,330-2,335$ & $13 \pm 3$ & $19 \pm 2$ & $22 \pm 4$ & $15 \pm 2$ \\
\hline & & 2 & $2,335-2,340$ & $8 \pm 1$ & $10 \pm 1$ & $11 \pm 2$ & $7 \pm 1$ \\
\hline & & 3 & $2,376-2,378$ & $11 \pm 3$ & $17 \pm 2$ & $15 \pm 2$ & $17 \pm 2$ \\
\hline & \multirow{3}{*}{ 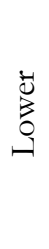 } & 4 & $2,400-2,410$ & $15 \pm 3$ & $15 \pm 2$ & $16 \pm 3$ & $14 \pm 2$ \\
\hline & & 5 & $2,425-2,430$ & $12 \pm 3$ & $14 \pm 2$ & $14 \pm 3$ & $14 \pm 2$ \\
\hline & & 6 & $2,438-2,440$ & $13 \pm 2$ & $17 \pm 2$ & $19 \pm 3$ & $14 \pm 2$ \\
\hline \multirow{2}{*}{  } & & 7 & $2,448-2,450$ & $15 \pm 3$ & $20 \pm 2$ & $16 \pm 4$ & $20 \pm 2$ \\
\hline & & 9 & $2,470-2,472$ & $26 \pm 4$ & $33 \pm 3$ & $34 \pm 5$ & $30 \pm 3$ \\
\hline
\end{tabular}

The maximum activity ratio of ${ }^{228} \mathrm{Ra} /{ }^{232} \mathrm{Th}$ is circa $1.5 \pm 0.6$ and refers to sample no. $3 .{ }^{228} \mathrm{Th} /{ }^{228} \mathrm{Ra}$ ratios range between $0.8 \pm 0.3$ and $1.2 \pm 0.4$. The average ${ }^{224} \mathrm{Ra} /{ }^{228} \mathrm{Th}$ ratio is $0.9 \pm 0.3$. With respect to measurement uncertainties, activity ratios are near unity and thus thorium decay series radionuclides have a state of secular equilibrium.

A detailed description about the measurement results presented in this section will be given in the following. Chapter 3 deepens the study on the reservoir rock material and the relating approach of reservoir identification of recent permeable fractures or, at least, previously permeable zones. Chapter 4 lists the findings of the Bruchsal fluid monitoring in detail and refers to the modelling part of Th-U series disequilibria for obtaining information about rates of water-rock interactions. Chapter 5 combines the results of both to an integrated approach into the characterization of the sitespecific fracture network.

\section{References}

Aquilina, L., Pauwels, H., Genter, A., Fouillac, C., 1997. Water-rock interaction processes in the Triassic sandstone and the granitic basement of the Rhine Graben: Geochemical investigations of a geothermal reservoir, in: 
Geochimica et Cosmochimica Acta, 61, S. 4281-4295.

Bateman, H. (1910). Solution of a system of differential equations occurring in the theory of radioactive transformations. Proceedings of the Cambridge Philosophical Society, 15, 423-427.

Condomines, M., Rihs, S., Lloret, E., Seidel, J.L., 2010. Determination of the four natural Ra isotopes in thermal waters by gamma-ray spectrometry. Appl. Radiat. Isot. 68, 384-391.

DEV D8, (1971). Deutsche Einheitsverfahren zur Wasser-, Abwasser- und Schlammuntersuchung - Anionen (Gruppe D) - Teil 8: Bestimmung von Hydrogenkarbonat (D 8).

DEV-B 1/2:1971, (1971). Deutsche Einheitsverfahren zur Wasser-, Abwasser- und Schlammuntersuchung - Prüfung von Geruch und Geschmack (B1/2).

DIN 38402-13:1985, (12 1985). Deutsche Einheitsverfahren zur Wasser-, Abwasser- und Schlammuntersuchung; Allgemeine Angaben (Gruppe A); Probenahme aus Grundwasserleitern (A 13).

DIN 38404:1976-12 (C4), (1976). Deutsche Einheitsverfahren zur Wasser-, Abwasser- und Schlammuntersuchung: Physikalische und physikalisch-chemische Kenngrößen (Gruppe C): Bestimmung der Temperatur (C4).

DIN 38404:1984-05 (C6), (1984). Deutsche Einheitsverfahren zur Wasser-. Abwasser- und Schlammuntersuchung Physikalische und physikalisch-chemische Kenngrößen (Gruppe C) - Bestimmung der Redox-Spannung (C6).

DIN 38405-21:1990-5 (D21), (1990). Deutsche Einheitsverfahren zur Wasser-, Abwasser- und Schlammuntersuchung - Anionen (Gruppe D) - Photometrische Bestimmung von gelöster Kieselsäure (D 21).

DIN 38405-27:2017-10 (D27), (2017). Deutsche Einheitsverfahren zur Wasser-, Abwasser- und Schlammuntersuchung - Annionen (Gruppe D) - Teil 27: Bestimmung von Sulfid durch Gasextration (D27).

DIN 38406-1:1983-5 (E1), (1983). Deutsche Einheitsverfahren zur Wasser-, Abwasser- und Schlammuntersuchung Kationen (Gruppe E) - Bestimmung von Eisen (E1).

DIN 38408:1984 (C9), (1984). Deutsche Einheitsverfahren zur Wasser-, Abwasser- und Schlammuntersuchung Physikalische und physikalisch-chemische Kenngrößen (Gruppe C) - Bestimmung der Dichte in wässriger Lösung (C9).

DIN 38408:1984 (G22), (1984). Deutsche Einheitsverfahren zur Wasser-, Abwasser- und Schlammuntersuchung Physikalische und physikalisch-chemische Kenngrößen (Gruppe G) - Bestimmung des in Wasser gelösten Sauerstoffs mittels membranbedeckter Sauerstoffsonden (G22).

DIN EN 1486:1997, (1997). Wasseranalytik - Anleitung zur Bestimmung des gesamten organischen Kohlenstoffs (TOC) und des gelösten organischen Kohlenstoffs (DOC).

DIN EN 27888:1993-11 (C8), (1993). Wasserbeschaffenheit - Bestimmung der elektrischen Leitfähigkeit (ISO 7888:1985). 
DIN EN I38404-2:1976-12 (C2), (1976). Deutsche Einheitsverfahren zur Wasser-, Abwasser- und Schlammuntersuchung - Physikalische und physikalisch-chemische Kenngrößen (Gruppe C) - Bestimmung der Trübung (C 2) (ISO 7027:1984).

DIN EN ISO 10304-1:2009-07, (2009). Wasserbeschaffenheit - Bestimmung von gelösten Anionen mittels Flüssigkeits-Ionenchromatographie - Teil 1: Bestimmung von Bromid, Chlorid, Fluorid, Nitrat, Nitrit, Phosphat und Sulfat (ISO 10304-1:2007).

DIN EN ISO 10523:2012-04 (C5), (2012). Wasserbeschaffenheit - Bestimmung des pH-Wertes (ISO 10523:2008).

DIN EN ISO 11885:2009-09 (E22), (2009). Wasserbeschaffenheit - Bestimmung von ausgewählten Elementen durch indukitv gekoppelte Plasma-Atom-Emissionsspektrometrie (ICP-OES) (ISO 1885:2007).

DIN EN ISO 17294-2:2017-01, (2017). Wasserbeschaffenheit - Anwendung der induktiv gekoppelten Plasma-Massenspektrometrie (ICP-MS) - Teil 2: Bestimmung von ausgewählten Elementen einschließlich Uran-Isotope (ISO 17294-2:2016).

DIN EN ISO 5667-1:2006, (2006). Wasserbeschaffenheit - Probenahme - Teil 1: Anleitung zur Erstellung von Probenahmeprogrammen und Probenahmetechniken.

DIN EN ISO 5667-3:2012, (2012). Wasserbeschaffenheit - Probenahme - Teil 3: Konservierung und Handhabung von Wasserproben (ISO 5667-3:2012).

DIN EN ISO 7027-2:2017, (2017). Wasserbeschaffenheit - Bestimmung der Trübung - Teil 2: Semi-quantitative Verfahren zur Beurteilung der Transparenz von Gewässern (ISO/DIS 7027-2:2017).

DIN EN ISO 7887:2011, (2011). Wasserbeschaffenheit - Untersuchung und Bestimmung der Färbung (ISO 7887:2011).

DIN EN ISO 8044:1999-11, (1999). Korrosion von Metallen und Legierungen - Grundbegriffe und Definitionen (ISO 8044:1999).

DIN ISO 11929:2011-01, (2011). Bestimmung der charakteristischen Grenzen (Erkennungsgrenze, Nachweisgrenze und Grenzen des Vertrauensbereichs) bei Messungen ionisierender Strahlung - Grundlagen und Anwendungen (ISO 11929:2010)

Eggeling, L., Herr, K., Goldberg, V., Siefert, D., Köhler, J., Kölbel, T., Reith, S., 2018. ANEMONA - Anlagenmonitoring als Schlüsseltechnologie für den erfolgreichen Betrieb von Geothermiekraftwerken in Deutschland. Teilprojekte: AP 10: Reservoircharakterisierung mittels Radionuklidanalytik, AP 11: Optimierung geothermischer Betriebsmonitoringsysteme am Beispiel von Bruchsal. Final report (published).

Fritz P., Eberwein P., Hackl S., Hornberger R., Schaumburg D., 1989. Geothermal project Bruchsal Phases 5 and 6, circulation and reinjection tests. Final report. Contract EEC n ${ }^{\circ}$ GE 265/85-DE.

Ivanovich, M., Murray, A., 1992. Spectroscopic methods, in: Ivanovich, M., Harmon, R.S. (Eds.), Uranium-series Disequilibrium: Applications to Earth, Marine, and Environmental Sciences. Clarendon Press, Oxford, $127-$ 
173.

Joachim, H., Koziorowski, G., Leiber, J., 1987. Geothermiebohrungen Bruchsal 1a und 2, in: Maus, H. (Ed.), Jahreshefte des Geologischen Landesamtes Baden-Württemberg 29. Herder, Freiburg i. Br., pp. 84-97.

LGRB, Federal State Office for Geology Resources and Mining, Freiburg Regional Board, 2020. LGRB-Kartenviewer. Retrieved March 11, .2020 from:https://maps.lgrb-bw.de/.

Rettenmaier, D., Gaucher, E., Ghergut, I., Huttenloch, P., Kohl, T., Meixner, J., Möllmann, G., Mundhenk, N., Rauppach, K., Rinke, M., Sauter, M., Seibt, A., Steger, H., Wolfgramm, M., Zorn, R., 2013. LOGRO Langzeitbetrieb und Optimierung eines Geothermiekraftwerks in einem geklüftet-porösen Reservoir im Oberrheingraben. Karlsruher Institut für Technologie (KIT), funding code 0325111 A, final report (unpublished).

Rytz, A., 1991. Recommended energy and intensity values of alpha particles from radioactive decay. Atomic Data and Nuclear Data Tables 47, 205-239.

Schötzig, U., Schrader, U., 1998. Halbwertszeiten und Photonen Emissionswahrscheinlichkeiten von häufig verwendeten Radionukliden, PTB-Ra-16/5.

Scheiber, J., Nitschke, F., Seibt, A., Genter, A., 2012. Geochemical and mineralogical monitoring of the geothermal power plant in Soultz-sous-Forêts (France). Proceedings of the 37th Workshop on Geothermal Reservoir Engineering, 2012, 1033-1042.

Pribnow, D., Schellschmidt, R., 2000. Thermal tracking of upper crustal fluid flow in the Rhine Graben. Geophysical Research Letters 27, 1957-1960.

Schneider, J., Eggeling, L., Hesshaus, A., 2014. Tiefengrundwassercharakteristik und hydrochemische Untersuchung. In: Bauer M., Freeden W., Jacobi H., Neu T. (eds) Handbuch Tiefe Geothermie. Springer Spektrum, Berlin, Heidelberg.

Vajda, N., Martin, P., Kim, C. K., 2012. Alpha spectrometry, in Handbook of Radioactivity Analysis, ed. M. F. L'Annunziata, Academic Press, 2012, ch. 6, pp. 363-422.

Wolfgramm. M., Seibt, A., 2008. Zusammensetzung von Tiefenwässern in Deutschland und ihre Relevanz für geothermische Anlagen. In: Proceed. Der Geothermiekongress 2008, Karlsruhe, Germany, 502-516.

Ziegler, P.A., 1992. European Cenozoic rift system. Tectonophysics 208, 91-111. 


\section{Chapter III}

\section{Identification of fractured zones in geothermal reservoirs in sedimentary basins: A radionuclide- based approach}

Lena Kölbel ${ }^{\mathrm{a},{ }^{*}}$, Thomas Kölbel ${ }^{\mathrm{b}}$, Martin Sauter ${ }^{\mathrm{a}}$, Thorsten Schäfer ${ }^{\mathrm{c}, \mathrm{d}}$, Dorothee Siefert ${ }^{\mathrm{b}}$, Bettina Wiegand $^{\mathrm{a}}$

Publication:

Kölbel, L., Kölbel, T., Sauter, M., Schäfer, T., Siefert, D., Wiegand, B, 2020. Identification of fractured zones in geothermal reservoirs in sedimentary basins: A radionuclide-based approach. Geothermics 85, 101764.

\footnotetext{
${ }^{a}$ University of Göttingen, Geosciences Center, Applied Geology, Goldschmidtstr. 3, 37077 Göttingen, Germany

b EnBW Energie Baden-Württemberg AG, Research and Innovation Department, Durlacher Allee 93, 76131 Karlsruhe, Germany

c Karlsruhe Institute of Technology (KIT), Institute for Nuclear Waste Disposal (INE), Hermann-von-Helmholtz Platz 1, 76344 Eggenstein-Leopoldshafen, Germany

d Friedrich Schiller University Jena, Institute of Geosciences, Applied Geology Burgweg 11, 07749 Jena, Germany

* Corresponding author. E-mail addresses: lena.koelbel@geo.uni-goettingen.de
} 


\section{Abstract}

A methodology for fracture zone identification in geothermal reservoirs was tested at the Bruchsal geothermal site, which is located close to the eastern main boundary fault of the Upper Rhine Graben. The site is explored and developed by a well doublet (GB1 and GB2). Fracture zones provide a substantial contribution to the effective hydraulic conductivity and pool the geothermal fluid circulation. In this context, water-rock interaction processes and the resulting changes in the composition of fluid and rock provide information on the type and the degree of fluid flow in the area of the fractured material. Thus, drill cuttings of the production well GB2 were analyzed with respect to their radionuclide signatures allowing conclusions on mechanisms and time-scale of the geochemical processes. The reservoir section at Bruchsal is defined by a radium anomaly (especially ${ }^{226} \mathrm{Ra}$ ) in deeper borehole sections corresponding to the Lower Buntsandstein and Permian Zechstein. The Bruchsal fluid, as well as other deep geothermal fluids in the Upper Rhine Valley, are characterized by radioactive disequilibria of radium isotopes $\left({ }^{226} \mathrm{Ra},{ }^{228} \mathrm{Ra},{ }^{224} \mathrm{Ra}\right)$ whose concentrations greatly exceed those of their thorium progenitors. It appears that radium is adsorbed onto hydrothermally altered minerals such as clay minerals and Fe-hydroxides or is co-precipitating with sulphate minerals such as barite. This leads to an average ${ }^{228} \mathrm{Ra} /{ }^{226} \mathrm{Ra}$ signature of 0.54 in the drill cuttings, in contrast to an expected value of 1.2 based on the average crustal $\mathrm{Th} / \mathrm{U}$ ratio of 3.8. For the Lower Buntsandstein and the Zechstein group, ${ }^{234} \mathrm{U} /{ }^{238} \mathrm{U}$ and ${ }^{230} \mathrm{Th} /{ }^{234} \mathrm{U}$ ratios are equal to unity, while ${ }^{226} \mathrm{Ra} /{ }^{230} \mathrm{Th}$ ratios range between 1.7 and 3.3. Radioactive disequilibrium was not observed for the thorium decay series. Using depth-related $\mathrm{Th} / \mathrm{Ra}$ and $\mathrm{Ra} / \mathrm{K}$ ratios, five fractured zones were identified within the Buntsandstein / Zechstein rock series, four of which were confirmed by conventional geophysical borehole logs. However, some issues remain unresolved such as the differentiation between active flow pathways and sealed fractures. 


\subsection{Introduction}

The detection of productive geothermal reservoir horizons and related pay zones is one of the major challenges in geothermal exploration and involve the methodology of various scientific disciplines such as geology, geochemistry as well as a range of geophysical techniques including geoelectrics, gravity, magnetics, and seismics (Ochieng, 2013; Ellis and Singer, 2007).

There are several techniques available, most of them are well logging based methods, e.g. resistivity, flow, or self-potential measurements (Ellis and Singer, 2007; Serra 1984). In practice, a single logging method is usually not sufficient for pay zone identification. Therefore, it is common to combine data from different logging techniques in an integrated evaluation procedure. These testing procedures have been shown to be robust and provide valuable information about the borehole itself as well as the related geology (Grant and Bixley, 2011).

On the other hand, logging tools can only be applied after drilling is completed or temporarily suspended. In addition, some logging methods require an open-hole section and potential borehole instabilities may limit their application. "Logging while drilling" devices might be an appropriate alternative (Bargach et al., 2000), but they have technical and financial limitations when applied under some adverse geothermal conditions (Habtemariam, 2012).

Radionuclide activities and their specific isotopic composition in cuttings provide an additional option to characterize geothermal reservoirs because the analysis of uranium and thorium decay series radionuclides can be used to study the hydrological regime within the rock. They can be analyzed and interpreted - with some acceptable delay - while drilling operations are in progress, or at a later stage in case other methods fail to provide the necessary information.

In the past, much focus has been placed on the uranium-thorium series disequilibrium in geothermal fluids because it provides site-specific information about radionuclide migration in the reservoir section (Ku et al., 1992, Lou et al., 2000, Zukin et al., 1987). Uranium and thorium decay series radionuclides are widespread in natural systems. In closed geological systems, more than $99 \%$ of the radioactive equilibrium will be reached after seven half-lives of the most long-lived radionuclides of the respective decay series. For instance, ${ }^{238} \mathrm{U}$ and its decay products reach equilibrium after $\sim 1.7$ million years (Porcelli et al., 2014). However, geothermal systems are not closed systems because of the geochemical interaction between the hot geothermal fluid with the reservoir rock and also with the meteoric system.

Tricca et al. (2001) describe these interaction processes as physico-chemical reactions taking place between three groups: the aqueous phase, the mineral grains and a reactive surface layer with a certain specific area and thickness. The transfer rate of a radionuclide from the rock material into the solution depends on: (a) the in-situ radioactive decay of its dissolved parent, (b) the desorption 
from the surface coating, (c) the alpha-recoil across the solid-liquid interface within a distance of several tens of nanometers, and (d) the dissolution of the aquifer solid. The removal of a radionuclide from the fluid depends on: (a) its radioactive decay in solution, (b) the adsorption onto the surface layer, and (c) the incorporation into precipitates (Ku et al., 1992).

Water-rock interaction involving radionuclides with variable chemical behavior and physical properties may result in radioactive disequilibria in fluids (Hussain and Krishnaswami, 1980). Therefore, deep geothermal fluids in the Upper Rhine Valley are highly concentrated with respect to radium isotopes $\left({ }^{226} \mathrm{Ra},{ }^{228} \mathrm{Ra},{ }^{224} \mathrm{Ra}\right.$ ) while their thorium progenitors $\left({ }^{230} \mathrm{Th},{ }^{232} \mathrm{Th},{ }^{228} \mathrm{Th}\right)$ present as $\mathrm{ThO}_{2}$, $\mathrm{ThO}_{2} \cdot x \mathrm{H}_{2} \mathrm{O}$ or $\mathrm{Th}(\mathrm{OH})_{4}$ have an expected low solubility under the geochemical conditions (Altmaier et al., 2005).

However, water-rock interaction processes do not only affect the radionuclide signature of the geothermal fluid, but also the associated solid. Primary minerals (mostly silicates) of the reservoir rock can be transformed into secondary minerals as a result of hydrothermal alteration (Browne, 1978). Boschmann (1986) postulated that Th/U ratios of newly formed hydrothermal minerals are substantially lower than the average crustal Th/U mean ratio of 3.8 published by Taylor and McLennan (1997). Moreover, Plyushchev and Ryabova (1974) investigated thorium-uranium concentrations in hydrothermal minerals from mineralized zones differing in formation characteristics and showed that these minerals have $\mathrm{Th} / \mathrm{U}$ ratios between $0.08-3.6$. The $\mathrm{Th} / \mathrm{U}$ mass ratio of 3.8 equals the ${ }^{232} \mathrm{Th} /{ }^{238} \mathrm{U}$ ratio of $1.2\left({ }^{232} \mathrm{Th} ; 1 \mathrm{ppm}=4.06 \mathrm{~Bq} \mathrm{~kg}^{-1} ;{ }^{238} \mathrm{U} ; 1 \mathrm{ppm}=12.35 \mathrm{~Bq} \mathrm{~kg}^{-1}\right.$ according to IAEA, 1989) and thus, radionuclide signatures of rocks can be assumed to reflect subsurface environmental conditions.

In this paper, a new approach for the identification of fractured permeable or at least previously permeable zones of active fluid circulation in boreholes is proposed based on activity ratios of thorium-uranium series radionuclides in rocks. This method has been tested at the Bruchsal geothermal site in Germany on a set of cuttings from the geothermal well GB2 and compared with the results of other well logs. The procedure and the results of this study are discussed below.

\subsection{Geological setting}

The Bruchsal site is located close to the eastern main boundary fault of the Upper Rhine Graben (Fig. 3.1). It is characterized by an NNE-SSW striking extension structure with a length of ca. 300 $\mathrm{km}$ and a width of approximately $40 \mathrm{~km}$. The deep Hercynian basement, composed of Paleozoic granites and gneisses, is overlain by sediments with a thickness of up to $4500 \mathrm{~m}$ (Ziegler, 1992). The heat flow rate in the graben ranges between $100-120 \mathrm{~mW} / \mathrm{m}^{2}$ (Pribnow and Schellschmidt, 2000). Borehole data of GB2 yielded a bottom hole temperature of $134.7^{\circ} \mathrm{C}$ at $2542 \mathrm{~m}$ depth. 


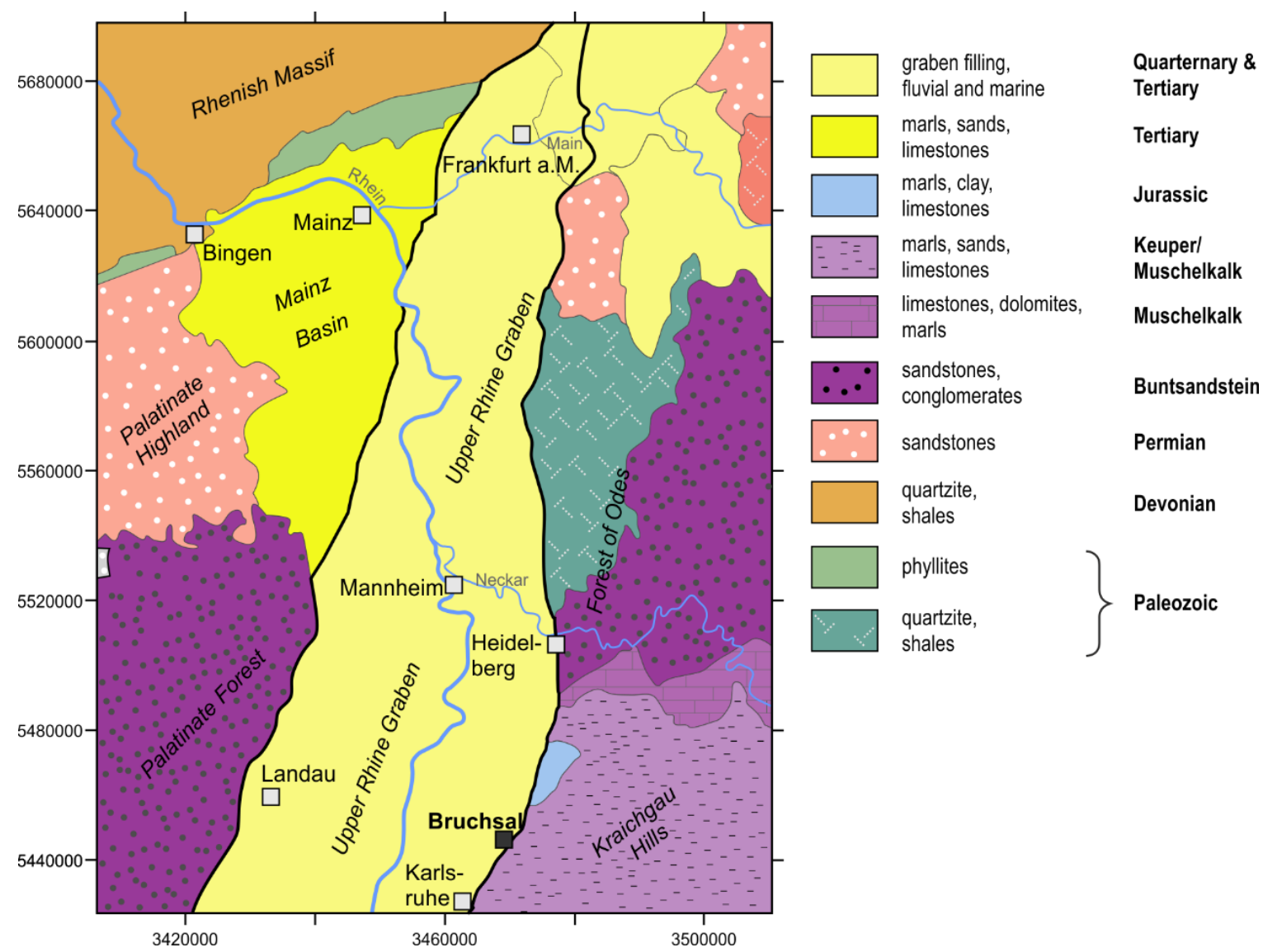

Fig. 3.1: Location of the Bruchsal geothermal site in the Upper Rhine Graben, Baden-Württemberg, Germany. Geology after GÜK300 (1989) and GÜK500 (1998).

This is equal to a geothermal gradient of $55 \mathrm{~K} \mathrm{~km}^{-1}$, which exceeds the average value for Central Europe of $30 \mathrm{~K} \mathrm{~km}^{-1}$.

The geothermal plant at Bruchsal comprises two boreholes with the injection well GB1 located 1.5 $\mathrm{km}$ north of the production well GB2 (Mergner et al., 2013). The geothermal reservoir units are located in the Middle and Lower Buntsandstein and the Permian Zechstein/Rotliegend formations identified by hydraulic tests. Partial mud losses were recorded at $2292 \mathrm{~m}$ (cased) and at $2435 \mathrm{~m}$ : total mud loss started at $2471 \mathrm{~m}$ (Joachim et al., 1987).

The reservoir section in the injection well GB1 differs in depth and thickness from that in GB2 because of tectonic displacements. Meixner et al. (2016) provided additional site-specific information about the local tectonic settings and geology. The Permian units are composed of fluvial sandstones ("Tigersandstein") and playa mudstones (Langenthal or "Bröckelschiefer") (Hagdorn and Nitsch, 2009). Buntsandstein sediments comprise mainly coarse to medium-grained clastic sandstones with a relatively low fraction of clay minerals, partly enriched with detritus of the crystalline basement (Geyer and Gwinner 2011). 
They are cut by large-scale listric normal faults dipping westward. A displacement of up to a few thousand meters of the rock series inside the graben relative to the same units in the neighboring shoulders in the Kraichgau region is documented by Ziegler (1992).

\subsection{Material and method}

Alpha- and gamma-ray measurements of the Bruchsal drill cuttings provide specific activities of the following radionuclides:

- Thorium decay series: ${ }^{232} \mathrm{Th},{ }^{228} \mathrm{Ra},{ }^{228} \mathrm{Th},{ }^{224} \mathrm{Ra}$

- Uranium decay series: ${ }^{238} \mathrm{U},{ }^{234} \mathrm{U},{ }^{230} \mathrm{Th},{ }^{226} \mathrm{Ra}$

A total of 98 cutting samples from Bruchsal GB2 representing mixtures of depth intervals from two to six meters were available for further laboratory analysis. The samples cover the section from the Keuper down to the Upper Zechstein formation in 1972 to $2470 \mathrm{~m}$ depth. The sampling depth is lag time corrected; the quantity of each individual sample is in the range 30 to $50 \mathrm{~g}$. Cross contamination by drilling mud can be excluded. After drilling all cuttings were stored by the Geological Survey of the state of Baden Wuerttemberg.

These drill cuttings were first characterized by laboratory gamma spectrometry. The gamma-ray measurements were performed with a p-type HPGe detector with a relative efficiency of $30 \%$ (GC3018-CP-5SL-P). Each sample was measured for 20 hours. All measurements and analyses described in the following paragraphs were carried out by EnKK Philippsburg laboratory (Germany).

In Table 3.1 the investigated radionuclides and the applied analytical methods are listed. ${ }^{226} \mathrm{Ra}\left(\mathrm{t}_{1 / 2}\right.$ $=1600 \mathrm{a})$ was analyzed based on its short-lived granddaughters ${ }^{214} \mathrm{~Pb}\left(\mathrm{t}_{1 / 2}=27 \mathrm{~min}\right)$ and ${ }^{214} \mathrm{Bi}\left(\mathrm{t}_{1 / 2}\right.$ $=20 \mathrm{~min}$ ). Prior to the measurements, the samples were sealed to minimize radon losses. ${ }^{222} \mathrm{Rn}$ is the decay product of ${ }^{226} \mathrm{Ra}$ and has a half-life of 3.8 days. After an ingrowth period of 20 days, the specific activity of ${ }^{222} \mathrm{Rn}$ grew due to the radioactive decay of its parent and secular equilibrium with ${ }^{226} \mathrm{Ra}$ was achieved to $97 \%$. Secular equilibrium is defined by the daughter activity being equal to the activity of its parent.

This type of radioactive equilibrium typically occurs when the half-life of the parent is much larger (by several orders of magnitude) than the daughter's half-life. Compared to the timescale being considered, the decay rate of ${ }^{226} \mathrm{Ra}$ (and so the supply rate of its daughter) stays constant. Similarly, ${ }^{228} \mathrm{Ra}$ activity was determined by the specific activity of its daughter ${ }^{228} \mathrm{Ac}\left(\mathrm{t}_{1 / 2}=6.2 \mathrm{~h}\right)$. Here, secular equilibrium was achieved after a time delay of 60 hours between sampling and measurement. 
Table 3.1: Overview of the radionuclides investigated and the applied examination methods. Data about $\alpha$-particle energy E $\alpha$ and emission probability $\mathrm{P} \alpha$ are reported by Rytz (1991) while data about gamma-ray energies E $\gamma$ and emission probabilities P $\gamma$ are from Schötzig and Schrader (1998) *Possible interference from Bi-211 (E $\gamma=351.1 \mathrm{keV}$ / $\mathrm{P} \gamma=12.9 \%)$.

\begin{tabular}{|c|c|c|c|c|c|c|}
\hline $\begin{array}{l}\text { Decay } \\
\text { series }\end{array}$ & $\begin{array}{l}\text { Radionuclide } \\
\text { investigated }\end{array}$ & Half-life & $\begin{array}{l}\text { Analytical } \\
\text { methods }\end{array}$ & $\begin{array}{l}\text { Measured ra- } \\
\text { dionuclides }\end{array}$ & $\begin{array}{l}\text { Energy } \\
\mathrm{E}_{\alpha, \gamma}(\mathrm{keV})\end{array}$ & $\begin{array}{l}\text { Probability } \\
\mathrm{P}_{\alpha, \gamma}(\%)\end{array}$ \\
\hline \multirow{8}{*}{$\begin{array}{l}\text { Uranium } \\
\text { series }\end{array}$} & $\mathrm{U}-238$ & $4.468 \times 10^{9} \mathrm{y}$ & $\alpha$ & $\mathrm{U}-238$ & 4,198.0; 4,151.0 & $77.0 ; 23.0$ \\
\hline & $\mathrm{U}-234$ & $2.455 \times 10^{5} \mathrm{y}$ & $\alpha$ & $\mathrm{U}-234$ & $4,774.6 ; 4,722.4$ & $72.5 ; 27.5$ \\
\hline & Th-230 & $7,538 \times 10^{4} \mathrm{y}$ & $\alpha$ & Th-230 & $4,687.0 ; 4,620.5$ & $76.3 ; 23.4$ \\
\hline & Ra-226 & $1,600 \mathrm{y}$ & $\alpha$ & $226-\mathrm{Ra}$ & $4,784.3 ; 4,601.0$ & $94.5 ; 5.5$ \\
\hline & & & $\gamma$ & $\mathrm{Pb}-214$ & $295.2 ; 242.0$ & 18.2; 7.1; \\
\hline & & & & & $(351.9)^{*}$ & 35.1 \\
\hline & & & $v^{\prime}$ & Bi-214 & $609.3 ; 1,120.3$ & \\
\hline & & & 1 & & $1,764.5$ & 15.1 \\
\hline \multirow{5}{*}{$\begin{array}{l}\text { Thorium } \\
\text { series }\end{array}$} & Th-232 & $1,405 \times 10^{10} \mathrm{y}$ & $\alpha$ & Th-232 & $4,013.0 ; 3,950.0$ & $77.0 ; 23.0$ \\
\hline & $\mathrm{Ra}-228$ & $5.75 \mathrm{y}$ & $\gamma$ & Ac-228 & $911.2 ; 969.0$ & $25.8 ; 15.8$ \\
\hline & & & & & 338.3 & 11.3 \\
\hline & Th-228 & $1.913 \mathrm{y}$ & $\alpha$ & Th-228 & $5,423.2 ; 5,340.4$ & $73.4 ; 26.6$ \\
\hline & Ra-224 & $3.66 \mathrm{~d}$ & $\gamma$ & $\mathrm{Pb}-212$ & 238.6 & 43.3 \\
\hline
\end{tabular}

${ }^{224} \mathrm{Ra}$ activity was calculated from the measured activity of its daughter ${ }^{212} \mathrm{~Pb}$. The half-live of the parent ${ }^{224} \mathrm{Ra}\left(\mathrm{t}_{1 / 2}=3.66 \mathrm{~d}\right)$ is also longer than that of its daughter ${ }^{212} \mathrm{~Pb}\left(\mathrm{t}_{1 / 2}=10.6 \mathrm{~h}\right)$, but of a similar magnitude. This condition is defined as transient equilibrium. Here, the daughter activity exceeds the activity of its parent which leads to a ${ }^{212} \mathrm{~Pb} /{ }^{224} \mathrm{Ra}$ ratio of ca. 1.14 following a rest period of 100 hours after sampling.

Since uranium $\left({ }^{238} \mathrm{U},{ }^{234} \mathrm{U}\right)$ and thorium $\left({ }^{232} \mathrm{Th},{ }^{230} \mathrm{Th},{ }^{228} \mathrm{Th}\right)$ isotopes are alpha emitting radionuclides 
nine samples were additionally investigated by alpha spectrometry. Therefore, solid samples were sequentially leached with $\mathrm{HCl}, \mathrm{HF}$ and aqua regia. Any solid residue remaining was completely digested in $\mathrm{HF}$ /aqua regia in a PFA container at $120^{\circ} \mathrm{C}$. Once total dissolution was achieved, appropriate spikes were added. Alpha spectrometric measurements of ${ }^{238} \mathrm{U},{ }^{234} \mathrm{U},{ }^{232} \mathrm{Th},{ }^{230} \mathrm{Th},{ }^{228} \mathrm{Th}$ and ${ }^{226} \mathrm{Ra}$ by isotope dilution as outlined in Knight et al. (2004) were performed.

To compare both spectrometric methods, ${ }^{226} \mathrm{Ra}$ was measured by alpha and gamma spectrometry. Fig. 3.2 shows that both methods lead to similar results and confirm our approach of combining both techniques. In this way, it is possible to almost completely determine the radionuclide composition of the uranium and thorium decay series.



Fig. 3.2: Specific activity of ${ }^{226} \mathrm{Ra}(\mathrm{Bq} / \mathrm{kg})$ in the GB2 drill cuttings analyzed by alpha and gamma spectrometry. The solid line indicates a 1:1 correlation. Error bars represent uncertainties due to counting statistics $( \pm 1 \sigma)$.

Assuming that hydrothermal alteration processes resulting from the interaction between circulating geothermal fluid and the host rock characterize geothermal reservoirs, a petrographic alteration study was performed. Hydrothermal alteration minerals, such as clay minerals, were analyzed under the microscope. Therefore, thin sections of $30 \mu \mathrm{m}$ thickness were prepared from the GB2 drill cuttings and investigated by optical polarizing microscopy. Some of the thin sections were subsequently coated with carbon and examined by scanning electron microscope (FEI QUANTA 650). The latter was used for morphological studies as well as for determining the elemental composition semi-quantitatively by energy dispersive X-ray fluorescence (EDX).

Radionuclide signatures associated with secondary alteration processes in the rock samples allow the identification of possible water inflow zones. In order to validate these results, geophysical 
borehole logs of GB2 were used.

The fluid circulation and physico-chemical processes in geothermal systems affect the physical properties of the subsurface causing measurable anomalies in a number of state variables. Therefore, physical parameters such as temperature, electrical conductivity, permeability of the rock material and others, are key indicators for the detection of geothermal reservoir sections and related reservoir horizons.

For deeper borehole sections of GB2 the following logs are available: natural gamma ray (NGR), deep and shallow formation resistivity tool (DLL), caliper log (CAL) and temperature log (TEMP). The gamma-ray log quantifies the natural radioactivity emanating from the rock formations. Natural gammy-ray logs are often used for the identification of lithological units. In sandstone reservoirs, their main purpose is to obtain an indication of the clay content. The dual laterolog tool is used in boreholes to determine formation resistivity and it provides two resistivity measurements at different depths of investigation at the same time. In fractured rocks, large separations are observed between shallow (LLs) and deep (LLd) laterolog curves. Caliper logs measure the average diameter of the borehole by using several spring-loaded arms. Significant borehole breakouts on the caliper log may indicate a fractured zone. Temperature logs provide continuous temperature profiles in a borehole. They may detect thermal anomalies produced by temperature contrasts between the drilling fluid and the formation or convective heat/mass flow in the relevant section, respectively. Further details regarding the methodological techniques can be found in Ellis and Singer (2007).

\subsection{Results}

\subsubsection{Alteration petrography}

The study of alteration characteristics is focused on the reservoir section within the Middle and Lower Buntsandstein formation as well as Zechstein rocks i.e. depths between $2178-2472 \mathrm{~m}$ in the production well GB2. In this section, the cuttings consist of sandstones, siltstones, and mudstones.

The sandstone fragments are mainly composed of quartz and K-feldspar. Cements vary greatly between siliceous, clayey and carbonatic composition depending on the diagenetic history. Mudstones are primarily composed of quartz, Fe-hydroxides, feldspars, and monazite detritus. The matrix comprises illitic clay minerals.

Quartr is present in all samples as sand- and silt-sized grains with a predominantly pink coloring. From a depth of $2378 \mathrm{~m}$, a frequent occurrence of polycrystalline quartz is observed. Furthermore, some quartz grains are coated by iron hydroxides or calcite. 
Feldspar grains have been detected in almost all samples. They are present at a concentration less than $20 \%$ and are mainly formed by K-feldspars. With only a few exceptions, plagioclase appears to be absent. White decomposed feldspars are predominant at depths between 2387 and $2472 \mathrm{~m}$.

Heavy minerals comprise single grains of rutile, monazite, apatite, and zirconia. Their occurrence is often accompanied by relatively high contents of Th and rare earth elements such as Ce, La, and $\mathrm{Nd}$.

The petrographic observations indicate transformation and replacement of primary minerals by secondary mineral phases by hydrothermal processes. An alteration zone can be defined for the depth interval 2387 - $2472 \mathrm{~m}$ indicated by illite that dominates the clay fraction of most samples. Illite has most likely been formed by the decomposition of feldspars. It is present as authigenic sandstone cement and arranged along grain boundaries.

An authigenic pyrite framboids are limited to the depth range 2267 - $2342 \mathrm{~m}$, which corresponds stratigraphically to the Middle Buntsandstein. Barite has been detected in some samples of the Middle and Lower Buntsandstein as well as in the Zechstein group. Barite mineralization resulted from hydrothermal fluids circulating along grain boundaries and micro-fissures. Iron bydroxide was mainly found in sandstone fragments between depths of 2387 and $2458 \mathrm{~m}$. They are partly present as limonite coatings around quartz grains.

Carbonates have been detected in many samples, mostly present as $\mathrm{Mg}-\mathrm{Fe}-\mathrm{Mn}$-Ca-bearing carbonatic cements in sandstone fragments. The corresponding minerals are siderite $\left(\mathrm{FeCO}_{3}\right)$, ankerite $\left(\mathrm{CaFe}\left[\mathrm{CO}_{3}\right]_{2}\right)$, and dolomite $\left(\mathrm{CaMg}\left[\mathrm{CO}_{3}\right]_{2}\right)$. In addition, a high proportion of fossiliferous calcite fragments were found in drill cuttings from 2330 to $2343 \mathrm{~m}$ depth. These fragments were probably caving material from overlying strata. Shell fragments were also detected in samples from 2360 to $2387 \mathrm{~m}$ depth. Over a depth interval from 2400 to $2472 \mathrm{~m}$ the samples are characterized by calcite fragments without any organic material that may constitute filling of veins and micro cracks. In the drilling logs, carbonaceous fracture filling was frequently reported for this particular depth interval.

\subsubsection{Results from spectrometric measurements}

The ${ }^{238} \mathrm{U}$ activity in the GB2 cuttings of the reservoir section ranges between 10 and $22 \mathrm{~Bq} / \mathrm{kg}$ whereas the specific activity of ${ }^{232} \mathrm{Th}$ ranges between 8 and $25 \mathrm{~Bq} / \mathrm{kg}$. These values are relatively low, but typical for sandstones (Adams and Weaver, 1958). The ${ }^{232} \mathrm{Th} /{ }^{238} \mathrm{U}$ ratios calculated from the measured activities of ${ }^{238} \mathrm{U}$ and ${ }^{232} \mathrm{Th}$ vary between $0.8-1.3$ and they are in good agreement with the mean ratio in the continental crust of 1.2 (Porcelli et al., 2014).

\subsubsection{Signature of radium isotopes}

Fig. 3.3 illustrates the specific activity of ${ }^{226} \mathrm{Ra}$ versus ${ }^{228} \mathrm{R}$ a measured in the GB2 drill cuttings of 
the Buntsandstein and Zechstein section. The specific activities of ${ }^{226} \mathrm{Ra}$ range between 8 and 44 $\mathrm{Bq} / \mathrm{kg}$ and between 9 and $39 \mathrm{~Bq} / \mathrm{kg}$ for ${ }^{228} \mathrm{Ra}$, respectively. Individual radium signatures mark the single geological units.

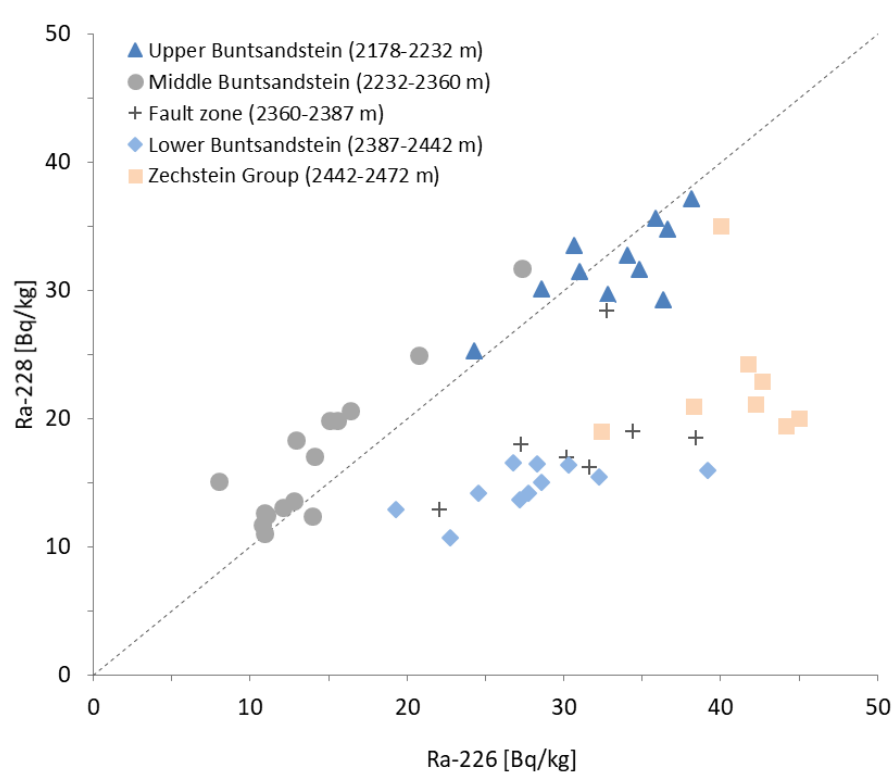

Fig. 3.3: Relationship between ${ }^{228} \mathrm{Ra}(\mathrm{Bq} / \mathrm{kg})$ and ${ }^{226} \mathrm{Ra}(\mathrm{Bq} / \mathrm{kg})$. The dashed line displays a ${ }^{228} \mathrm{Ra} /{ }^{226} \mathrm{Ra}$ ratio $=1$. Cuttings of the deeper borehole sections are characterized by ${ }^{228} \mathrm{Ra} /{ }^{226} \mathrm{Ra}$ ratios $<1$.

Assuming radioactive equilibrium within the respective decay series, the activity ratio of ${ }^{228} \mathrm{Ra} /{ }^{226} \mathrm{Ra}$ should equal the ratio of their ${ }^{232} \mathrm{Th} /{ }^{238} \mathrm{U}$ parents that is about 1.2 (plotted as dashed line in Fig. 3.3). ${ }^{228} \mathrm{Ra} /{ }^{226} \mathrm{Ra}$ ratios of the Upper and Middle Buntsandstein samples vary by \pm 0.2 from the expected value of about 1 . All other samples show a ${ }^{228} \mathrm{Ra} /{ }^{226} \mathrm{Ra}$ ratio significantly lower than 1 caused by a rising ${ }^{226} \mathrm{Ra}$ activity with increasing depth. Consequently, the ${ }^{226} \mathrm{Ra}$ activity is highest in the Zechstein samples $\left(34-46 \mathrm{~Bq} / \mathrm{kg}\right.$ ). The mean ${ }^{228} \mathrm{Ra} /{ }^{226} \mathrm{Ra}$ activity ratio of the Lower Buntsandstein and Zechstein is approximately 0.54 . This is in good agreement with the observed ${ }^{228} \mathrm{Ra} /{ }^{226} \mathrm{Ra}$ signature of 0.55 in the Bruchsal geothermal fluid (Eggeling et al., 2013).

\subsubsection{Uranium series disequilibrium}

Plots of the ${ }^{234} \mathrm{U} /{ }^{238} \mathrm{U},{ }^{230} \mathrm{Th} /{ }^{234} \mathrm{U}$ and ${ }^{226} \mathrm{Ra} /{ }^{230} \mathrm{Th}$ specific activity ratios $\left[\mathrm{Bq} \mathrm{kg}{ }^{-1} / \mathrm{Bq} \mathrm{kg}^{-1}\right]$ are presented in Fig. 3.4. Samples of the Middle Buntsandstein show secular equilibrium within a range of one standard deviation $(1 \sigma)$ analytical error.

For the Lower Buntsandstein and Zechstein groups, ${ }^{234} \mathrm{U} /{ }^{238} \mathrm{U}$ and ${ }^{230} \mathrm{Th} /{ }^{234} \mathrm{U}$ activity ratios are also equal to unity while ${ }^{226} \mathrm{Ra} /{ }^{230} \mathrm{Th}$ activity ratios higher than unity indicate general disequilibria with values ranging from a minimum of 1.7 to a maximum of 3.3 . Here, the ${ }^{226} \mathrm{Ra}$ activity exceeds 

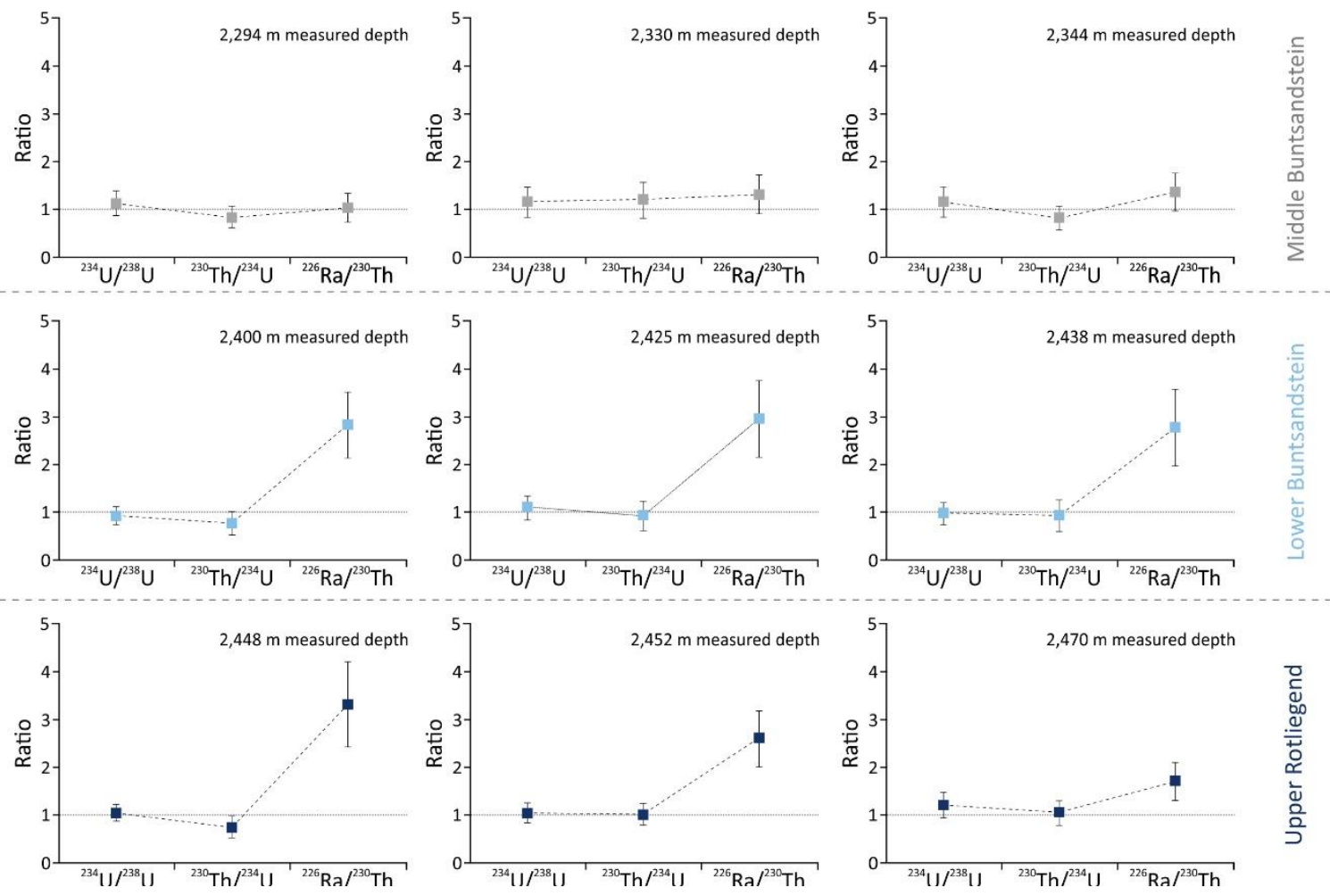

Fig. 3.4: Radionuclide disequilibrium within the reservoir section of GB2: cuttings of the Lower Buntsandstein and the older Permian Zechstein are significantly enriched with respect to ${ }^{226} \mathrm{Ra}$. Error bars represent uncertainties due to counting statistics $( \pm 1 \sigma)$.

the specific activity of ${ }^{238} \mathrm{U}$ and its decay products ${ }^{234} \mathrm{U}$ and ${ }^{230} \mathrm{Th}$ by a factor of 2 to 3.

${ }^{230} \mathrm{Th} /{ }^{234} \mathrm{U}$ ratios near unity suggest that uranium migration has not taken place relative to thorium for the last $10^{5}-10^{6}$ years. In contrast, the high ${ }^{226} \mathrm{Ra} /{ }^{230} \mathrm{Th}$ activity ratios in the deeper rock samples show that radium has been highly concentrated in the solid phase relative to thorium. This result is surprising in that radioactive equilibrium was expected for ${ }^{238} \mathrm{U}$ and its decay products due to the geological age of the rocks of about 250 million years.

\subsubsection{Thorium decay series radionuclides}

Table 3.2 summarizes the results of ${ }^{232} \mathrm{Th},{ }^{228} \mathrm{Ra},{ }^{228} \mathrm{Th}$, and ${ }^{224} \mathrm{Ra}$ activities measured in the GB2 cuttings. In contrast to the uranium decay series, there is no radioactive disequilibrium observed for the thorium decay series radionuclides. There are some slight variations between the specific activities of thorium and radium isotopes, but this can be explained by measurement uncertainties.

Buntsandstein samples are characterized by uniform activities of the thorium decay series radionuclides (Table 3.2). The average ${ }^{232} \mathrm{Th}$-activity in drill cutting of the Middle and Lower Buntsandstein is about $12 \pm 2 \mathrm{~Bq} / \mathrm{kg}$. In contrast, Zechstein samples show substantially higher activities of ${ }^{232} \mathrm{Th}$, 
up to $26 \pm 4 \mathrm{~Bq} / \mathrm{kg}$.

Activity ratios are close to unity. This applies to all activity ratios $\left[\mathrm{Bq} \mathrm{kg}^{-1} / \mathrm{Bq} \mathrm{kg}^{-1}\right]$, the ${ }^{228} \mathrm{Ra}^{232} \mathrm{Th}$, ${ }^{228} \mathrm{Th} /{ }^{228} \mathrm{Ra}$, and the ${ }^{224} \mathrm{Ra} /{ }^{228} \mathrm{Th}$ activity ratio. The maximum activity ratio of ${ }^{228} \mathrm{Ra} /{ }^{232} \mathrm{Th}$ is circa $1.5 \pm 0.6$ and refers to sample no. $3 .{ }^{228} \mathrm{Th} /{ }^{228} \mathrm{Ra}$ ratios range between $0.8 \pm 0.3$ and $1.2 \pm 0.4$. The average ${ }^{224} \mathrm{Ra} /{ }^{228} \mathrm{Th}$ ratio is $0.9 \pm 0.3$. With respect to measurement uncertainties, activity ratios are near unity and thus thorium decay series radionuclides have a state of secular equilibrium.

Table 3.2: Thorium decay series: Specific activity of thorium $\left({ }^{232} \mathrm{Th},{ }^{228} \mathrm{Th}\right)$ and radium isotopes $\left({ }^{228} \mathrm{Ra},{ }^{224} \mathrm{Ra}\right)$ in the GB2 drill cuttings. The analytical error is ca. $\pm 1 \sigma$.

\begin{tabular}{|c|c|c|c|c|c|c|c|}
\hline \multicolumn{2}{|c|}{ Geology } & Sample & Depth period & ${ }^{232} \mathrm{Th}$ & ${ }^{228} \mathrm{Ra}$ & ${ }^{228} \mathrm{Th}$ & ${ }^{224} \mathrm{Ra}$ \\
\hline \multirow{6}{*}{ 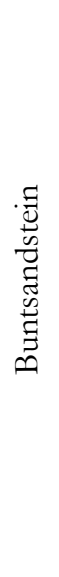 } & \multirow{3}{*}{$\frac{\ddot{\theta}}{\vec{\nabla}}$} & 1 & $2,330-2,335$ & $13 \pm 3$ & $19 \pm 2$ & $22 \pm 4$ & $15 \pm 2$ \\
\hline & & 2 & $2,335-2,340$ & $8 \pm 1$ & $10 \pm 1$ & $11 \pm 2$ & $7 \pm 1$ \\
\hline & & 3 & $2,376-2,378$ & $11 \pm 3$ & $17 \pm 2$ & $15 \pm 2$ & $17 \pm 2$ \\
\hline & \multirow{3}{*}{ 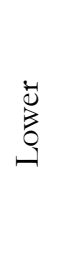 } & 4 & $2,400-2,410$ & $15 \pm 3$ & $15 \pm 2$ & $16 \pm 3$ & $14 \pm 2$ \\
\hline & & 5 & $2,425-2,430$ & $12 \pm 3$ & $14 \pm 2$ & $14 \pm 3$ & $14 \pm 2$ \\
\hline & & 6 & $2,438-2,440$ & $13 \pm 2$ & $17 \pm 2$ & $19 \pm 3$ & $14 \pm 2$ \\
\hline \multirow{3}{*}{ 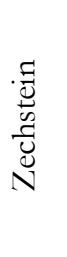 } & & 7 & $2,448-2,450$ & $15 \pm 3$ & $20 \pm 2$ & $16 \pm 4$ & $20 \pm 2$ \\
\hline & & 8 & $2,452-2,454$ & $20 \pm 3$ & $23 \pm 1$ & $24 \pm 3$ & $23 \pm 2$ \\
\hline & & 9 & $2,470-2,472$ & $26 \pm 4$ & $33 \pm 3$ & $34 \pm 5$ & $30 \pm 3$ \\
\hline
\end{tabular}

\subsection{Discussion}

A ${ }^{226} \mathrm{Ra}$-anomaly within the Lower Buntsandstein and Zechstein section of the production well GB2 was identified from alpha- and gamma-ray measurements. Based on the age of the PermoTriassic reservoir rock, radioactive equilibrium between radionuclides of the respective natural decay series can be expected. The enrichment of ${ }^{226} \mathrm{Ra}$ in the reservoir rock is probably caused by water-rock interaction between the geothermal fluid and fractured rock material. 
The saline Bruchsal fluid is highly concentrated in $\mathrm{Na}^{+}$and $\mathrm{Cl}^{-}$, and other alkali and earth alkali ions, with up to $127 \mathrm{~g} / \mathrm{l}$ of total dissolved solids (Eggeling et al., 2011). Radium isotopes $\left({ }^{224} \mathrm{Ra}\right.$, ${ }^{228} \mathrm{Ra},{ }^{226} \mathrm{Ra}$ ) are several orders of magnitude in excess of their parent nuclides $\left({ }^{230} \mathrm{Th}\right.$ for ${ }^{226} \mathrm{Ra},{ }^{232} \mathrm{Th}$ for ${ }^{228} \mathrm{Ra}$, ${ }^{228} \mathrm{Th}$ for ${ }^{224} \mathrm{Ra}$ ). For example, the specific activity of ${ }^{226} \mathrm{Ra}$ in the geothermal fluid is up to $30 \mathrm{~Bq} / \mathrm{kg}$ (Eggeling et al., 2013).

It is assumed that the enrichment of ${ }^{226} \mathrm{Ra}$ in the reservoir rock is produced by a removal of ${ }^{226} \mathrm{Ra}$ from the geothermal fluid. Generally, radium removal from the aqueous phase may take place by cation exchange, sorption processes, and/or co-precipitation. Radium is one of the most strongly adsorbed earth alkali ions during ion exchange on clay minerals under certain conditions (Porcelli et al., 2014). Furthermore, oxide minerals have a high potential for radium adsorption. There are several studies on radium sorption involving manganese oxides and different hydroxides available (Ames et al., 1983, Benes et al., 1984; Valentine at al., 1990). However, the results of these studies indicate that the adsorption behavior of radium depends on several factors such as temperature, Eh-pH-conditions, and the presence of other alkaline earth elements. Due to high temperatures and low $\mathrm{pH}$-values however, scavenging of radium by sorption processes might play a minor role in a geothermal environment, both decreasing the adsorption capacity with respect to radium ( $\mathrm{Zu}$ kin et al., 1987). Furthermore, Tanner (1964) proposed that during cation exchange Ra-adsorption may be reduced because of the competition between radium and other alkaline earth metals for sorption sites resulting in an enrichment of radium in saline waters due to Ra-displacement from the rock surface by other cations with higher affinity for the exchange sites.

At Bruchsal the reservoir section consists of quartz-dominated sandstones $\left(\mathrm{SiO}_{2}\right.$ concentration up to 80 vol.- $\%$ ). According to Carroll (1959), the cation exchange capacity (CEC) of quartz is about $0.6 \mathrm{meq} / 100 \mathrm{~g}$ and therefore, has to be judged rather poor. This is also the case for feldspar (CEC $=1.0-2.0 \mathrm{meq} / 100 \mathrm{~g}$ ) which is strongly decomposed in the reservoir section and is mainly represented by K-feldspar with a maximum proportion of 20 vol.- $\%$. In contrast, CEC of clay minerals can be 100 times higher (Carroll, 1959), and therefore, clays seem to have the greatest effect on the cation exchange capacity of the bulk rock. Based on the petrographic studies, clay minerals belong to the illite group which corresponds to a cation exchange capacity of 10 - $40 \mathrm{meq} / 100 \mathrm{~g}$ (Carroll, 1959). Cation exchange capacity of the entire rock is estimated at $1.50-2.60 \mathrm{meq} / 100 \mathrm{~g}$ depending on the proportion of clay minerals, which reaches up to 8 vol.- $\%$.

The quantity of radium isotopes adsorbed on the rock surface was determined by means of MIN3P, a multicomponent reactive transport model (Mayer at al., 2002). For calculations, an average CEC value of $2.00 \mathrm{meq} / 100 \mathrm{~g}$ was chosen. Thermodynamic data for cation exchange were taken from the PHREEQC2-database (Appelo and Postma, 1993) With respect to the chemical composition of the Bruchsal fluid (Eggeling et al., 2013); radium competes with $\mathrm{Na}, \mathrm{K}, \mathrm{Mg}$, Sr, Ba and Cs for cation exchange sites. The results show that the sorbed radium species vary between $10^{-17}$ and $10^{-}$ 
${ }^{12} \mathrm{meq} / 100 \mathrm{~g}$. As can be seen from Table 3.3, the proportion of radium adsorbed to the rock surface depends on the concentration of the particular radium isotope in the fluid. Raising the fluid concentration of radium by a factor of three $\left({ }^{226} \mathrm{Ra} \approx 30 \mathrm{~Bq} / \mathrm{L} ;{ }^{224} \mathrm{Ra} \approx 10 \mathrm{~Bq} / \mathrm{L}\right)$ increases the amount of adsorbed radium by six orders of magnitude. Although adsorption processes take place, it becomes obvious from the low values that ion exchange only constitutes a small part.

The partition between the dissolved and adsorbed components through chemical exchanges, such as cation exchange and surface complexation, can be described by the distribution coefficient, $K_{d}$, defined as $C_{d} / C$ (Krishnaswami et al., 1982), where $C_{d}$ is the concentration of the adsorbed radionuclides in aquifer solids in atoms per mass and $C$ is concentration of dissolved radionuclides in atoms per fluid-volume. The distribution coefficients for radium, expressed in units of volume per mass, are displayed in Table 3.3.

Table 3.3: Comparison of radium dissolved and adsorbed in the geothermal system in Bruchsal as well as the resulting distribution coefficient $K_{d}$.

\begin{tabular}{cccc}
\hline $\begin{array}{c}\text { Isotopes of } \\
\text { radium }\end{array}$ & $\begin{array}{c}\text { Activity of dissolved Ra } \\
(\mathrm{Bq} / \mathrm{L})\end{array}$ & $\begin{array}{c}\text { Adsorbed Ra proportion } \\
(\mathrm{meq} / 100 \mathrm{~g})\end{array}$ & $\begin{array}{c}\text { Ra distribution coefficient } \\
K_{d}(\mathrm{~mL} / \mathrm{g})\end{array}$ \\
\hline${ }^{226} \mathrm{Ra}$ & 29.9 & $1.2 \times 10^{-11}$ & 0.016 \\
${ }^{228} \mathrm{Ra}$ & 16.2 & $1.9 \times 10^{-14}$ & 0.014 \\
${ }^{224} \mathrm{Ra}$ & 10.6 & $3.9 \times 10^{-17}$ & 0.014 \\
\hline
\end{tabular}

Retardation factor due to sorption of radium isotopes can be determined by the following equation (Ku et al., 1992):

$$
R_{f}=1+K=1+\frac{C_{a}}{C}
$$

where

K dimensionless distribution coefficient;

$C_{a} \quad$ concentration of an adsorbed radionuclide, atoms per equivalent fluid-volume;

C concentration of a dissolved radionuclide, atoms fluid-volume ${ }^{-1}$.

According to Krishnaswami et al. (1982), the relation between $K$ and $K_{d}$ can be expressed as

$$
K=K_{d}\left[\frac{\rho_{s}(1-\phi)}{\phi}\right]
$$


where

$\rho_{S} \quad$ density of aquifer solids, mass solid volume ${ }^{-1}$;

$\phi \quad$ porosity of the aquifer, dimensionless.

Since the porosity of the reservoir is about $5 \%$ and rock density is about $2.7 \mathrm{~g} / \mathrm{cm}^{3}$, the dimensionless distribution coefficient $K$ ranges between 0.7 for the short-lived ${ }^{224} \mathrm{Ra}$ and ${ }^{228} \mathrm{Ra}$ and 0.8 for the long-lived ${ }^{226} \mathrm{Ra}$. From equation (3.1), the maximum retardation factor is 1.8 , which suggests a minor retardation due to sorption. This underlines the statement above that radium sorption due to cation exchange is not the decisive reason for the accumulation of ${ }^{226} \mathrm{Ra}$ observed in the reservoir section.

Therefore, the accumulation of ${ }^{226} \mathrm{Ra}$ observed in the Bruchsal drill cuttings might be caused primarily by co-precipitation, a process which affects mainly long-lived radionuclides such as ${ }^{226} \mathrm{Ra}$. Radium isotopes differ strongly in their half-lives from a few days $\left({ }^{224} \mathrm{Ra}=3.66 \mathrm{~d}\right)$ to several hundred years $\left({ }^{226} \mathrm{Ra}=1600 \mathrm{a}\right) .{ }^{228} \mathrm{Ra}$ is also one of the short-lived radionuclides with a half-life of 5.8 years. Short-lived radionuclides are characterized by relatively large decay constants. In general, the shorter the half-life $\left(t_{1 / 2}\right)$, the larger the decay constant $(\lambda)$. Thus, for the short-lived ${ }^{224} \mathrm{Ra}$ and ${ }^{228} \mathrm{Ra}$, decay constants are larger than $3.82 \times 10^{-9} \mathrm{~s}^{-1}$. In this case, the precipitation rate constant $k_{p}$ has to be unrealistically large to be significant as proposed by Porcelli (2008). However, the situation is different with ${ }^{226} \mathrm{Ra}$ with a decay constant of ca. $1.373 \times 10^{-11} \mathrm{~s}^{-1}$ and therefore, enough time is available for precipitation processes. Thus, the restriction to long-lived radionuclides could be a possible explanation for only ${ }^{226} \mathrm{Ra}$ enrichment in the cuttings while the short-lived radium isotopes $\left({ }^{224} \mathrm{Ra},{ }^{228} \mathrm{Ra}\right)$ are in a secular equilibrium.

Langmuir and Melchior (1985) found that the concentrations of dissolved radium in some deep brines in north Texas were likely to be controlled by co-precipitation in sulphate minerals due to the high concentrations of sulphate and earth alkali ions. This observation is confirmed by current studies dealing with the formation of Ra-bearing barite in German geothermal sites (Heberling at al., 2017; Haas-Nüesch at al., 2018).

Barite is a typical sulphate mineral incorporating radium in solid solution as $[\mathrm{Ba}, \mathrm{Ra}] \mathrm{SO}_{4}$. Both alkaline earth ions possess equal ionic charge and have similar length ionic radii (radium $=1.52 \AA$, barium $=1.35 \AA$ according to Shannon, 1976). Earth alkali ions can also be incorporated in carbonate minerals. However, radium levels observed in calcite are much lower than those observed in barite because of the larger difference in ionic radii between radium and calcium ions compared to that of radium and barium (Bove and Felmlee, 1982).

The findings of our petrographic study defined an alteration zone between 2387 and $2472 \mathrm{~m}$ depth characterized by the frequent occurrence of barite, carbonates, and iron hydroxide as well as illite 
formed by feldspar decomposition. Thus, from a mineralogical point of view, both co-precipitation in sulphate minerals/carbonates as well as adsorption on clay minerals/metal hydroxides are potentially responsible for radium scavenging from the geothermal fluid.

Nevertheless, it might be reasonable to assume that the ${ }^{226} \mathrm{Ra}$-anomaly is mainly caused by barite precipitation because of the preferred enrichment of the long-lived ${ }^{226} \mathrm{Ra}$. Relating to the geothermal system at Bruchsal, it appears that the formation of barite is linked to a local replacement of the carbonatic cements indicating hydrothermal activity.

Hydrothermal alteration processes result from the interaction of the geothermal fluid with the associated solid in fluid circulation zones. Therefore, alteration minerals are linked to reservoir sections with an increased hydraulic permeability. Fractured zones provide a substantial portion of the permeability due to the low porosity of the Bruchsal sandstone reservoir rock.

Assuming that the formation of barite is controlled by hydrothermal activities occurring mainly in water circulation horizons, productive zones might therefore be identified by elevated levels of ${ }^{226} \mathrm{Ra}$ co-precipitating primarily in sulphate minerals such as barite.

Fracture zones with increased ${ }^{226} \mathrm{Ra}$ activity can be displayed by depth-related $\mathrm{Th} / \mathrm{Ra}$ und $\mathrm{Ra} / \mathrm{K}$ ratio. Therefore, the measured activities of ${ }^{226} \mathrm{Ra},{ }^{232} \mathrm{Th}$, and ${ }^{40} \mathrm{~K}$ (all in $\mathrm{Bq} / \mathrm{kg}$ ) were converted to radium and thorium concentrations in $\mathrm{ppm}$ and potassium in percent, respectively. According to the International Atomic Energy Agency, 1 ppm ${ }^{226} \mathrm{Ra}$ equals $11.1 \mathrm{~Bq} / \mathrm{kg}, 1 \mathrm{ppm}{ }^{232} \mathrm{Th}$ equals 4.06 $\mathrm{Bq} / \mathrm{kg}$, and $1 \%$ of ${ }^{40} \mathrm{~K}$ equals $313 \mathrm{~Bq} / \mathrm{kg}$ (IAEA, 1989).

The Th/Ra and $\mathrm{Ra} / \mathrm{K}$ curves are inversely proportional to each other and consequently changes in radium content result in their inclines in opposite directions. Significant changes in radium concentration indicated by changes in these ratios are marked in blue in Fig. 3.5. To confirm the presence of the fracture zones identified, the results were compared to conventional logging data from the production well GB2.

Fig. 3.5 presents ratios $\mathrm{Th} / \mathrm{Ra}$ and $\mathrm{Ra} / \mathrm{K}$ as well as natural gamma ray, caliper, resistivity, and temperature logs for the GB2 well. The evaluation of the logging data is focused on the depth interval between 2285 and $2540 \mathrm{~m}$, which corresponds to the slotted liner section of GB2. Five possible water inflow zones have been identified by the comparison of the $\mathrm{Th} / \mathrm{Ra}$ and $\mathrm{Ra} / \mathrm{K}$ ratios.

Zone 1. The first radium anomaly in the slotted liner interval is detected between depths $2330 \mathrm{~m}$ and $2340 \mathrm{~m}$ (start of the 7" liner section). However, geophysical borehole logs do not show any indication of fractured rock in this interval.

Zone 2. The second interval $(2360-2364 \mathrm{~m})$ is characterized by a strong increase in the gamma-ray activity. Fossil bearing limestone fragments indicate a fault zone. The caliper log shows significant borehole breakouts, while no anomaly is observed in the temperature log. In our interpretation, this observation may indicate a fractured zone with a very low hydraulic conductivity. 




Fig. 3.5: Stratigraphical sequence of Buntsandstein and Zechstein in the open-hole section of GB2. Radium anomalies indicate five fractured zones (blue boxes) which were compared to results obtained by conventional logging tools. Temperature logs cover the time between the completion of drilling and flow tests (oldest left side, youngest right side).

Zone 3. The third interval, between 2378 and $2385 \mathrm{~m}$, is still a part of the above fault zone characterized by a large amount of carbonates. The caliper log exhibits a low anomaly at a constant borehole diameter. However, the most prominent feature is observed in the resistivity log. A sharp decrease in resistivity of at least one order of magnitude and a negative separation of LLs and LLd might indicate a fractured zone. The gamma-ray log presents a slight decrease in activity probably caused by the increasing quartz proportion visible in the cuttings.

Zone 4. The fourth zone is detected at the depth interval $2430-2435 \mathrm{~m}$. No anomaly is apparent in the gamma-ray log. However, there is a general increase in the gamma-ray signal for the complete depth profile because of an increase in clay mineral content as indicated by the microscope cutting analysis. This depth interval stratigraphically corresponds to the Lower Buntsandstein section and is defined as an alteration zone evidenced by the petrographic study.

Again, a significant reduction in the specific resistivity signal is found in Fig. 3.5. The separation of the results of the deep and shallow resistivity measurements exceeds the section indicated by the 
$\mathrm{Th} / \mathrm{Ra}$ and $\mathrm{Ra} / \mathrm{K}$ curves by a factor of 2 . Borehole breakouts with an intensity comparable to that of zone 3 are observed in the caliper log.

Zone 5. The last zone is the most prominent and covers the depth interval $2442-2472 \mathrm{~m}$. This section corresponds stratigraphically to the Permian Zechstein. The cuttings contain sandstone and large amounts of mudstone fragments believed to be responsible for the positive peaks in the gamma-ray log. Furthermore, decomposed feldspars, calcite and iron hydroxide were observed under the microscope indicating an active alteration horizon.

A significant anomaly is reflected by the changes in temperature and the temperature gradient, respectively. Five temperature logs were performed within one month. The logging campaign started two days after drilling was completed. Thus, the data correspond to the initially disrupted temperature profile when cool drilling mud penetrated the formation. Usually, this effect ceases with time. Hydraulically active fissures are also indicated by significant borehole breakouts observed in the caliper log. As shown in the resistivity log, there are several peaks in the low resistivity section.

Table 3.4 summarizes the integrated geophysical log interpretation with respect to the five zones detected by ${ }^{226} \mathrm{Ra}$-anomalies. With the exception of zone 1 , every other zone can be identified by one or more conventional logs as a possibly productive horizon. However, there is no individual $\log$ that can identify productive zones on its own. It is also interesting to note that the high-resolution temperature log only shows an anomaly in the highly conductive fractured zone 5 of the Permian Zechstein (2442 - 2472 m). Furthermore, it appears that the discrimination between hydraulically active and non-active fractures cannot be achieved by the radionuclide signatures in the host rock alone because fractures can also be sealed by precipitates of hydrothermal minerals.

Table 3.4: Synopsis of geophysical borehole logs of the five possible fracture zones defined by radionuclide signatures. A cross indicates anomalies recognized in the particular borehole log. Abbreviations: $\mathrm{GR}=$ gamma ray log; $\mathrm{CAL}=$ caliper log; tempGRAD = temperature gradient; $\mathrm{DLL}_{-}=$dual laterolog for formation resistivity; TEMP = temperature $\log$.

\begin{tabular}{|c|c|c|c|c|c|c|}
\hline & $\begin{array}{c}\text { GR } \\
{[\mathrm{API}]}\end{array}$ & CAL [inch] & $\begin{array}{c}\text { tempGRAD } \\
{[\mathrm{K} / \mathrm{km}]}\end{array}$ & DLL [ohm.m] & TEMP $\left[{ }^{\circ} \mathrm{C}\right]$ & Mud losses \\
\hline \multicolumn{7}{|l|}{ Zone 1} \\
\hline Zone 2 & $\mathrm{x}$ & $\mathrm{x}$ & & & & \\
\hline Zone 3 & & $\mathrm{x}$ & & $\mathrm{x}$ & & \\
\hline Zone 4 & & $\mathrm{x}$ & & $\mathrm{x}$ & & x (partly) \\
\hline Zone 5 & $\mathrm{x}$ & $\mathrm{x}$ & $\mathrm{x}$ & $\mathrm{x}$ & $\mathrm{x}$ & $\mathrm{x}$ (total) \\
\hline
\end{tabular}


This is obvious in zone 2 where ${ }^{226} \mathrm{Ra}$ activities and the caliper log indicate a fractured zone, which is, however, not confirmed in the temperature log. Therefore, data evaluation will be best when several well logging methods are combined in a joint evaluation.

\subsection{Conclusions}

In this study, an alternative method for the characterization of geothermal reservoirs is presented. Special attention is paid to the interpretation of radionuclide signatures in fractured zones which channel the geothermal fluid in the reservoir. In the Bruchsal reservoir fractured and hydrothermally altered horizons are associated with a preferential accumulation of ${ }^{226} \mathrm{Ra}$ in the solid phase. It was found that the ${ }^{228} \mathrm{Ra} /{ }^{226} \mathrm{Ra}$ activity ratio is significantly lower in samples from deeper borehole sections than the expected ${ }^{228} \mathrm{Ra} /{ }^{226} \mathrm{Ra}$ ratio of 1.2. Thus, it seems that the geothermal reservoir is limited to the depth interval 2330 - 2472 m, which corresponds to the Lower Buntsandstein and Zechstein units.

The enrichment of ${ }^{226} \mathrm{R}$ a causes a disruption of the secular equilibrium of the uranium decay series. Within this decay series, the specific activity of ${ }^{226} \mathrm{Ra}$ exceeds the ${ }^{230} \mathrm{Th}$ activity by a factor of two to three, while the ${ }^{230} \mathrm{Th} /{ }^{234} \mathrm{U}$ and ${ }^{234} \mathrm{U} /{ }^{238} \mathrm{U}$ ratios are equal to unity, within an analytical error of one standard deviation, indicating that uranium has not been transported relative to thorium for the last $10^{5}-10^{6}$ years.

Therefore, the accumulation of ${ }^{226} \mathrm{Ra}$ in the rock material is presumably the result of water-rock interaction between the hot geothermal fluid and the associated solid. Co-precipitation of ${ }^{226} \mathrm{Ra}$ in sulphate minerals such as barite is considered to be the most likely reason for the observed ${ }^{226} \mathrm{Ra}$ anomaly because co-precipitation is favored by long-lived radionuclides. Adsorption onto clay minerals and metal hydroxides may play a secondary role. However, it needs further site-specific investigations concerning the chemical behavior of radium under different environmental conditions (redox, temperature, salinity) to gain a detailed process understanding.

Within the open-hole section of the production well GB2, five possible fractured zones were identified by the evaluation of $\mathrm{Th} / \mathrm{Ra}$ and $\mathrm{Ra} / \mathrm{K}$ ratios which are sensitive to changes in the ${ }^{226} \mathrm{Ra}$ concentration. Four of the zones were detected by different logging tools such as natural gammaray, resistivity, temperature, and caliper logs. However, there are indications that some zones are sealed by the precipitation of secondary minerals. In fact, this is a limitation for our method because it is not possible to distinguish between recent and paleo-fractures solely by radionuclide signatures. Nevertheless, the investigation of radionuclide distributions in natural reservoirs could be regarded as a supplementary method to define productive geothermal horizons. 


\section{Acknowledgements}

This research was carried out within the framework of the ANEMONA project, funded by the German Federal Ministry for Economic Affairs and Energy (BMWi). The authors would like to thank the geoscientific department of the University of Tuebingen for the great support for the petrographic analysis. We are also grateful to the laboratories of EnKK (Philippsburg) and IAF (Dresden) that performed the alpha- and gamma-ray measurements.

\section{References}

Adams, J.A. and Weaver, C.B., 1958: Thorium-uranium ratios as indicators of sedimentary processes: Example of concept of geochemical fades. - American Association of Petroleum Geologists Bulletin, 42: 387-430.

Altmaier, M., Neck, V., Muller, R. \& Fanghanel, T, 2005. Solubility of ThO2 center dot xH(2)O(am) in carbonate solution and the formation of ternary Th(IV) hydroxide-carbonate complexes. Radiochim. Acta 93, 83-92.

Ames, L.L., McGarrah, J.E., Walker, A.B., Salter, P.F., 1983. Uranium and radium sorption on amorphous ferric oxyhydoxide. Geochemical Geology 40, 135-148.

Appelo, C. A. J., Postma, D., 1993. Geochemistry, Groundwater and Pollution. Balkema, Rotterdam.

Bargach, S., Falconer, I., Maeso, C., Codazzi, D., Hodenfield, K., Ford, G., Hartner, J., Grether, B., Rohler, H., 2000. Real-Time LWD: Logging for drilling. Oilfield Review, autumn 2000, 58-78.

Benes, P., Strejc, P., Lukavec, Z., 1984. Interaction of radium with fresh-water sediments and their mineral components, 1 - ferric hydroxide and quartz. Journal of Radioanalytical and Nuclear Chemistry 82, 275-285.

Boschmann, W., 1986: Uran und Helium in Erzmineralen und die Frage über ihre Datierbarkeit. Heidelberger Geowissenschaftliche Abhandlungen 6, 1-234.

Bove, D., Felmlee, J.K., 1982. Mineralogy and autoradiography of selected mineral-spring precipitates in the Western United States. Open-File Report of the United States Department of Interior Geological Survey.

Browne, P.R.L., 1978. Hydrothermal alteration in active geothermal fields. Annual Review Earth and Planetary Sciences $6,229-250$.

Carroll, D., 1959. Ion exchange in clays and other minerals. Geological Society of America Bulletin 70, 749-780.

Eggeling, L., Genter, A., Kölbel, T., Münch, W., 2013. Impact of natural radionuclides on geothermal exploitation in the Upper Rhine Graben. Geothermics 47, 80-88.

Eggeling, L., Kölbel, T., Schlagermann, P., Münch, W., 2011. Geothermische Stromerzeugung in Deutschland. CIT 83, 1834-1844. 
Ellis, D.V., Singer, J.M., 2007. Well Logging for Earth Scientists, Springer, Dordrecht.

Geyer, O.F., Gwinner, M., 2011. Geologie von Baden-Württemberg, Schweizerbart, Stuttgart.

Grant, M.A., Bixley, P.F., 2011. Geothermal Reservoir Engineering, Elsevier, Oxford.

GÜK300, 1989. Geological overview of Hesse (1:300.000). Hessian regional authorities for ground research, Wiesbaden.

GÜK500, 1998. Geological overview of Baden-Württemberg (1:500.000). State Office of Geology, Raw Materials and Mining, Freiburg i.Br.

Haas-Nüesch, R., Heberling, F., Schild, D., Rothe, J., Dardenne, K., Jähnichen, S., Eiche, E., Marquardt, C., Metz, V., Schäfer, T., 2018: Mineralogical characterization of scalings formed in geothermal sites in the Upper Rhine Graben before and after the application of sulfate inhibitors. In: Geothermics 71, 264-273.

Habtemariam, B.W., 2012. Main technical issues regarding problems when drilling geothermal wells, UNU-GTP, Iceland, Report 36/2012, 915-952.

Hagdorn, H., Nitsch, E., 2009. 6th International Triassic Field Workshop: Triassic of Southwest Germany, Conference paper, Tübingen.

Heberling, F., Schild, D., Degering, D., Schäfer, T., 2017. How well suited are current thermodynamic models to predict or interpret the composition of $(\mathrm{Ba}, \mathrm{Sr}) \mathrm{SO} 4$ solid-solutions in geothermal scalings? In: Geotherm Energy 5 (1).

Hussain, N., Krishnaswami, S., 1980. U-238 series radioactive disequilibrium in groundwater: implications to the origin of U-234 excess and fate of reactive pollutants. Geochimica et Cosmochimica Acta 44, 287-1291.

International Atomic Energy Agency IAEA, 1989. Construction and use of calibration facilities for radiometric field equipment. Technical reports series No.309, Vienna.

Joachim, H., Koziorowski, G., Leiber, J., 1987. Geothermiebohrungen Bruchsal 1a und 2, in: Maus, H. (Ed.), Jahreshefte des Geologischen Landesamtes Baden-Württemberg 29. Herder, Freiburg i. Br., pp. 84-97.

Knight, A.W., Eithreim, E.S., Nelson, A.W., Nelson, S., Schultz, M.K., 2004. A simple-rapid method to separate uranium, thorium, and protactinium for U-series age-dating of materials. J Environ. Radioact. 134, 66-74.

Krishnaswami, S., Graustein, W.C., Turekian, K.K., Dowd, J.F., 1982. Radium, thorium and radioactive lead isotopes in groundwaters: Applications to the in situ determination of adsorption-desorption rate constants and retardation factors. Water Resour. Res. 18 (6), 1633-1675.

Ku, T.-L., Lou, S., Leslie, B.W., Hammond, D.E., 1992. Decay-series disequilibria applied to the study of water-rock interaction and geothermal systems, in: Ivanovich, M., Harmon, R.S. (Eds.), Uranium-series Disequilibrium: Applications to Earth, Marine, and Environmental Sciences. Clarendon Press, Oxford, 631-668.

Langmuir, D., Melchior, D., 1985. The geochemistry of Ca, Sr, Ba, and Ra sulfates in some deep brines from the 
Palo Duro Basin, Texas. Geochim. Cosmochim. Acta 49, 2423-2432.

Lou, S., Ku, T.-L., Roback, R., Murrell, M., McLing, T.L., 2000. Decay-series disequilibrium of in-situ, long-term radionuclide transport in water-rock systems. Proceedings on Scientific Basis for Nuclear Waste Management XXIII, Material Research Society.

Mayer, K.U., Frind, E.O., Blowes, D.W., 2002. Multicomponent reactive transport modeling in variably saturated porous media using a generalized formulation for kinetically controlled reactions. Water Resour. Res. 38, 1174.

Meixner, J., Schill, E., Grimmer, J.C., Gaucher, E., Kohl, T., Klingler, P., 2016. Structural control of geothermal reservoirs in extensional tectonic settings: An example from the Upper Rhine Graben. Journal of Structural Geology 82, 1-15.

Mergner, H., Koelbel, T., Schlagermann, P., 2013. Geothermal power generation - First operation experiences and performance analysis of the Kalina Plant in Bruchsal. PowerGen Proceedings, London, 2013.

Ochieng, L., 2013. Overview of geothermal surface exploration methods. Short Course VIII on Exploration for Geothermal Resources, Kenya, Oct. 31 - Nov. 22, 2013.

Plyushchev, Y.V., Ryabova, L.A., 1974. Accumulation levels of U and Th in hydrothermal minerals. Geochem. Inter. $11,820-830$.

Porcelli, D., 2008. Investigating groundwater processes using U- and Th-series nuclides, in: Baxter, M.S. (Ed.), Radioactivity in the Environment 13. Elsevier, Amsterdam, 105-153.

Porcelli, D., Kim, C.-K., Martin, P., Moore, W.S., Phaneuf, M., 2014. Properties of radium, in: IAEA (Ed.), the environmental behavior of radium (revised edition), technical report series no. 476, Vienna, 6-32.

Pribnow, D., Schellschmidt, R., 2000. Thermal tracking of upper crustal fluid flow in the Rhine Graben. Geophysical Research Letters 27, 1957-1960.

Rytz, A., 1991. Recommended energy and intensity values of alpha particles from radioactive decay. Atomic Data and Nuclear Data Tables 47, 205-239.

Schötzig, U., Schrader, U., 1998. Halbwertszeiten und Photonen Emissionswahrscheinlichkeiten von häufig verwendeten Radionukliden, PTB-Ra-16/5.

Serra, O., 1984. Fundamentals of well-log interpretation: The acquisition of logging data. Elsevier, Amsterdam.

Shannon, R.D., 1976. Revised effective ionic radii and systematic studies of interatomic distances in halides and chalcogenides. Acta Crystallographica 32, 751-767.

Tanner, A.B., 1964. Radon migration in the ground: a review, in: Adams, J.A.S and Lowder, W.M. (Eds.), the natural radiation environment, Univ. Chicago Press, Chicago, 161-190.

Taylor, S.R., McLennan, S.M., 1997. The origin and evolution of the Earth's continental crust. Journal of Australian 
Geology and Geophysics 17, 55-62.

Tricca, A., Wasserburg, G.J., Porcelli, D., Baskaran, M., 2001. The transport of U- and Th-series nuclides in a sandy unconfined aquifer. Geochim. Cosmochim. Acta 65, 1187-1210.

Valentine, R.L., Spangler, K.M., Meyer, J., 1990. Removing radium by adding preformed hydrous manganese oxides. Journal of the American Water Works Association 82, 66-71.

Ziegler, P.A., 1992. European Cenozoic rift system. Tectonophysics 208, 91-111.

Zukin, J.G., Hammond, D.E., Ku, T.-L., Elders, W.A., 1987. Uranium-Thorium series radionuclides in brines and reservoir rocks from two deep geothermal boreholes in the Salton Sea geothermal field, southeastern California. Geochim. Cosmochim. Acta 51, 2719-2731. 


\section{Chapter IV}

\section{Water-rock interactions in the Bruchsal geothermal system by $\mathrm{U}$-Th series radionuclides}

Lena Kölbel a, ${ }^{\text {, }}$, Thomas Kölbel ${ }^{b}$, Ullrich Maier ${ }^{a}$, Martin Sauter ${ }^{a}$, Thorsten Schäfer ${ }^{c}$, Bettina Wiegand $^{\mathrm{a}}$

Manuscript:

Kölbel, L., Kölbel, T., Maier, U., Sauter, M., Schäfer, T., Wiegand, B, 2020. Water-rock interactions in the Bruchsal geothermal system by U-Th series radionuclides. Submitted to Geothermal Energy on July 03, 2020 (minor revision - August 11, 2020).

a University of Göttingen, Geosciences Center, Applied Geology, Goldschmidtstr. 3, 37077 Göttingen, Germany

b EnBW Energie Baden-Württemberg AG, Research and Innovation Department, Durlacher Allee 93, 76131 Karlsruhe, Germany

c Friedrich Schiller University Jena, Institute of Geosciences, Applied Geology Burgweg 11, 07749 Jena, Germany

* Corresponding author. E-mail addresses: lena.koelbel@geo.uni-goettingen.de 


\section{Abstract}

Uranium and thorium decay series disequilibria in deep geothermal brines are a result of waterrock interaction processes. The migratory behavior of radionuclides provides valuable site-specific information and can therefore be an important tool for reservoir characterization and sustainable management of geothermal sites.

In this study, we present data from long-term monitoring of naturally occurring ${ }^{238} \mathrm{U},{ }^{232} \mathrm{Th}$ and ${ }^{235} \mathrm{U}$ series radionuclides analyzed in brine samples collected from the Permo-Triassic sedimentary reservoir rock at the Bruchsal geothermal site (SW Germany). The results show that radionuclides of the elements radium $\left({ }^{226} \mathrm{Ra},{ }^{228} \mathrm{Ra},{ }^{224} \mathrm{Ra},{ }^{223} \mathrm{Ra}\right)$, radon $\left({ }^{222} \mathrm{Rn}\right)$, and lead $\left({ }^{210} \mathrm{~Pb},{ }^{212} \mathrm{~Pb}\right)$ are rather soluble in brine, while isotopes of uranium $\left({ }^{238} \mathrm{U},{ }^{234} \mathrm{U},{ }^{235} \mathrm{U}\right)$, thorium $\left({ }^{232} \mathrm{Th},{ }^{228} \mathrm{Th},{ }^{230} \mathrm{Th}\right.$ ), polonium $\left({ }^{210} \mathrm{Po}\right)$, and actinium $\left({ }^{227} \mathrm{Ac},{ }^{228} \mathrm{Ac}\right)$ have low solubilities and are mostly immobile. Activities of radium isotopes in the geothermal brine exceed those of their thorium progenitors (average ${ }^{226} \mathrm{Ra}=$ $29.9 \mathrm{~Bq} \mathrm{~kg}^{-1}$, about $10^{3}$ times that of its ${ }^{230} \mathrm{Th}$ parent).

Modelling the observed disequilibria allows the following conclusion on water-rock interaction processes: (1) Supply from alpha recoil depends on isotope half-life because it is limited by the rate of diffusion through microfractures causing isotopic fractionation. (2) Radium retardation due to adsorption is low $\left({ }^{226} \mathrm{Ra} /{ }^{222} \mathrm{Rn}=1.3\right)$ resulting in adsorption-desorption rate constants in the order of $10^{-10} \mathrm{~s}^{-1}$ for $k_{1}$ and $10^{-9}$ for $k_{2}$. (3) Scavenging of ${ }^{226} \mathrm{Ra}$ from brine can best be explained by coprecipitation with barite resulting in an observed ${ }^{226} \mathrm{Ra}$ anomaly in the solids of the reservoir section. The precipitation rate constant amounts to ca. $3.4 \times 10^{-8} \mathrm{~s}^{-1}$ corresponding to a mean removal time of radium from brine by mineral precipitation to approximately one year. 


\subsection{Introduction}

In geothermal brines activities of naturally occurring radionuclides are controlled by a number of processes including radioactive decay and production, recoil supply, adsorption-desorption, and precipitation-dissolution.

The natural decay chains of ${ }^{238} \mathrm{U},{ }^{232} \mathrm{Th}$ and ${ }^{235} \mathrm{U}$ comprise elements with different hydrogeochemical properties and with more than one isotope (Fig. 4.1). While in closed systems all daughter nuclides will achieve secular radioactive equilibrium with respect to their parent nuclides (daughter/parent activity ratio becomes unity), deep geothermal brines interact with solid phases with whom they come into contact. In consequence of these water-rock interactions, an elemental fractionation occurs resulting in a state of disequilibria (Osmond and Cowart, 1992). Such radioactive disequilibria were found in deep geothermal brines in the Upper Rhine Graben (URG). Here, radium isotopes $\left({ }^{228} \mathrm{Ra},{ }^{226} \mathrm{Ra},{ }^{224} \mathrm{Ra},{ }^{223} \mathrm{Ra}\right.$ ) have activities far exceeding those of their thorium progenitors (Eggeling et al., 2013). Radioactive disequilibria caused by the preferred solution of radium were also documented for deep geothermal brines in the Salton Sea Geothermal field (Zukin et al., 1987). Previous studies have shown that radium concentrations are often high in saline waters (Kraemer and Reid, 1984; Dickson, 1985) and geothermal brines (Hammond et al., 1988; Rihs and Condomines, 2002; Condomines et al., 2012), but rather low in low-temperature, low-salinity groundwaters (Krishnaswami et al., 1982; Luo et al., 2000; Porcelli, 2014).

uranium-radium decay series

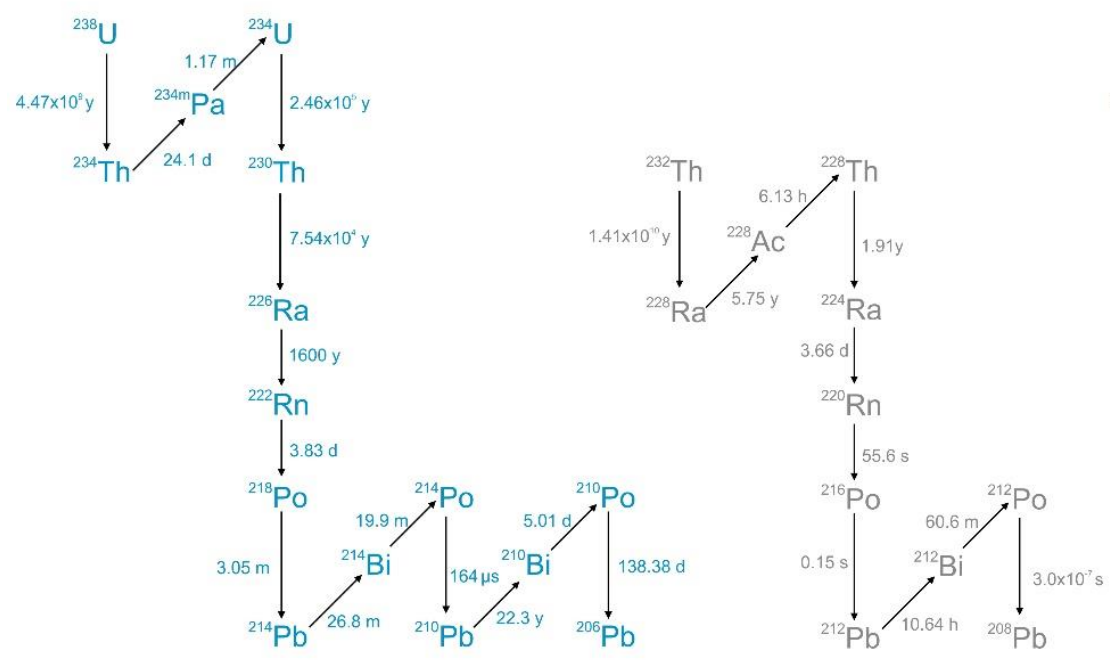

\section{actinium decay series}

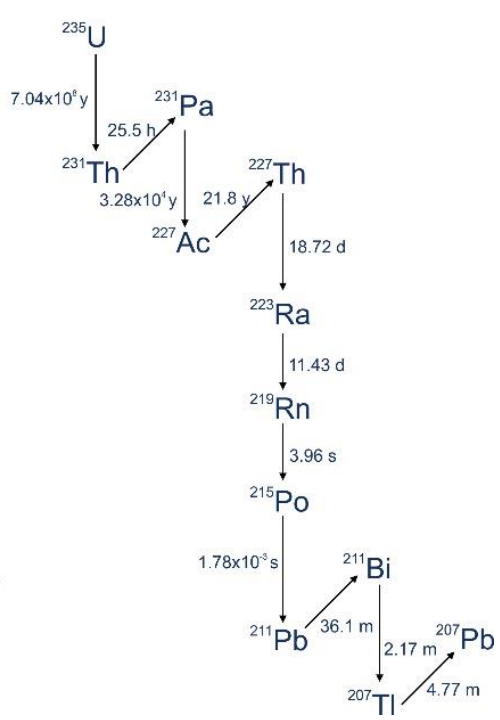

Fig. 4.1: Uranium-radium, thorium and actinium decay series. Vertical arrows indicate alpha decay; diagonal arrows indicate beta decay. For radionuclides with more than one decay mode, only the most frequently occurring is given. 
Modelling of these disequilibria provides information about the respective water-rock interaction processes controlling radionuclide supply into and scavenging from solution, respectively. This information is very useful to investigate the long-term migratory behavior of uranium and thorium series radionuclides which is not only an important issue for nuclear waste disposal, but also for geothermal sites in terms of handling radionuclide-bearing precipitated minerals (scales) in surface installations. Furthermore, understanding the migratory behavior of radionuclides in the reservoir may be useful for the characterization and sustainable management of the geothermal reservoir.

Tricca et al. (2001) describe the water-rock interactions as physico-chemical reactions between three phases: the aqueous phase, the solid minerals and a reactive surface layer with specific properties, area and thickness. The transfer rate of a radionuclide from the rock material into solution depends on: (a) the in-situ radioactive decay of its dissolved parent, (b) the desorption from the surface coating, (c) the alpha-recoil across the solid-liquid interface within a distance of several tens of nanometers, and (d) the dissolution of the aquifer solid. The removal of a radionuclide from the brine depends on: (a) its radioactive decay in solution, (b) the adsorption onto the surface layer, and (c) the incorporation into precipitates.

In the past, several mathematical solutions of simplified aquifer models dealing with naturally occurring radionuclides have been developed. Andrews et al. (1982, 1989) considered physico-chemical mechanisms for radionuclide supply and removal and calculated the timescale of water-rock interactions. However, the authors did not consider transport by advection. Krishnaswami et al. (1982) computed rate constants of sorption processes. Furthermore, they determined residence times of daughter nuclides by means of alpha-recoil input from ${ }^{222} \mathrm{Rn}$ activities, although without considering the effects of advective transport and mineral dissolution/precipitation. Davidson and Dickson (1986) suggested a model of uranium and radium isotopes transport including dispersive flow, but without considering precipitation and dissolution processes. Ku et al. (1992) proposed a model that accounts for radionuclide transport by advection and first-order kinetics, sorption-desorption, dissolution-precipitation of U-Th series radionuclides processes as well as the supply from alpha recoil. Tricca et al. $(2000,2001)$ suggested a model for the combined groundwater transport of naturally occurring $\mathrm{U}, \mathrm{Th}, \mathrm{Ra}$, and $\mathrm{Rn}$ isotopes with regards to advective transport as well as the physico-chemical processes of weathering, decay, alpha-recoil and sorption at the waterrock interface.

The present study is aimed at better understanding of the behavior of uranium and thorium series radionuclides in the Bruchsal geothermal brine. A comprehensive dataset was generated by frequent, long-term fluid sampling. Results of the geochemical surveys of major/minor elements as well as isotopic measurements of $\mathrm{U}$, Th, Ac, $\mathrm{Ra}, \mathrm{Rn}, \mathrm{Po}, \mathrm{Bi}$ and $\mathrm{Pb}$ are presented below. From the observed isotope disequilibria, water-rock interactions were investigated and their effects on radionuclide transport in the geothermal reservoir assessed. 
Since the modelling of radioactive disequilibria requires both types of information, the composition of the fluid and the solid phase, the authors refer to their previous work (Kölbel et al., 2020) where the Bruchsal reservoir rock was intensively examined based on cuttings from the geothermal boreholes. The modelling part of this study focuses on radium isotopes since their range in half-lives and their interrelation in the respective decay chain (cf. Fig. 4.1) allows the determination of waterrock interaction rates across different timescales.

\subsection{Geothermal context}

\subsubsection{Upper Rhine Graben (URG)}

The Upper Rhine Graben is part of the European Cenozoic Rift System that extends from the Mediterranean to the North Sea coast (Ziegler, 1992). It is characterized by an NNE-SSW striking extension structure with a length of approximately $300 \mathrm{~km}$ and a width of up to ca. $40 \mathrm{~km}$. The deep Hercynian basement consisting of Paleozoic granites is overlain by clastic sediments (sandstones) from Permian to Lower Triassic and by Middle Mesozoic to Cenozoic sediments. The base of the sediments in the center of the valley is ca. $3000 \mathrm{~m}$ deeper than at the valley shoulders (Ziegler, 1992).

The Upper Rhine Graben offers favorable conditions for the exploitation of geothermal energy. This is supported by spatially varying local heat flow anomalies and temperature anomalies at large depths. Most of the thermal anomalies are related to large-scale fluid circulation (Pribnow and Schellschmidt, 2000).

\subsubsection{Bruchsal site}

The Bruchsal geothermal site is located at the eastern main boundary fault of the Upper Rhine Graben. The geothermal power plant consists of a borehole doublet: an injection well (GB1) and a production well (GB2) located at a distance of $1.5 \mathrm{~km}$ from each other. Because of the complex tectonic structure, the reservoir section at the injection well differs from that of the production well with respect to depth and thickness. The geothermal reservoir is located in Permo-Triassic sedimentary rocks, characterized by large-scale normal faults of varying step heights, ranging between 20 and $350 \mathrm{~m}$ (Fig. 4.2). The hydrothermal reservoir is developed in a horizon comprising Middle Buntsandstein to Upper Rotliegend rocks (depth interval: 2220 - $2485 \mathrm{~m}$ ). The main inflow zones are located in the fractured zones of the Upper Permian at the depth interval between 2440 and $2470 \mathrm{~m}$ (Joachim et al., 1987). Hydrothermal alteration processes resulted in an almost complete transformation of the original feldspars into clay minerals, and in large quantities of Fe-hydroxides as limonite coatings around quartz grains (Kölbel et al., 2020). 


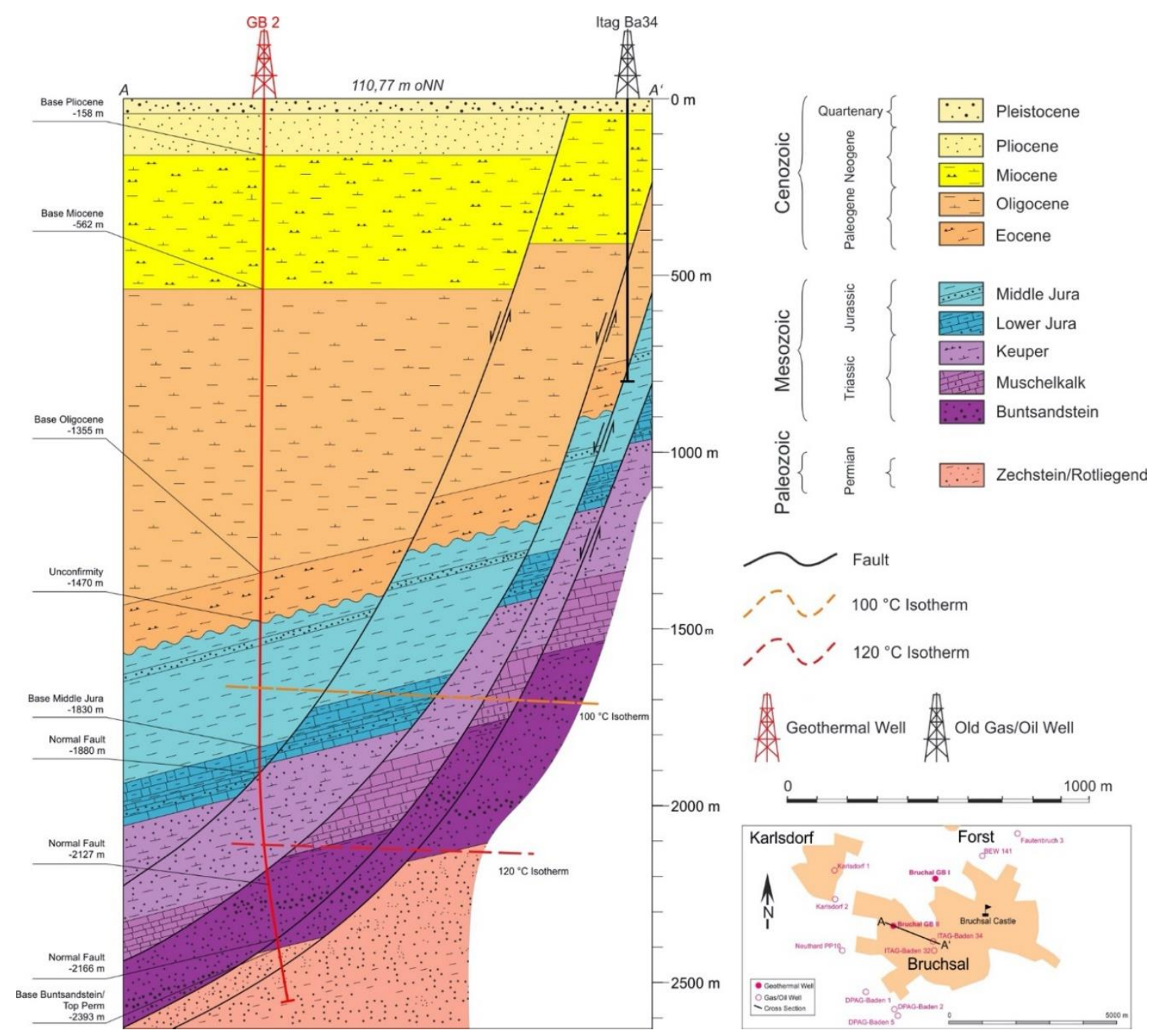

Fig. 4.2: Geological WNW - ESE cross section of the Bruchsal GB2 production well (Upper Rheine Valley, BadenWürttemberg, Germany). The figure represents a compilation of the work of Joachim et al. (1987) and Kölbel et al. (2020).

Borehole data of GB2 showed a bottom hole temperature of $134.7^{\circ} \mathrm{C}$ at $2542 \mathrm{~m}$ depth, corresponding to a geothermal gradient of $55 \mathrm{~K} \mathrm{~km}^{-1}$, and exceeding the typical average value for Central Europe of $30 \mathrm{~K} \mathrm{~km}^{-1}$. At present, the thermal water is produced with a flow rate of $24 \mathrm{~L} / \mathrm{s}$. Geochemical analysis of the $\mathrm{NaCl}$ brine showed high mineralization (TDS $\approx 130 \mathrm{~g} / \mathrm{l}$ ) including heavy metals and gases at a pH of 5 (Eggeling et al., 2013).

Activities of the primordial radionuclides ${ }^{238} \mathrm{U},{ }^{232} \mathrm{Th}$ and ${ }^{235} \mathrm{U}$ in the Upper Rotliegend amount to $18 \mathrm{~Bq} \mathrm{~kg}^{-1}, 17 \mathrm{~Bq} \mathrm{~kg}^{-1}$ and $0.8 \mathrm{~Bq} \mathrm{~kg}^{-1}$, respectively. These are relatively low, but rather typical values for sandstones (Gascoyne, 1992). 


\subsection{Methodology}

\subsubsection{Sampling and analytical methods}

Brine samples were collected at a sampling point close to the GB2 well head at the Bruchsal geothermal site. Hydrogeochemical investigations were conducted on thermal water abstracted from the production well GB2 during fluid circulation. The sampling period spanned between October 2016 and Mai 2017. In total 32 water samples were collected. Major ions and selected trace elements were analyzed in the samples by ICP-OES, ion chromatography and photometry. Temperature, $\mathrm{pH}$ and conductivity were measured on-site. ${ }^{238} \mathrm{U},{ }^{232} \mathrm{Th}$ and ${ }^{235} \mathrm{U}$ decay series radionuclides (Fig. 4.1) were analyzed in thirteen samples well GB2 to study their temporal variations in activities. The main research focus was on activity variations of radium isotopes $\left({ }^{224} \mathrm{Ra},{ }^{223} \mathrm{Ra},{ }^{228} \mathrm{Ra},{ }^{226} \mathrm{Ra}\right)$. Radiumsampling was conducted using gas-tight 1.2-liter Marinelli beakers (type G-130 G) prepared with $2.5 \mathrm{ml} 65 \% \mathrm{HNO}_{3}$ (suprapur) to inhibit precipitation of solids. Water samples were not filtered.

Gamma spectrometry surveys were carried out using a p-type HPGe coaxial detector of $30 \%$ efficiency (with respect to 3" x 3" $\mathrm{NaI}(\mathrm{Tl})$ detector). The germanium crystal had a diameter of 76 $\mathrm{mm}$. The detector was embedded in a $10 \mathrm{~cm}$ lead shield to protect against background radiation. Specific activities of radium isotopes were determined by gamma spectrometry allowing simultaneous measurements without further sample preparation. The list of the gamma rays used for the determination of activities of radium isotopes is reported in Table 4.1. Measurements $\left(M_{t 1}\right)$ were performed immediately after sample collection to determine activities of the short-lived radium daughters $\left({ }^{228} \mathrm{Ac},{ }^{212} \mathrm{~Pb},{ }^{214} \mathrm{~Pb}\right)$. The measurement duration $(\Delta t)$ was ca. 80,000 seconds $(=22.2$ hours). A second measurement $\left(M_{t 2}\right)$ was carried out after storing the sample for more than 42 hours, but less than 350 hours.

In addition, radionuclides of the ${ }^{238} \mathrm{U},{ }^{232} \mathrm{Th}$ and ${ }^{235} \mathrm{U}$ decay series were analyzed in an external certified laboratory. The activities of ${ }^{238} \mathrm{U},{ }^{234} \mathrm{U},{ }^{230} \mathrm{Th},{ }^{226} \mathrm{Ra},{ }^{210} \mathrm{Po},{ }^{235} \mathrm{U},{ }^{227} \mathrm{Ac},{ }^{223} \mathrm{Ra},{ }^{232} \mathrm{Th},{ }^{228} \mathrm{Th}$ and ${ }^{224} \mathrm{Ra}$ in the Bruchsal brine were measured by alpha spectrometry. ${ }^{228} \mathrm{Ra}$ was measured by beta counting. The accuracy of the gamma spectrometric method was checked by alpha spectrometry. Moreover, activities of uranium and thorium isotopes were determined which is not possible by gamma spectrometric measurements alone due to low U-Th-activities in the brine samples.

Reflecting the sample point at well head, the radionuclide activities measured in the lab were corrected by considering the travel time of produced fluids from reservoir to surface (lag time correction) as well as the elapsed time since fluid sampling.

Brine density was calculated according to Mao and Duan (2008), considering reservoir temperature and pressure as well as the molality of the $\mathrm{NaCl}$ brine $\left(\mathrm{T}=135{ }^{\circ} \mathrm{C}, \mathrm{p}=250 \mathrm{bar}, \mathrm{M}(\mathrm{NaCl})=2.1\right.$ $\mathrm{mol} / \mathrm{kg}$ ). The resulting brine density is $1023.24 \mathrm{~kg} / \mathrm{m}^{3}$. The activities of dissolved radionuclides are reported as disintegration rate per fluid-mass $\left(\right.$ atoms s$\left.~^{-1} \mathrm{~kg}^{-1}\right)$. 
Table 4.1: Overview of the nuclides used for determining Ra activities in the Bruchsal brine. Data about gamma ray energies (E $\gamma$ ) and intensities (I $\gamma$ ) originate from Condomines et al. (2010).

\begin{tabular}{|c|c|c|c|c|c|c|c|}
\hline $\begin{array}{l}\text { Nuclide of } \\
\text { interest }\end{array}$ & Half-life & $\begin{array}{l}\text { Measured } \\
\text { nuclide }\end{array}$ & $\mathrm{E} \gamma(\mathrm{keV})$ & $\mathrm{I} \gamma(\%)$ & $\begin{array}{l}\text { Interfering } \\
\text { nuclide }\end{array}$ & $\begin{array}{l}\mathrm{E} \gamma \\
(\mathrm{keV})\end{array}$ & $\mathrm{I} \gamma(\%)$ \\
\hline \multirow[t]{7}{*}{${ }^{226} \mathrm{Ra}$} & $1,600 \mathrm{y}$ & ${ }^{226} \mathrm{Ra}$ & 186.21 & 3.59 & & & \\
\hline & & ${ }^{214} \mathrm{~Pb}$ & 242.00 & 7.12 & & & \\
\hline & & & 295.22 & 18.15 & & & \\
\hline & & & 351.93 & 3.51 & ${ }^{211} \mathrm{Bi}$ & 351.06 & 12.91 \\
\hline & & ${ }^{214} \mathrm{Bi}$ & 609.31 & 44.60 & & & \\
\hline & & & $1,120.29$ & 14.70 & & & \\
\hline & & & $1,764.49$ & 15.10 & & & \\
\hline \multirow[t]{3}{*}{${ }^{228} \mathrm{Ra}$} & $5.75 \mathrm{y}$ & ${ }^{228} \mathrm{Ac}$ & 338.32 & 11.27 & ${ }^{223} \mathrm{Ra}$ & 338.28 & 2.79 \\
\hline & & & 911.07 & 25.80 & & & \\
\hline & & & 968.90 & 15.80 & & & \\
\hline \multirow[t]{2}{*}{${ }^{224} \mathrm{Ra}$} & $3.66 \mathrm{~d}$ & ${ }^{212} \mathrm{~Pb}$ & 238.63 & 43.30 & & & \\
\hline & & ${ }^{208} \mathrm{Tl}$ & 583.19 & 30.37 & & & \\
\hline \multirow[t]{3}{*}{${ }^{223} \mathrm{Ra}$} & $11.0 \mathrm{~d}$ & ${ }^{223} \mathrm{Ra}$ & 154.30 & 5.62 & ${ }^{228} \mathrm{Ac}$ & 153.98 & 0.72 \\
\hline & & & 269.60 & 13.73 & ${ }^{228} \mathrm{Ac}$ & 270.25 & 3.46 \\
\hline & & & & & ${ }^{219} \mathrm{Rn}$ & 271.23 & 10.8 \\
\hline
\end{tabular}

\subsubsection{Calculation of Ra activities from gamma spectrometry}

\subsubsection{1 $\quad{ }^{226} \mathrm{R} a$}

The ${ }^{226} \mathrm{Ra}$ activity was directly determined using its gamma ray energy at $186.2 \mathrm{keV}$. A possible interference with ${ }^{235} \mathrm{U}(185.7 \mathrm{keV})$ is negligible due to the low uranium activity shown by high resolution ICP-MS. Alternatively, the gamma-peaks of ${ }^{214} \mathrm{~Pb}$ and ${ }^{214} \mathrm{Bi}$ (daughters of ${ }^{226} \mathrm{Ra}$ and ${ }^{222} \mathrm{Rn}$, respectively) can be used to calculate the ${ }^{226} \mathrm{Ra}$ activity. In this case, the measurement can be performed after 20 days at the earliest, because this time is required to reach secular radioactive equilibrium (assuming no radon loss).

\subsubsection{2 $\quad{ }^{223} \mathrm{R} a$}

$154.3 \mathrm{keV}$ gamma ray energy was used to determine the ${ }^{223} \mathrm{Ra}$ activity. Here the presence of an interfering peak from ${ }^{228} \mathrm{Ac}$ has to be considered (cf. Table 4.1). According to Condomines et al. (2010), ${ }^{223} \mathrm{Ra}$ activity based on the $154.3 \mathrm{keV}$ peak can be corrected by: 


$$
\overline{\left({ }^{223} R a\right)}=\frac{C}{C_{S}} \cdot\left[\left({ }^{223} R a\right)_{S}+\left({ }^{228} A c\right)_{S} \cdot \frac{\varepsilon_{A c} I_{\gamma, A c}}{\varepsilon_{R a} I_{\gamma, R a}}\right]-\overline{\left({ }^{228} A c\right)} \cdot \frac{\varepsilon_{A c} I_{\gamma, A c}}{\varepsilon_{R a} I_{\gamma, R a}}
$$

where $\overline{\left({ }^{223} R a\right)}$ and $\overline{\left({ }^{228} \mathrm{Ac}\right)}$ are average activities integrated over the counting time. $C$ and $C_{S}$ are the counts for the sample and the standard, respectively. $\varepsilon_{A c}$ and $\varepsilon_{R a}$ are the apparent efficiencies for ${ }^{228} \mathrm{Ac}$ and ${ }^{223} \mathrm{Ra}$. $I_{\gamma, A c}$ and $I_{\gamma, R a}$ are gamma-ray intensities (cf. Table 4.1).

\subsubsection{3 $\quad{ }^{228} \mathrm{R} a$ and ${ }^{224} \mathrm{R} a$}

${ }^{228} \mathrm{Ra}$ and ${ }^{224} \mathrm{Ra}$ are part of the decay scheme of the ${ }^{232} \mathrm{Th}$ decay series (cf. Fig. 4.1). Measurements of both $\mathrm{Ra}$ isotopes include the count rates of their short-lived daughters ${ }^{228} \mathrm{Ac}\left(\mathrm{t}_{1 / 2}=6.13 \mathrm{~h}\right)$ and ${ }^{212} \mathrm{~Pb}\left(\mathrm{t}_{1 / 2}=10.64 \mathrm{~h}\right)$ which evolve through time during counting. The time-dependent evolution of the thorium decay series radionuclides is illustrated in Fig. 4.3 and can be described by a system of coupled differential equations.



Fig. 4.3: Several successive transformations within the ${ }^{232} \mathrm{Th}$ decay series for the Bruchsal brine: The dotted lines represent the simulated time-depended evolution over activities of ${ }^{228} \mathrm{Ra}$ and its daughters $\left({ }^{228} \mathrm{Ra} \rightarrow{ }^{228} \mathrm{Ac} \rightarrow{ }^{228} \mathrm{Th} \rightarrow\right.$ ${ }^{224} \mathrm{Ra}$ ). Time after sampling $(t)$ is found on the x-axis. Measured activities at the times of measuring $t_{1}$ and $t_{2}$ are plotted as dots. Calculations were performed by means of a CAS software (PTC Mathcad).

Their general solution was first given by Bateman (1910). A radioactive decay chain $\left(N_{1} \rightarrow N_{2} \rightarrow\right.$ $\ldots \rightarrow N_{i}$ ) with the decay constant $\lambda_{i}$ can be described by the following differential equations: 


$$
\begin{aligned}
& \frac{d N_{1}}{d t}=-\lambda_{1} N_{1} \\
& \frac{d N_{i}}{d t}=\lambda_{i-1} N_{i-1}-\lambda_{i} N_{i} \quad(i=2, n)
\end{aligned}
$$

Assuming zero concentrations of all daughters at time zero

$$
N_{1}(0) \neq 0 \quad \text { and } \quad N_{i}(0)=0 \quad \text { when } i>1
$$

Bateman (1910) expressed the concentration of $n$th radionuclide after time $t$ as:

$$
N_{n}(t)=\frac{N_{1}(0)}{\lambda_{n}} \sum_{i=1}^{n} \lambda_{i} \alpha_{i} \exp \left[-\lambda_{i} t\right]
$$

where the coefficients are calculated by

$$
\alpha_{i}=\prod_{j=1}^{n} \frac{\lambda_{j}}{\left(\lambda_{j}-\lambda_{i}\right)} \quad(j \neq i)
$$

Degering and Köhler (2011) adjusted Eqn. (4.4) to the time averaged activity expressed as

$$
\overline{A_{l}\left(t_{d}, \Delta t\right)}=\frac{1}{\Delta t} \int_{t_{d}}^{t_{d}+\Delta t} A_{i}(t) d t=\sum_{j=1}^{i} \alpha_{i j} \overline{\tau_{J}\left(t_{d}, \Delta t\right)}
$$

where

$$
\begin{aligned}
& \overline{\tau_{j}\left(t_{d}, \Delta t\right)}=\frac{1}{\Delta t \lambda_{j}} \tau_{j}\left(t_{d}\right)\left(1-\tau_{j}(\Delta t)\right. \\
& \tau_{j}(t)=e^{-\lambda_{j} t}
\end{aligned}
$$

The mathematical solution for the Bruchsal site was computed by employing Mathcad ${ }^{\circledR}$. For better understanding, index notation of ${ }^{228} \mathrm{Ra}=\mathrm{A}_{1} ;{ }^{228} \mathrm{Ac}=\mathrm{A}_{2},{ }^{228} \mathrm{Th}=\mathrm{A}_{3}$ and ${ }^{224} \mathrm{Ra}=\mathrm{A}_{4}$ are used from here on.

The activity of ${ }^{228} \mathrm{Ra}$ at sampling time $(\mathrm{t}=0)$ was determined from the ${ }^{228} \mathrm{Ac}$ activity after a waiting period $t_{d}$ of minimum 42 hours after sampling:

$$
A_{1}(0)=\frac{A_{2}\left(t_{d}, \Delta t\right)}{\tau_{1}\left(t_{d}, \Delta t\right)}
$$


${ }^{224} \mathrm{Ra}$ was determined gamma-rays emitted by the daughter nuclides ${ }^{212} \mathrm{~Pb}(238.6 \mathrm{keV})$ and ${ }^{208} \mathrm{Tl}$ $(583.1 \mathrm{keV})$ with the ${ }^{212} \mathrm{~Pb} /{ }^{224} \mathrm{Ra}$ ratio reaching a steady state value of 1.14 after ca. 100 hours:

$$
\begin{aligned}
& A_{4}(0)=\frac{1}{\overline{\tau_{4}\left(t_{d}, \Delta t\right)}}\left(\overline{A_{4}\left(t_{d}, \Delta t\right)}-\left(c_{1} A_{1}(0)+c_{3} A_{3}(0)\right)\right. \\
& c_{1}=\alpha_{41} \alpha_{31}\left(\overline{\tau_{1}\left(t_{d}, \Delta t\right)}-\overline{\tau_{4}\left(t_{d}, \Delta t\right)}\right)-\alpha_{43} \alpha_{31}\left(\overline{\tau_{3}\left(t_{d}, \Delta t\right)}-\overline{\tau_{4}\left(t_{d}, \Delta t\right)}\right) \\
& c_{3}=\alpha_{43}\left(\overline{\tau_{3}\left(t_{d}, \Delta t\right)}-\overline{\tau_{4}\left(t_{d}, \Delta t\right)}\right) \\
& c_{4}=\overline{\tau_{4}\left(t_{d}, \Delta t\right)}
\end{aligned}
$$

\subsection{Results}

\subsubsection{Major and trace elements}

Field parameter and major and minor element data are reported in

Table 4.2. The Bruchsal brine is highly concentrated in chloride, sodium and other alkali metals and alkaline earth metals, containing up to $131 \mathrm{~g} / 1$ of total dissolved solids (TDS). Furthermore, the brine is enriched in sulfate and hydrogen carbonate as well as heavy metals such as lead, arsenic, and cadmium. In contrast, the concentration of organic compounds is low. Eh-pH conditions are difficult to determine because of the change in pressure and temperature between reservoir and the sampling location at ground level. At the sampling point, $\mathrm{pH}$ values range between 5.0 and 5.9 [], while Eh values are relatively constant at ca. $81 \mathrm{mV}$ (on average).

Table 4.2: Physical parameters and chemical composition of the Bruchsal geothermal fluid. Mean values are based on the analysis of 32 samples.

\begin{tabular}{lcccccc}
\hline & $\begin{array}{c}\text { Flow rate } \\
{[1 / \mathrm{s}]}\end{array}$ & $\begin{array}{c}\mathrm{T}_{\text {sampling }} \\
{\left[{ }^{\circ} \mathrm{C}\right]}\end{array}$ & $\begin{array}{c}\text { Conductivity } \\
\text { at } 25{ }^{\circ} \mathrm{C} \\
{[\mathrm{mS} / \mathrm{cm}]}\end{array}$ & $\begin{array}{c}\mathrm{pH} \\
{[}\end{array}$ & $\begin{array}{c}\mathrm{Eh} \\
{[\mathrm{mV}]}\end{array}$ & $\begin{array}{c}\text { TDS } \\
{[\mathrm{g} / \mathrm{l}]}\end{array}$ \\
\hline mean & 25.5 & 21.5 & 155.6 & 5.3 & 81 & 131 \\
$\min$ & 23.3 & 18.7 & 152.5 & 5.0 & 91 & 127 \\
$\max$ & 29.5 & 26.0 & 158.1 & 5.9 & 72 & 135
\end{tabular}




\begin{tabular}{|c|c|c|c|c|c|c|}
\hline & $\begin{array}{c}\mathrm{K} \\
{[\mathrm{mg} / \mathrm{l}]}\end{array}$ & $\begin{array}{c}\mathrm{Na} \\
{[\mathrm{mg} / \mathrm{l}]}\end{array}$ & $\begin{array}{c}\mathrm{Ca} \\
{[\mathrm{mg} / \mathrm{l}]}\end{array}$ & $\begin{array}{c}\mathrm{Mg} \\
{[\mathrm{mg} / \mathrm{l}]}\end{array}$ & $\begin{array}{c}\mathrm{Cl} \\
{[\mathrm{mg} / \mathrm{l}]}\end{array}$ & $\begin{array}{c}\mathrm{SO}_{4} \\
{[\mathrm{mg} / \mathrm{l}]}\end{array}$ \\
\hline mean & 3,523 & 40,543 & 9,478 & 397 & 74,910 & 339 \\
\hline $\min$ & 3,309 & 38,134 & 9,052 & 361 & 73,250 & 299 \\
\hline \multirow[t]{2}{*}{$\max$} & 3,642 & 42,765 & 9,956 & 437 & 76,166 & 492 \\
\hline & $\begin{array}{c}\mathrm{Sr} \\
{[\mathrm{mg} / \mathrm{l}]}\end{array}$ & $\begin{array}{c}\mathrm{Ba} \\
{[\mathrm{mg} / \mathrm{l}]}\end{array}$ & $\begin{array}{c}\mathrm{Br} \\
{[\mathrm{mg} / \mathrm{l}]}\end{array}$ & $\begin{array}{l}\mathrm{HCO}_{3} \\
{[\mathrm{mg} / \mathrm{l}]}\end{array}$ & $\begin{array}{c}\mathrm{Fe} \\
{[\mathrm{mg} / \mathrm{l}]}\end{array}$ & $\begin{array}{c}\mathrm{Fe}^{2+} \\
{[\mathrm{mg} / \mathrm{l}]}\end{array}$ \\
\hline mean & 387 & 9.2 & 312 & 341 & 50.1 & 32.7 \\
\hline Min & 362 & 6.7 & 284 & 255 & 49.2 & 26.3 \\
\hline \multirow[t]{2}{*}{$\max$} & 400 & 9.6 & 341 & 374 & 51.5 & 44.4 \\
\hline & $\begin{array}{c}\mathrm{Fe}^{3+} \\
{[\mathrm{mg} / \mathrm{l}]}\end{array}$ & $\begin{array}{c}\mathrm{Mn} \\
{[\mathrm{mg} / \mathrm{l}]}\end{array}$ & $\begin{array}{c}\mathrm{Pb} \\
{[\mathrm{mg} / \mathrm{l}]}\end{array}$ & $\begin{array}{c}\text { As } \\
{[\mathrm{mg} / \mathrm{l}]}\end{array}$ & $\begin{array}{c}\mathrm{Al} \\
{[\mathrm{mg} / \mathrm{l}]}\end{array}$ & $\begin{array}{c}\mathrm{Cd} \\
{[\mathrm{mg} / \mathrm{l}]}\end{array}$ \\
\hline mean & 19.4 & 25.7 & 3.0 & 9.7 & 1.8 & 0.2 \\
\hline $\min$ & 12.2 & 23.6 & 1.5 & 6.0 & 1.1 & 0.1 \\
\hline $\max$ & 24.0 & 27.5 & 5.5 & 12.0 & 2.6 & 0.4 \\
\hline
\end{tabular}

\subsubsection{U-Th decay series radionuclides}

Results of the investigated radionuclides are listed in Table 4.3. Relative uncertainties are quoted in percentage as two-standard deviations based on counting statistics. Generally, $\mathrm{Ra}\left({ }^{226} \mathrm{Ra},{ }^{228} \mathrm{Ra},{ }^{224} \mathrm{Ra}\right.$, $\left.{ }^{223} \mathrm{Ra}\right), \mathrm{Rn}\left({ }^{222} \mathrm{Rn}\right)$ and $\mathrm{Pb}\left({ }^{210} \mathrm{~Pb},{ }^{212} \mathrm{~Pb}\right)$ are rather mobile in geothermal brine, while $\mathrm{U}\left({ }^{238} \mathrm{U},{ }^{234} \mathrm{U}\right.$, $\left.{ }^{235} \mathrm{U}\right)$, Th $\left({ }^{232} \mathrm{Th},{ }^{228} \mathrm{Th},{ }^{230} \mathrm{Th}\right), \mathrm{Po}\left({ }^{210} \mathrm{Po}\right)$ and $\mathrm{Ac}\left({ }^{227} \mathrm{Ac},{ }^{228} \mathrm{Ac}\right)$ are less mobile e.g. due to their adsorptive behavior or co-precipitation (Hammond et al, 1988). Consequently, significant radioactive disequilibria form within the natural decay series (Fig. 4.4)

Activities of Th $\left({ }^{230} \mathrm{Th},{ }^{232} \mathrm{Th}\right.$ and $\left.{ }^{228} \mathrm{Th}\right)$ and $\mathrm{U}\left({ }^{238} \mathrm{U},{ }^{234} \mathrm{U},{ }^{235} \mathrm{U}\right)$ isotopes in brine were below the limit of analytical determination. This suggests that in the reservoir uranium preferentially exists in the tetravalent state, forming insoluble phases such as $\mathrm{UO}_{2}$ and $\mathrm{USiO}_{4}$. Thorium is only stable in the tetravalent state, irrespective of the redox conditions, uranium requires a reducing environment to become tetravalent as in deep geothermal reservoirs (Attendorn and Bowen, 1997). 
Table 4.3: ${ }^{238} \mathrm{U},{ }^{232} \mathrm{Th}$ and ${ }^{235} \mathrm{U}$ decay series radionuclides in the Bruchsal brine samples. Relative uncertainties are quoted in percentage $( \pm 2 \sigma$ from counting statistics).

\begin{tabular}{|c|c|c|c|c|c|}
\hline Decay series & Radionuclide & $\begin{array}{l}\text { Specific activity } \\
\left(\mathrm{Bq} \mathrm{kg}^{-1}\right)\end{array}$ & $\begin{array}{l}\text { Relative uncer- } \\
\text { tainty }(\%)\end{array}$ & $\begin{array}{l}\text { Number of } \\
\text { samples }\end{array}$ & Method \\
\hline \multirow{7}{*}{$\begin{array}{l}\text { Uranium } \\
\text { series }\end{array}$} & ${ }^{238} \mathrm{U}$ & 0.012 & 59 & 1 & $\alpha$-spectrometry \\
\hline & ${ }^{234} \mathrm{U}$ & 0.01 & 66 & 1 & $\alpha$-spectrometry \\
\hline & ${ }^{230} \mathrm{Th}$ & $<0.08$ & & 1 & $\alpha$-spectrometry \\
\hline & ${ }^{226} \mathrm{Ra}$ & 29.0 & 8.3 & 13 & $\gamma$ - spectrometry \\
\hline & ${ }^{222} \mathrm{Rn}$ & 37.8 & 7.4 & 1 & $\gamma$-spectrometry \\
\hline & ${ }^{210} \mathrm{~Pb}$ & 25.8 & 26 & 12 & $\gamma$ - spectrometry \\
\hline & ${ }^{210} \mathrm{Po}$ & $<0.1$ & & 1 & $\alpha$-spectrometry \\
\hline \multirow{3}{*}{$\begin{array}{l}\text { Actinium } \\
\text { series }\end{array}$} & $235 \mathrm{U}$ & $<0.004$ & & 1 & $\alpha$-spectrometry \\
\hline & ${ }^{227} \mathrm{Ac}$ & $<0.1$ & & 1 & $\alpha$-spectrometry \\
\hline & ${ }^{223} \mathrm{Ra}$ & 0.47 & 60 & 13 & $\gamma$-spectrometry \\
\hline \multirow{6}{*}{$\begin{array}{l}\text { Thorium } \\
\text { series }\end{array}$} & ${ }^{232} \mathrm{Th}$ & $<0.01$ & & 1 & $\alpha$-spectrometry \\
\hline & ${ }^{228} \mathrm{Ra}$ & 15.9 & 5.8 & 13 & $\gamma$ - spectrometry \\
\hline & ${ }^{228} \mathrm{Ac}$ & 6.1 & 27 & 2 & $\gamma$ - spectrometry \\
\hline & ${ }^{228} \mathrm{Th}$ & $<0.02$ & & 1 & $\alpha$-spectrometry \\
\hline & ${ }^{224} \mathrm{Ra}$ & 10.3 & 7.5 & 13 & $\gamma$ - spectrometry \\
\hline & ${ }^{212} \mathrm{~Pb}$ & 15.7 & 6.4 & 2 & $\gamma$ - spectrometry \\
\hline
\end{tabular}

In contrast, high levels of activity were observed for Ra isotopes $\left({ }^{226} \mathrm{Ra},{ }^{228} \mathrm{Ra},{ }^{224} \mathrm{Ra},{ }^{223} \mathrm{Ra}\right),{ }^{222} \mathrm{Rn}$ and $\mathrm{Pb}$ isotopes $\left({ }^{210} \mathrm{~Pb},{ }^{212} \mathrm{~Pb}\right)$. Their activities are several orders of magnitude higher than those of their thorium progenitors. ${ }^{222} \mathrm{Rn}$, is in slight excess relative to its parent ${ }^{226} \mathrm{Ra}$.

Isotopes of $\mathrm{Pb}\left({ }^{210} \mathrm{~Pb}\right.$ and $\left.{ }^{212} \mathrm{~Pb}\right)$ have high solubilities resulting in fluid activities $>15 \mathrm{~Bq} \mathrm{~kg}^{-1} \cdot{ }^{210} \mathrm{~Pb}$ $\left(A_{210 \mathrm{~Pb}}=25.8 \mathrm{~Bq} \mathrm{~kg}{ }^{-1}\right)$ was found to be deficient relative to its progenitors ${ }^{222} \mathrm{Rn}\left(A_{222 \mathrm{R} n}=37.8 \mathrm{~Bq}\right.$ $\left.\mathrm{kg}^{-1}\right)$ and ${ }^{226} \mathrm{Ra}\left(A_{226 \mathrm{Ra}}=29.0 \mathrm{~Bq} \mathrm{~kg}^{-1}\right)$, but still in the same order of magnitude indicating that $\mathrm{Ra}$, $\mathrm{Rn}$ and $\mathrm{Pb}$ have a comparable mobility in the Bruchsal geothermal system.

The activity of ${ }^{210} \mathrm{Po}$ is below the limit of determination. The low ${ }^{210} \mathrm{Po}$ activity in comparison to its parent ${ }^{210} \mathrm{~Pb}$ suggests removal of ${ }^{210} \mathrm{Po}$ from the brine. The same applies to isotopes of actinium: The short-lived ${ }^{228} \mathrm{Ac}\left(A_{228 \mathrm{Ac}}=6.1 \mathrm{~Bq} \mathrm{~kg}^{-1}\right)$ of the thorium decay series is deficient relative to its 

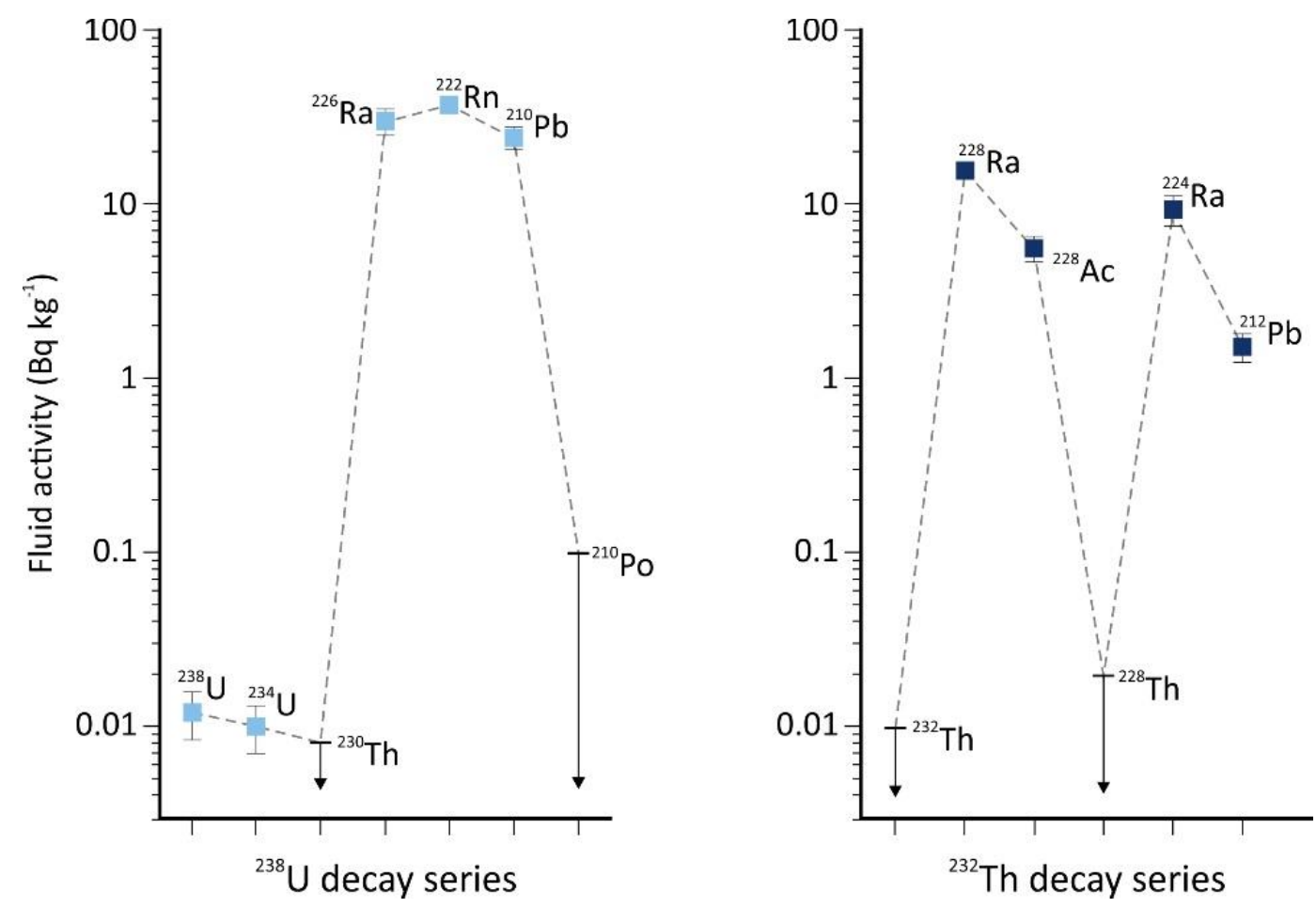

Fig. 4.4: Uranium and thorium series disequilibria: specific activity of ${ }^{238} \mathrm{U}$ and ${ }^{232} \mathrm{Th}$ and their decay products measured in the Bruchsal brine. Error bars represent uncertainties due to counting statistics $( \pm 2 \sigma) .{ }^{230} \mathrm{Th},{ }^{210} \mathrm{Po},{ }^{233} \mathrm{Th}$ and ${ }^{228} \mathrm{Th}$ activities are upper limits. Radionuclide movement down the decay chain is from left to right.

parent ${ }^{228} \mathrm{Ra}\left(A_{228 R a}=15.9 \mathrm{~Bq} \mathrm{~kg}^{-1}\right) .{ }^{227} \mathrm{Ac}$, an isotope of the ${ }^{235} \mathrm{U}$ decay series, was found to have a lower activity than its daughter ${ }^{223} \mathrm{R}$ a indicating the rapid depletion of ${ }^{227} \mathrm{Ac}$ from brine.

The rock/brine activity ratio $\left(R_{c}\right)$ is a measure of the relative mobility of the isotopes (Zukin et al., 1987). Values for $R_{c}$ were determined based on analyses of brine (Table 4.3) and rock samples from borehole cuttings (Kölbel et al. 2020). The results are summarized in Table 4.4. High $R_{c}$ values in the order of magnitude of $10^{2}$ for isotopes of $\mathrm{U}\left({ }^{238} \mathrm{U},{ }^{234} \mathrm{U},{ }^{235} \mathrm{U}\right)$ and $\mathrm{Th}\left({ }^{232} \mathrm{Th},{ }^{228} \mathrm{Th},{ }^{230} \mathrm{Th}\right)$ reflect the affinity of the nuclides to the surface of the solids for both elements. In turn, isotopes of $\mathrm{Ra}$ $\left.{ }^{226} \mathrm{Ra},{ }^{228} \mathrm{Ra},{ }^{224} \mathrm{Ra},{ }^{223} \mathrm{Ra}\right)$ and $\mathrm{Pb}\left({ }^{210} \mathrm{~Pb}\right.$ and $\left.{ }^{212} \mathrm{~Pb}\right)$ have lower $\mathrm{R}_{c}$ values and therefore, a relatively high mobility in the investigated geothermal system is implied.

${ }^{228} \mathrm{Th}$ (a decay product of ${ }^{232} \mathrm{Th}$ ) has a rather low $\mathrm{R}_{c}$ value compared to ${ }^{232} \mathrm{Th}$. This reflects a better accessibility of ${ }^{228} \mathrm{Th}$ to the geothermal brine because of the good solubility of its ${ }^{228} \mathrm{Ra}$ progenitor. Following the decay of ${ }^{228} \mathrm{Ra}$, ${ }^{228} \mathrm{Th}$ is adsorbed onto grain surfaces producing a comparatively high activity compared to the activity of the ${ }^{232} \mathrm{Th}$ isotope.

In summary, isotopes of thorium, polonium and actinium generally display low levels of activity in the brine as a result of their poor solubility. The processes of removal of the above isotopes from solution are most likely adsorption and/or co-precipitation. 
Table 4.4: $R_{c}$ values of Th- $U$ decay series radionuclides. $R_{c}$ is defined as the ratio of rock activity relative to average fluid activity (cf. Table 4.3).

\begin{tabular}{llll}
\hline Isotope & $\begin{array}{l}\text { Rock activity } \\
\left(\mathrm{Bq} \mathrm{kg}^{-1}\right)\end{array}$ & $\mathrm{R}_{c}$ values & Half-life \\
\hline${ }^{238 \mathrm{U}}$ & 18.0 & $1.5 \times 10^{3}$ & $447 \times 10^{9} \mathrm{v}$ \\
${ }^{235} \mathrm{U}$ & 0.8 & $>2.1 \times 10^{2}$ & $7.04 \times 10^{8} \mathrm{v}$ \\
${ }^{234} \mathrm{U}$ & 19.0 & $1.9 \times 10^{3}$ & $2.46 \times 10^{5} \mathrm{v}$ \\
\hline${ }^{232} \mathrm{Th}$ & 17.0 & $>1.7 \times 10^{3}$ & $141 \times 10^{10} \mathrm{v}$ \\
${ }^{230} \mathrm{Th}$ & 19.0 & $>2.4 \times 10^{2}$ & $7.54 \times 10^{4} \mathrm{y}$ \\
${ }^{228} \mathrm{Th}$ & 19.0 & $>9.5 \times 10^{2}$ & $1.91 \mathrm{y}$ \\
\hline${ }^{226} \mathrm{Ra}$ & 43.0 & 1.5 & $1600 \mathrm{y}$ \\
${ }^{228} \mathrm{Ra}$ & 17.0 & 1.1 & $5.75 \mathrm{y}$ \\
${ }^{223} \mathrm{Ra}$ & 0.8 & 1.8 & $11.43 \mathrm{~d}$ \\
${ }^{224} \mathrm{Ra}$ & 19.0 & 1.7 & $3.66 \mathrm{~d}$ \\
\hline${ }^{210} \mathrm{~Pb}$ & 37.0 & 1.4 & $22.30 \mathrm{y}$ \\
${ }^{212} \mathrm{~Pb}$ & 19.0 & 1.2 & $10.64 \mathrm{~h}$ \\
\hline
\end{tabular}

\subsubsection{Radium isotopes}

Radium isotopes $\left({ }^{226} \mathrm{Ra},{ }^{228} \mathrm{Ra},{ }^{224} \mathrm{Ra},{ }^{223} \mathrm{Ra}\right)$ were measured several times during power plant operation. Radium activities as well as activity ratios of $\mathrm{Ra}$ isotopes $\left({ }^{228} \mathrm{Ra} /{ }^{226} \mathrm{Ra},{ }^{224} \mathrm{Ra} /{ }^{228} \mathrm{Ra},{ }^{223} \mathrm{Ra} /{ }^{226} \mathrm{Ra}\right)$ are listed in Table 4.5.

The highest activities range from 27 to $32 \mathrm{~Bq} \mathrm{~kg}^{-1}$ and are identified for the long-lived ${ }^{226} \mathrm{Ra}$ isotope. ${ }^{228} \mathrm{Ra},{ }^{224} \mathrm{Ra}$ and ${ }^{223} \mathrm{Ra}$ activities show lower values that vary between 15 and $17 \mathrm{~Bq} \mathrm{~kg}^{-1}, 9$ and $12 \mathrm{~Bq}$ $\mathrm{kg}^{-1}$, and 0.3 and $0.7 \mathrm{~Bq} \mathrm{~kg}^{-1}$, respectively. Variation in activity levels of the radium isotopes are likely a consequence of analytical uncertainties and steady state in activities of the radium isotopes can be assumed.

This observation is also made with the activity ratios of radium that are principally constant within $2 \sigma$ analytical uncertainties during the period of sampling: ${ }^{228} \mathrm{Ra} /{ }^{226} \mathrm{Ra},{ }^{224} \mathrm{Ra} /{ }^{228} \mathrm{Ra}$ and ${ }^{223} \mathrm{Ra} /{ }^{226} \mathrm{Ra}$ ratios display mean values of $0.55 \pm 0.07,0.65 \pm 0.07$ and $0.02 \pm 0.01$.

Gamma spectrometry results from earlier analysis (cf. Table 4.6), however, indicate that Ra activity in brine has increased over the past years. This particularly applies to ${ }^{226} \mathrm{Ra}$ and ${ }^{228} \mathrm{Ra}$ whose activities both increased by $25 \%$ resulting in a constant ${ }^{228} \mathrm{Ra} /{ }^{226} \mathrm{Ra}$ ratio $(0.53 \pm 0.09$ in 1986 and $0.55 \pm$ 0.07 in 2016/17). However, the short-lived ${ }^{224} \mathrm{Ra}$ activity has been more or less stable since 1986 resulting in a decrease in the ${ }^{224} \mathrm{Ra} /{ }^{228} \mathrm{Ra}$ activity ratio over the past 30 years from 0.75 in 1986 to 0.65 in 2016. 
Table 4.5: Radium activities and activity ratios in brine collected from GB2 well (measurement campaign 2016/2017). Measured values are reported in $\mathrm{Bq} \mathrm{kg}^{-1}$. The quoted errors are $2 \sigma$-deviations derived from counting statistics.

\begin{tabular}{|c|c|c|c|c|c|c|c|c|}
\hline $\begin{array}{l}\text { Sample } \\
\text { No. }\end{array}$ & $\begin{array}{l}\text { Sampling } \\
\text { date }\end{array}$ & ${ }^{226} \mathrm{Ra}$ & ${ }^{228} \mathrm{Ra}$ & ${ }^{224} \mathrm{Ra}$ & ${ }^{223} \mathrm{Ra}$ & ${ }^{228} \mathrm{Ra} /{ }^{226} \mathrm{Ra}$ & ${ }^{224} \mathrm{Ra} /{ }^{228} \mathrm{Ra}$ & ${ }^{223} \mathrm{Ra} /{ }^{226} \mathrm{Ra}$ \\
\hline $\begin{array}{l}\text { GB2-p1z1- } \\
001\end{array}$ & 13.10 .2016 & $\begin{array}{l}28.8 \\
\pm 2.4\end{array}$ & $\begin{array}{l}15.2 \\
\pm 0.8\end{array}$ & $\begin{array}{l}10.6 \\
\pm 0.6\end{array}$ & $\begin{array}{l}0.41 \\
\pm 0.25\end{array}$ & $\begin{array}{l}0.53 \\
\pm 0.07\end{array}$ & $\begin{array}{l}0.70 \\
\pm 0.08\end{array}$ & $\begin{array}{l}0.01 \\
\pm 0.01\end{array}$ \\
\hline $\begin{array}{l}\text { GB2-p1z1- } \\
002\end{array}$ & 18.10 .2016 & $\begin{array}{l}27.6 \\
\pm 2.6\end{array}$ & $\begin{array}{l}14.9 \\
\pm 0.8\end{array}$ & $\begin{array}{l}10.0 \\
\pm 0.4\end{array}$ & $\begin{array}{l}0.37 \\
\pm 0.22\end{array}$ & $\begin{array}{l}0.54 \\
\pm 0.08\end{array}$ & $\begin{array}{l}0.67 \\
\pm 0.06\end{array}$ & $\begin{array}{l}0.01 \\
\pm 0.01\end{array}$ \\
\hline $\begin{array}{l}\text { GB2-p1z1- } \\
003\end{array}$ & 24.10 .2016 & $\begin{array}{l}28.1 \\
\pm 2.4\end{array}$ & $\begin{array}{l}15.5 \\
\pm 0.8\end{array}$ & $\begin{array}{l}10.4 \\
\pm 0.4\end{array}$ & $\begin{array}{l}0.48 \\
\pm 0.29\end{array}$ & $\begin{array}{l}0.55 \\
\pm 0.08\end{array}$ & $\begin{array}{l}0.67 \\
\pm 0.06\end{array}$ & $\begin{array}{l}0.02 \\
\pm 0.01\end{array}$ \\
\hline $\begin{array}{l}\text { GB2-p1z1- } \\
004\end{array}$ & 28.10 .2016 & $\begin{array}{l}29.3 \\
\pm 2.8\end{array}$ & $\begin{array}{l}16.4 \\
\pm 0.9\end{array}$ & $\begin{array}{l}11.5 \\
\pm 0.6\end{array}$ & $\begin{array}{l}0.53 \\
\pm 0.32\end{array}$ & $\begin{array}{l}0.56 \\
\pm 0.08\end{array}$ & $\begin{array}{l}0.70 \\
\pm 0.08\end{array}$ & $\begin{array}{l}0.02 \\
\pm 0.01\end{array}$ \\
\hline $\begin{array}{l}\text { GB2-p1z1- } \\
005\end{array}$ & 04.11 .2016 & $\begin{array}{l}27.2 \\
\pm 2.2\end{array}$ & $\begin{array}{l}16.1 \\
\pm 0.8\end{array}$ & $\begin{array}{l}9.0 \\
\pm 2.0\end{array}$ & $\begin{array}{l}0.74 \\
\pm 0.44\end{array}$ & $\begin{array}{l}0.59 \\
\pm 0.08\end{array}$ & $\begin{array}{l}0.56 \\
\pm 0.15\end{array}$ & $\begin{array}{l}0.03 \\
\pm 0.02\end{array}$ \\
\hline $\begin{array}{l}\text { GB2-p1z1- } \\
006\end{array}$ & 16.11.2016 & $\begin{array}{l}28.0 \\
\pm 2.4\end{array}$ & $\begin{array}{l}15.3 \\
\pm 0.8\end{array}$ & $\begin{array}{l}10.4 \\
\pm 0.6\end{array}$ & $\begin{array}{l}0.51 \\
\pm 0.31\end{array}$ & $\begin{array}{l}0.55 \\
\pm 0.08\end{array}$ & $\begin{array}{l}0.68 \\
\pm 0.07\end{array}$ & $\begin{array}{l}0.02 \\
\pm 0.01\end{array}$ \\
\hline $\begin{array}{l}\text { GB2-p1z1- } \\
007\end{array}$ & 17.01 .2017 & $\begin{array}{l}29.5 \\
\pm 2.4\end{array}$ & $\begin{array}{l}15.5 \\
\pm 0.8\end{array}$ & $\begin{array}{l}10.1 \\
\pm 0.8\end{array}$ & $\begin{array}{l}0.61 \\
\pm 0.37\end{array}$ & $\begin{array}{l}0.53 \\
\pm 0.07\end{array}$ & $\begin{array}{l}0.65 \\
\pm 0.09\end{array}$ & $\begin{array}{l}0.02 \\
\pm 0.01\end{array}$ \\
\hline $\begin{array}{l}\text { GB2-p1z1- } \\
008\end{array}$ & 04.04 .2017 & $\begin{array}{l}30.3 \\
\pm 2.2\end{array}$ & $\begin{array}{l}16.0 \\
\pm 0.7\end{array}$ & $\begin{array}{l}10.3 \\
\pm 0.4\end{array}$ & $\begin{array}{l}0.32 \\
\pm 0.19\end{array}$ & $\begin{array}{l}0.53 \\
\pm 0.06\end{array}$ & $\begin{array}{l}0.64 \\
\pm 0.05\end{array}$ & $\begin{array}{l}0.01 \\
\pm 0.01\end{array}$ \\
\hline $\begin{array}{l}\text { GB2-p1z1- } \\
009\end{array}$ & 11.04 .2017 & $\begin{array}{l}32.0 \\
\pm 2.4\end{array}$ & $\begin{array}{l}16.7 \\
\pm 0.9\end{array}$ & $\begin{array}{l}9.7 \\
\pm 1.0\end{array}$ & $\begin{array}{l}0.36 \\
\pm 0.22\end{array}$ & $\begin{array}{l}0.52 \\
\pm 0.07\end{array}$ & $\begin{array}{l}0.58 \\
\pm 0.09\end{array}$ & $\begin{array}{l}0.01 \\
\pm 0.01\end{array}$ \\
\hline $\begin{array}{l}\text { GB2-p1z1- } \\
010\end{array}$ & 27.04 .2017 & $\begin{array}{l}29.9 \\
\pm 2.4\end{array}$ & $\begin{array}{l}16.0 \\
\pm 0.8\end{array}$ & $\begin{array}{l}10.6 \\
\pm 0.6\end{array}$ & $\begin{array}{l}0.44 \\
\pm 0.26\end{array}$ & $\begin{array}{l}0.54 \\
\pm 0.07\end{array}$ & $\begin{array}{l}0.66 \\
\pm 0.07\end{array}$ & $\begin{array}{l}0.02 \\
\pm 0.01\end{array}$ \\
\hline $\begin{array}{l}\text { GB2-p1z1- } \\
011\end{array}$ & 02.05 .2017 & $\begin{array}{l}27.8 \\
\pm 2.4\end{array}$ & $\begin{array}{l}16.4 \\
\pm 0.8\end{array}$ & $\begin{array}{l}10.7 \\
\pm 0.4\end{array}$ & $\begin{array}{l}0.50 \\
\pm 0.30\end{array}$ & $\begin{array}{l}0.59 \\
\pm 0.08\end{array}$ & $\begin{array}{l}0.65 \\
\pm 0.06\end{array}$ & $\begin{array}{l}0.02 \\
\pm 0.01\end{array}$ \\
\hline $\begin{array}{l}\text { GB2-p1z1- } \\
012\end{array}$ & 08.05 .2017 & $\begin{array}{l}28.9 \\
\pm 2.4\end{array}$ & $\begin{array}{l}16.2 \\
\pm 0.8\end{array}$ & $\begin{array}{l}11.1 \\
\pm 0.4\end{array}$ & $\begin{array}{l}0.43 \\
\pm 0.26\end{array}$ & $\begin{array}{l}0.56 \\
\pm 0.07\end{array}$ & $\begin{array}{l}0.69 \\
\pm 0.06\end{array}$ & $\begin{array}{l}0.02 \\
\pm 0.01\end{array}$ \\
\hline $\begin{array}{l}\text { GB2-p1z1- } \\
013\end{array}$ & 15.05 .2017 & $\begin{array}{l}30.0 \\
\pm 2.4\end{array}$ & $\begin{array}{l}16.1 \\
\pm 0.8\end{array}$ & $\begin{array}{l}10.1 \\
\pm 0.4\end{array}$ & $\begin{array}{l}0.45 \\
\pm 0.27\end{array}$ & $\begin{array}{l}0.54 \\
\pm 0.07\end{array}$ & $\begin{array}{l}0.63 \\
\pm 0.06\end{array}$ & $\begin{array}{l}0.02 \\
\pm 0.01\end{array}$ \\
\hline
\end{tabular}


Table 4.6: Radium activities $\left(\mathrm{Bq} \mathrm{kg}^{-1}\right)$ and their ratios measured in August 1986 from a brine sample collected from GB2 production well. Reported errors are $2 \sigma$ uncertainties. Data are provided by EnBW and corrected to the sitespecific fluid density of $\varrho_{b}=1023.24 \mathrm{~kg} / \mathrm{m}^{3}$.

\begin{tabular}{lll}
\hline & Radium & Activity in brine \\
\hline Ra isotopes & ${ }^{224} \mathrm{Ra}$ & $9.1 \pm 1.6$ \\
& ${ }^{228} \mathrm{Ra}$ & $12.1 \pm 1.1$ \\
& ${ }^{226} \mathrm{Ra}$ & $22.9 \pm 1.8$ \\
\hline Isotopic ratio & ${ }^{224} \mathrm{Ra} /{ }^{228} \mathrm{Ra}$ & $0.75 \pm 0.20$ \\
& ${ }^{228} \mathrm{Ra} /{ }^{226} \mathrm{Ra}$ & $0.53 \pm 0.09$ \\
\hline
\end{tabular}

\subsection{Modelling the disequilibria in water-rock systems}

Water-rock interaction processes in the Bruchsal geothermal reservoir were mathematically modelled based on $\mathrm{Ku}$ et al. (1992). This model allows for physico-chemical reactions as well as advective transport. The model is robust in terms of input parameters and focuses on the simulation of the behavior of radium in the rock-brine environment. Employment of the various radium isotopes provides a way of quantifying relevant parameters of the water-rock system due to their wide range of half-lives.

Theoretically, the model can be applied to all elements with numerous instable isotopes such as $\mathrm{U}$ $\left.{ }^{238} \mathrm{U},{ }^{235} \mathrm{U},{ }^{234} \mathrm{U}\right)$ and $\mathrm{Th}\left({ }^{234} \mathrm{Th},{ }^{232} \mathrm{Th},{ }^{231} \mathrm{Th},{ }^{230} \mathrm{Th},{ }^{228} \mathrm{Th},{ }^{227} \mathrm{Th}\right)$. However, Th-U isotopes are not used in this study due to their poor solubility in the Bruchsal brine and the resulting lack of data.

\subsubsection{Model assumptions suggested by Ku et al. (1992)}

The processes of sorption-desorption and dissolution-precipitation of radionuclides are determined by reaction kinetics. Ku et al. (1992) subdivided three "pools" in which radionuclides can reside: the dissolved, adsorbed, and solid pool.

Fig. 4.5 depicts a schematic representation of the conceptual model including water-rock interaction processes and the three different "pools". Model parameters are listed in Table 4.7. 


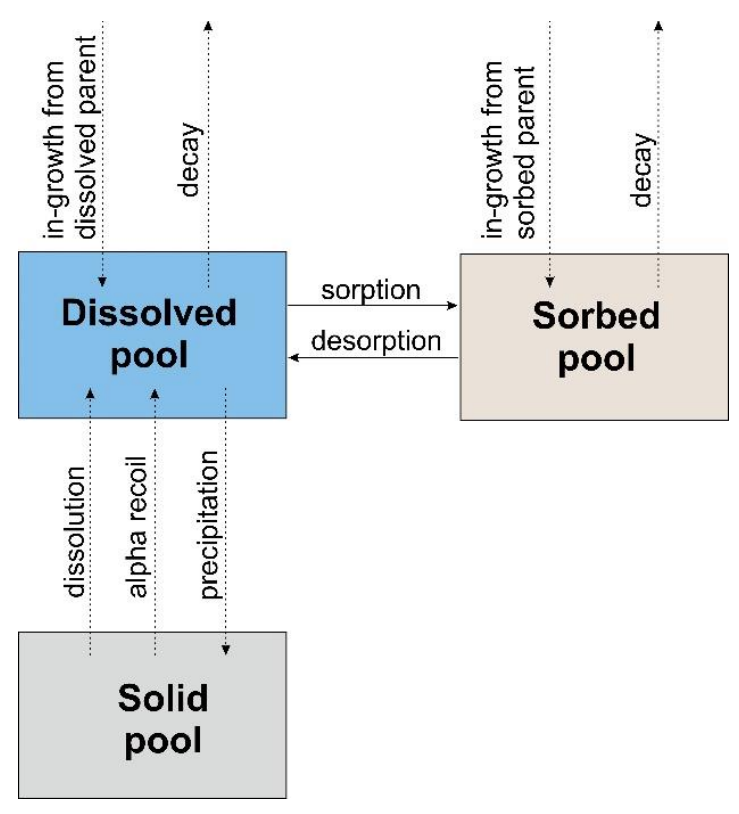

Fig. 4.5: Schematic representation of the water-rock interaction model suggested by Ku et al. (1992). Reversible and irreversible processes are indicated by arrows with solid and dashed lines. The figure is reworked in accordance to Ku et al. (1998).

Table 4.7: Parameters used in the water-rock interaction model.

\begin{tabular}{lll}
\hline Symbol & Parameter & SI units \\
\hline$A$ & Specific activity of a dissolved radionuclide & $\mathrm{Bq} \mathrm{kg}^{-1}$ \\
$A^{*}$ & Specific activity of a dissolved radionuclide originated from diffusional & $\mathrm{Bq} \mathrm{kg}-1$ \\
$A_{a}$ & flux & $\mathrm{Bq} \mathrm{kg}^{-1}$ \\
$\bar{A}$ & Specific activity of an adsorbed radionuclide & $\mathrm{Bq} \mathrm{kg}^{-1}$ \\
$C$ & Specific activity of a radionuclide in solids & atoms kg-1 \\
$C^{i}$ & Concentration of a dissolved radionuclide & atoms kg-1 $^{-1}$ \\
$D_{m}$ & Initial concentration of a dissolved radionuclide & $\mathrm{m}^{2} \mathrm{~s}^{-1}$ \\
$F$ & Diffusion coefficient of a radionuclide & $\mathrm{Bq} \mathrm{m}^{-2}$ \\
$e_{R a}$ & Diffusional flux of a nuclide into larger fractures & - \\
$\varepsilon_{i}$ & Radium recoil efficiency relative to ${ }^{222 \mathrm{Rn}}$ & - \\
$\phi$ & Recoil efficiency for nuclide $i$ & - \\
$k_{1}$ & Porosity of the aquifer & $\mathrm{s}^{-1}$ \\
$k_{2}$ & First-order adsorption rate constant & $\mathrm{s}^{-1}$ \\
$k_{p}$ & First-order desorption rate constant & $\mathrm{s}^{-1}$ \\
$k_{w}$ & First-order precipitation rate constant & $\mathrm{s}^{-1}$ \\
$K$ & First-order dissolution rate constant & -
\end{tabular}




\begin{tabular}{|c|c|c|}
\hline Symbol & Parameter & SI units \\
\hline$K_{d}$ & Distribution coefficient & $\mathrm{L} \mathrm{kg}^{-1}$ \\
\hline$\lambda$ & Radioactive decay constant of radionuclide & $\mathrm{s}^{-1}$ \\
\hline$M_{b}$ & Mass of brine & $\mathrm{kg} \mathrm{m}^{-3}$ \\
\hline$M_{r}$ & Mass of rock & $\mathrm{kg} \mathrm{m}^{-3}$ \\
\hline$P$ & Supply rate of radionuclide to brine & $\mathrm{Bq} \mathrm{kg}{ }^{-1}$ \\
\hline$P_{d}$ & Supply rate of radionuclide to brine by desorption & $\mathrm{Bq} \mathrm{kg}^{-1}$ \\
\hline$P_{r}$ & Supply rate of radionuclide to brine by alpha recoil & $\mathrm{Bq} \mathrm{kg}{ }^{-1}$ \\
\hline$P_{r}^{*}$ & $\begin{array}{l}\text { Supply rate of radionuclide to brine by alpha recoil in consideration of } \\
\text { diffusional flux }\end{array}$ & $\mathrm{Bq} \mathrm{kg}-1$ \\
\hline$P_{w}$ & Supply rate of radionuclide to brine by dissolution process & $\mathrm{Bq} \mathrm{kg}^{-1}$ \\
\hline$Q$ & Supply rate of radionuclide by water flow & $\mathrm{Bq} \mathrm{kg}{ }^{-1}$ \\
\hline$r$ & Recoil distance & $\mathrm{m}$ \\
\hline$R_{f}$ & Retardation factor due to adsorption and desorption & - \\
\hline$R_{f}^{*}$ & $\begin{array}{l}\text { Retardation factor due to precipitation as well as adsorption and desorp- } \\
\text { tion }\end{array}$ & - \\
\hline$\rho_{b}$ & Fluid density & $\mathrm{kg} \mathrm{m}^{-3}$ \\
\hline$\rho_{s}$ & Density of aquifer solid & $\mathrm{kg} \mathrm{m}^{-3}$ \\
\hline$S$ & $\begin{array}{l}\text { Surface area of solids, expressed as area of solid per volume of fluid con- } \\
\text { tacting the solids }\end{array}$ & $\mathrm{m}^{2} \mathrm{~m}^{-3}$ \\
\hline$S_{a}$ & Scavenging rate of radionuclide from brine by adsorption to rock surfaces & $\mathrm{Bq} \mathrm{kg}{ }^{-1}$ \\
\hline$S_{p}$ & $\begin{array}{l}\text { Scavenging rate of radionuclide from brine by co-precipitation with min- } \\
\text { erals }\end{array}$ & $\mathrm{Bq} \mathrm{kg}{ }^{-1}$ \\
\hline$\tau_{b}$ & Transit time of brine in the aquifer & s \\
\hline$\tau_{p, i}$ & Mean time for nuclide $i$ in solution to precipitate in minerals & s \\
\hline$\tau_{w, i}$ & Mean time for leach nuclide $i$ from solid phases & $\mathrm{s}$ \\
\hline$w_{f}$ & Fracture width & $\mathrm{m}$ \\
\hline$v_{f}$ & Advection velocity of fracture fluid & $\mathrm{m} \mathrm{s}^{-1}$ \\
\hline$x$ & Distance & $\mathrm{m}$ \\
\hline ' & Superscript referring to radioactive parent & - \\
\hline
\end{tabular}

Ku et al. (1992) defined the following model assumptions:

(1) In the dissolved "pool", radionuclides are exchangeable with those in the adsorbed pool, but not with those in the solid "pool".

(2) Transfer of radionuclides between the dissolved and solid "pools" is achieved in particular by dissolution, co-precipitation and alpha recoil. 
(3) Dissolution and precipitation are considered irreversible because dissolved nuclides have limited and very slow communication with the solid "pool" which is located further inside the rock matrix.

(4) Alpha-recoil input from the adsorbed and dissolved "pools" to the solid "pool" is negligible.

(5) Distributions of radionuclides in solid, adsorbed, and dissolved "pools" remain stationary.

\subsubsection{Governing equations}

Based on mass balance, the activity of a given radionuclide dissolved in a volume of brine with a constant density can be expressed as (Lou et al., 2000):

$$
Q+P_{r}+P_{w}+R_{f}^{\prime} A^{\prime}=k_{p} C+R_{f} A
$$

where

Q Supply rate by water flow, atoms $\mathrm{kg}^{-1} \mathrm{~s}^{-1}$

$P_{w} \quad$ Supply rate of radionuclide to fluid by dissolution, atoms $\mathrm{kg}^{-1} \mathrm{~s}^{-1}$

$P_{r} \quad$ Supply rate of radionuclide to fluid by alpha recoil, atoms $\mathrm{kg}^{-1} \mathrm{~s}^{-1}$

$R_{f} \quad$ Retardation factor due to adsorption and desorption; dimensionless

$A \quad$ Radionuclide activity in brine, atoms $\mathrm{kg}^{-1} \mathrm{~s}^{-1}(=\lambda C)$

$k_{p} \quad$ First-order precipitation rate constant; $\mathrm{s}^{-1}$

C Radionuclide concentration in brine, atoms $\mathrm{kg}^{-1}$

Superscript referring to the radioactive parent

The retardation factor $R_{f}$ is formulated as follows (Krishnaswami et al., 1982)

$$
R_{f}=1+K=1+\frac{k_{1}}{k_{2}+\lambda}
$$

where

$\lambda \quad$ radioactive decay constant of radionuclide, $\mathrm{s}^{-1}$ 
$K$

dimensionless distribution coefficient

$k_{1} \quad$ first-order adsorption rate constant, $\mathrm{s}^{-1}$

$k_{2} \quad$ first-order desorption rate constant, $\mathrm{s}^{-1}$

For all radium isotopes whose thorium parents are quite insoluble in the geothermal fluid, $R_{f}^{\prime} A^{\prime}$ is negligible

$$
Q+P_{r}+P_{w}=k_{p} C+R_{f} A
$$

Furthermore, processes of dissolution and precipitation will not influence the activity of shortlived radionuclides and thus, by setting $P_{W}=0$ and $k_{p} C=0$, Eqn. (4.16) may be simplified to

$$
Q+P_{r}=R_{f} A
$$

where

$$
Q=\frac{\left(C^{i}-C\right)}{\tau_{b}}
$$

$C^{i}$ and $C$ are initial and measured concentrations, respectively, and $\tau_{b}$ is the transit time of brine in the aquifer. Positive or negative values of $Q$ denote net gain or loss due to fluid flow, i.e. advective transport (Luo et al., 2000).

\subsubsection{Alpha recoil}

Alpha recoil describes a process in which a radioactive daughter is mobilized from its initial position by the energy of an alpha decay (Sun and Semkow, 1998). During the decay, an atomic nucleus emits an alpha particle. The released ionizing radiation has an energy content of 4-6 MeV. Because of the law of conservation of momentum, the emitted alpha particle and recoiling nucleus will each have a well-defined energy after the decay. Because of its smaller mass, most of the kinetic energy is transferred to the alpha particle. The recoiling nucleus will have a kinetic energy in the order of $100 \mathrm{keV}$ (Sun and Semkow, 1998). Nevertheless, the energy transfer to the decay product is high enough to shift atoms that are close to the mineral surface out of the mineral grain into the pore space (Fig. 4.6). The probability of recoiling out of mineral grains depends on the isotope recoil distance and the number of previous alpha decays. While ${ }^{228} \mathrm{Ra}$ is directly formed by the decay of ${ }^{228} \mathrm{Th},{ }^{226} \mathrm{Ra}$ is formed by three alpha decays of ${ }^{238} \mathrm{U}$. Thus, the probability of ${ }^{226} \mathrm{Ra}$ to end up in the fluid by alpha recoil is significantly greater than the probability for ${ }^{228} \mathrm{Ra}$ due to the greater amount of the three alpha energies. 


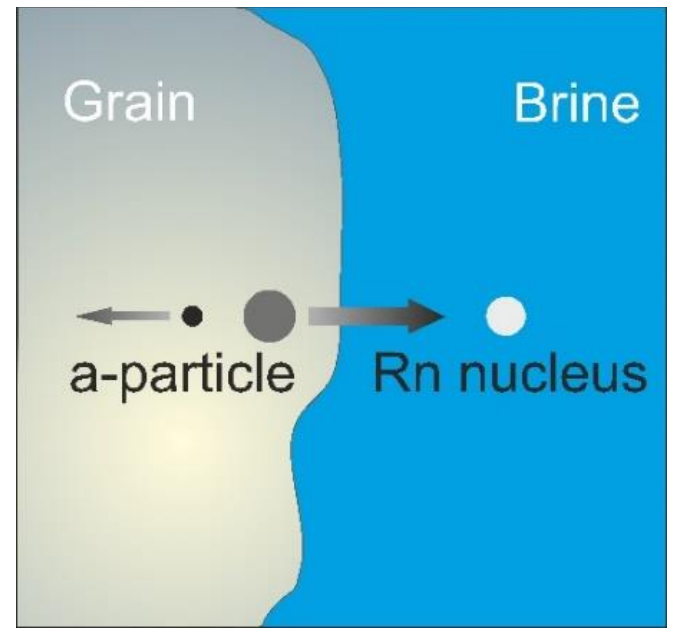

Fig. 4.6: Schematic diagram of alpha recoil mechanism. Mobilization of a radioactive daughter from its initial position by the energy of an alpha decay. Arrow size reflects energy content.

Alpha recoil supply rates $\left(P_{r}\right)$ for radium isotopes can be estimated from the activities of the decay series progenitors ${ }^{238} \mathrm{U},{ }^{235} \mathrm{U}$ and $\left.{ }^{232} \mathrm{Th}\right)$ in the adjoining rock expressed as (e.g. Kigoshi, 1971):

$$
P_{r}=\bar{A}^{\prime} \varepsilon_{i} r S \rho_{S}
$$

where $\overline{A^{\prime}}$ is the parent activity in solids (atoms $\left.\mathrm{kg}^{-1} \mathrm{~s}^{-1}\right), r$ is the recoil distance $(\AA), \varepsilon_{i}$ is the recoil efficiency for nuclide $i$ O, $S$ is the surface area of solids, expressed as area of solid per volume of fluid contacting the solids $\left(\mathrm{m}^{2} \mathrm{~m}^{-3}\right)$ also called flow wetted surface and $\rho_{s}$ is the density of solid (kg $\left.\mathrm{m}^{-3}\right)$.

For the geothermal site in Bruchsal, the average progenitor activities $\left(\overline{A^{\prime}}\right)$ in the reservoir section are ${ }^{238} \mathrm{U}=18.0 \mathrm{~Bq} \mathrm{~kg}{ }^{-1},{ }^{232} \mathrm{Th}=17.0 \mathrm{~Bq} \mathrm{~kg}$-1 and ${ }^{235} \mathrm{U}^{2}=0.8 \mathrm{~Bq} \mathrm{~kg}^{-1}$ (Kölbel et al., 2020). Surface area per mass is about $2000 \mathrm{~m}^{2} \mathrm{~kg}^{-1}$ for the Permo-Triassic sandstones (Heap et al., 2019). This corresponds to a flow wetted surface of $1.0 \times 10^{8} \mathrm{~m}^{2} \mathrm{~m}^{-3}$ based on a porosity of 0.05 . Sun and Semkow (1998) published data of Ra recoil distances of $800 \AA$ in the surface zone of quartz obtained from Monte Carlo simulations (Table 4.8). Since recoil efficiencies for radium isotopes are not that easy to determine, $\varepsilon_{i}$ is part of the discussion.

\subsubsection{Retardation factor and distribution coefficient}

The retardation factor $R_{f}$ describes the flow rate of the fluid relative to the rate of migration of a radionuclide in the flow ( $\mathrm{Ku}$ et al., 1992). The separation between the adsorbed and dissolved nuclides through chemical exchanges might be stated by the dimensionless distribution coefficient K (Krishnaswami et al., 1987): 
Table 4.8: Average and maximum recoil distances of radium isotopes in quartz which is chosen as the host due to the mineralogical composition of the aquifer. Data originate from Sun and Semkow (1998).

\begin{tabular}{ccc}
\hline Decay & Average distance $(\AA)$ & Maximum distance $(\AA)$ \\
\hline${ }^{230} \mathrm{Th} \rightarrow{ }^{226} \mathrm{Ra}$ & 370.3 & 663.2 \\
${ }^{232} \mathrm{Th} \rightarrow{ }^{228} \mathrm{Ra}$ & 331.3 & 609.8 \\
${ }^{228} \mathrm{Th} \rightarrow{ }^{224} \mathrm{Ra}$ & 406.6 & 763.3 \\
\hline
\end{tabular}

$$
K=\frac{A_{a}}{A}
$$

Where $A_{a}$ is the activity of an adsorbed radionuclide (atoms per equivalent fluid-volume) and $A$ is the radionuclide activity dissolved in solution (atoms per fluid-volume).

Since distribution coefficients are usually determined by adsorption-desorption experiments in the laboratory and therefore, expressed in units of volume per mass, $K$ can be derived from

$$
K=K_{d}\left[\frac{\rho_{s}(1-\phi)}{\phi}\right]
$$

where

$K_{d} \quad$ distribution coefficient, volume mass $^{-1}$;

$\rho_{s} \quad$ density of aquifer solids, mass solid volume ${ }^{-1}$;

$\phi \quad$ porosity of the aquifer, dimensionless.

Krishnaswami et al. (1992) expressed $R_{f}$ and $K$ in terms of adsorption and desorption rate constants, $k_{1}$ and $k_{2}$ :

$$
\begin{aligned}
& R_{f}=1+K=\frac{k_{1}+k_{2}+\lambda}{k_{2}+\lambda}=\frac{1}{\Omega} \\
& k_{1}=\frac{\left(\lambda_{i}-\lambda_{j}\right)\left(1-\Omega_{i}\right)\left(1-\Omega_{j}\right)}{\left(\Omega_{i}-\Omega_{j}\right)} \\
& k_{2}=\frac{\Omega_{i} \Omega_{j}\left(\lambda_{j}-\lambda_{i}\right)+\lambda_{i} \Omega_{j}-\lambda_{j} \Omega_{i}}{\left(\Omega_{i}-\Omega_{j}\right)}
\end{aligned}
$$

where $i$ and $j$ refer to two isotopes of the same element and $\Omega$ is the ratio of the activity of a nuclide 
in solution, $\lambda C$, to its rate of production $P$ :

$$
\Omega=\frac{\lambda C}{P}
$$

Since these authors did not consider dissolution processes, the supply rate $P$ only includes recoil $\left(P_{r}\right)$ and in-situ production $\left(\lambda^{\prime} C\right)$ and thus, this model is only valid for radionuclides with half-lives less than 10 years (Appendix 1, Krishnaswami et al., 1982).

\subsubsection{Precipitation and dissolution}

The role of precipitation and dissolution processes becomes more apparent for longer-lived radionuclides. Rate constants for precipitation and dissolution may be calculated by the mass balances of radium isotopes.

Hammond et al. (1988) defined radium input to brine by dissolution processes, $P_{w}\left(\right.$ atoms s $\mathrm{kg}^{-1}$ ), by the following equation:

$$
P_{w}=\frac{k_{w} \bar{A}}{\lambda}
$$

where $\bar{A}$ is the radium activity in rocks (atoms $\mathrm{kg}^{-1} \mathrm{~s}^{-1}$ ), $k_{m}$ is the first-order rate constant for dissolution $\left(\mathrm{s}^{-1}\right)$, and $\lambda$ is the decay constant of the respective radium isotope $\left(\mathrm{s}^{-1}\right)$.

The converse process, radium co-precipitation with minerals, $S_{p}$ (atoms s${ }^{-1} \mathrm{~kg}^{-1}$ ), can be expressed by

$$
S_{p}=k_{p} C
$$

where $k_{p}$ is the first-order rate constant for precipitation $\left(\mathrm{s}^{-1}\right)$ and $C$ the concentration in brine $\left(\right.$ atoms $\left.\mathrm{kg}^{-1}\right)$.

\subsection{Model performance}

The introduced mass balance approach was modeled using Mathcad ${ }^{\circledR}$, a numerical software with computer algebra system (CAS) capabilities. In order to check the CAS approach, MIN3P, a multicomponent reactive transport code, was employed (Mayer at al., 2002).

MIN3P is a general-purpose flow and reactive transport code for variably saturated media providing a high degree of flexibility with respect to the definition of the reaction network. Advective- 
diffusive transport in the water phase and diffusive transport in the gas phase are included. Equilibrium reactions considered are aqueous complexation, gas partitioning between phases, oxidation-reduction, ion exchange, and surface complexation. The reaction network is designed to handle kinetically controlled intra-aqueous and dissolution-precipitation reactions.

All reactions can be defined through databases of MINTEQA2 (Allison at al., 1991) and PHREEQC2 (Parkhurst and Appelo, 1999).

Table 4.9 lists the set of data that were used for the comparison. Input parameters correspond to the physical parameters of the Bruchsal site which are required for reactive transport modelling (Joachim et al., 1987).

Table 4.9: Site specific input parameter used for the model comparison between Mathcad® and MIN3P. Data from Joachim et al. (1987).

\begin{tabular}{lccc}
\hline Parameter & Symbol & Unit & Value \\
\hline Porosity & $\phi$ & - & 0.05 \\
Hydraulic conductivity & $k_{f}$ & $\mathrm{~m} \mathrm{~s}^{-1}$ & $4.3 \times 10^{-6}$ \\
Darcy velocity & $v_{\mathrm{f}}$ & $\mathrm{m} \mathrm{s}^{-1}$ & $3.2 \times 10^{-8}$ \\
Longitudinal dispersion & $\alpha_{L}$ & $\mathrm{~m}$ & 0.1 \\
\hline
\end{tabular}

\subsubsection{Recoil mechanism}

Since MIN3P treats the recoil mechanism as an intra-aqueous reaction, recoil supply rates were specified in the respective database file. Assuming that short-lived nuclides are mainly controlled by alpha recoil, ${ }^{224} \mathrm{Ra}$ was used as an example to test the accuracy of the recoil term. Fig. 4.7 illustrates the results of the comparison between MIN3P and Mathcad ${ }^{\circledR}$.

\subsubsection{Kinetic approach for solid solutions}

Co-precipitation of radium with barite is an important process affecting radionuclide reactive transport in rock formations. It is generally described using a solid solution model (Parkhurst and Appelo, 2013). Commonly, geochemical equilibria are quantified by the law of mass action. A suitable example is given by the reaction of two components $A$ and $B$ with their stoichiometric constants $a$ and $b$. Considering $A$ and $B$ as the aqueous components and $A B$ as the solid phase components $(a A+b B \rightleftharpoons A B)$ leads to the equilibrium constant 


$$
K_{e q}=\frac{\{A\}^{a}\{B\}^{b}}{\{A B\}}
$$

where brackets \{\} represent the activity of the components. For the condition of a homogeneous solid, its activity is assumed unity, hence Eqn. (4.27) is simplified to $K_{e q}=\{A\}^{a}\{B\}^{b}$, generally known as the solubility product of $A B$.

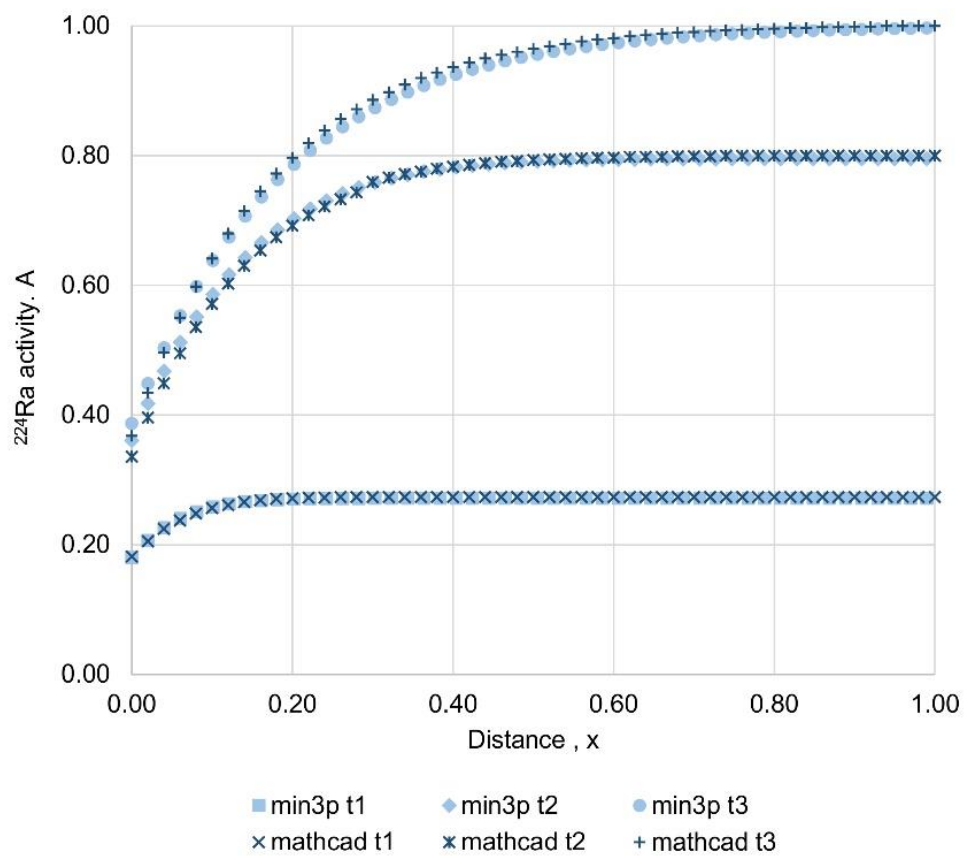

Fig. 4.7: Comparison of MIN3P and CAS model for one dimensional transport of ${ }^{224} \mathrm{Ra}$ along a flow line, calculated at different half-lives $\left(\mathrm{t}_{1}=0.5 \mathrm{t}_{1 / 2} ; \mathrm{t}_{2}=2 \mathrm{t}_{1 / 2} ; \mathrm{t}_{3}=45 \mathrm{t}_{1 / 2}\right)$.

For a solid solution, i.e. a mixture of several constituents, this simplification does not hold. The specific solid phase activity becomes dependent on its mole fraction $X_{i}$, yielding a set of concurrent equations. For simplicity, we neglect a potential non-ideality within the solid mixture, which would require the introduction of non-unity activity coefficients. It might as well be noted that often $K_{e q}$ is defined in a reciprocal way. In the given background the two reactions are:

$$
\begin{aligned}
& \mathrm{Ba}^{2+} \mathrm{SO}_{4}^{2-} \rightleftharpoons \mathrm{BaSO}_{4} \\
& \mathrm{Ra}^{2+} \mathrm{SO}_{4}^{2-} \rightleftharpoons \mathrm{RaSO}_{4}
\end{aligned}
$$

with the associated solubility products

$$
K_{B a}=\frac{\left\{\mathrm{Ba}^{2+}\right\}\left\{\mathrm{SO}_{4}^{2-}\right\}}{X_{\text {BaSO4 }}}
$$




$$
K_{R a}=\frac{\left\{\mathrm{Ra}^{2+}\right\}\left\{\mathrm{SO}_{4}^{2-}\right\}}{X_{\text {RaSO }}}
$$

The equilibrated solution to this set of equations is a bit more cumbersome to achieve and the reader may be referred to e.g. Rodriguez-Galan and Prieto (2018). However, if the equilibrium can be approached in a kinetic simulation, the forward and backward reactions can be separated and make use of Lasaga's principle of detailed balancing (Lasaga, 1998) to obtain a considerably more straightforward procedure:

$$
\begin{aligned}
& \mathrm{Ba}^{2+} \mathrm{SO}_{4}^{2-} \rightarrow \mathrm{BaSO}_{4} \\
& \mathrm{BaSO}_{4} \rightarrow \mathrm{Ba}^{2+} \mathrm{SO}_{4}^{2-}
\end{aligned}
$$

and

$$
\begin{aligned}
& \mathrm{Ra}^{2+} \mathrm{SO}_{4}^{2-} \rightarrow \mathrm{RaSO}_{4} \\
& \mathrm{RaSO}_{4} \rightarrow \mathrm{Ra}^{2+} \mathrm{SO}_{4}^{2-}
\end{aligned}
$$

with the kinetic rate expressions

$$
\begin{aligned}
& R_{+}=k_{+}\left\{\mathrm{Ba}^{2+}\right\}\left\{\mathrm{SO}_{4}^{2-}\right\} \\
& R_{-}=k_{-} X_{\text {BaSO } 4}
\end{aligned}
$$

in terms of the total reaction

$$
R_{t}=k_{+}\left\{\mathrm{Ba}^{2+}\right\}\left\{\mathrm{SO}_{4}^{2-}\right\}-k_{-} X_{\mathrm{BaSO} 4}=k_{+}\left\{\mathrm{Ba}^{2+}\right\}\left\{\mathrm{SO}_{4}^{2-}\right\}\left(1-\frac{I A P}{K_{e q}}\right)
$$

Resulting in the forward activity product time an affinity term $\left(1-L A P / K_{\text {eq }}\right)$, of which $L A P$ is the complete ion activity product at current condition $X_{\mathrm{BaSO} 4} /\left(\left\{\mathrm{Ba}^{2+}\right\}\left\{\mathrm{SO}_{4}{ }^{2-}\right\}\right)$, and $K_{e q}$ is its counterpart for equilibrium conditions.

Assuming that radium is always present in concentrations orders of magnitude lower compared to barium, so that the solid fraction only remains relevant for $\mathrm{Ra}^{2+}$, it follows

$$
\begin{aligned}
& X_{\mathrm{BaSO}_{4}}=\frac{\left\{\mathrm{BaSO}_{4}\right\}}{\left\{\mathrm{RaSO}_{4}\right\}+\left\{\mathrm{BaSO}_{4}\right\}} \cong 1 \quad \text { as }\{\mathrm{Ba}\} \gg\{\mathrm{Ra}\} \\
& X_{\mathrm{RaSO} 4}=\frac{\left\{\mathrm{RaSO}_{4}\right\}}{\left\{\mathrm{RaSO}_{4}\right\}+\left\{\mathrm{BaSO}_{4}\right\}} \cong \frac{\left\{\mathrm{RaSO}_{4}\right\}}{\left\{\mathrm{BaSO}_{4}\right\}}
\end{aligned}
$$


That allows treating barite dissolution/precipitation as a kinetic reversible process as given in the database, independent of the radium co-precipitation process, leaving the two kinetic rate expressions $(k 1+$ and $k 1-)$ for radium left to solve for separately, with lumped rate constants $k_{+} / k_{\text {: }}$

$$
\begin{aligned}
& R_{+}=\frac{k_{+}}{k_{-}}\left\{\mathrm{Ba}^{2+}\right\}\left\{\mathrm{SO}_{4}^{2-}\right\} \\
& R_{-}=X_{\text {RaSO } 4}
\end{aligned}
$$

MIN3P's solid solution term was checked based on literature data (Grandia et al., 2008) resulting in $(\mathrm{Ra}, \mathrm{Ba}) \mathrm{SO}_{4}$ equilibrium lines which have a similar slope for varying natural $\mathrm{Ra}-\mathrm{Ba}$ trends (Fig. 4.8). The equilibrium line for the Bruchsal site shows the same trend as model derived equilibrium lines from Grandia et al. (2008). Start and end point of the solid line is defined by the fluid-specific barium concentration and ${ }^{226} \mathrm{R}$ a activity in brine.

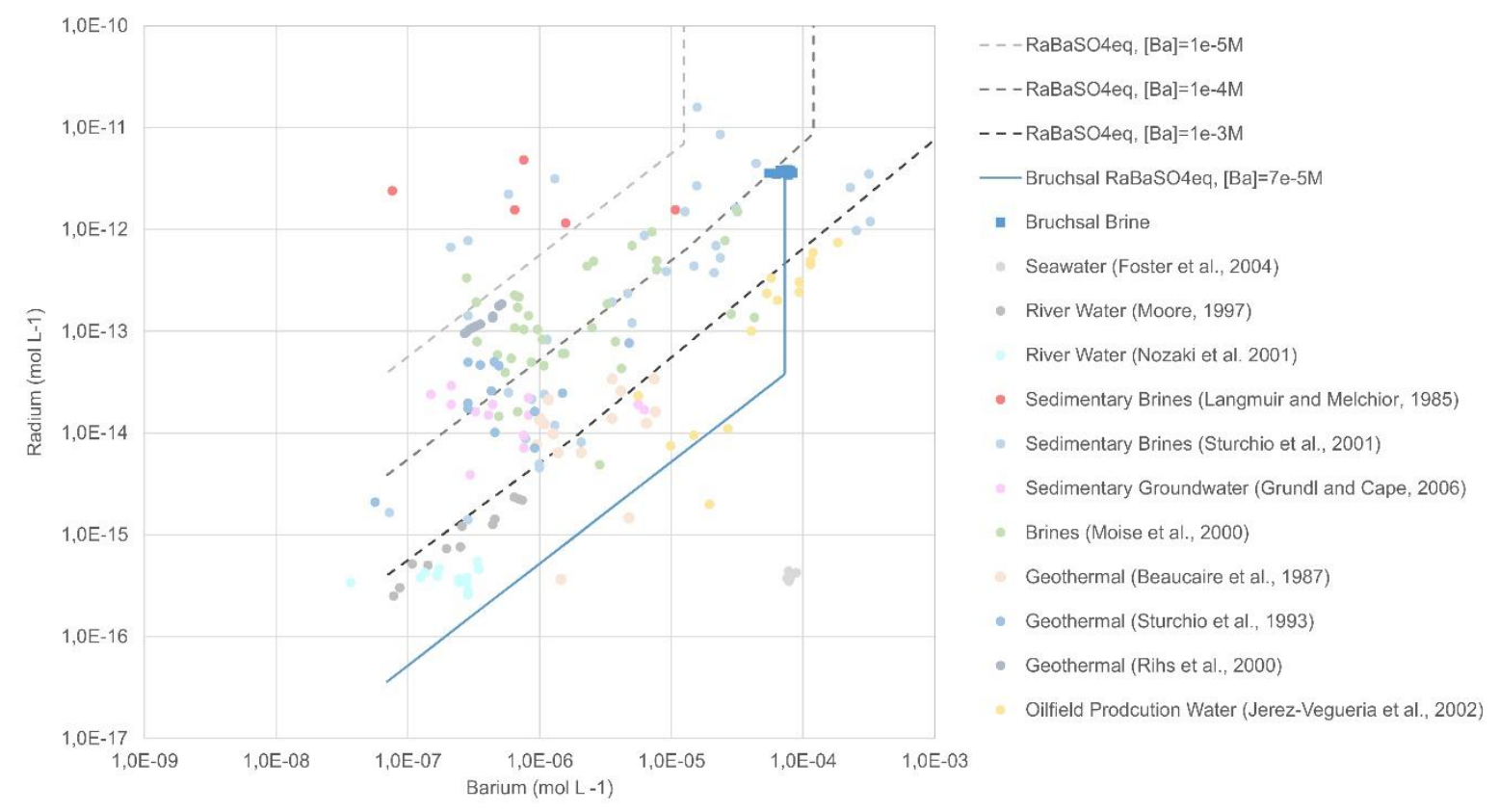

Fig. 4.8: Re-calculation of radium co-precipitation with barite from literature data (Grandia et al., 2008). Calculations result in $(\mathrm{Ra}, \mathrm{Ba}) \mathrm{SO}_{4}$ equilibrium lines having a similar slope for varying natural $\mathrm{Ra}-\mathrm{Ba}$ trends.

\subsection{Discussion}

Modelling the physico-chemical mechanisms for radionuclide supply and removal at the geothermal site in Bruchsal are based on the following assumptions: An isotropic system is assumed in 
which a large conductive fracture of width $w_{f}$ and height $h_{f}$ are intersected by microfractures. These microfractures are part of the rock matrix with a very low hydraulic conductivity and thus, water flow is assumed to take place only in the fracture.

The flow rate is parallel to the fracture orientation with a fracture length $L_{f}$ that coincides with the principal direction of groundwater flow. The fracture dimensions are assumed to be large relative to their aperture. Water-rock interactions take place in both regions, in the matrix blocks and in the fracture. However, the latter has a relatively low water-rock interaction rate due to their differences in surface area to fluid ratio. A schematic overview about the Bruchsal reservoir is shown in Fig. 4.9.

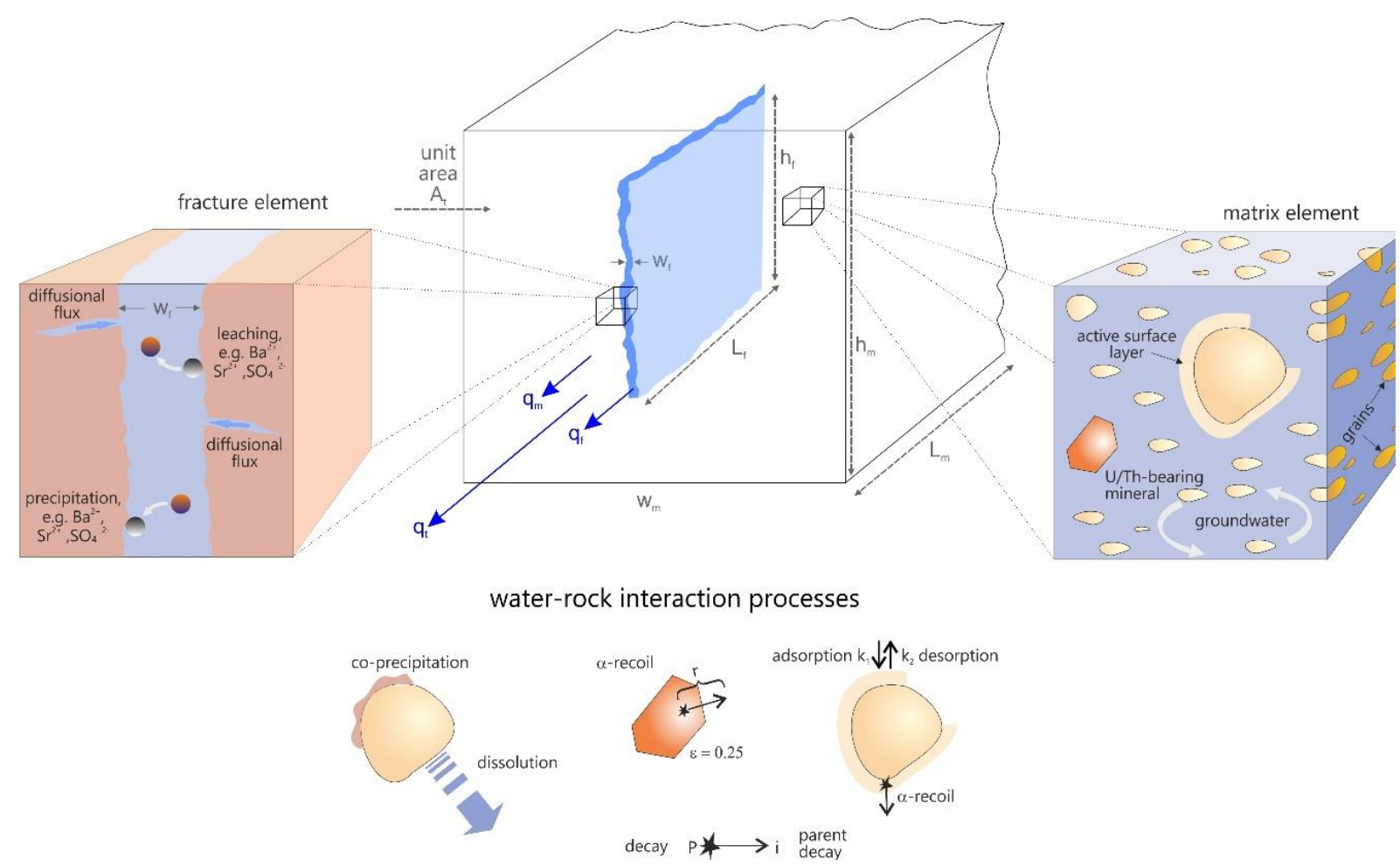

Fig. 4.9: Conceptual model for the Bruchsal geothermal site: Fracture-dominated radionuclide transport within the reservoir section in consideration of the physico-chemical water-rock interactions (adsorption-desorption, dissolutionprecipitation, alpha recoil) which take place to varying extent in both, the fracture and matrix element (Figure extended after Tricca et al., 2000).

Since water-rock interaction rates occur at different timescales, their impact on U-Th series radionuclides varies depending on their half-lives. While mineral dissolution (leaching) mainly affects long-lived radionuclides, the physical process of alpha recoil is mainly associated with short-lived nuclides (cf. Eqn. (4.17)).

Assuming that large fractures channel the geothermal brine and alpha recoil directly into fractures 
is the only process adding radium into solution, the observed ${ }^{224} \mathrm{Ra} /{ }^{228} \mathrm{Ra}$ ratios should be greater than or equal to those in the rock material. However, ratios observed in the Bruchsal brine are lower $\left(\right.$ mean $\left.{ }^{224} \mathrm{Ra} /{ }^{228} \mathrm{Ra}=0.65\right)$, indicating that alpha recoil depends on half-lives of the respective radionuclides.

\subsubsection{Alpha recoil supply rates and recoil efficiencies}

Modeling the radium supply from recoil $\left(P_{r}\right)$, Eqn. (4.19) is applied to the site-specific input parameter. Since the recoil efficiency is hard to determine, it might be estimated from ${ }^{222} \mathrm{Rn}$ activity in brine. Since ${ }^{222} \mathrm{Rn}$ is an inert gas, it is entirely dissolved and can therefore be measured directly. Its production is from alpha recoil of ${ }^{226} \mathrm{Ra}$ that is within a recoil distance of $\sim 40 \mathrm{~nm}$ of mineral surfaces as well as from the decay of the dissolved parent ${ }^{226} \mathrm{Ra}$ in brine. Therefore, mass balance for ${ }^{222} \mathrm{Rn}$ can be expressed as follows:

$$
A_{222 R n}=P_{r, 222 R n}+R_{f, 226 R a} A_{226 R a}
$$

The fraction of ${ }^{222} \mathrm{Rn}$ atoms that is produced by alpha recoil to its total fluid activity describes its recoil efficiency. Lou et al. (2000) proposed the following equation for calculating the ${ }^{222} \mathrm{Rn}$ recoil efficiency

$$
\varepsilon_{222}=\frac{P_{r, 222 R n}}{A_{222 R n}}=\frac{1}{1+\left(\left(\overline{\overline{A_{238 U}}}\right) \cdot\left(\frac{A_{224 R a}}{\overline{A_{232 T h}}}-1\right) \cdot\left(\frac{A_{228 R a}}{A_{226 R a}}\right)\right)^{-1}}
$$

assuming that ratio of alpha recoil supply for ${ }^{222} \mathrm{Rn}$ and ${ }^{224} \mathrm{Ra}$ equals the ${ }^{238} \mathrm{U} /{ }^{232} \mathrm{Th}$ activity ratio in rocks. According to that ${ }^{222} \mathrm{Rn}$ recoil efficiency $\left(\varepsilon_{222}\right)$ is ca. $23 \%$ at the Bruchsal geothermal site, suggesting that the primary source of dissolved ${ }^{222} \mathrm{Rn}$ is ${ }^{226} \mathrm{Ra}$ decay dissolved in brine (rather than the ${ }^{226} \mathrm{Ra}$ decay in the solid phase).

Krishnaswami et al. (1982) used ${ }^{222} \mathrm{Rn}$ to normalize recoil efficiency. They defined the recoil efficiency of nuclide $i$ relative to the ${ }^{222} \mathrm{Rn}$ efficiency as a function of (a) its position in the decay chain and (b) on the adsorption behavior of its progenitor. An example is given for the daughter-parent couple of ${ }^{224} \mathrm{Ra}-{ }^{228} \mathrm{Ra}$. Since both isotopes are members of the ${ }^{232} \mathrm{Th}$ decay chain, ${ }^{224} \mathrm{Ra}$ is closely related to ${ }^{228} \mathrm{Ra}$.

However, while ${ }^{228} \mathrm{Ra}$ is generated by a single alpha decay, ${ }^{224} \mathrm{Ra}$ is generated by two alpha decays (cf. Fig. 4.1). Thus, for ${ }^{224} \mathrm{Ra}$ the probability of recoiling into water is significantly larger than that for ${ }^{228} \mathrm{Ra}$, resulting in a higher recoil efficiency for ${ }^{224} \mathrm{R}$ a relative to ${ }^{228} \mathrm{Ra}$. Table 4.10 lists values of recoil efficiencies for radium as well as the recoil supply rates calculated from Eqn. (4.19). 
Table 4.10: Recoil efficiency for radium ( $\left.e_{\mathrm{R} a}\right)$ relative to ${ }^{222} \mathrm{Rn}$ (data originate from Krishnaswami et al., 1982) as well as model derived rates of alpha recoil supply $\left(P_{r}\right)$ for the geothermal system in Bruchsal.

\begin{tabular}{lll}
\hline Decay & $e_{\mathrm{Ra}} \mathrm{O}$ & $P_{r}\left({\left.\operatorname{atoms~} \mathrm{kg}^{-1} \mathrm{~s}^{-1}\right)}^{2}\right.$ \\
\hline${ }^{230} \mathrm{Th} \rightarrow{ }^{226} \mathrm{Ra}$ & 0.961 & 40 \\
${ }^{232} \mathrm{Th} \rightarrow{ }^{228} \mathrm{Ra}$ & 0.769 & 25 \\
${ }^{227} \mathrm{Th} \rightarrow{ }^{223} \mathrm{Ra}$ & 0.961 & 1.7 \\
${ }^{228} \mathrm{Th} \rightarrow{ }^{224} \mathrm{Ra}$ & 0.897 & 41 \\
\hline
\end{tabular}

\subsubsection{Diffusional flux}

Since alpha recoil supply is largest from surface areas with the highest contact areas, the recoil mechanism is most pronounced in the hydraulically inactive rock matrix. Rama and Moore (1984) suggested that diffusion along pore spaces and microfractures is believed to supply the recoiled atoms to the larger fractures where the sampled brine resides.

Here, microfractures serve as diffusion pathways. The flux of radium into the large fracture fluid can be estimated from Eqn. (4.38) as follows (Ku et al., 1992):

$$
F_{R a}=\phi^{*} \sqrt{\frac{D_{m}}{\lambda}}\left(P_{r}+R_{f}^{\prime} A^{\prime}-R_{f} A\right)
$$

where $\Phi^{*}$ is the microfracture porosity, $D_{m}$ is the molecular diffusivity of radium, $\lambda$ is the decay constant, $P_{r}$ is the supply rate from recoil (atoms $\mathrm{kg}^{-1} \mathrm{~s}^{-1}$ ). $R_{f}$ is the retardation factor and $A$ is the activity of dissolved nuclide (atoms $\mathrm{kg}^{-1} \mathrm{~s}^{-1}$ ) with superscript () referring to its radioactive parent.

Porosity is expected to be 0.05 . The molecular diffusivity for radium at $135^{\circ} \mathrm{C}$ is ca. $2.6 \times 10^{-5} \mathrm{~cm}^{2}$ $\mathrm{s}^{-1}$ calculated from the Stokes-Einstein relation (dynamic viscosity $\eta=0.285 \times 10^{-3} \mathrm{~Pa}$ s; hydrodynamic Stokes radius for radium $R_{R a}=3.98 \AA$ ). The term $R_{f}^{\prime} A^{\prime}$ is negligible since thorium progenitors are very insoluble in the geothermal system. The retardation factor of radium is assumed to be 1.3 estimated from the ${ }^{222} \mathrm{Rn} /{ }^{226} \mathrm{Ra}$ brine activity ratio (cf. Table 4.3).

Radium supply from recoil entering the fracture fluid does not only depend on diffusional flux, but also on the fracture width $w_{f}$, expressed as follows

$$
P_{r}^{*}=\frac{2 F_{R a}}{w_{f}}
$$

Applying Eqn. (4.39) to radium with an estimated fracture width $w_{f}$ of $10 \mathrm{~mm}$ (as stated in the GB2 drilling report) results in a significant fractionation of the Ra isotopes (Table 4.12). The diffusional 
flux of radium considering an effective diffusion length $\left(\phi^{*} \sqrt{D_{m} / \lambda}\right)$ is listed in Table 4.11. Since the effective diffusion length depends on the decay constant $\lambda$, it varies between $0.2 \mathrm{~cm}$ for the shortest-lived ${ }^{224} \mathrm{Ra}$ and several tens of centimeter for the longest-lived ${ }^{226} \mathrm{Ra}$ in the geothermal brine. In consequence, the discharge flux density (atoms $\mathrm{cm}^{-2} \mathrm{~s}^{-1}$ ) decreases with increasing decay constants limiting the ${ }^{223} \mathrm{Ra}$ and ${ }^{224} \mathrm{Ra}$ fluid activity (Table 4.11).

Table 4.11: Model derived rates of diffusional flux $F_{R a}$ as a function of the effective diffusion length $\left(\phi^{*} \sqrt{D_{m} / \lambda}\right)$ for radium isotopes.

\begin{tabular}{lll}
\hline Decay & $\phi^{*} \sqrt{D_{m} / \lambda}(\mathrm{cm})$ & $F_{\mathrm{Ra}}\left(\operatorname{atoms~cm}^{-2} \mathrm{~s}^{-1}\right)$ \\
\hline${ }^{230} \mathrm{Th} \rightarrow{ }^{226} \mathrm{Ra}$ & 69 & $1.3 \times 10^{-1}$ \\
${ }^{232} \mathrm{Th} \rightarrow{ }^{228} \mathrm{Ra}$ & 4.2 & $2.9 \times 10^{-2}$ \\
${ }^{227} \mathrm{Th} \rightarrow{ }^{223} \mathrm{Ra}$ & 0.3 & $3.5 \times 10^{-4}$ \\
${ }^{228} \mathrm{Th} \rightarrow{ }^{224} \mathrm{Ra}$ & 0.2 & $5.3 \times 10^{-3}$ \\
\hline
\end{tabular}

Table 4.12: Model derived rate constants of adsorption $\left(k_{1}\right)$ and desorption $\left(k_{2}\right)$; dissolution $\left(k_{w}\right)$ and precipitation $\left(k_{p}\right)$ of radium and the related in-situ retardation factors $\left(R_{r}\right)$ due to sorption processes and $\left(R_{f}^{*}\right)$ due to sorption processes as well as co-precipitation.

\begin{tabular}{|c|c|c|c|c|c|c|}
\hline $\begin{array}{l}\text { Isotopes } \\
\text { of radium }\end{array}$ & $\begin{array}{l}k_{1, R a} \\
\left(\mathrm{~s}^{-1}\right)\end{array}$ & $\begin{array}{l}k_{2, R a} \\
\left(\mathrm{~s}^{-1}\right)\end{array}$ & $\begin{array}{l}R_{f, R a} \\
0\end{array}$ & $\begin{array}{l}k_{n, R a}, \\
\left(\mathrm{~s}^{-1}\right)\end{array}$ & $\begin{array}{l}k_{p, R a} \\
\left(\mathrm{~s}^{-1}\right)\end{array}$ & $\begin{array}{l}R_{f, R R}{ }^{*} \\
0\end{array}$ \\
\hline${ }^{226} \mathrm{Ra}$ & & & 1.3 & & & 2485 \\
\hline${ }^{228} \mathrm{Ra}$ & & & 1.1 & & & 10 \\
\hline${ }^{223} \mathrm{Ra}$ & $6.0 \times 10^{-10}$ & $2.2 \times 10^{-9}$ & 1.0 & $2.3 \times 10^{-8}$ & $3.4 \times 10^{-8}$ & 1.0 \\
\hline${ }^{224} \mathrm{Ra}$ & & & 1.0 & & & 1.0 \\
\hline
\end{tabular}

These results are in line with the statement of Rama and Moore (1984) who pointed out that migration through microfractures may restrict the input of short-lived radionuclides because the rate of diffusion through microfractures is so slow that it reduces the effect of alpha recoil supply. However, their study focused on the very short-lived ${ }^{220} \mathrm{Rn}$, and thus, one may have some doubts if it is applicable to isotopes with longer half-lives.

Hammond et al. (1988), for example, suggest that radium reaches the large fractures within a few hours and thus, diffusional flux does not limit the activity of the short-lived $\mathrm{Ra}$ isotopes. Their study focused on the uranium and thorium series radionuclides in brines and reservoir rocks from two deep geothermal boreholes in the Salton Sea Geothermal Field (SSGF), California. From their 
modeling results, they postulated that the observed fluid activity of ${ }^{223} \mathrm{Ra}$ and ${ }^{224} \mathrm{Ra}$ can be explained by alpha recoil, while only half of the ${ }^{228} \mathrm{Ra}$ and even less than $1 \%$ of the ${ }^{226} \mathrm{Ra}$ activity can be explained by alpha recoil mechanisms. In consequence, the residual proportions of the ${ }^{228} \mathrm{Ra}$ and

${ }^{226} \mathrm{Ra}$ activities are contributed by weathering and leaching processes of radium from solid phases occurring on timescales comparable to the half-lives of ${ }^{228} \mathrm{Ra}$ and ${ }^{226} \mathrm{Ra}$. Their approach is supported by an observed deficiency of ${ }^{226} \mathrm{Ra}$ in the SSFG reservoir section (Zukin at al., 1987).

However, in this study the situation is exactly opposite since the previous work of the authors figured out that ${ }^{226} \mathrm{Ra}$ is accumulated in the solids of the Bruchsal reservoir section suggesting that ${ }^{226} \mathrm{Ra}$ is rather removed from brine than leached from solids (Kölbel et al., 2020).

\subsubsection{Ra removal by adsorption and precipitation}

${ }^{226} \mathrm{Ra}$ can be removed from brine either by adsorption or by solid solution formation or both (Langmuir and Melchior, 1985). Equal activities of ${ }^{226} \mathrm{Ra}$ and of its (unreactive) daughter ${ }^{222} \mathrm{Rn}$ indicates that ${ }^{226} \mathrm{Ra}$ is rarely adsorbed. Referring to Eqn. (4.15) retardation factor is not only a function of $k_{1}$ and $k_{2}$, but is also dependent on the decay constant of the respective nuclide. Should the desorption rate constant $k_{2}$ be much greater than the decay constant of ${ }^{224} \mathrm{Ra}\left(\lambda_{224 \mathrm{R} a}=2.209 \times 10^{-6} \mathrm{~s}^{-1}\right)$ then $R_{f, 224 R a}=R_{f, 223 \mathrm{R} a}=R_{f, 228 R a}=R_{f, 226 \mathrm{Ra}}$ (Luo et al., 2000) applies. Otherwise short-lived radium isotopes will undergo less retardation than the long-lived ${ }^{226} \mathrm{R}$ a due to their widely ranging half-lives.

Retardation factors for the short-lived radium isotopes might be calculated from the approach suggested by Krishnaswami (Eqn. (4.22ff)). However, deviations in the $P / \lambda C$ ratio from unity might rather result from ${ }^{224} \mathrm{R}$ a depletion in large fractures than from adsorption.

Therefore, only some general consideration about the retardation of radium could be made. Assuming that radium behaves mostly conservative $\left({ }^{226} \mathrm{Ra} /{ }^{222} \mathrm{Rn}=1.3\right)$ and that the desorption rate constant is small compared to the ${ }^{224} \mathrm{Ra}$ decay constant which is likely because of the high radium solubility in the geothermal brine, short-lived ${ }^{224} \mathrm{Ra}$ should experience less retardation than ${ }^{226} \mathrm{Ra}$ $\left(R_{f, 224 \mathrm{R} a} \neq \mathrm{R}_{f, 226 \mathrm{R} a}\right)$. Hence, considering a min/max approach $\left(R_{f, \text { min }}=R_{f, 224 \mathrm{R} a}=1.0 ; R_{f, \text { max }}=R_{f, 226 \mathrm{R} a}=\right.$ 1.3) with respect to the isotope half-lives, adsorption-desorption rates constants are in order of 10${ }^{10} \mathrm{~s}^{-1}$ for $k_{1}$ and $10^{-9} \mathrm{~s}^{-1}$ for $k_{2}$ (Table 4.12).

Previous studies of the adsorption behavior of radium in high-temperature and high-saline natural waters indicate that scavenging of radium by sorption processes might be of minor importance. Tanner (1964) proposed that during cation exchange Ra adsorption may be reduced because of the competition between radium and other alkaline earth metals for sorption sites resulting in an enrichment of radium in saline waters due to Ra-displacement from the rock surface by other cations with higher affinity to the exchange sites. 
A rough estimate of the quantity of radium adsorbed on the rock surface may be calculated employing MIN3P. For calculations, an average cation exchange capacity (CEC) value of 2.00 meq / $100 \mathrm{~g}$ was chosen since the reservoir material in Bruchsal mainly consists of quartz-dominated sandstones $\left(\mathrm{CEC}_{\text {quartz }}=0.6 \mathrm{meq} / 100 \mathrm{~g}\right.$ according to Carroll, 1959).

Thermodynamic data for cation exchange were taken from PHREEQC2-database (Parkhurst and Appelo, 1999). With respect to the chemical composition of the Bruchsal brine (Table 4.1), radium competes with $\mathrm{Na}, \mathrm{K}, \mathrm{Mg}, \mathrm{Sr}, \mathrm{Ba}$ and $\mathrm{Cs}$ for cation exchange sites. The results show that the adsorbed radium species vary between $10^{-17}$ and $10^{-12} \mathrm{meq} / 100 \mathrm{~g}$ corresponding to radium distribution coefficients $K_{d}$ of 0.014 to $0.016 \mathrm{~mL} \mathrm{~g}^{-1}$.

However, these model derived $K_{d}$ values may differ from the in-situ distribution coefficients. Thus, the control of radium activities by adsorption cannot yet be proven without further site-specific investigations regarding the chemical behavior of radium for different environmental conditions.

Should adsorption play a minor role, ${ }^{226} \mathrm{Ra}$ activity should be controlled by co-precipitation. Hammond et al. (1988) pointed out that the determination of first-order precipitation rate $k_{p}\left(\mathrm{~s}^{-1}\right)$ and dissolution rate constants $k_{w}\left(\mathrm{~s}^{-1}\right)$, respectively, may be obtained from solving simultaneously mass balance equations for radium isotopes. Combining Eqn. (4.16) with Eqn. (4.18), (4.19), (4.25) and (4.26) and assuming $C^{i}=0$, the mass balance equation for radium at steady state can be expressed as:

$$
\overline{A^{\prime}} e_{i} \varepsilon_{222} r S \rho_{s}+\frac{k_{w} \bar{A}}{\lambda}-\left(1+\frac{k_{p}}{\lambda}+\frac{1}{\lambda \tau_{b}}\right) A=0
$$

Simultaneous solution of Eqn. (4.40) was performed for ${ }^{226} \mathrm{Ra}$ and ${ }^{228} \mathrm{Ra}$, the two radium isotopes with half-lives in the order of years. The resulting rate constants are in the range of $10^{-8} \mathrm{~s}^{-1}$ for both, dissolution and precipitation, whereas $k_{p}$ slightly exceeds $k_{w}$ (Table 4.12).

From the calculated rate constants further information can be obtained about radium kinetics. Hammond et al. (1988) argued that the mean time for radium in solution equals the mass of radium in the solid phase divided by the flux into solution expressed as follows

$$
\tau_{w, R a}=\frac{M_{r}}{M_{b}} \frac{\bar{A}}{\lambda C} \frac{1}{k_{w}}
$$

where $M_{b}$ is the mass of brine and $M_{r}$ is the mass of rock. Assuming a fracture porosity of $1 \%$ (since the ${ }^{226} \mathrm{Ra}$ accumulation in the reservoir rock was limited to fractured zones), the mean time for radium dissolution is predicted to be approximately 500 years. The mean time of radium in solution to precipitate in minerals $\left(\tau_{p, R a}=1 / k_{p}\right)$ is estimated at circa one year. 
The model derived rates for dissolution $\left(P_{w}\right)$ and precipitation $\left(S_{p}\right)$ are listed in Table 4.12 demonstrating that radium will be preferred co-precipitate with minerals than leached from the solid phases.

Langmuir and Melchior (1985) found that the concentrations of dissolved radium in some deep brines in north Texas were likely to be controlled by co-precipitation in sulphate minerals due to the high concentrations of sulphate and earth alkali ions. Barite is a typical sulphate mineral incorporating radium in solid solution as $[\mathrm{Ba}, \mathrm{Ra}] \mathrm{SO}_{4}$. Both earth-alkali ions consist of an equal ionic charge and show similar ionic radii (radium $=1.52 \mathrm{~A}^{\circ}$, barium $=1.35 \mathrm{~A}^{\circ}$ according to Shannon, 1976). The observation by Langmuir and Melchior (1985) is confirmed by current studies dealing with the formation of Ra-bearing barite in German geothermal sites (Heberling at al., 2017; HaasNüesch at al., 2018). From petrographic studies of the Bruchsal reservoir rock it is known that barite often occurs in the reservoir section as a result of hydrothermal activities (Kölbel et al., 2020).

Zhen-Wu et al. (2016) studied barite dissolution and precipitation rates as a function of temperature and aqueous fluid composition. Their results demonstrate that barite readily achieves equilibrium with its adjacent fluid phase over a range of ionic strengths (aqueous $\mathrm{NaCl}$ concentrations $=0$ to 1.5 molal) and in the presence of divalent metal cations ( $\mathrm{Ca}, \mathrm{Mg}$ and $\mathrm{Sr}$ ) at temperatures ranging from 25 to $90{ }^{\circ} \mathrm{C}$. They concluded that aqueous solution-barite equilibrium is broadly achieved in nature.

Although reservoir temperature and molality of the $\mathrm{NaCl}$ geothermal brine are slightly increased compared to the experimental conditions $\left(\mathrm{T}=134.7^{\circ} \mathrm{C} ; \mathrm{M}(\mathrm{NaCl})=2.1 \mathrm{~mol} \mathrm{~kg}{ }^{-1}\right)$, rate constants for $k_{p}$ and $k_{w}$ presented by Zhen-Wu et al. (2016) are in the same order of magnitude as those derived by the mass balance approach. Consequently, Ra removal from brine by co-precipitation with barite might be a possible explanation for the ${ }^{226} \mathrm{Ra}$ anomaly observed in the Bruchsal geothermal reservoir.

\subsubsection{Ra supply by groundwater flow}

Assuming a water recharge in the Black Forest at the Eastern main border of the URG as suggested by several numerical models of coupled heat- and fluid-flow (e.g. Clauser and Villinger, 1990), meteoric water infiltrates into the Permo-Triassic aquifer of the Rhine Graben. Since this infiltrating water is low in natural occurring radionuclides, the initial concentration of radium can be assumed to be negligible $\left(\mathrm{C}^{\mathrm{i}}=0\right.$ atoms $\left.\mathrm{kg}^{-1}\right)$.

The water transit time $\tau_{b}$ is estimated at 5000 years (assuming a flow distance of $x=5 \mathrm{~km}$ and a fluid velocity $\left.v_{f}=1 \mathrm{~m} \mathrm{yr}^{-1}\right)$. Calculation of $Q$ by applying Eqn. (4.18) results in negative values which indicate radium loss rather than radium supply due to mass transport in groundwater. However, $Q$ is only notable for ${ }^{226} \mathrm{Ra}$ (cf. Table 4.13), since the rate of radium loss for ${ }^{228} \mathrm{Ra},{ }^{223} \mathrm{Ra}$ and ${ }^{224} \mathrm{Ra}$ is $\leq$ $2.6 \times 10^{-2}$ atoms $\mathrm{kg}^{-1} \mathrm{~s}^{-1}$. 


\subsubsection{Steady state Ra fluid activities}

At steady state, the removal rates (activity of dissolved and adsorbed radium and precipitation) are equal to inputs (radium recoil rate and production from dissolved and absorbed Th progenitors), so that (Porcelli et al., 2008):

$$
A_{R a, \text { steady }}=\frac{P_{r}+P_{w}}{R_{f}+\left(\frac{k_{p}}{\lambda}\right)}=\frac{P_{r}+P_{w}}{R_{f}^{*}}
$$

Table 4.13: Model derived rates production and scavenging rates (atoms $\left.\mathrm{kg}^{-1} \mathrm{~s}^{-1}\right)$ for radium in the Bruchsal geothermal system.

\begin{tabular}{|c|c|c|c|c|c|}
\hline Water-rock interaction processes & & ${ }^{226} \mathrm{Ra}$ & ${ }^{228} \mathrm{Ra}$ & ${ }^{223} \mathrm{Ra}$ & ${ }^{224} \mathrm{Ra}$ \\
\hline $\begin{array}{l}\text { Supply from alpha recoil in consideration of the } \\
\text { diffusional flux }\end{array}$ & $P_{r}^{*}$ & 244 & 56 & 0.7 & 10 \\
\hline Production from desorption & $P_{d}$ & 1,268 & 1 & 0.0 & 0.0 \\
\hline Production from dissolution & $P_{w}$ & 72,391 & 102 & 0.0 & 0.0 \\
\hline Scavenging from adsorption & $S_{a}$ & 1,276 & 2 & 0.0 & 0.0 \\
\hline Scavenging from precipitation & $S_{p}$ & 72,585 & 141 & 0.0 & 0.0 \\
\hline Supply by water flow & $Q$ & -13 & 0.0 & 0.0 & 0.0 \\
\hline Steady state activity & $A_{\mathrm{R} a}$ & 29 & 16 & 0.7 & 10 \\
\hline
\end{tabular}

Eqn. (4.42) might be used as a control for the discussed water-rock interactions since it merges the single interaction processes which should lead to the radium fluid activities measured in the Bruchsal brine. The denominator of the fraction reflects the retardation factor $R_{f}^{*}$ due to precipitation as well as adsorption and desorption. Since $R_{f}^{*}$ does not differ from $R_{f}$ for short-lived radionuclides, $\mathrm{R}_{f}^{*}$ will be increase with decreasing decay constants (Table 4.12).

Table 4.13 summarizes radium production and removal rates of the water-rock interaction processes discussed. From the results it becomes obvious that for ${ }^{224} \mathrm{Ra}$ and ${ }^{223} \mathrm{Ra}$, precipitation and dissolution processes can be neglected and so removal by decay is equal to inputs from recoil for ${ }^{228} \mathrm{Th}$ and ${ }^{227} \mathrm{Th}$, respectively, within the solids for steady state conditions. On the other hand, it is interesting to note that not only ${ }^{226} \mathrm{Ra}$, but also ${ }^{228} \mathrm{Ra}$ is affected by dissolution-precipitation processes. This is in line with the statement of Hammond et al. (1988) who postulated that these processes occur on timescales comparable to the half-lives of ${ }^{228} \mathrm{Ra}$ and ${ }^{226} \mathrm{Ra}$.

Model derived Ra steady state activities are in good agreement with the observed Ra fluid activities (cf. Table 4.5) which support the applicability of the diffusional flux model. The diffusion of radium 
through microfractures do not only restrict the short-lived radionuclides as it is postulated by Rama and Moore (1984), but it has also a reinforcing effect on the ${ }^{226} \mathrm{Ra}$ activity due to its relatively high diffusional flux.

\subsection{Conclusions}

In this study we investigated the behavior of U-Th series radionuclides in the brine of the Bruchsal geothermal site, located at the eastern main boundary of the Upper Rhine Graben (Germany). Permo-Triassic sedimentary rocks, affected by large-scale normal faults, host the geothermal reservoir.

Isotopes of $\mathrm{Ra}\left({ }^{226} \mathrm{Ra},{ }^{228} \mathrm{Ra},{ }^{224} \mathrm{Ra},{ }^{223} \mathrm{Ra}\right), \mathrm{Rn}\left({ }^{222} \mathrm{Rn}\right)$ and $\mathrm{Pb}\left({ }^{210} \mathrm{~Pb},{ }^{212} \mathrm{~Pb}\right), \mathrm{U}\left({ }^{238} \mathrm{U},{ }^{234} \mathrm{U},{ }^{235} \mathrm{U}\right)$, Th $\left({ }^{232} \mathrm{Th},{ }^{228} \mathrm{Th},{ }^{230} \mathrm{Th}\right)$, Po $\left({ }^{210} \mathrm{Po}\right)$ and $\mathrm{Ac}\left({ }^{227} \mathrm{Ac},{ }^{228} \mathrm{Ac}\right)$ were analyzed over a sampling period from October 2016 to May 2017.The results show discrepancies between the fluid activities of the Th$\mathrm{U}$ series nuclides measured in the Bruchsal brine: while isotopes of $\mathrm{U}, \mathrm{Th}, \mathrm{Ac}$ and Po are below the limit of analytical determination $\left(<10^{-2} \mathrm{~Bq} \mathrm{~kg}^{-1}\right)$, isotopes of $\mathrm{Rn}\left({ }^{222} \mathrm{Rn}=38 \mathrm{~Bq} \mathrm{~kg}{ }^{-1}\right), \mathrm{Ra}\left({ }^{226} \mathrm{Ra}\right.$ $\left.=29 \mathrm{~Bq} \mathrm{~kg}^{-1} ;{ }^{228} \mathrm{Ra}=16 \mathrm{~Bq} \mathrm{~kg}^{-1 ; 224} \mathrm{Ra}=11 \mathrm{~Bq} \mathrm{~kg}^{-1 ; 223} \mathrm{Ra}=0.5 \mathrm{~Bq} \mathrm{~kg}^{-1}\right)$ and $\mathrm{Pb}\left({ }^{210} \mathrm{~Pb}=26 \mathrm{~Bq} \mathrm{~kg}^{-1}\right.$; ${ }^{212} \mathrm{~Pb}=16 \mathrm{~Bq} \mathrm{~kg}^{-1}$ ) are rather soluble.

Differences in chemical and physical properties result in radioactive disequilibria. Modeling the disequilibria based on radium $\left({ }^{226} \mathrm{Ra},{ }^{228} \mathrm{Ra},{ }^{224} \mathrm{Ra},{ }^{223} \mathrm{Ra}\right)$ enabled us to estimate rate constants of water-rock interactions. Since the daughter-parent ratio of ${ }^{222} \mathrm{Rn} /{ }^{226} \mathrm{Ra}$ is ca. 1.3 , Ra retardation due to sorption processes is small resulting in adsorption-desorption rate constants in the range of $10^{-}$ ${ }^{10} \mathrm{~s}^{-1}$ for $k_{1}$ and $10^{-9} \mathrm{~s}^{-1}$ for $k_{2}$. Model derived Ra distribution coefficients $K_{d}$ vary between $0.014-$ $0.016 \mathrm{~mL} \mathrm{~g}^{-1}$. First-order precipitation rate constant $\left(k_{p}=3.4 \times 10^{-8} \mathrm{~s}^{-1}\right)$ slightly exceeds those of dissolution $\left(k_{w}=1.2 \times 10^{-8} \mathrm{~s}^{-1}\right)$. Precipitation occurs on timescales comparable to ${ }^{226} \mathrm{Ra}$ and ${ }^{228} \mathrm{Ra}$, while the short-lived ${ }^{224} \mathrm{Ra}$ and ${ }^{223} \mathrm{Ra}\left(\mathrm{t}_{1 / 2}=3.66\right.$ and 11.43 days, respectively) are not affected. Indeed, the short-lived $\mathrm{Ra}$ isotopes are mostly supplied from alpha recoil.

Assuming a fracture porosity of $1 \%$, the average time to leach all ${ }^{226} \mathrm{Ra}\left(=M_{r} A_{r} \lambda^{-1}\right)$ from solid phases is predicted at ca. 500 years, while the average time of dissolved radium to co-precipitate in minerals is estimated at circa one year.

Since fractured zones provide a substantial portion of the permeability due to the low porosity of the Bruchsal sandstone reservoir rock, the observed decay-series disequilibria in brine can best be explained by the following conclusions on water-rock interaction processes:

(1) The hydraulically inactive pore spaces and microfractures, respectively, are the main source for alpha recoil, while recoil supply from large fractures may be negligible due to their relative low brine-rock interaction rates. 
(2) Diffusional flux through pore spaces and microfractures is believed to supply radionuclides to the large conductive fractures causing a significant fractionation of Ra isotopes.

(3) Migration through microfractures may limit input of short-lived Ra isotopes into solution, depending on isotope half-lives and fracture geometry.

(4) Radium removal is rather by co-precipitation with solid solutions than by sorption processes which is indicated by the isotopic ratio of ${ }^{222} \mathrm{Rn} /{ }^{226} \mathrm{Ra}=1.3$ in brine.

(5) Co-precipitation of radium is most likely for barite and restricted to the fractured reservoir section.

From (1) and (2) it can be concluded that the recoil input is strongly diffusion-controlled which might be used to gain information about the fracture surface area (Andrews et al., 1989). For the short-lived ${ }^{223} \mathrm{Ra}$ and ${ }^{224} \mathrm{Ra}$, alpha recoil is the most important process to enter the fluid system. Thus, the activity of ${ }^{223} \mathrm{Ra}\left({ }^{224} \mathrm{Ra}\right)$ dissolved within the large fracture fluid depends on the diffusional flux from rock surfaces and the size of the fracture. Estimates about the specific surface area $\left(\mathrm{m}^{2}\right.$ $\left.\mathrm{m}^{-3}\right)$ may be deduced from the known flux $\left(F_{R a}\right)$ and the fluid activity $\left(A_{R a}\right)$ of the radium isotopes. In this case, the specific surface area is defined as the surface area of solids per volume of fracture fluid contacting the solids (which corresponds to the flow wetted surface area). Since the specific surface area of the fracture is inversely proportional to the fracture width, further information about the site-specific fracture geometry can be obtained. However, it should be noted that this fracture width represents an averaged equivalent aperture since the fluid samples analyzed are mostly collected from the larger aperture, hydraulically conductive fractures. Thus, the fracture surface area depends on (1) whether the estimated fracture width represents one large or several smaller fractures and (2) the extent of the variance from the averaged equivalent fracture width. With regard to reservoir engineering, it might be interesting to solve this question more detailed. Based on the determination of the averaged equivalent aperture, additional numerical modelling of heat transfer is a promising option to solve these questions.

\section{Acknowledgements}

This research was carried out within the framework of the ANEMONA project, funded by the German Federal Ministry for Economic Affairs and Energy (BMWi). We are also grateful to the laboratory of IAF (Dresden) that performed the alpha-ray measurements and the laboratory of EnBW Kernkraft GmbH where the gamma spectrometry surveys were carried out. We especially thank G. Gkogkidis for his great support in setting up the gamma spectrometric measuring stations. 


\section{References}

Allison, J. D., Brown, D. S., Novo-Gradac, K. J., 1991. MINTEQA2/PRODEFA2; A geochemical assessment model for environmental systems, version 3.0, users’s manual, EPA/600/3-91/021, Environ. Res. Lab., U.S.

Environ Prot. Agency, Washington, D. C.

Andrews, J. N., Giles, I. S., Kay, R. L. F., Lee, D. J., Osmond, J. K., Cowart, J. B., Fritz, P., Barker, J. F., Gale, J., 1982. Radioelements, radiogenic helium and age relationships for groundwaters from the granites at Stripa, Sweden. Geochimica et Cosmochimica Acta, 46, 1533-1543.

Andrews, J. N., Ford, D. J., Hussain, N., Trevedi, D., Youngman, M. J., 1989. Natural radioelement solution by circulating groundwaters in the Stripa granite. Geochimica et Cosmochimica Acta, 53, 1791-1802.

Attendorn, H.G., Bowen, R.N.C., 1997. Radioactive and Stable Isotope Geology. Chapman and Hall, London, UK, p. 522.

Bateman, H. (1910). Solution of a system of differential equations occurring in the theory of radioactive transformations. Proceedings of the Cambridge Philosophical Society, 15, 423-427.

Beaucaire C, Criaud A, Michard G, 1987. Contrôle des concentrations de certains éléments traces (As, Sb, U, Ra, Ba) dans les eaux du Cézallier (Massif Central, France). Chemical Geology 63, 85-99.

Carroll, D., 1959. Ion exchange in clays and other minerals. Geological Society of America Bulletin 70, 749-780.

Clauser, C., Villinger, H., 1990. Analysis of conductive and convective heat transfer in a sedimentary basin, demonstration for the Rheingraben, Geophys. J. Int. 100, 393-414.

Condomines, M., Rihs, S., Lloret, E., Seidel, J.L., 2010. Determination of the four natural Ra isotopes in thermal waters by gamma-ray spectrometry. Appl. Radiat. Isot. 68, 384-391.

Condomines, M., Gourdin, E., Gataniou, D., Seidel, J.-L., 2012. Geochemical behaviour of Radium isotopes and Radon in a coastal thermal system (Balaruc-les-Bains, South of France). Geochimica et Cosmochimica Acta 98, 160-176.

Davidson, M. R., Dickson, B. L., 1986. A porous flow model for steady-state transport of radium in ground waters. Water Resources Research, 22, 34-44.

Degering, D., Köhler, M., 2011. Gamma-spectrometric analysis of high salinity fluids - how to analyze radionuclides of the thorium decay chain far from radioactive equilibrium? Applied Radiation and Isotopes 69, 1613-1617.

Dickson, B.L., 1985. Radium isotopes in saline seepage, southwestern Yilgarn, Western Australia. Geochim. Cosmochim. Acta 49, 349-360.

Eggeling, L., Genter, A., Kölbel, T., Münch, W., 2013. Impact of natural radionuclides on geothermal exploitation in the Upper Rhine Graben. Geothermics 47, 80-88. 
Foster D A, Staubwasser M, Henderson G M, 2004. ${ }^{226} \mathrm{Ra}$ and Ba concentrations in the Ross Sea measured with multicollector ICP mass spectrometry. Marine Chemistry, 87, 59- 71.

Gascoyne, M. (1992). Geochemistry of the actinides and their daughters. In: Uranium-Series Disequilibrium Applications to Earth, Marine, and Environmental Sciences (Eds M. Ivanovich and R. S. Harmon). Clarendon Press, Oxford, pp. 34-61.

Grandia, F., Merino, J., Bruno, J., 2008. Assessment of the radium-barium co-precipitation and its potential influence on the solubility of Ra in the near-field Stockholm, Swedish Nuclear Fuel and Waste Management Co: 52.

Grundl T, Cape M, 2006. Geochemical factors controlling radium activity in a sandstone aquifer. Ground Water, 444, 518-527.

Haas-Nüesch, R., Heberling, F., Schild, D., Rothe, J., Dardenne, K., Jähnichen, S., Eiche, E., Marquardt, C., Metz, V., Schäfer, T., 2018: Mineralogical characterization of scalings formed in geothermal sites in the Upper Rhine Graben before and after the application of sulfate inhibitors. In: Geothermics 71, 264-273.

Hammond, D.E., Zukin, J.G., Ku, T.L., 1988. The kinetics of radioisotope exchange between brine and rock in a geothermal system. J. Geophys. Res. 93 (13), 175-186.

Heap, M.J., Villeneuve, M., Kushnir, A.R.L., Farquharson, J.I., Baud, P., Reuschlé, T., 2019. Rock mass strength and elastic modulus of the Buntsandstein: An important lithostratigraphic unit for geothermal exploitation in the Upper Rhine Graben. Geothermics 77, 236-256.

Heberling, F., Schild, D., Degering, D., Schäfer, T., 2017. How well suited are current thermodynamic models to predict or interpret the composition of $(\mathrm{Ba}, \mathrm{Sr}) \mathrm{SO} 4$ solid-solutions in geothermal scalings? In: Geotherm Energy 5 (1).

Jerez-Vegueria S F, Godoy J M, Miekeley N, 2002. Environmental impact studies of barium and radium discharges by produced waters from the "Bacia de Campos" oil-field offshore platforms, Brazil. Journal of Environmental Radioactivity 62, 29-38.

Joachim, H., Koziorowski, G., Leiber, J., 1987. Geothermiebohrungen Bruchsal 1a und 2, in: Maus, H. (Ed.), Jahreshefte des Geologischen Landesamtes Baden-Württemberg 29. Herder, Freiburg i. Br., pp. 84-97.

Kigoshi, K. (1971). Alpha recoil ${ }^{234} \mathrm{Th}$ : Dissolution in water and the ${ }^{234} \mathrm{U} /{ }^{238} \mathrm{U}$ disequilibrium in nature. Science, 173 , 47-48.

Kramer, T.F., Reid, D.F., 1984. The occurrence and behavior of radium in saline formation water of the U.S. Gulf Coast region. Isotope Geoscience 2, 153- 174.

Krishnaswami, S., Graustein, W.C., Turekian, K.K., Dowd, J.F., 1982. Radium, thorium and radioactive lead isotopes in groundwaters: Applications to the in situ determination of adsorption-desorption rate constants and retardation factors. Water Resour. Res. 18 (6), 1633-1675.

Kölbel, L., Kölbel, T., Wiegand, B., Sauter, M., Schäfer, T., Siefert, D., 2020. Identification of fractured zones in 
geothermal reservoirs in sedimentary basins: A radionuclide-based approach. Geothermics 85, 101764.

Ku, T.-L., Luo, S., Leslie, B.W., Hammond, D.E., 1992. Decay-series disequilibria applied to the study of water-rock interaction and geothermal systems, in: Ivanovich, M., Harmon, R.S. (Eds.), Uranium-series Disequilibrium: Applications to Earth, Marine, and Environmental Sciences. Clarendon Press, Oxford, 631-668.

Ku, T. L., Luo, S., Leslie, B.W., Hammond, D.E., 1998. Assessing in-situ radionuclide migration from natural analog studies: Response to McKinley and Alexander (1996). Radiochimica Acta 80, 219-223.

Langmuir, D., Melchior, D., 1985. The geochemistry of Ca, Sr, Ba, and Ra sulfates in some deep brines from the Palo Duro Basin, Texas. Geochim. Cosmochim. Acta 49, 2423-2432.

Lasaga, A.C., 1998. Kinetic Theory in the Earth Sciences. Princeton Univ. Press, Princeton, NJ.

Luo, S., Ku, T.-L., Roback, R., Murrell, M., McLing, T.L., 2000. In-situ radionuclide transport and preferential groundwater flows at INEEL (Idaho): Decay-series disequilibrium studies. Geochim. Cosmochim. Acta, 64, 867881.

Mao, S., Duan, Z., 2008. The P, V, T,x properties of binary aqueous chloride solutions up to T = $573 \mathrm{~K}$ and $100 \mathrm{MPa}$. J. Chem. Thermodynamics 40, 1046-1063.

Mayer, K.U., Frind, E.O., Blowes, D.W., 2002. Multicomponent reactive transport modeling in variably saturated porous media using a generalized formulation for kinetically controlled reactions. Water Resour. Res. 38, 1174.

Moise T, Starinsky A, Katz A, Kolodny Y, 2000. Ra isotopes and Rn in brines and ground waters of the Jordan-Dead Sea Rift Valley: Enrichment, retardation, and mixing. Geochimica et Cosmochimica Acta, 64-14, 23712388.

Moore W S, 1997. High fluxes of radium and barium from the mouth of the Ganges Brahmaputra River during low river discharge suggest a large groundwater source. Earth and Planetary Science Letters, 150 141-150.

Nozaki Y, Yamamoto Y, Manaka T, Amakawa H, Snidvongs A, 2001. Dissolved barium and radium isotopes in the Chao Phraya River estuarine mixing zone in Thailand. Continental Shelf Research 21, 1435-1448.

Osmond, J.K., Cowart, J.B., 1992. Ground water. In: Ivanovich, M., Harmon, R.S. (Eds.), Uranium-series Disequilibrium: Applications to Earth, Marine, and Environmental Sciences. Oxford Science Publications, pp. 290.

Parkhurst, D.L. and Appelo, C.A.J., 1999. User's guide to PHREEQC - A computer program for speciation, reactionpath, 1D-transport, and inverse geochemical calculations: Technical Report 99 - 4259, US Geol. Survey Water-Resources Investigations Report.

Parkhurst, D.L., Appelo, C.A.J., 2013. Description of Input and Examples for PHREEQC Version 3-A Computer Program for Speciation, Batch-Reaction, One-Dimensional Transport, and Inverse Geochemical Calculations. U.S. Department of the Interior, U.S. Geological Survey.

Porcelli, D., 2008. Investigating groundwater processes using U- and Th-series nuclides, in: Baxter, M.S. (Ed.), Radioactivity in the Environment 13. Elsevier, Amsterdam, 105-153. 
Porcelli, D., Kim, C.-K., Martin, P., Moore, W.S., Phaneuf, M., 2014. Properties of radium, in: IAEA (Ed.), the environmental behavior of radium (revised edition), technical report series no. 476, Vienna, 6-32.

Pribnow, D., Schellschmidt, R., 2000. Thermal tracking of upper crustal fluid flow in the Rhine Graben. Geophysical Research Letters 27, 1957-1960.

Rama and Moore, W.S., 1984. Mechanism of transport of U-Th series radioisotopes from solids into ground water. Geochim. Cosmochim. Acta 48, 395-400.

Rihs S, Condomines M, Sigmarsson O, 2000. U, Ra and Ba incorporation during precipitation of hydrothermal carbonates: Implications for 226Ra-Ba dating of impure travertines. Geochimica et Cosmochimica Acta 64, 661671.

Rihs S. and Condomines M., 2002. An improved method for Ra isotope $\left({ }^{226} \mathrm{Ra},{ }^{228} \mathrm{Ra},{ }^{224} \mathrm{Ra}\right)$ measurements by gamma spectrometry in natural waters: applications to $\mathrm{CO}_{2}$-rich thermal waters from the French Massif Central. Chem. Geol. 182, 409-421.

Rodriguez-Galan, R. M., Prieto, M., 2018. Interaction of nonideal, multicomponent solid solutions with water: A simple algorithm to estimate final equilibrium states. Geochemistry, Geophysics, Geosystems, 19, 1348-1359.

Shannon, R.D., 1976. Revised effective ionic radii and systematic studies of interatomic distances in halides and chalcogenides. Acta Crystallographica 32, 751-767.

Sturchio N, Bohlke J, Markun F, 1993. Radium geochemistry of geothermal waters, Yellowstone National Park, Wyoming, USA. Geochimica et Cosmochimica Acta 57, 1203-1214.

Sturchio N, Banner J, Binz C, Heraty L, Musgrove M, 2001. Radium geochemistry of ground waters in Paleozoic carbonate aquifers, mid-continent, USA. Applied Geochemistry 16, 109-122.

Sun, H., Semkow, T., 1998. Mobilization of thorium, radium and radon radionuclides in ground water by successive alpha-recoil. Journal of Hydrology 205, 126-136.

Tanner, A.B.,1964. Radon migration in the ground: a review. In: Adams, J.A.S. and Lowder, W.M. (eds), The Natural Radiation Environment, Chicago, 161-190.

Tricca, A., D. Porcelli, and G. J. Wasserburg., 2000. Factors controlling the ground water transport of U, Th, Ra, and Rn. Proceedings of the Indian Academy of Sciences, 109, 95-108.

Tricca, A., Wasserburg, G.J., Porcelli, D., Baskaran, M., 2001. The transport of U- and Th-series nuclides in a sandy unconfined aquifer. Geochim. Cosmochim. Acta 65, 1187-1210.

Zhen-Wu., B.Y., Dideriksen, K., Olsson, J., Raahauge, P.J., Stipp, S.L.S., Oelkers, E.H., 2016. Experimental determination of barite dissolution and precipitation rates as a function of temperature and aqueous fluid composition. Geochimica et Cosmochimica Acta 194, 193-210.

Ziegler, P.A., 1992. European Cenozoic rift system. Tectonophysics 208, 91-111. 
Zukin, J.G., Hammond, D.E., Ku, T.-L., Elders, W.A., 1987. Uranium-Thorium series radionuclides in brines and reservoir rocks from two deep geothermal boreholes in the Salton Sea geothermal field, southeastern California. Geochim. Cosmochim. Acta 51, 2719-2731. 


\section{Chapter V}

\section{Integrated approach into the characterization of the fracture network of a geothermal reservoir}

Lena Kölbel ${ }^{\text {a, }}$, Thomas Kölbel ${ }^{\text {b }}$, Martin Sauter ${ }^{\mathrm{a}}$, Bettina Wiegand ${ }^{\mathrm{a}}$, Iulia Ghergut ${ }^{\mathrm{a}}$

Manuscript:

Kölbel, L., Kölbel, T., Sauter, M., Wiegand, B., Ghergut, I., 2020. Integrated approach into the characterization of the fracture network of a geothermal reservoir.

\footnotetext{
${ }^{a}$ University of Göttingen, Geosciences Center, Applied Geology, Goldschmidtstr. 3, 37077 Göttingen, Germany

b EnBW Energie Baden-Württemberg AG, Research and Innovation Department, Durlacher Allee 93, 76131 Karlsruhe, Germany
} 


\section{Abstract}

As an inert gas ${ }^{222} \mathrm{Rn}$ can be employed as a natural radiotracer for the characterization of hydrogeological properties of geothermal systems. In fractured reservoirs, diffusion through non-conductive rock matrices allows recoiled ${ }^{222} \mathrm{Rn}$ to enter larger, hydraulically conductive fractures. In this study, we examine the possibility of assessing fracture dimensions from ${ }^{222} \mathrm{Rn}$ activity in geothermal brines. ${ }^{222} \mathrm{Rn}$ activity in the geothermal fluid is mainly controlled by the ${ }^{222} \mathrm{Rn}$ flux from fracture surfaces and the fracture geometry (aperture, length, degree of connectivity, etc.).

Estimates of site-specific fracture length and width were derived by a ${ }^{222} \mathrm{Rn}$ mass balance approach for the geothermal reservoir at Bruchsal (Germany). Integral hydraulic parameters of the Bruchsal reservoir were obtained from classical hydraulic tests. The results show a ${ }^{222} \mathrm{Rn}$ diffusion flux of 4.2 $\times 10^{-3}$ atoms s$~^{-1} \mathrm{~cm}^{-2}$ resulting in an equivalent fracture width of $10 \mathrm{~mm}$. The equivalent fracture length varies between a few hundred meters as a function of the flow velocity where about $250 \mathrm{~m}$ correspond to a mean flow velocity of $2.0 \times 10^{-6} \mathrm{~m} \mathrm{~s}^{-1}$.

Since the specific surface area of the fracture is inversely proportional to the fracture width, the fracture surface area depends on (1) the type of fracture system, i.e. whether the estimated equivalent fracture width represents a single large fracture or a fracture network of a large number of small aperture fractures and (2) the extent of the variance from the averaged equivalent fracture width. The joint inversion of the radon diffusion model with the pumping test results and a heat transport model is expected to considerably reduce interpretative hydraulic parameter ambiguity 


\subsection{Introduction}

Geothermal reservoirs are largely composed of fractured rocks and the reservoir performance significantly depends on the presence and permeability of fractures (e.g. Grant and Bixley, 2011). The precise evaluation of the effects of fractures on fluid flow is crucial for a successful reservoir management. However, this task is not without difficulties, because fracture geometry is at least in initial project stage unknown and quite complex to assess. Several authors (e.g. Gringarten and Witherspoon, 1972; Raghavan, 1977) suggested a model of a single vertical 'equivalent' fracture that may approximate the hydraulic behavior of the naturally fractured system.

Estimates of the flow regimes are generally based on hydrogeological data such as well test data. We propose that the natural ${ }^{222} \mathrm{Rn}$ flux provides a complementary tool to estimate site-specific fracture dimensions such as fracture width, length, and surface area. In the past, ${ }^{222} \mathrm{Rn}$ has been used extensively as a radiotracer for the study of geological und thermodynamic properties of groundwaters and geothermal systems since it is commonly found in these fluids (e.g. Hammond et al, 1988a, Ku et al., 1992).

${ }^{222} \mathrm{Rn}$ is an intermediate short-lived daughter nuclide $\left(t_{1 / 2}=3.84\right.$ days $)$ of the ${ }^{238} \mathrm{U}$ decay series. It is produced by alpha decay of the ${ }^{226} \mathrm{Ra}$ parent nuclide (Fig. 5.1). Since ${ }^{222} \mathrm{Rn}$ is a noble gas, it is considered to be inert and not reacting with aquifer solids. It is also not affected by time-consuming processes such as precipitation due to its short half-life. Thus, ${ }^{222} \mathrm{Rn}$ is only removed from solution by decay. Radon migrates within the rock mass by diffusion and/or advection through pores, macro-pores, and fractures (e.g. Nazaroff and Nero, 1988; Ferry et al., 2002).

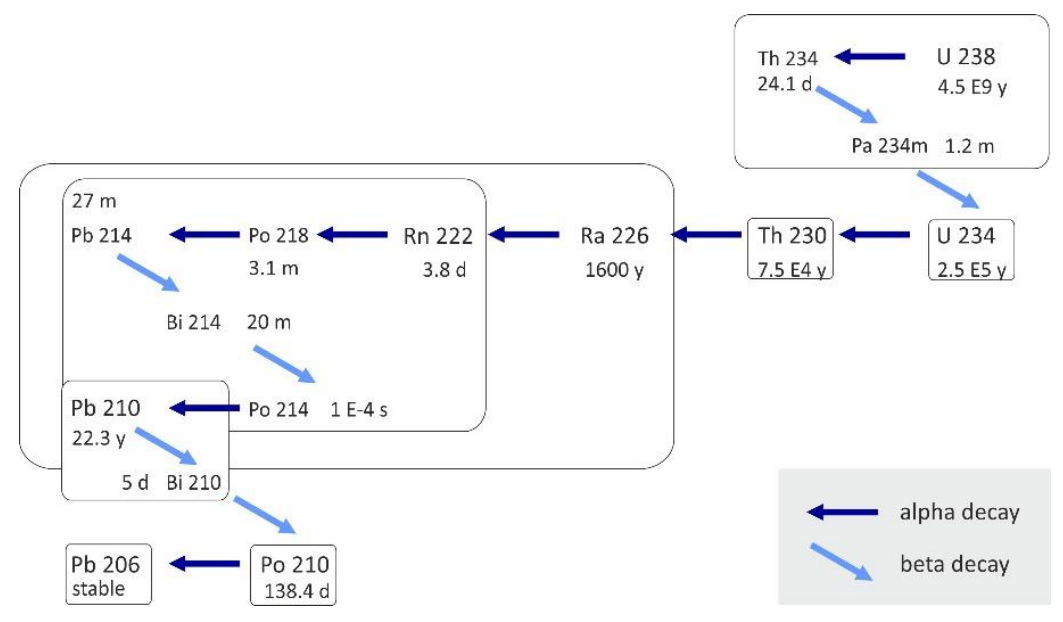

Fig. 5.1: ${ }^{238} \mathrm{U}$ decay series. Vertical arrows indicate alpha decay, diagonal arrows indicate beta decay. For radionuclides with more than one decay mode, only the most frequently occurring is given. 
In low-temperature and low-salinity groundwaters where radium is strongly adsorbed on surfaces, the main supply of ${ }^{222} \mathrm{Rn}$ is from the decay of ${ }^{226} \mathrm{Ra}$ which is within a recoil distance of $\sim 40 \mathrm{~nm}$ of mineral surfaces (Carvalho et al., 2014). However, in high-temperature saline waters such as geothermal brines, radium behaves exactly opposite, resulting in high concentrations of dissolved $\mathrm{Ra}$ isotopes (Hammond et al., 1988; Condomines et al., 2012; Eggeling et al., 2013). Consequently, ${ }^{222} \mathrm{Rn}$ supply is not only from recoil mechanism, but also from the radioactive decay of ${ }^{226} \mathrm{Ra}$ dissolved in brine.

Rama and Moore (1984) suggested that most of the ${ }^{222} \mathrm{Rn}$ is initially introduced via alpha recoil into a network of nanopores and then diffuses along crystal defects, grain boundaries, and hydraulically inactive microfractures into large conductive fractures. Therefore, the ${ }^{222} \mathrm{Rn}$ activity of fluids within large fractures depends on flux from rock surfaces and the size of the fracture. Andrews et al. $(1986,1989)$ investigated ${ }^{222} \mathrm{Rn}$ release in fracture fluids of the Rosemanowes HDR experimental site by direct alpha-recoil, lattice, and grain boundary/microcrack diffusion. They determined the

${ }^{222} \mathrm{Rn}$ flux from plane crystalline rock surfaces into the conductive fracture between two idealized parallel plates to compute fracture apertures, fracture surface areas, and contained volumes.

The present study introduces a mass balance approach of ${ }^{222} \mathrm{Rn}$ applied to the Bruchsal geothermal system to gain information about site-specific fracture geometry. This study is complementary to our previous work where the behavior of ${ }^{222} \mathrm{Rn}$ and other Th-U series radionuclides in Bruchsal brine and the adjacent reservoir rock were extensively studied (Kölbel et al., 2020a, Kölbel et al., 2020b).

\subsection{Geothermal context}

\subsubsection{Upper Rhine Graben}

The Upper Rhine Graben is part of the European Cenozoic Rift System that extends from the Mediterranean to the North Sea coast (Ziegler, 1992). It is characterized by an NNE-SSW striking extension structure with a length of around $300 \mathrm{~km}$ and a width of up to $40 \mathrm{~km}$. Hercynian basement rocks consist of Gneisses and Paleozoic granites which are covered by sediments of Permian to Cenozoic age. The sediment base in the center of the Upper Rhine valley is located about 3000 $\mathrm{m}$ deeper than at the graben shoulders (Ziegler, 1992).

The Upper Rhine Graben offers favorable conditions for the exploitation of geothermal energy because of spatially elevated heat flow and thermal anomalies, which are related to large-scale fluid circulation (Pribnow and Schellschmidt, 2000). 


\subsubsection{Bruchsal site}

The Bruchsal geothermal site is located at the eastern main boundary fault of the Upper Rhine Graben in SW Germany. The geothermal power plant comprises two boreholes: the injection well (GB1) and the production well (GB2) which are located in a distance of $1.5 \mathrm{~km}$ to each other. Caused by the complex tectonic structure, the reservoir section at injection well differs in depth and thickness from the production well.

Permo-Triassic sedimentary rocks, affected by large-scale normal faults with varying step heights between 20 and $350 \mathrm{~m}$, host the geothermal reservoir that is composed of Middle/Lower Buntsandstein (Triassic) to Upper Rotliegend (Permian) rocks in depths of 2220 to $2485 \mathrm{~m}$. The reservoir rock consists of alternating sequences of sandstones, siltstones, and mudstones. Replacement of primary minerals by secondary mineral phases such as the intense transformation of feldspars into clay minerals indicates hydrothermal activities in the reservoir section (Kölbel et al., 2020a).

Fig. 5.2 shows the lithographical profile and the casing/liner scheme of the GB2 production well. The $257 \mathrm{~m}$ long filter section consists of a slotted liner ranging from 2285 to $2542 \mathrm{~m}$ depth. Several geophysical borehole logs were recorded in the 1980's before the liner was installed. Negative temperature gradients, caused by the "cold" drilling fluid along fissure structures, reveal two hydraulically active inflow zones in $2442 \mathrm{~m}$ and $2463 \mathrm{~m}$ depth, both located in the Permian Rotliegend formation (Fig. 5.2). Borehole data of GB2 yielded a bottom hole temperature of $134.7^{\circ} \mathrm{C}$ at 2542 $\mathrm{m}$ depth. This is equal to a geothermal gradient of $55 \mathrm{~K} \mathrm{~km}^{-1}$, which exceeds the average value for Central Europe of $30 \mathrm{~K} \mathrm{~km}^{-1}$.

At present, the hot water is produced with a flow rate of $28 \mathrm{l} / \mathrm{s}$. The Bruchsal brine is highly concentrated in $\mathrm{Cl}, \mathrm{Na}$ and other alkali and alkaline earth elements, containing up to $130 \mathrm{~g} / \mathrm{l}$ total dissolved solids (Joachim et al, 1987). ${ }^{222} \mathrm{Rn}$ and its parent nuclide ${ }^{226} \mathrm{Ra}$ are quite soluble in the geothermal brine with activities up to 38 and 30 atoms s${ }^{-1} \mathrm{~L}^{-1}$, respectively (Table 5.1).

Table 5.1: ${ }^{238} \mathrm{U}$ decay series radionuclides in the Bruchsal brine samples. Relative uncertainties are quoted in percentage $( \pm 2 \sigma$ from counting statistics).

\begin{tabular}{lll}
\hline Radionuclide & Specific activity $\left(\mathrm{Bq} \mathrm{L}^{-1}\right)$ & Relative uncertainty $(\%)$ \\
\hline${ }^{238} \mathrm{U}$ & 0.012 & 59 \\
${ }^{234} \mathrm{U}$ & 0.01 & 66 \\
${ }^{230} \mathrm{Th}$ & $<0.08$ & \\
${ }^{226} \mathrm{Ra}$ & 29.9 & 8.3 \\
${ }^{222} \mathrm{Rn}$ & 38.1 & 7.4 \\
${ }^{210} \mathrm{~Pb}$ & 26.2 & 26 \\
${ }^{210} \mathrm{Po}$ & $<0.1$ & \\
\hline
\end{tabular}




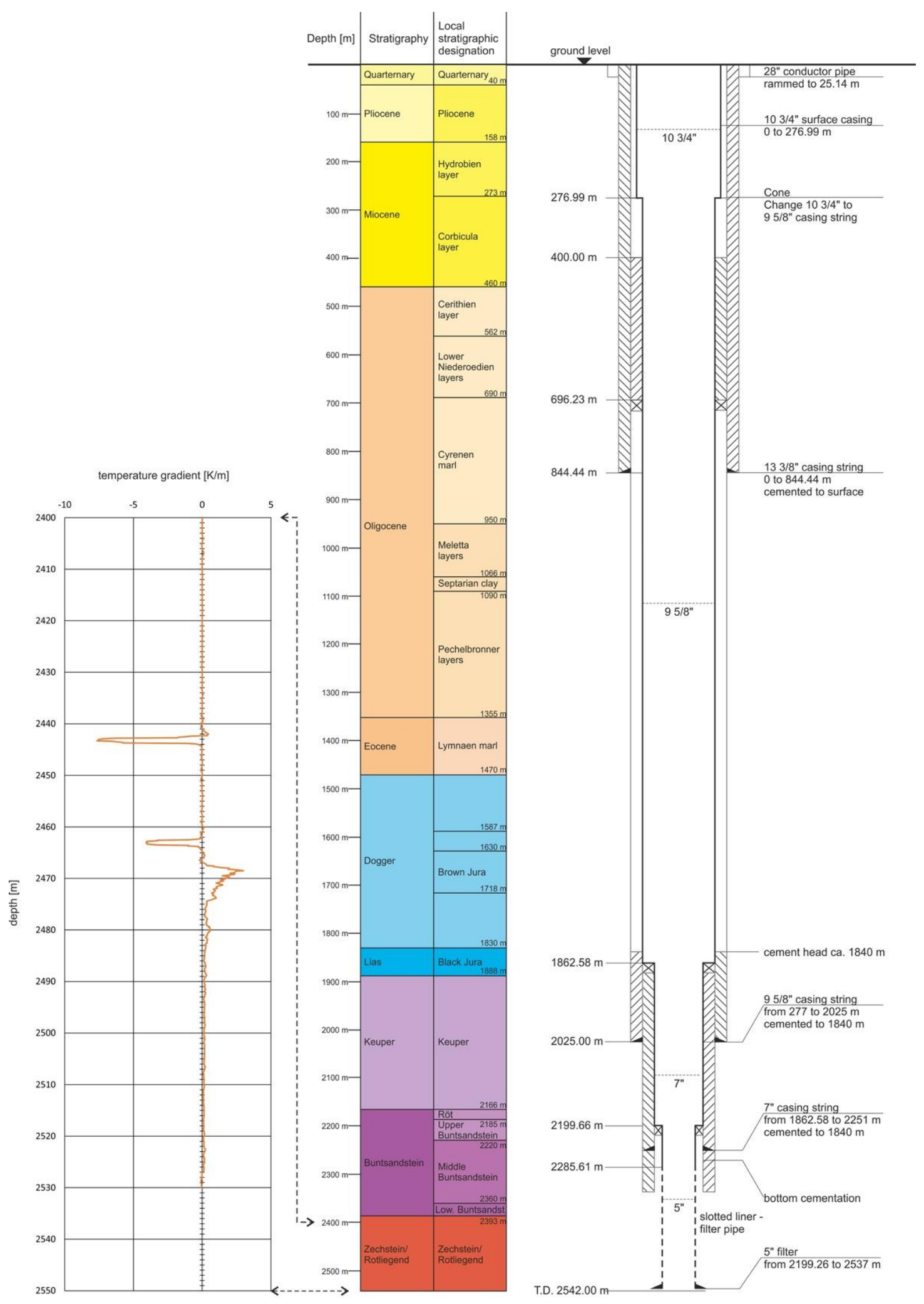

Fig. 5.2: Drilling profile and extension drawing of the GB2 well. The temperature gradient log of the reservoir section indicates two major water inflow zones. 


\subsubsection{Well testing and reservoir parameter}

Since the completion of the GB2 production well in 1985, a number of circulation tests were carried out. Based on the data from a well test in 2013, the average productivity index PI was determined to $10.7 \mathrm{~L} \mathrm{~s}^{-1} \mathrm{bar}^{-1}$ at a flow rate of $25 \mathrm{~L} \mathrm{~s}^{-1}$ and to $9.5 \mathrm{~L} \mathrm{~s}^{-1} \mathrm{bar}^{-1}$ at a flow rate of $29 \mathrm{~L} \mathrm{~s}^{-1}$. Further information about well tests including major reservoir parameters is documented in Table 5.2 (Bertleff et al., 1988; Fischer, 2010; Bartels, 2014).

Table 5.2: Analysis results of previous pumping tests perfomend in Bruchsal with resulting tranmissivity $(T)$ and storage coefficient $(S)$.

\begin{tabular}{lllll}
\hline Date & Duration (h) & Rates $\left(\mathrm{L} \mathrm{s}^{-1}\right)$ & $T\left(10^{-4} \mathrm{~m}^{2} \mathrm{~s}^{-1}\right)$ & $S\left(10^{-4}\right)$ \\
\hline March 1985 & 52 & 11.3 & 7.5 & 15,5 \\
April 2009 & 384 & $19-23$ & $2.1-4.2$ & -- \\
February/ March 2013 & 864 & $22-29$ & $\begin{array}{l}<9,1 \\
\text { (for laminar flow) }\end{array}$ & 9 \\
\hline
\end{tabular}

In the recent study, the aquifer test analysis tool AQTESOLV® of HydroSOLVE, Inc., version 4.50.002 professional, was employed. This software offers the option to determine the fracture length by applying the mathematical solution of Gringarten and Witherspoon (1972). For this purpose, the pressure data at pump depth was converted to reservoir pressure in order to eliminate the temperature effect on the water column. The resulting data set was density corrected by applying the approach described in Mao and Duan (2008).

In a second step, a data set from 2009 was used for a simultaneous plot of drawdown and logarithmic derivative (diagnostic plot, Fig. 5.3) in order to receive further information about the reservoir behaviour (Bourdet, 1983). The analysis of slope of the derivative can be used to develop a sitespecific conceptual model (Renard et al., 2009).

In early test phase, the slope of the derivative is 0.50 indicating a linear flow regime. In a second phase from $10^{4}$ to $2 \times 10^{5} \mathrm{~s}$ the averaged slope of the derivative tends to be 0.250 . Additional analysis of this phase using specialised plots provided by AQTESOLV® point to a bilinear flow regime at this stage. The final time period analysed presents zero slope, consequently reflecting a pseudo-radial or infinite acting radial flow (IARF).

The best curve fit of the data set was achieved by applying Gringarten-Witherspoon's approach for the analysis of a vertical fracture (Gringarten and Witherspoon, 1972). 


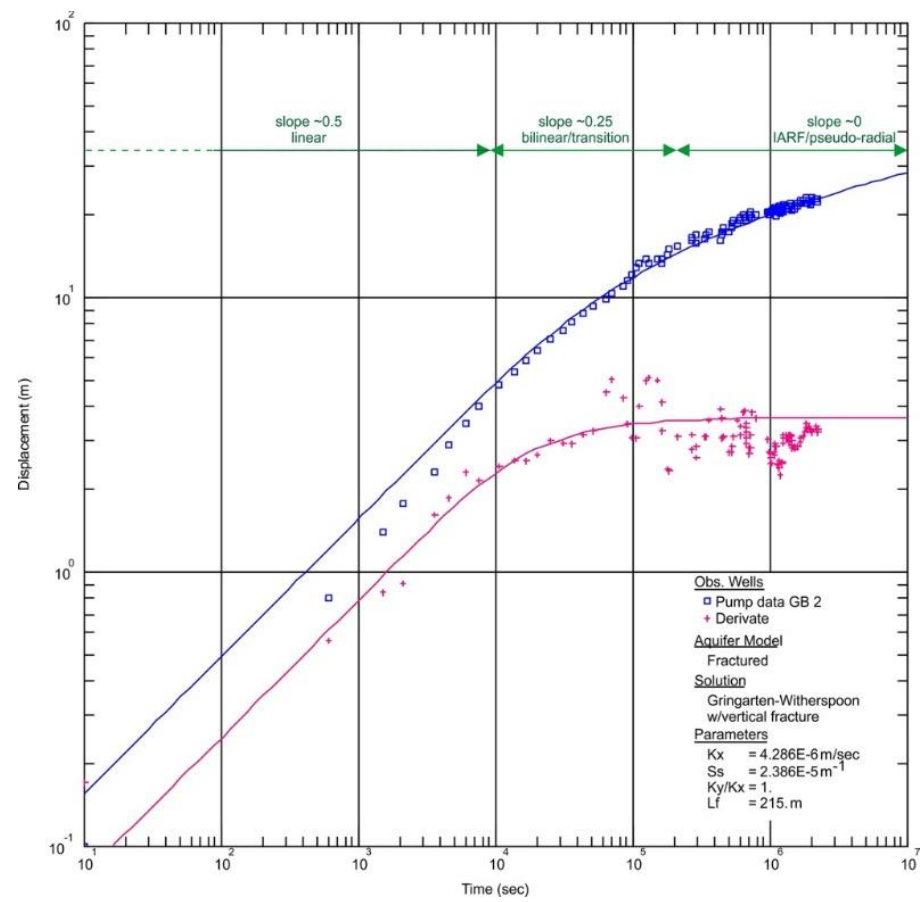

Fig. 5.3: Diagnostic plot of the Bruchsal drawdown data. The vertical axis represents both the drawdown and the logarithmic derivative.

The resulting hydraulic conductivity $(K x)$ and specific storage coefficient $(S s)$ are documented in Fig. 5.3 and are in agreement with previous studies mentioned above (cf. Table 5.2, aquifer thickness $\mathrm{m}=75 \mathrm{~m}$ ). The fracture length $L_{f}$ is determined to $215 \mathrm{~m}$.

\subsection{Conceptual model}

The model considers an idealized conductive fracture of width $w_{f}$, length, $L_{f}$ and height $h_{f}$ which is embedded in a porous media (Fig. 5.4). The fracture surface is presumed to be planar. Due to the very low hydraulic conductivity of the rock matrix, water flow is assumed to take place only in the fracture. The fracture is intersected by hydraulically inactive microfractures that are part of the rock matrix, forming pathways for ${ }^{222} \mathrm{Rn}$ to diffuse into the larger fractures. The following assumptions relating to the geometry and hydraulic properties of the system are made (Chen and Li, 1997):

(1) The length of the fracture is large relative to its opening width;

(2) Radionuclide concentration in the fracture is uniform perpendicular to the flow direction;

(3) Water flow in the fracture is laminar; 


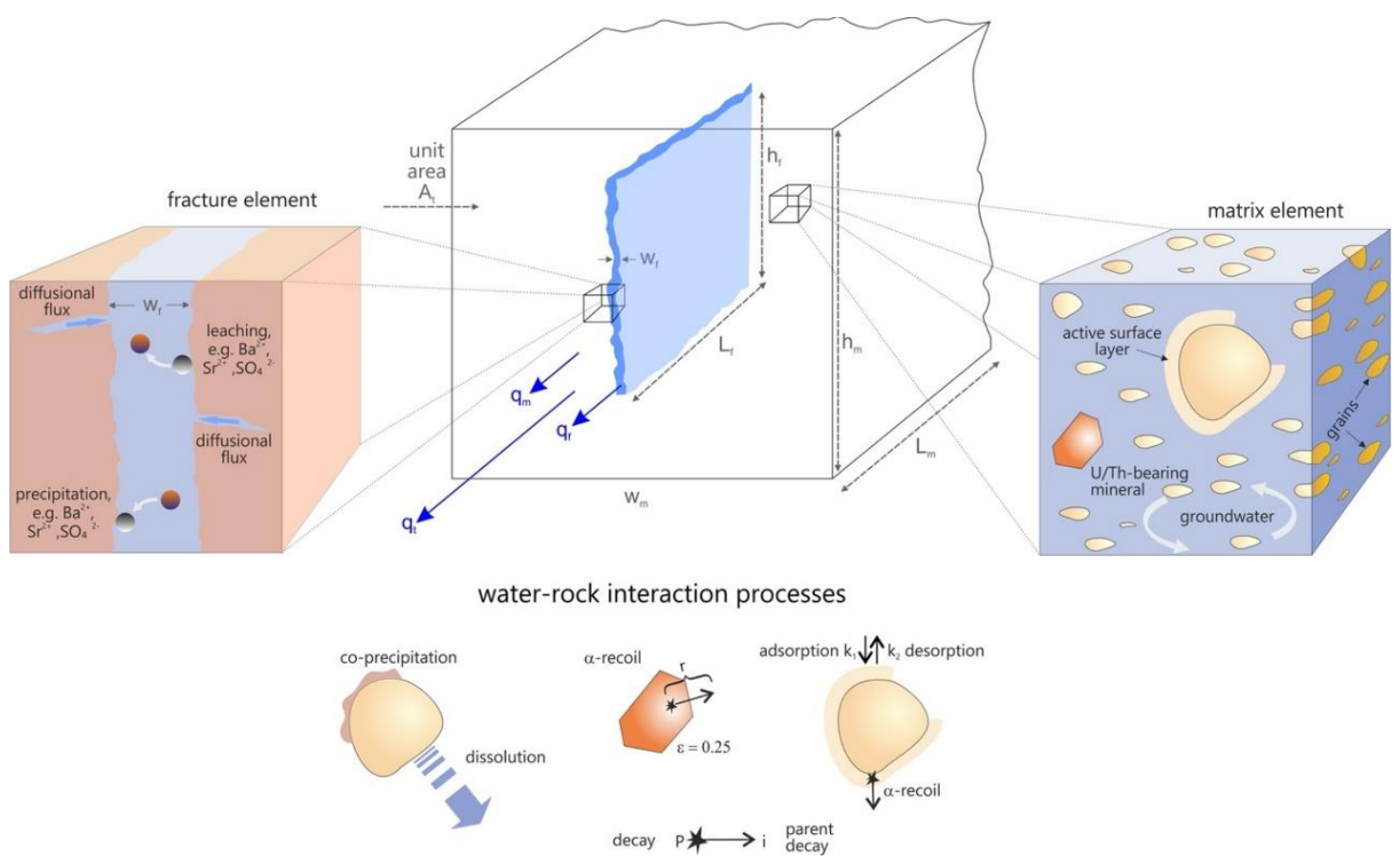

Fig. 5.4: Fluid flow model for the Bruchsal geothermal site: Fracture-dominated radionuclide transport within the reservoir section in consideration of the physico-chemical water-rock interactions (adsorption-desorption, dissolutionprecipitation, alpha recoil) which take place to varying extent in both the fracture (left cube) and matrix element (right cube).

(4) Radionuclide transport along the fracture is much faster than transport within the rock matrix

(5) Radionuclide transport within the porous matrix is only by molecular diffusion, perpendicular to the fracture axis.

From the first two assumptions, radionuclide transport takes place by one-dimensional flow along the fracture. The third assumption allows the use of Darcy's law. The last two assumptions lead to a mass flux within the rock matrix perpendicular to the fracture plane.

Apart from transport, ${ }^{238} \mathrm{U}$ series radionuclides are controlled by a number of physico-chemical processes including radioactive decay and growth, recoil supply, adsorption-desorption and precipitation-dissolution. Applying the water-rock interaction model for porous media suggested by $\mathrm{Ku}$ et al. (1992), radionuclides are considered to reside in three pools: the dissolved pool, adsorbed on rock surfaces, and the solid pool. The interrelationship between water-rock interaction processes and the three pools are summarized as follows (Ku et al., 1992):

(1) In the dissolved pool, radionuclides are exchangeable with those in the adsorbed pool, but not with those in the solid pool; 
(2) Transfer of radionuclides between dissolved and solid pools is achieved in particular by dissolution, co-precipitation and alpha recoil;

(3) Dissolution and precipitation are considered irreversible because timescales are not fast enough to maintain local equilibrium between the dissolved and solid pools;

(4) Alpha-recoil input from the adsorbed and dissolved pools to the solid pool is negligible;

(5) Distributions of radionuclides in solid, adsorbed, and dissolved pools remain stationary.

Since nuclide transport in the Bruchsal reservoir is considered to be fracture-dominated, supplementary assumptions were made:

(6) The hydraulically inactive pore spaces and microfractures, respectively, are the main source for alpha recoil, while recoil supply from large fractures may be negligible due to their relative low brine-rock interaction rates;

(7) Diffusion along microfractures is a necessary step in allowing the recoiled atoms to enter the flow system.

The model parameters are listed in Table 5.3.

Table 5.3: Parameters used in the water-rock interaction model.

\begin{tabular}{|c|c|c|}
\hline Symbol & Parameter & SI units \\
\hline$A$ & Specific activity of a dissolved radionuclide & $\mathrm{Bq} \mathrm{L}^{-1}$ \\
\hline$A^{*}$ & $\begin{array}{l}\text { Specific activity of a dissolved radionuclide originated from diffusional } \\
\text { flux }\end{array}$ & $\mathrm{Bq} \mathrm{L}^{-1}$ \\
\hline $\bar{A}$ & Specific activity of a radionuclide in solids & $\mathrm{Bq} \mathrm{kg}^{-1}$ \\
\hline$C$ & Concentration of a dissolved radionuclide & atoms $\mathrm{L}^{-1}$ \\
\hline$C_{a}$ & Concentration of an adsorbed radionuclide & atoms $\mathrm{L}^{-1}$ \\
\hline$D_{L}$ & Hydrodynamic dispersion coefficient & $\mathrm{m}^{2} \mathrm{~s}^{-1}$ \\
\hline$D_{m}$ & Molecular diffusion coefficient of a radionuclide & $\mathrm{m}^{2} \mathrm{~s}^{-1}$ \\
\hline$F_{i}$ & Diffusional flux of a nuclide into larger fractures & $\mathrm{Bq} \mathrm{m}^{-2}$ \\
\hline$\varepsilon_{i}$ & Recoil efficiency for nuclide $i$ & - \\
\hline$\phi$ & Fracture porosity & - \\
\hline$\phi^{*}$ & Porosity of the rock matrix & - \\
\hline$h_{f}$ & Fracture height & $\mathrm{m}$ \\
\hline$k_{p}$ & First-order precipitation rate constant & $\mathrm{s}^{-1}$ \\
\hline$K$ & Dimensionless distribution coefficient & - \\
\hline$L$ & Diffusion length & $\mathrm{m}$ \\
\hline
\end{tabular}




\begin{tabular}{|c|c|c|}
\hline Symbol & Parameter & SI units \\
\hline$L_{\text {eff }}$ & Effective diffusion length & $\mathrm{m}$ \\
\hline$L_{f}$ & Fracture length & $\mathrm{m}$ \\
\hline$\lambda$ & Radioactive decay constant of radionuclide & $\mathrm{s}^{-1}$ \\
\hline$P$ & Supply rate of radionuclide to brine & $\mathrm{Bq} \mathrm{L}^{-1}$ \\
\hline$P_{d}$ & Supply rate of radionuclide to brine by desorption & $\mathrm{Bq} \mathrm{L}^{-1}$ \\
\hline$P_{r}$ & Supply rate of radionuclide to brine by alpha recoil & $\mathrm{Bq} \mathrm{L} \mathrm{L}^{-1}$ \\
\hline$P_{r}^{*}$ & $\begin{array}{l}\text { Supply rate of radionuclide to brine by alpha recoil in consideration of } \\
\text { diffusional flux }\end{array}$ & $\mathrm{Bq} \mathrm{L} \mathrm{L}^{-1}$ \\
\hline$P_{w}$ & Supply rate of radionuclide to brine by dissolution process & $\mathrm{Bq} \mathrm{L}^{-1}$ \\
\hline$Q$ & Supply rate of radionuclide by water flow & $\mathrm{Bq} \mathrm{L}^{-1}$ \\
\hline$r_{i}$ & Recoil distance of nuclide $i$ & $\mathrm{~m}$ \\
\hline$R_{f}$ & Retardation factor due to adsorption and desorption & - \\
\hline$R_{f}^{*}$ & $\begin{array}{l}\text { Retardation factor due to precipitation as well as adsorption and desorp- } \\
\text { tion }\end{array}$ & - \\
\hline$\rho_{s}$ & Density of aquifer solid & $\mathrm{kg} \mathrm{m} \mathrm{m}^{-3}$ \\
\hline$S$ & Storage coefficient & - \\
\hline$S_{a}$ & Scavenging rate of radionuclide from brine by adsorption to rock surfaces & $\mathrm{Bq} \mathrm{kg}^{-1}$ \\
\hline$S_{p}$ & $\begin{array}{l}\text { Scavenging rate of radionuclide from brine by co-precipitation with min- } \\
\text { erals }\end{array}$ & $\mathrm{Bq} \mathrm{kg}^{-1}$ \\
\hline$S_{f v}$ & $\begin{array}{l}\text { Specific surface area of a fracture, expressed as area of solid per volume } \\
\text { of fluid contacting the solids }\end{array}$ & $\mathrm{m}^{2} \mathrm{~m}^{-3}$ \\
\hline$S_{\text {eff }}$ & Effective surface area of equivalent fracture & $\mathrm{m}^{2}$ \\
\hline$S_{\text {total }}$ & Specific surface area of equivalent fracture & $\mathrm{m}^{2} \mathrm{~m}^{-3}$ \\
\hline$t$ & Time & s \\
\hline$w_{f}$ & Fracture width & $\mathrm{m}$ \\
\hline$v_{f}$ & Advection velocity of fracture fluid & $\mathrm{m} \mathrm{s}^{-1}$ \\
\hline$x$ & Distance & $\mathrm{m}$ \\
\hline$\zeta$ & Variance from fracture width & - \\
\hline ' & Superscript referring to radioactive parent & - \\
\hline
\end{tabular}

\subsection{Governing equation}

Constant radionuclide activities along the flow line are reached when supply is at the same rate from both the dissolved radionuclide fraction in geothermal brine and the adsorbed fraction on surfaces. For very short-lived radionuclides (with large decay constant $\lambda$ ), constant activities are reached over short distances. For the long-lived radionuclides the distance is controlled by the precipitation rate constant $\left(k_{p}\right)$. 
Assuming a one-dimensional fracture with constant aperture and constant water velocity, the mass balance for a nuclide can be described by the advection-dispersion equation extended by supply and scavenging terms:

$$
\frac{d}{d t}\left(C+C_{a}\right)=D_{L} \frac{d^{2} C}{d x^{2}}-v_{f} \frac{d C}{d x}+P-k_{p} C-\lambda\left(C+C_{a}\right)
$$

where $P$ is the supply rate of the nuclide (atoms s$~^{-1} \mathrm{~L}^{-1}$ ), $v_{f}$ is the advection velocity in the fracture $\left(\mathrm{m} \mathrm{s}^{-1}\right)$ and $D_{L}$ is the hydrodynamic dispersion coefficient $\left(\mathrm{m}^{2} \mathrm{~s}^{-1}\right)$. Eq. (5.1) is found by Ku et al. (1992) in a slightly modified form since these workers have defined one dimensional flow of Th$\mathrm{U}$ series radionuclides in porous rather than fractured media.

Assuming a parallel-plate fracture, Taylor dispersion is the primary mechanism causing dispersion (Taylor, 1953; Aris, 1956). The Taylor dispersion coefficient of a parallel-plate fracture is (e.g. Fischer et al., 1979):

$$
D_{L, \text { Taylor }}=\frac{v_{f}^{2} w_{f}^{2}}{210 D_{m}}
$$

where $w_{f}$ is the fracture width $(\mathrm{m})$ and $D_{m}$ the molecular diffusion coefficient $\left(\mathrm{m}^{2} \mathrm{~s}^{-1}\right)$.

The production rate $P$ of the radionuclide to the fluid comprises the following mechanisms:

$$
P=P_{r}+P_{w}+\lambda^{\prime}\left(C^{\prime}+C_{a}^{\prime}\right)
$$

where $P_{r}, P_{w}$ and $\lambda\left(C^{\prime}+C_{a}^{\prime}\right)$ are the supply rates of the radionuclide (atoms $\mathrm{s}^{-1} \mathrm{~L}^{-1}$ ) by alpha recoil, mineral dissolution and in-situ decay of its parent. The separation between the adsorbed $\left(C_{a}\right)$ and dissolved $(C)$ nuclides through chemical exchanges may be described by the dimensionless distribution coefficient $K\left(=C_{a} / C\right.$ ) indicating the extent of retardation that a radionuclide might undergo during its transport in the fluid. Here, the retardation factor $R_{f}$ describes the flow rate of the fluid relative to the rate of migration of the radionuclide in the flow commonly defined as (Krishnaswami et al., 1982):

$$
R_{f}=\frac{C+C_{a}}{C}=1+\frac{C_{a}}{C}=1+K
$$

Combing Eq. (5.1), (5.2), (5.3) and (5.4), Eq. (5.1) can be rewritten as follows (Ku et al., 1992):

$$
\frac{R_{f}}{R_{f}^{*}} \frac{d C}{d t}=\frac{D_{L}}{R_{f}^{*}} \frac{d^{2} C}{d x^{2}}-\frac{v_{f}}{R_{f}^{*}} \frac{d C}{d x}+\frac{P}{R_{f}^{*}}-\lambda C
$$

with 


$$
R_{f}^{*}=R_{f}+\frac{k_{p}}{\lambda}
$$

where $R_{f}^{*}$ is the retardation factor due to precipitation as well as adsorption and desorption. Since $R_{f}^{*}$ will increase with decreasing decay constants, $R_{f}^{*}$ does not differ from $R_{f}$ for short-lived radionuclides.

\subsection{1 ${ }^{222} \mathrm{Rn}$ flux from a plane rock surface}

Since alpha recoil mechanism is most pronounced in the hydraulically inactive rock matrix, diffusion along pore spaces and microfractures is believed to supply recoiled ${ }^{222} \mathrm{Rn}$ atoms from the rock matrix into large conductive fractures where the sampled brine resides (Rama and Moore, 1984; Andrews et al., 1986). Here, microfractures serve as diffusion pathways.

If radon supply is only by alpha recoil, then ${ }^{222} \mathrm{Rn}$ content of fracture fluids might be calculated from the radon flux $\left(F_{\mathrm{R} n 2}\right)$ from the fracture surfaces and the fracture dimensions introduced in Fig. 5.4 (Andrews at el., 1986):

$$
A_{R n 2}=\frac{2 h_{f} L_{f} F_{R n 2}}{w_{f} h_{f} L_{f}}\left(1-e^{-\lambda t}\right)
$$

where $t$ is the fluid residence time in the fracture and $w_{f}, h_{f}$ and $L_{f}$ are the width (m), height (m) and length $(\mathrm{m})$ of the fracture. Since the half-life period of ${ }^{222} \mathrm{Rn}$ is about 3.83 days, ${ }^{222} \mathrm{Rn}$ reaches steady state activities within a short time $(\approx 30$ days) and Eq. (5.7) is then rewritten as follows:

$$
A_{R n 2}=\frac{2 F_{R n 2}}{w_{f}}
$$

However, since $\mathrm{R}$ a isotopes are largely dissolved in the Bruchsal brine, Eq. (5.8) has to be extended to ${ }^{222} \mathrm{Rn}$ supply due to the radioactive decay of ${ }^{226} \mathrm{Ra}$ dissolved in brine. Furthermore, ${ }^{222} \mathrm{Rn}$ may be also be produced by direct recoil from the large fracture surfaces, even though it has to be assumed that ${ }^{222} \mathrm{Rn}$ accumulation by this process is quite low due to the relative low water-rock interaction rates. Dissolution-precipitation can be negligible due to the short half-life of ${ }^{222} \mathrm{Rn}\left(t_{1 / 2}=3.83 \mathrm{~d}\right)$. Thus, ${ }^{222} \mathrm{Rn}$ steady state activity in a large, conductive fracture be described by the following mass balance equation (Ku et al., 1992):

$$
A_{R n 2}=R_{f 2}^{\prime} A_{R a 6}+\frac{2 F_{R n 2}}{w_{f}}+\frac{2 \bar{A}_{U 38} \varepsilon_{R n 2} r_{R n 2} \rho_{S}}{w_{f}}
$$

Eq. (5.9) can be rearranged to obtain the fracture width: 


$$
w_{f}=\frac{2 F_{R n 2}+2 \bar{A}_{U 38} \varepsilon_{R n 2} r_{R n 2} \rho_{S}}{A_{R n 2}-A_{R a 6} R_{f 2}^{\prime}}
$$

where the ${ }^{222} \mathrm{Rn}$ flux, $F_{\mathrm{Rn} 2}$, from planar rock surfaces (atoms $\mathrm{s}^{-1} \mathrm{~cm}^{-2}$ ) into the fracture fluid by diffusion can be expressed as (Ku et al., 1992):

$$
F_{R n 2}=-\phi^{*} D_{m}\left(\frac{d C}{d x}\right)_{x=0}=\phi^{*} \sqrt{\frac{D_{m}}{\lambda_{R n 2}}}\left(P_{r}+R_{f 1}^{\prime} A_{R a 6}-A_{R n 2}\right)
$$

where $\Phi^{*}$ is the microfracture porosity $0, D_{m}$ is the molecular diffusivity of radon $\left(\mathrm{m}^{2} \mathrm{~s}^{-1}\right), \lambda$ is the decay constant $\left(\mathrm{s}^{-1}\right), P_{r}$ is the supply rate from recoil (atoms $\left.\mathrm{s}^{-1} \mathrm{~L}^{-1}\right) \cdot \mathrm{R}_{f}$ is the retardation factor 0 and $A$ is the activity of the dissolved radionuclide (atoms s${ }^{-1} \mathrm{~L}^{-1}$ ) with superscript ( ${ }^{\zeta}$ referring to its radioactive parent.

Alpha recoil supply rates $\left(P_{r}\right)$ for ${ }^{222} \mathrm{Rn}$ can be estimated from the activities of the ${ }^{238} \mathrm{U}$ series progenitors in the adjacent rocks (e.g. Lou et al. 2000):

$$
P_{r}=\bar{A}_{R a 6} \varepsilon_{R n 2} r S_{f v} \rho_{s}
$$

where $\overline{A^{\prime}}$ is the parent activity in solids (atoms s$\left.{ }^{-1} \mathrm{~L}^{-1}\right), r$ is the recoil distance $(\AA), \varepsilon_{i}$ is the recoil efficiency for the nuclide $), S_{f v}$ is the flow-wetted surface area of solids $\left(\mathrm{m}^{2} \mathrm{~m}^{-3}\right)$ and $\rho_{s}$ is the density of solid $\left(\mathrm{kg} \mathrm{m}^{-3}\right)$.

\subsection{Results and discussion}

\subsubsection{Estimating in situ fracture width from ${ }^{222} \mathrm{Rn}$ flux $\left(w_{f}\right)$}

The ${ }^{222} \mathrm{Rn}$ flux from a plane fracture surface is largely due to diffusion through non-conductive microfractures. Since the diffusion length $\left(L=\sqrt{D_{m} / \lambda}\right)$ depends on the ${ }^{222} \mathrm{Rn}$ decay constant $\lambda_{\mathrm{Rn} 2}$ $\left(\mathrm{s}^{-1}\right)$ and the diffusion coefficient $D_{m}\left(\mathrm{~cm}^{2} \mathrm{~s}^{-1}\right),{ }^{222} \mathrm{Rn}$ diffusion length is about $2.18 \mathrm{~cm}$ in water (with $D_{m}=1.0 \times 10^{-5} \mathrm{~cm}^{2} \mathrm{~s}^{-1}$ according to Tanner, 1964).

Therefore, the ${ }^{222} \mathrm{Rn}$ activity in brine originating from diffusion processes is reduced by a factor of 0.37 over a distance equal to one diffusion length. Less than $5 \%$ reaches a distance equal to five diffusion lengths (Fig. 5.5).

Considering the site-specific rock matrix porosity of 0.05 (Joachim et al. 1987), the effective diffusion length of $222 \mathrm{Rn}$ $\left(L_{e f f}=\phi^{*} \sqrt{D_{m} / \lambda}\right)$ within the Bruchsal system is reduced to $0.02 \mathrm{~cm}$ (

Table 5.4). 


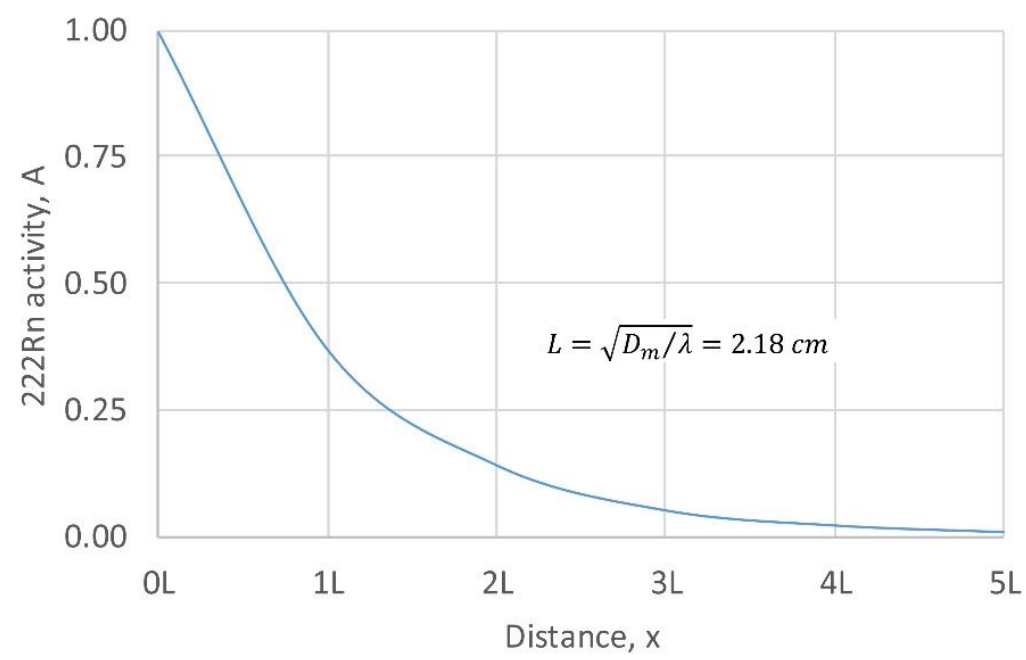

Fig. 5.5: ${ }^{222} \mathrm{Rn}$ diffusion length $(L)$ normalized to radon fluid activity.

Table 5.4: ${ }^{222} \mathrm{Rn}$ diffusional flux $\left(F_{\mathrm{R} n 2}\right)$ as a function of the effective diffusion length $\left(\phi^{*} \sqrt{D_{m} / \lambda}\right)$, radon production $\left(P_{\mathrm{R} n 2}\right)$ and scavenging rate $\left(S_{\mathrm{R} n 2}\right)$.

\begin{tabular}{|ccccc|}
\hline Decay & $\begin{array}{c}P_{\mathrm{R} n 2} \\
(\text { atoms L-1 s}\end{array}$ & $\begin{array}{c}S_{\mathrm{R} n 2} \\
\left(\text { atoms L } \mathrm{L}^{-1}\right)\end{array}$ & $\begin{array}{c}\phi^{*} \sqrt{D_{m} / \lambda} \\
(\mathrm{cm})\end{array}$ & $\begin{array}{c}F_{\mathrm{R} n 2} \\
(\text { atoms cm }\end{array}$
\end{tabular}

Applying Eq. (5.11), the ${ }^{222} \mathrm{Rn}$ flux is about $4.2 \times 10^{-3}$ atoms $\mathrm{s}^{-1} \mathrm{~cm}^{-2}$ whereas ${ }^{222} \mathrm{Rn}$ supply from alpha recoil $\left(P_{r}\right)$ is about 46 atoms s$~^{-1} \mathrm{~L}^{-1}$ calculated from the following parameters: Progenitor activity $\left(\overline{A^{\prime}}\right)$ in the site-specific reservoir rock is about ${ }^{238} \mathrm{U}=18.0 \mathrm{~Bq} \mathrm{~kg}^{-1}$ (Kölbel et al., 2020a). Recoil efficiency $\varepsilon_{R n 2}$ is 0.25 based on geometrical considerations (Kigoshi, 1971). Sun and Semkow (1998) suggested an average ${ }^{222} \mathrm{Rn}$ recoil distances in $800 \AA$ surface zone of quartz about 380.7 $\AA$ obtained from Monte Carlo simulations. Surface area is estimated to be $2000 \mathrm{~m}^{2} \mathrm{~kg}^{-1}$ for the Permo-Triassic sandstones (Heap et al., 2019). This corresponds to a flow wetted surface of $1.0 \mathrm{x}$ $10^{8} \mathrm{~m}^{2} \mathrm{~m}^{-3}$ based on a porosity of 0.05 .

Fig. 5.6 shows the relation between the radon activity, diffusion flux and fracture width. Referring to Eq. (5.11), the ${ }^{222} \mathrm{Rn}$ flux $\left(F_{\mathrm{R} n 2}\right)$ varies depending on ${ }^{222} \mathrm{Rn}$ activity measured in brine whereas $F_{R n 2}$ decreases with increasing radon activity. A reduction in the ${ }^{222} \mathrm{Rn}$ flux would proportionately reduce the fracture width. If the diffusional flux of radon remains constant, however, decreasing ${ }^{222} \mathrm{Rn}$ activities in the fluid would result in larger fracture width. Applying Eq. (5.10), the site-specific fracture width for the Bruchsal system is about $10 \mathrm{~mm}$ based on the average ${ }^{222} \mathrm{Rn}$ activity of 38 atoms $\mathrm{L}^{-1} \mathrm{~s}^{-1}$ (cf. Table 5.1). 


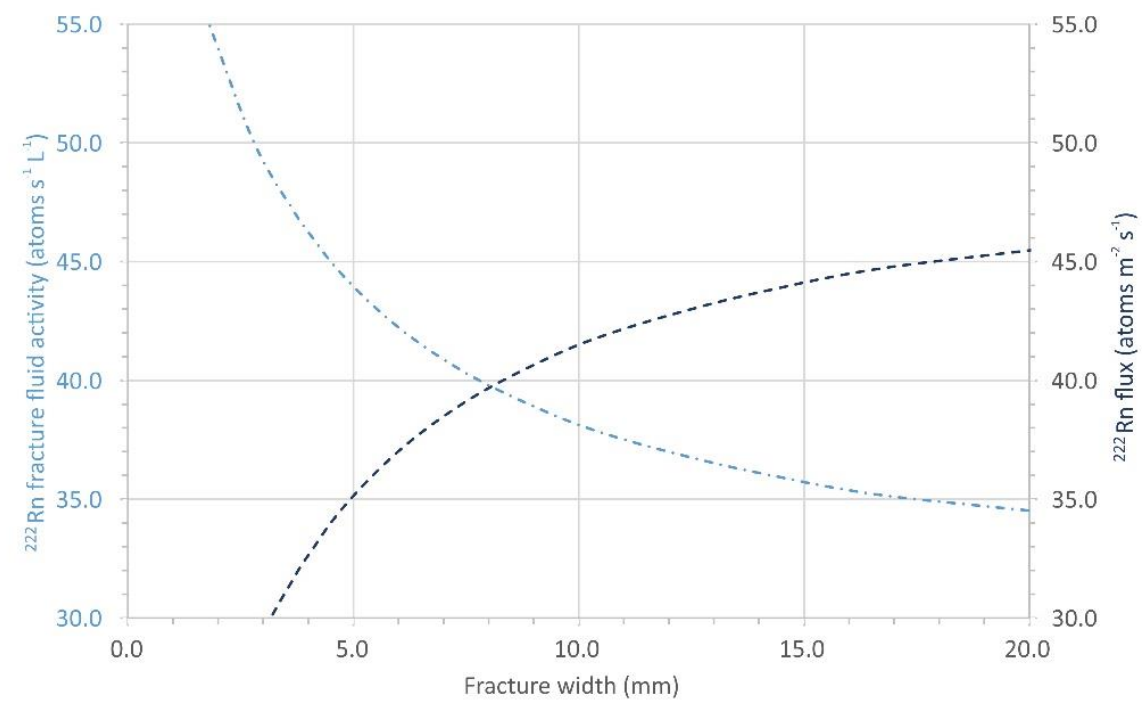

Fig. 5.6: Interaction between site-specific ${ }^{222} \mathrm{Rn}$ activity in the large fracture fluid (atoms s-1 $\mathrm{L}^{-1}$ ), diffusion flux $F_{\mathrm{R} n 2}$ (atoms s $\mathrm{m}^{-1} \mathrm{~m}^{-2}$ ) and fracture width $(\mathrm{mm})$.

\subsubsection{Estimating site-specific fracture length $\left(L_{f}\right)$}

Since most of the sampled brine resides in the larger fractures, fracture length can be derived by a mass balance approach for ${ }^{222} \mathrm{Rn}$. The ${ }^{222} \mathrm{Rn}$ activity of the fracture fluid may be expressed as follows:

$$
\frac{d A_{R n 2}}{d t}=D_{L} \frac{d^{2} A_{R n 2}}{d x^{2}}-v_{f} \frac{d A_{R n 2}}{d x}-\lambda_{R n 2} A_{R n 2}+\lambda_{R n 2} A_{R a 6}+\frac{2 F_{R n 2}}{w_{f}}=0
$$

where the first two terms describe the transport by dispersion and advection, respectively. The radioactive decay of ${ }^{222} \mathrm{Rn}\left(\lambda_{\mathrm{Rn} 2} A_{\mathrm{Rn} 2}\right)$ in the fracture fluid is balanced by radioactive decay of ${ }^{226} \mathrm{Ra}$ dissolved in brine $\left(\lambda_{R n 2} A_{R a b}\right)$ and by the diffusional flux of recoiled ${ }^{222} \mathrm{Rn}$ atoms from the rock matrix $\left(2 F_{\mathrm{R} n 2} / w_{f}\right)$. Retardation can be neglected since ${ }^{222} \mathrm{Rn}$ behaves like a conservative tracer.

While ${ }^{226} \mathrm{Ra}\left(t_{1 / 2}=1600 \mathrm{y}\right)$ has a much longer half-life than ${ }^{222} \mathrm{Rn}\left(t_{1 / 2}=3.83 \mathrm{~d}\right)$, the distance $\overline{x_{R} n}$ required for ${ }^{222} \mathrm{Rn}$ to reach steady-state activity is primarily controlled by the distance $\overline{x_{\text {Ra }}}$ required for ${ }^{226} \mathrm{Ra}$ to reach a constant activity. Thus, the parameter $\overline{x_{\mathrm{R}} 6}$ is the length scale for transport and determines the distance required so that $\delta C / \delta x \approx 0$. Appling Eq. (5.1) to the long-lived ${ }^{226} \mathrm{Ra}$, the time-dependent evolution of its activity may be expressed as:

$$
\frac{d A_{R a 6}}{d t}=\frac{D_{L}}{R_{f}} \frac{d^{2} A_{R a 6}}{d x^{2}}-\frac{v_{f}}{R_{f}} \frac{d A_{R a 6}}{d x}+\frac{\lambda_{R a 6} P_{R a 6}}{R_{f}}-\frac{k_{p} A_{R a 6}}{R_{f}}-\lambda_{R a 6} A_{R a 6}=0
$$


with the production rate

$$
P_{R a 6}=P_{r}+P_{w}+\lambda^{\prime}\left(C^{\prime}+C_{a}^{\prime}\right)
$$

The ${ }^{226} \mathrm{Ra}$ generation is mainly from recoil $\left(P_{r}\right)$ and mineral dissolution $\left(P_{w}\right)$, while production from the dissolved parent $\lambda^{\prime}\left(C^{\prime}+C_{a}^{\prime}\right)$ can be neglected since ${ }^{230} \mathrm{Th}$ is quite insoluble in deep, reducing brines (e.g. Osmond and Cowart, 1982; Zukin et al., 1987). Since ${ }^{226} \mathrm{Ra}$ has a half-life of 1600 years, it is susceptible for time-consuming water-rock interaction processes such as mineral dissolution and co-precipitation. Therefore, the model equation for ${ }^{226} \mathrm{Ra}$ is much more complex than for ${ }^{222} \mathrm{Rn}$. The behavior of ${ }^{226} \mathrm{Ra}$ in the Bruchsal reservoir was investigated previously by Kölbel et al. (2020a) and Kölbel et al. (2020b). The relevant water-rock interaction rates for ${ }^{226} \mathrm{Ra}$ are listed in Table 5.5 .

Table 5.5: Model derived production and scavenging rates (atoms $\mathrm{L}^{-1} \mathrm{~s}^{-1}$ ) for ${ }^{226} \mathrm{Ra}$ in the Bruchsal geothermal system. Data published in Kölbel et al. (in preparation).

\begin{tabular}{lcc}
\hline Water-rock interaction processes & & 226Ra \\
\hline Supply from alpha recoil in consideration of the & $P_{r}^{*}$ & 244 \\
diffusional flux & $P_{d}$ & 1,268 \\
Production from desorption & $P_{w}$ & 72,391 \\
Production from dissolution & $S_{a}$ & 1,276 \\
Scavenging from adsorption & $S_{p}$ & 72,585 \\
Scavenging from precipitation & $Q^{2}$ & -13 \\
Supply by water flow & $A_{\mathrm{Ra}}$ & 29 \\
\hline \hline Steady state activity &
\end{tabular}

The retardation factor $R_{f}$ of ${ }^{226} \mathrm{Ra}$ is assumed to be 1.3 estimated from the ${ }^{222} \mathrm{Rn} /{ }^{226} \mathrm{Ra}$ fluid activity ratio. Eq. (5.13) and (5.14) are numerically approximated by finite difference techniques performed in Mathcad ${ }^{\circledR}$. Site-specific model parameters are listed in Table 5.6.

Table 5.6: Site-specific input parameters for the Bruchsal system.

\begin{tabular}{llll}
\hline Parameter & Symbol & Unit & Value \\
\hline Fracture width & $w_{f}$ & $\mathrm{~m}$ & 0.01 \\
Fracture porosity & $\phi$ & 0 & 0.01 \\
Mean velocity & $v_{f}$ & $\mathrm{~m} \mathrm{~s}^{-1}$ & $2.0 \times 10^{-6}$ \\
${ }^{222} \mathrm{Rn}$ dispersion coefficient & $D_{L}$ & $\mathrm{~m}^{2} \mathrm{~s}^{-1}$ & $1.9 \times 10^{-9}$ \\
${ }^{222} \mathrm{Rn}$ diffusion coefficient & $D_{m}$ & $\mathrm{~m}^{2} \mathrm{~s}^{-1}$ & $1.0 \times 10^{-9}$ \\
\hline
\end{tabular}

Note that fracture length is sensitive to the advection velocity, $v_{f}$. The evolution of the ${ }^{222} \mathrm{Rn}$ activity 
for the averaged advection velocity $\pm 25 \%$ is displayed in Fig. 5.7. The distance $\bar{x}_{R n}$ which is required to reach steady-state conditions for ${ }^{222} \mathrm{Rn}$ increases with increasing flow velocity. Thus, greater fracture lengths result from higher flow velocities. Fracture length $L_{f}$ ranges between 190 and $320 \mathrm{~m}$. Site-specific fracture length is about $250 \mathrm{~m}$ for a mean velocity of $2.0 \times 10^{-6} \mathrm{~m} \mathrm{~s}^{-1}$.

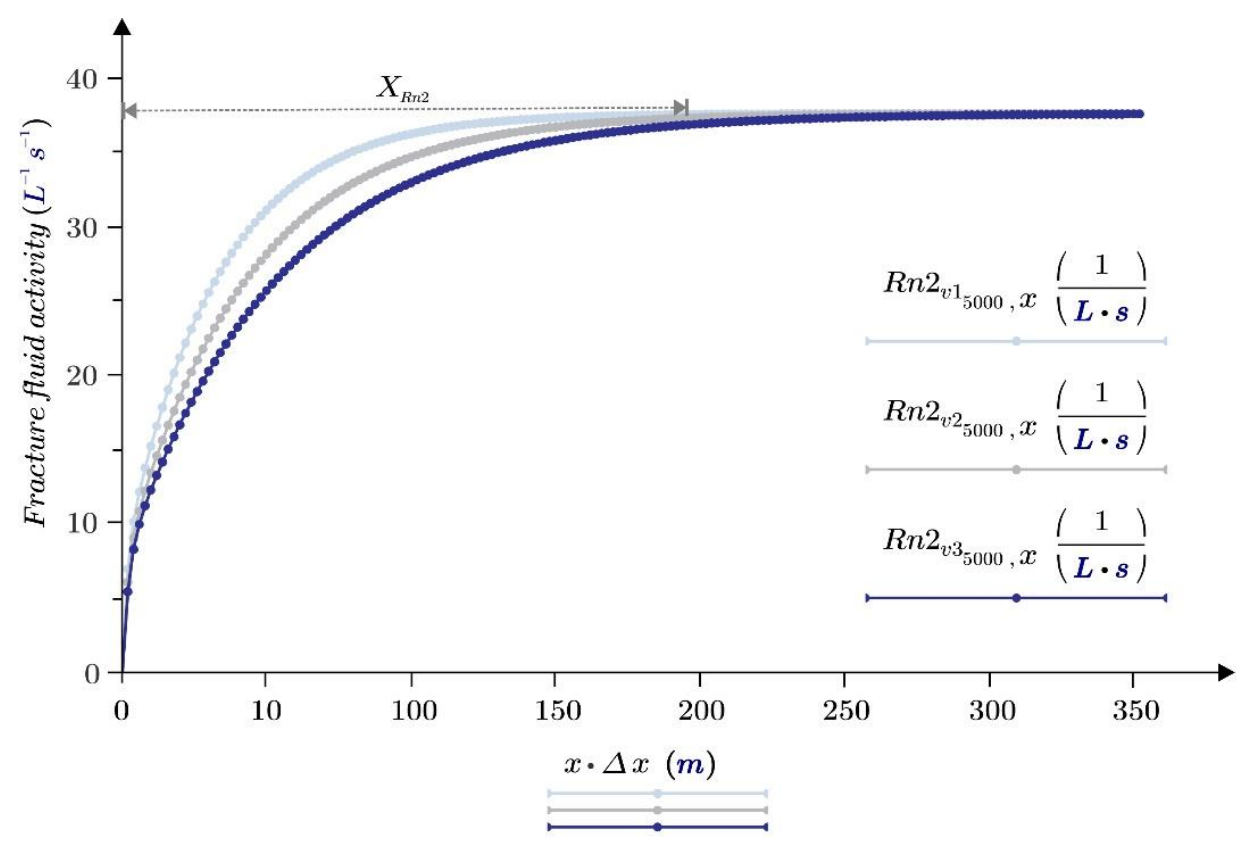

Fig. 5.7: ${ }^{222} \mathrm{Rn}$ fracture fluid activity as a function of distance $x(\mathrm{~m})$ for a mean flow velocity of $2.0 \times 10^{-6} \mathrm{~m} \mathrm{~s}^{-1} \pm 25$ $\%$.

This fracture length is in agreement with the fracture length determined by means of AQTESOLV ${ }^{\circledR}$ using the mathematical solution of Gringarten and Witherspoon (1972) (cf. Fig. 5.3). In Fig. 5.8 various storage coefficients $S O$ are compared with the related fracture length $L_{f}(\mathrm{~m})$ calculated following the Gringarten-Witherspoon approach. The relation between both parameters can be described by the function ( $L_{f}^{2} \times S \approx$ const.). This points to a certain ambiguity of the results of the analysis. Since $L_{f}=\sqrt{\text { const. } / S}$, it appears that the determination of the fracture length strongly depends on exact values of the storage coefficient. In addition, it becomes visible that already within one order of magnitude of the storage coefficient the resulting fracture length may differ by several hundred meters. This underlines the importance of high-quality data as well as a thorough data analysis.

The well testing data from Bruchsal originate from 1985, 2009 and 2013. It might appear that due to technical improvements of the data recording systems the younger data allow more accurate analysis. This assumption, however, is not supported by this study, because the lowest and highest determined storage coefficients result from the more recent data sets, while the mean values are calculated from the 1985 data. 


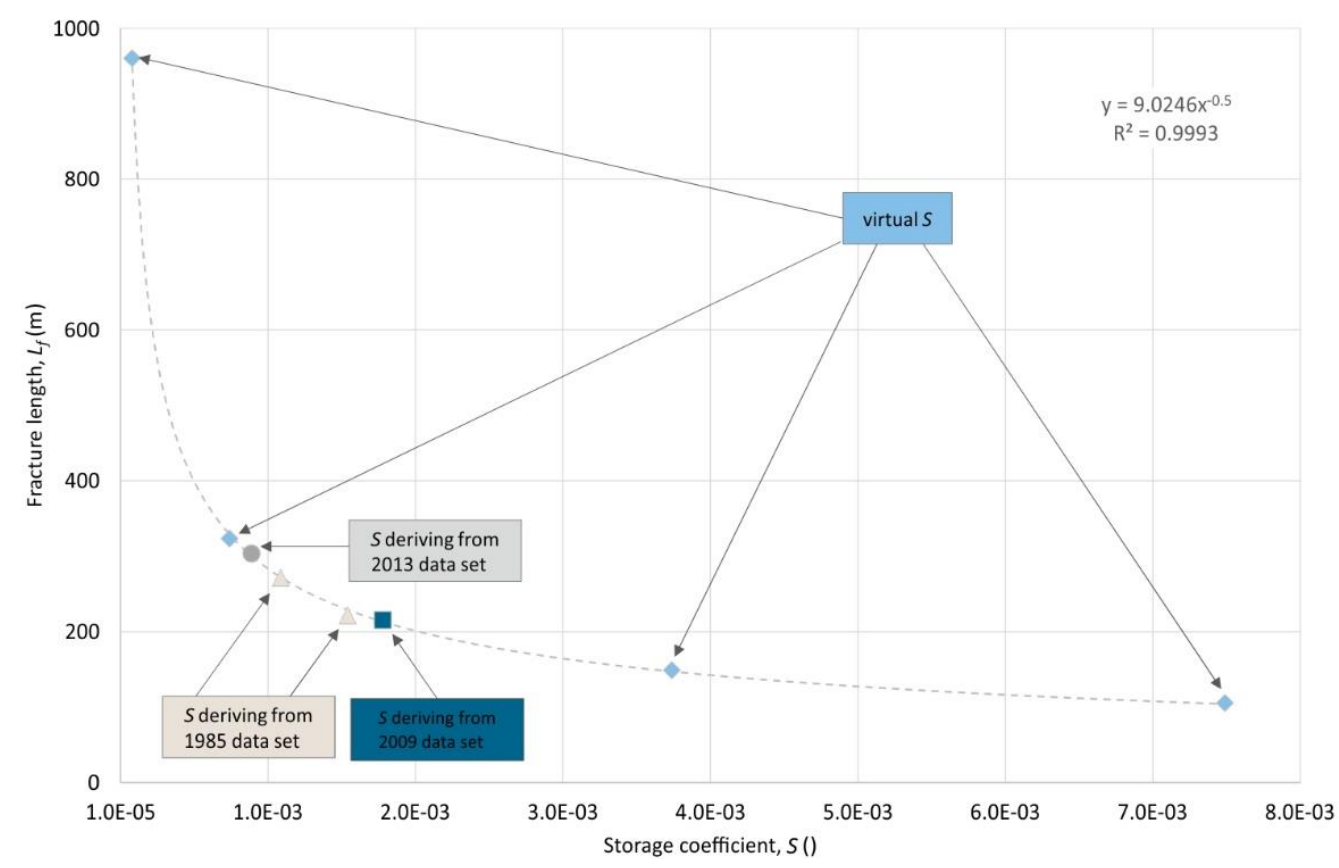

Fig. 5.8: Calculated fracture length $L_{f}(\mathrm{~m})$ vs. storage coefficients $S O$ in the range from $9 \times 10^{-5}$ to $7.5 \times 10^{-3}$.

The storage coefficients calculated previously based on GB2 pumping test data vary between $9.0 \mathrm{x}$ $10^{-4}$ to $15.5 \times 10^{-4}$ O (Bertleff et al., 1988; Fischer, 2010; Bartels, 2014) (cf. Table 5.2) and result in fracture lengths between 225 and $300 \mathrm{~m}$. The value calculated in this study $\left(S=18.0 \times 10^{-4}\right)$ is in line with the previous results and provides a fracture length of approximately $215 \mathrm{~m}$.

\subsubsection{Estimating fracture surface area $\left(S_{f v}\right)$}

The specific surface area $S_{f v}\left(\mathrm{~m}^{2} \mathrm{~m}^{-3}\right)$, also called flow wetted surface area, is defined as the surface area of solids per volume of fracture fluid contacting the solids. Using this definition of $S_{f v}$ and applying it to a fracture results in the following equation

$$
S_{f v}=\frac{A_{\text {surface }}}{V_{\text {frac fluid }}}=\frac{\left(n_{f} A\right) 2\left(w_{f}+h_{f}\right) L_{f}}{\left(n_{f} A\right) w_{f} h_{f} L_{f}}=2\left(\frac{1}{h_{f}}+\frac{1}{w_{f}}\right)
$$

where $\left(n_{f} A\right)$ is the number of fractures per block. Since the fracture width is much smaller than fracture height, then $1 / w_{f}>>1 / h_{f}$, and Eq. (5.15) simplifies to:

$$
S_{f v}=\frac{2}{w_{f}}
$$

Thus, the specific surface area of the fracture $S_{f v}$ is inversely proportional to the fracture width, $w_{f}$ (Fig. 5.9). 


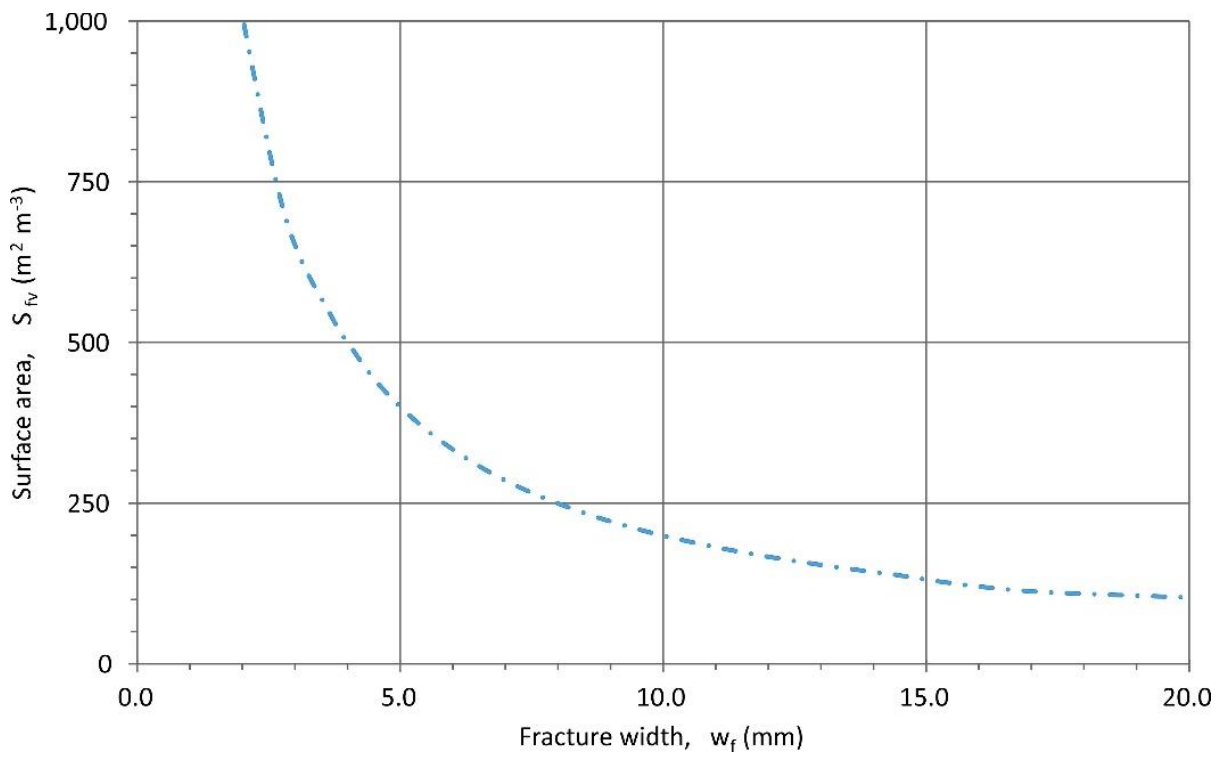

Fig. 5.9: Surface area of the equivalent fracture $\left(S_{f v}\right)$ for varying fracture widths $\left(w_{f}\right)$.

Since the fluid samples are mostly collected from the larger conductive fractures, the fracture width of the Bruchsal system, $w_{f}$, represents an averaged equivalent fracture width. This allows the use of an equivalent fracture approximating the behavior of the naturally fractured system.

Assuming that the Bruchsal system consists of two main fractures dominating the fluid flow in the near field of the production well as is indicated by the temperature gradient log (cf. Fig. 5.2), then the ratio of the single surface area $S_{f, 1}\left(S_{f, 2}\right)$ to the surface area of the equivalent fracture, $S_{\text {total }}$, can be expressed as

$$
\begin{aligned}
\frac{S_{f v, 1}}{S_{\text {total }}} & =\frac{\frac{2}{w_{f, 1}}}{\frac{2}{w_{f, 1}}+\frac{2}{w_{f, 2}}} \\
\frac{S_{f v, 2}}{S_{\text {total }}} & =\frac{\frac{2}{w_{f, 2}}}{\frac{2}{w_{f, 1}}+\frac{2}{w_{f, 2}}}
\end{aligned}
$$

Since $S_{f v}$ is inversely proportional to $w_{f}$, smaller fracture widths correspond to larger specific surface areas and vice versa.

If it is assumed that both main fractures of the Bruchsal system vary in their fracture widths and both differ from the equivalent fracture width by the same factor $(1 \pm \zeta)$, the following applies (Renner, 1996):

$$
w_{f 1}=(1+\zeta) w_{f}
$$




$$
w_{f 2}=(1-\zeta) w_{f}
$$

with $\zeta=0$ for two identical fractures. The larger the values for $\zeta$, the more the variance from the averaged equivalent fracture width and the greater the specific surface area of the equivalent fracture, $S_{\text {total }}$ (Fig. 5.10). Assuming two identical fractures with a mean fracture width of $10 \mathrm{~mm}$, the specific surface area of the fracture is approximately $400 \mathrm{~m}^{2} \mathrm{~m}^{-3}$. If $\zeta=0.5, S_{\text {total }}$ will be doubled.

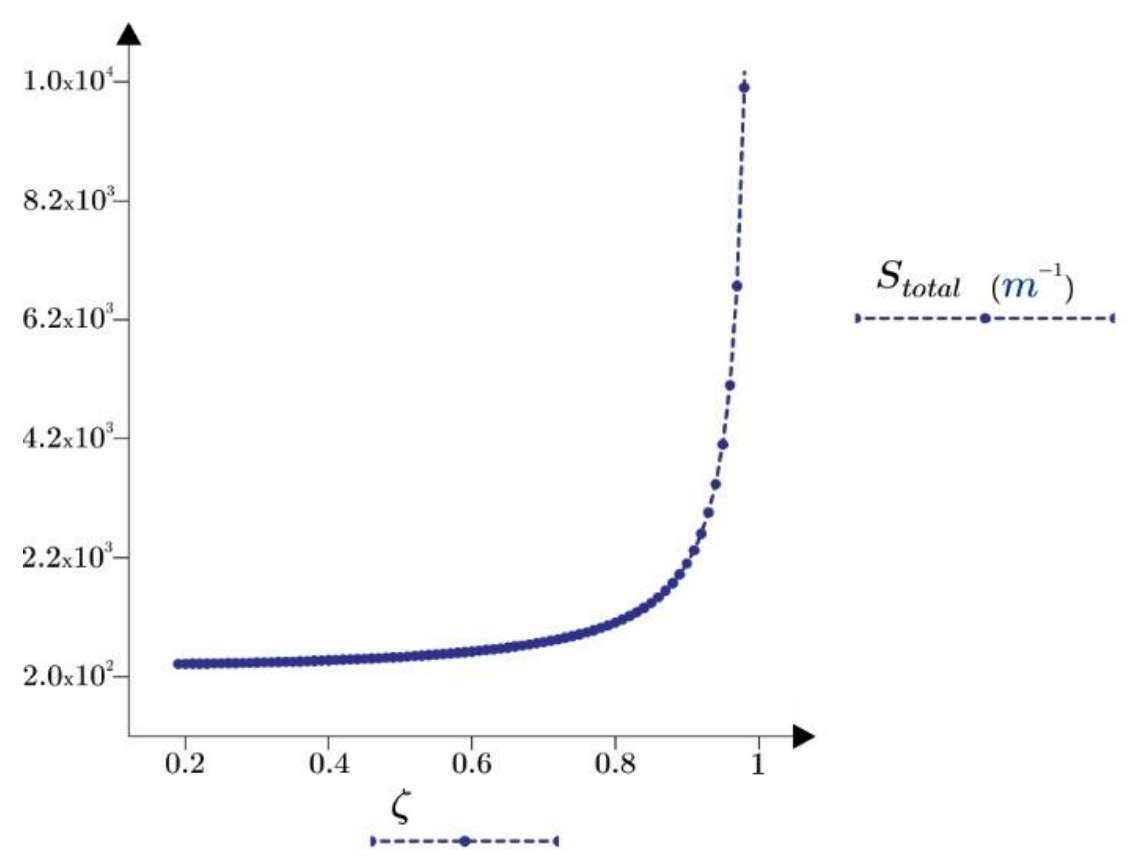

Fig. 5.10: Specific surface area of the equivalent fracture as a function of $\zeta$, the variance from the averaged equivalent fracture width.

The effective surface area of the equivalent fracture may be derived by fracture length $L_{f}(\mathrm{~m})$ and fracture height $h_{f}(\mathrm{~m})$ :

$$
S_{\text {eff }}=2 h_{f} L_{f}
$$

Since the fracture length is known from the one-dimensional transport modeling of ${ }^{222} \mathrm{Rn}$ as well as from Gringarten-Witherspoon analysis and fracture height $h_{f}$ may be derived from the temperature gradient $\log$ (cf. Fig. 5.2). Half of the peak widths correspond to a fracture height of $2.0 \pm 0.5$ $\mathrm{m}$ whereas the uncertainties refer to the inaccuracy of the measurements (e.g. tool speed, cable strain). Applying Eq. (5.19), the effective surface area is about $1000 \pm 250 \mathrm{~m}^{2}$ based on a mean fracture length of $250 \mathrm{~m}$. Note that $S_{\text {eff }}$ might be a lower limit since the fracture height $\left(h_{f}\right)$ is an apparent fracture height because of the dip of the Permo-Triassic formations. 
The corresponding fracture volume is about $5 \mathrm{~m}^{3}$ for the averaged equivalent fracture width of 10 $\mathrm{mm}$. Rearranging Eq. (5.8), the ${ }^{222} \mathrm{Rn}$ flux is the quotient of ${ }^{222} \mathrm{Rn}$ fluid activity $\left(A_{\mathrm{Rn} 2}^{*}\right)$ and the flow wetted surface area $S_{f i}$ :

$$
F_{R n 2}=\frac{A_{R n 2}^{*}}{S_{f v}}
$$

Note that $A_{\mathrm{R} n 2}^{*}$ is only the partition of ${ }^{222} \mathrm{Rn}$ that is produced by diffusional flux and not the average ${ }^{222} \mathrm{Rn}$ fluid activity since most of the ${ }^{222} \mathrm{Rn}$ activity is supplied by the radioactive decay of its dissolved ${ }^{226} \mathrm{Ra}$ parent. From Eq. (5.9) it becomes obvious that most of the ${ }^{222} \mathrm{Rn}$ activity is supplied by the radioactive decay of its dissolved ${ }^{226} \mathrm{R}$ a parent rather than by diffusion from recoiled atoms along grain boundaries in rock matrices and through microfractures. ${ }^{222} \mathrm{Rn}$ production from the latter is about $A_{\mathrm{R} n 2}^{*}=8.2 \mathrm{~Bq} \mathrm{~L}^{-1}$. Since the larger conductive fracture has relative low water-rock interaction rates, the effect of direct alpha recoil from the fracture surfaces is negligible.

Since one unit volume of fracture fluid contains 8200 atoms s${ }^{-1}$ of ${ }^{222} \mathrm{Rn}$, large fracture lengths enforce small values for fracture height at a constant fracture width. For $L_{f}=250 \mathrm{~m}$ and $w_{f}=10$ $\mathrm{mm}, h_{f}$ has to be $0.4 \mathrm{~m}$ to obtain a unit volume of fracture fluid. Five times the unit volume corresponds to the determined fracture height of $2 \mathrm{~m}$ and the associated fracture volume of $5 \mathrm{~m}^{3}$.

\subsection{Conclusions}

In this study we introduced an integrative approach for the estimation of site-specific fracture dimensions applied on the Bruchsal geothermal system which is located at the eastern main boundary of the Upper Rhine Graben.

Hydraulic reservoir parameters were obtained from well testing data. Evaluation of the flow regime indicates a fracture dominated fluid flow without further matrix support allowing us to use the concept of an equivalent fracture for estimating site-specific fracture dimensions by means of the inert gas ${ }^{222} \mathrm{Rn}$.

The ${ }^{222} \mathrm{Rn}$ activity in the Bruchsal fluid is on average 38 atoms s${ }^{-1} \mathrm{~L}^{-1}$. Its supply is mainly from radioactive decay of dissolved ${ }^{226} \mathrm{Ra}(80 \%)$. The remaining part is introduced via recoil in a network of nanopores of nearly non-conductive rock matrices. Direct recoil input from the surface of the larger fractures can be neglected due to its relative low water-rock interaction rates.

Diffusional flux through pore spaces and microfractures acting as diffusion pathways is believed to supply ${ }^{222} \mathrm{Rn}$ in larger conductive fractures in which the sampled brine resides. The ${ }^{222} \mathrm{Rn}$ flux from the fracture surfaces is about $4.2 \times 10^{-3}$ atoms $\mathrm{s}^{-1} \mathrm{~cm}^{-2}$. Estimates of the mean fracture width is about $10 \mathrm{~mm}$. 
Fracture length, $L_{f}$, is estimated by means of a mass balance approach for ${ }^{222} \mathrm{Rn}$ and ${ }^{226} \mathrm{Ra}$, respectively. The resulting fracture lengths depend on the advection velocity used for the calculation. Sitespecific fracture length is about $250 \mathrm{~m}$ for a mean velocity of $2.0 \times 10^{-6} \mathrm{~m} \mathrm{~s}^{-1}$. This fracture length is in acceptable agreement with the fracture length of $215 \mathrm{~m}$ determined by means of AQTESOLV ${ }^{\circledR}$ using the mathematical solution of Gringarten-Witherspoon for infinite flow in vertical fractures. Note that the fracture length is related to the specific storage coefficient where the relation between both parameters can be described by the function ( $L_{f}^{2} \times S \approx$ const.).

The specific surface area $S_{f v}$ of the fracture is inversely proportional to the fracture width, $w_{f}$. Since the fluid samples are mostly collected from the larger conductive fractures, $w_{f}$ represents an averaged equivalent fracture width. Therefore, in terms of $S_{f i}$, it makes a difference whether the geothermal brine is channeled by one large fracture or by several smaller fractures. Moreover, $S_{f v}$ will increase with increasing variance from the averaged equivalent fracture width.

With regard to reservoir engineering, it might be interesting to solve this question more detailed. Based on the determination of the averaged equivalent aperture calculation as presented in this paper, additional numerical modelling of heat transfer might a promising option to solve these issues.

\section{Acknowledgements}

This research was carried out within the framework of the ANEMONA project, funded by the German Federal Ministry for Economic Affairs and Energy (BMWi). We are also grateful to Dr. J. Bartels (AQUASOIL, Berlin) for stimulating discussions.

\section{References}

Andrews J.N., Hussain N., Batchelor A.S., Kwakwa K., 1986. ${ }^{222}$ Rn solution by the circulating fluids in a 'hot dry rock' geothermal reservoir. Appl. Geochim. 1, 647-657.

Andrews, J.N., Ford, D. J., Hussain, N., Trevedi, D., Youngman, M. J., 1989. Natural radioelement solution by circulating groundwaters in the Stripa granite. Geochimica et Cosmochimica Acta, 53, 1791-1802.

Bartels, J., 2014. Bohrungs- und reservoirhydraulische Auswertung des Stufenpumpversuchs vom 05.02. bis 18.03.2013. OSGa Final report, Karlsruhe.

Bertleff, B., Joachim, H., Koziorowski, G., Leiber, J., Ohmert, W., Prestel, R., Stober, I., Strayle, G., Villinger, E., Werner, J., 1988. Ergebnisse der Hydrogeothermiebohrungen in Baden-Wuerttemberg. Jh. Geol. Landesamt 
Bad.-Wuertt., 30, 27-116, Freiburg.

Bourdet D., Whittle T.M., Douglas A.A., Pirard Y.M., 1983. A new set of type curves simplifies well test analysis. World Oil 196:95-106.

Carvalho, F., Chambers, D., Fesenko, S., Moore, W.S., Porcelli, D., Vandenhove, H., Yankovich, T., 2014. Environmental pathways and corresponding models. In: The Environmental Behaviour of Radium: Revised Edition. Technical Reports Series No. 476, pp. 106-172. International Atomic Energy Agency, Vienna.

Chen, C.-T., Li, S.-H., 1997. Radionuclide transport in fractured porous media - Analytical solutions for a system of parallel fractures with a constant inlet flux. Waste Management 17, 53-64.

Condomines, M., Gourdin, E., Gataniou, D., Seidel, J.-L., 2012. Geochemical behaviour of Radium isotopes and Radon in a coastal thermal system (Balaruc-les-Bains, South of France). Geochimica et Cosmochimica Acta 98, $160-176$.

Eggeling, L., Genter, A., Kölbel, T., Münch, W., 2013. Impact of natural radionuclides on geothermal exploitation in the Upper Rhine Graben. Geothermics 47, 80-88.

Ferry C., Richon P., Beneito A., Cabrera J., Sabroux J.C, 2002. An experimental method for measuring the radon-222 emanation factor in rocks. Radiation Measurement, 35, 579-583.

Fischer T., 2010. Hydraulische Versuche unter geothermalen Bedingungen am Beispiel der Geothermieanlage Bruchsal im Rahmen des LOGRO-Projekts. Unpublished diploma thesis, Karlsruhe Institute of Technology (KIT), Karlsruhe.

Grant, M.A., Bixley, P.F., 2011. Geothermal Reservoir Engineering, Elsevier, Oxford.

Gringarten, A.C., Witherspoon, P.A., 1972. A method of analyzing pump test data from fractured aquifers, Int. Soc. Rock Mechanics and Int. Assoc. Eng. Geol., Proc. Symp. Rock Mechanics, Stuttgart, Vol. 3B, 1-9.

Hammond, D.E., Leslie, B.W., Ku., T.L., 1988a. ${ }^{222}$ Rn concentrations in deep formation waters and the geohydrology of the Cajon Pass borehole. Geophysical Research Letters 15 (9), 1045-1048.

Hammond, D.E., Zukin, J.G., Ku, T.L., 1988b. The kinetics of radioisotope exchange between brine and rock in a geothermal system. J. Geophys. Res. 93 (13), 175-186.

Heap, M.J., Villeneuve, M., Kushnir, A.R.L., Farquharson, J.I., Baud, P., Reuschlé, T., 2019. Rock mass strength and elastic modulus of the Buntsandstein: An important lithostratigraphic unit for geothermal exploitation in the Upper Rhine Graben. Geothermics 77, 236-256.

Joachim, H., Koziorowski, G., Leiber, J., 1987. Geothermiebohrungen Bruchsal 1a und 2, in: Maus, H. (Ed.), Jahreshefte des Geologischen Landesamtes Baden-Württemberg 29. Herder, Freiburg i. Br., pp. 84-97.

Kigoshi, K., 1971. Alpha recoil ${ }^{234} \mathrm{Th}$ : Dissolution in water and the ${ }^{234} \mathrm{U} /{ }^{238} \mathrm{U}$ disequilibrium in nature. Science, 173 , 47-48. 
Krishnaswami, S., Graustein, W.C., Turekian, K.K., Dowd, J.F., 1982. Radium, thorium and radioactive lead isotopes in groundwaters: Applications to the in-situ determination of adsorption-desorption rate constants and retardation factors. Water Resour. Res. 18 (6), 1633-1675.

Kölbel, L., Kölbel, T., Wiegand, B., Sauter, M., Schäfer, T., Siefert, D., 2020a Identification of fractured zones in geothermal reservoirs in sedimentary basins: A radionuclide-based approach. Geothermics, 85, 101764.

Kölbel, L., Kölbel, T., Wiegand, B., Sauter, M., Schäfer, T., Siefert, D., 2020b. Water-rock interactions in the Bruchsal geothermal system by U-Th series radionuclides. Submitted to Geothermal Energy on 2020-07-03.

Ku, T.-L., Luo, S., Leslie, B.W., Hammond, D.E., 1992. Decay-series disequilibria applied to the study of water-rock interaction and geothermal systems, in: Ivanovich, M., Harmon, R.S. (Eds.), Uranium-series Disequilibrium: Applications to Earth, Marine, and Environmental Sciences. Clarendon Press, Oxford, 631-668.

Luo, S., Ku, T.-L., Roback, R., Murrell, M., McLing, T.L., 2000 In-situ radionuclide transport and preferential groundwater flows at INEEL (Idaho): Decay-series disequilibrium studies. Geochim. Cosmochim. Acta, 64, 867881.

Mao, S., Duan, Z., 2008. The P, V, T,x properties of binary aqueous chloride solutions up to T = 573 Kand $100 \mathrm{MPa}$. J. Chem. Thermodynamics 40, 1046-1063.

Nazaroff W.W., Nero A.V., 1988. Radon and its decay products in indoor air. United States.

Osmond, J.K., Cowart, J.B., 1992. Ground water. In: Ivanovich, M., Harmon, R.S. (Eds.), Uranium-series Disequilibrium: Applications to Earth, Marine, and Environmental Sciences. Oxford Science Publications, pp. 290.

Pribnow, D., Schellschmidt, R., 2000. Thermal tracking of upper crustal fluid flow in the Rhine Graben. Geophysical Research Letters 27, 1957-1960.

Raghavan, R.,1977. Pressure behavior of wells intercepting fractures. Proc. Invitational Well-Testing Symposium, Berkeley, Oct. 19-21, 1977, Lawrence Berkeley Lab. Rep. LBL-7027 (Mar. 1977), 117-160.

Rama and Moore, W.S., 1984. Mechanism of transport of U-Th series radioisotopes from solids into ground water. Geochim. Cosmochim. Acta 48, 395-400.

Renard, P., Mejias, M., Glenz, D., 2009. Understanding diagnostic plots for well-test interpretation. Hydrogeological Journal, 17, 3, 589-600.

Sun, H., Semkow, T., 1998. Mobilization of thorium, radium and radon radionuclides in ground water by successive alpha-recoil. Journal of Hydrology 205, 126-136.

Tanner, A.B.,1964. Radon migration in the ground: a review. In: Adams, J.A.S. and Lowder, W.M. (eds), The Natural Radiation Environment, Chicago, 161-190.

Tricca, A., Wasserburg, G.J., Porcelli, D., Baskaran, M., 2001. The transport of U- and Th-series nuclides in a sandy unconfined aquifer. Geochim. Cosmochim. Acta 65, 1187-1210. 
Ziegler, P.A., 1992. European Cenozoic rift system. Tectonophysics 208, 91-111.

Zukin, J.G., Hammond, D.E., Ku, T.-L., Elders, W.A., 1987. Uranium-Thorium series radionuclides in brines and reservoir rocks from two deep geothermal boreholes in the Salton Sea geothermal field, southeastern California. Geochim. Cosmochim. Acta 51, 2719-2731. 


\section{Chapter VI}

\section{Conclusions}

\subsection{Integration and evaluation of methods of investigation and results}

A proper analysis of Th-U series nuclides may serve as an additional tool to tap geothermal energy resources starting from the exploration phase to reservoir enhancement and even during plant operation. Of particular interest are their contribution to (1) the reservoir engineering, (2) the plant operation and (3) the up-scaling of geothermal power plants. To assess these issues, different methods are reviewed in this thesis and applied on the geothermal test site in Bruchsal (Germany). The key outcomes of this examination are subsequently discussed.

\subsubsection{Detecting productive geothermal horizons while reservoir engi- neering}

A methodology for fracture zone identification in geothermal reservoirs based on Th- $\mathrm{U}$ isotope rock signatures was assessed in chapter 3. Performing alpha and gamma spectrometry surveys of drill cuttings from the Bruchsal wells revealed an accumulation of the long-lived ${ }^{226} \mathrm{Ra}$ in fractured zones. This is a key finding since radioactive equilibrium is to be expected for ${ }^{238} \mathrm{U}$ and its decay products due to the geological age of the rocks of about 250 million years. However, the enrichment of ${ }^{226} \mathrm{Ra}$ caused a disruption of the secular equilibrium of the uranium decay series. Within this decay series, the specific activity of ${ }^{226} \mathrm{Ra}$ exceeds the ${ }^{230} \mathrm{Th}$ activity by a factor of two to three, while the ${ }^{230} \mathrm{Th} /{ }^{234} \mathrm{U}$ and ${ }^{234} \mathrm{U} /{ }^{238} \mathrm{U}$ ratios are equal to unity, within an analytical error of one standard deviation, indicating that uranium has not been transported relative to thorium for the last $10^{5}$ $-10^{6}$ years.

Furthermore, it was found that the ${ }^{228} \mathrm{Ra} /{ }^{226} \mathrm{Ra}$ activity ratio is significantly lower in samples from deeper borehole sections than the expected ${ }^{228} \mathrm{Ra} /{ }^{226} \mathrm{Ra}$ ratio of 1.2 . From these results, the geothermal reservoir section could be limited to the depth interval $2330-2472 \mathrm{~m}$ corresponding to the Lower Buntsandstein and Zechstein units. 
Assuming that hydrothermal alteration processes resulting from the interaction between circulating geothermal fluid and the host rock characterize geothermal reservoirs, a petrographic alteration study was additionally performed. Radionuclide signatures associated with secondary alteration processes in the rock samples allowed the identification of possible water inflow zones. To validate these results, geophysical well logs of GB2 were used.

Within the open-hole section of the production well GB2, five possible fractured zones were identified by the evaluation of $\mathrm{Th} / \mathrm{Ra}$ and $\mathrm{Ra} / \mathrm{K}$ ratios which are sensitive to changes in the ${ }^{226} \mathrm{Ra}$ concentration. Four of the zones were detected by different logging tools such as natural gamma-ray, resistivity, temperature, and caliper logs. There are indications that some zones are sealed by the precipitation of secondary minerals. This is a limitation for this method because it is not possible to distinguish between recent and paleo-fractures solely by radionuclide signatures. Nevertheless, the investigation of radionuclide distributions in natural reservoirs could be regarded as a supplementary method to define productive geothermal horizons. They can be analyzed and interpreted with some acceptable delay while drilling operations are in progress, or at a later stage in case other methods fail to provide the necessary information.

\subsubsection{Assessing Th-U migration behavior during power plant operation}

The migratory behavior of Th-U series radionuclides is examined in chapter 4. A comprehensive dataset was generated by regular, long-term fluid sampling at Bruchsal. Chemical and radiochemical investigations were conducted on thermal water abstracted from the production well GB2 during fluid circulation. In detail, isotopes of $\mathrm{Ra}\left({ }^{226} \mathrm{Ra},{ }^{228} \mathrm{Ra},{ }^{224} \mathrm{Ra},{ }^{223} \mathrm{Ra}\right), \mathrm{Rn}\left({ }^{222} \mathrm{Rn}\right)$ and $\mathrm{Pb}\left({ }^{210} \mathrm{~Pb},{ }^{212} \mathrm{~Pb}\right)$, $\mathrm{U}\left({ }^{238} \mathrm{U},{ }^{234} \mathrm{U},{ }^{235} \mathrm{U}\right)$, Th $\left({ }^{232} \mathrm{Th},{ }^{228} \mathrm{Th},{ }^{230} \mathrm{Th}\right)$, Po $\left({ }^{210} \mathrm{Po}\right)$ and $\mathrm{Ac}\left({ }^{227} \mathrm{Ac},{ }^{228} \mathrm{Ac}\right)$ were analyzed.

A model based on the interaction model suggested by Ku et al. (1992) was established using Mathcad ${ }^{\circledR}$, a numerical software with computer algebra system (CAS) capabilities. In order to check the CAS approach, MIN3P, a multicomponent reactive transport code, was applied (Mayer at al., 2002).

The results indicate that radionuclides of the elements radium, radon, and lead are rather soluble in brine, while isotopes of uranium, thorium, polonium, and actinium have low solubilities and are rather immobile. Activities of radium isotopes in the geothermal brine exceed those of their thorium progenitors resulting in radioactive disequilibria.

Modeling the disequilibria based on radium $\left({ }^{226} \mathrm{Ra},{ }^{228} \mathrm{Ra},{ }^{224} \mathrm{Ra},{ }^{223} \mathrm{Ra}\right)$ allowed the estimation of rate constants of water-rock interactions. Since the daughter-parent ratio of ${ }^{222} \mathrm{Rn} /{ }^{226} \mathrm{Ra}$ is ca. $1.3 \mathrm{Ra}$ retardation due to sorption processes is small. Precipitation occurs on timescales comparable to ${ }^{226} \mathrm{Ra}$ and ${ }^{228} \mathrm{Ra}$, while the short-lived ${ }^{224} \mathrm{Ra}$ and ${ }^{223} \mathrm{Ra}\left(\mathrm{t}_{1 / 2}=3.66\right.$ and 11.43 days, respectively) are not affected. Indeed, the short-lived $\mathrm{Ra}$ isotopes are mostly supplied from alpha recoil. 
The observed decay-series disequilibria in the Bruchsal brine can be best explained by the following conclusions on water-rock interaction processes:

(1) The hydraulically inactive pore spaces and microfractures, respectively, are the main source for alpha recoil, while recoil supply from large fractures may be negligible due to their relatively low brine-rock interaction rates.

(2) Diffusional flux through pore spaces and microfractures is believed to supply radionuclides to the large conductive fractures causing a significant fractionation of $\mathrm{Ra}$ isotopes.

(3) Migration through microfractures may limit input of short-lived Ra isotopes into solution, depending on isotope half-lives and fracture geometry.

(4) Radium removal is rather by co-precipitation with solid solutions than by sorption processes which is indicated by the isotopic ratio of ${ }^{222} \mathrm{Rn} /{ }^{226} \mathrm{Ra}=1.3$ in brine.

(5) Co-precipitation of radium is most likely for barite and restricted to the fractured reservoir section.

Consequently, the model approach suggested by Ku et al. (1992) has been successfully adapted to the site-specific conditions in Bruchsal. Using naturally occurring $U$ and Th decay-series disequilibria as a tool to evaluate the in-situ radionuclide migration in the natural systems offers a high benefit for the sustainable management of geothermal reservoirs.

\subsubsection{Up-scaling of geothermal power plants}

An integrated approach to estimate site-specific fracture dimensions (such as fracture width, length, etc.) based on a ${ }^{222} \mathrm{Rn}$ mass balance approach is considered in chapter 6 . The model approach is based on the research results presented in chapter 3 and chapter 4 . In addition, hydraulic reservoir parameters were obtained from well testing data performed in the past.

The evaluation of the flow regime indicated a fracture dominated fluid flow without further matrix support at Bruchsal site. Diffusional flux through microfractures acting as diffusion pathways is believed to supply ${ }^{222} \mathrm{Rn}$ in larger conductive fractures in which the sampled brine resides. Thus, the activity of ${ }^{222} \mathrm{Rn}$ dissolved within the large fracture fluid depends on the diffusional flux from rock surfaces and the size of the fracture whereas the diffusion length $\left(L=\sqrt{D_{m} / \lambda}\right)$ depends on the ${ }^{222} \mathrm{Rn}$ decay constant $\lambda_{\mathrm{R} n 2}\left(\mathrm{~s}^{-1}\right)$ and the diffusion coefficient $D_{m}\left(\mathrm{~cm}^{2} \mathrm{~s}^{-1}\right)$. The ${ }^{222} \mathrm{Rn}$ flux $\left(F_{\mathrm{R} n 2}\right)$ from planar rock surfaces (atoms s${ }^{-1} \mathrm{~cm}^{-2}$ ) into the fracture fluid by diffusion was determined using the model approach of Ku et al. (1992). Site-specific fracture width was derived from the ratio of ${ }^{222} \mathrm{Rn}$ input terms $\left(F_{\mathrm{R} n 2}+P_{r}\right)$ to the dissolved radon activity produced by diffusional flux. 
The fracture length connected to the Bruchsal production well was estimated by means of both, the one-dimensional transport modeling of ${ }^{222} \mathrm{Rn}$ and hydraulic simulations applying the mathematical solution of Gringarten and Witherspoon (1972) for infinite flow in vertical fractures. The fracture length is related to the specific storage coefficient where the relation between both parameters can be described by the function $\left(\mathrm{L}_{\mathrm{f}}^{2} \mathrm{x} \mathrm{S} \approx\right.$ const.).

Estimates about the specific surface area $\left(\mathrm{m}^{2} \mathrm{~m}^{-3}\right)$ was deduced from the known flux $\left(F_{\mathrm{R} n 2}\right)$ and the fluid activity $\left(A_{R_{n}}\right)$ of radon. In this case, the specific surface area is defined as the surface area of solids per volume of fracture fluid contacting the solids (which corresponds to the flow wetted surface area). Since the specific surface area of the fracture is inversely proportional to the fracture width, further information about the site-specific fracture geometry was obtained.

However, it should be noted that the fracture width estimated for the Bruchsal geothermal system represents an averaged equivalent aperture since the fluid samples analyzed are mostly collected from the larger aperture, hydraulically conductive fractures. Thus, the fracture surface area depends on (a) whether the estimated fracture width represents one large or several smaller fractures and (b) the extent of the variance from the averaged equivalent fracture width.

\subsection{Limits of application}

The methodological approach of this work uses Th-U series disequilibria for the characterization of deep geothermal reservoirs. As discussed in the previous chapters, certain prerequisites have to be met for the application. A summary of the key points is listed below.

The radionuclide-based approach used for the identification of fractured zones in geothermal reservoir section is based on the premise that radioactive disequilibria not only occurs in the fluids, but also in the interacting solids. This requirement is not in agreement with each natural system because radionuclides usually have much longer residence times in solid phases than in solution and thus, more time to achieve a state of radioactive equilibrium.

Fractured and hydrothermally altered horizons of the Bruchsal geothermal reservoir are associated with a preferential accumulation of ${ }^{226} \mathrm{Ra}$ in the solid phase causing a measurable disruption of the secular equilibrium of the uranium decay series. However, the method is limited in such a way that it is not possible to distinguish between recent and paleo-fractures solely by radionuclide signatures.

The water-rock interaction model presented is applicable to fluid systems being a) in a state of radioactive disequilibria and b) in excess of dissolved radium. These systems are not closed (fluid phases are interacting with the adjoining solids) and have physico-chemical conditions (salinity, temperature, and redox state, etc.) encouraging the transport of radium isotopes in solution. Such conditions are usually found in deep saline formation waters with relatively high temperatures. A 
limited applicability is given to low salinity environments where radium is strongly adsorbed on solids surface. Even though the model presents a quite comprehensive approach of water-rock interaction, there remain a number of assumptions and simplifications concerning, inter alia, the inhomogeneities in solid phase distribution, the nature of alpha recoil input and the geometry of fractures.

Fractured reservoirs are extremely heterogeneous considering their hydraulic characteristics. For that reason, using Th-U series nuclides for the estimation of site-specific fracture dimensions requires a model of an equivalent fracture as implemented in the model of Gringarten and Witherspoon (1972) that approximates the hydraulic behavior of a naturally fractured system. This approach restricts the range of application to natural systems with fracture-dominated fluid flow without further matrix support. Furthermore, the application of the joint inversion of the radon diffusion model combined with pumping test results is not without interpretative hydraulic parameter ambiguity. For example, fracture length is related to the storage coefficient whereby the fracture length may differ several hundred meters by changing the specific storage coefficient within one order of magnitude.

\subsection{Perspectives}

The results presented in this thesis indicate that a differentiated analysis of thorium and uranium decay series radionuclides are suitable to provide useful information about fracture parameters. Although the understanding of the site-specific behavior of Th- $\mathrm{U}$ series disequilibria has been highly improved by this study, a certain number of details remain to be examined.

(1) The discrimination between hydraulically active and non-active fractures cannot be achieved by the radionuclide signatures alone as these fractures can also be sealed by precipitates of hydrothermal minerals. Therefore, the behavior of previously dissolved radium needs to be addressed in additional studies. From a mineralogical point of view, both co-precipitation in sulphate minerals/carbonates as well as adsorption on clay minerals/metal hydroxides are potentially responsible for radium scavenging from the geothermal fluid. However, these processes have not been fully examined yet. Laboratory studies of the solids and their associated fluids (e.g. leaching experiments) may offer an opportunity to improve the understanding of this specific water-rock interaction. In addition, it is an advantage of radionuclide signature analysis based on drill cuttings that the sample transfer to a laboratory as well as preparation and analysis is not time expensive. It seems to be promising to combine this method with result from logging while drilling technique (LWD) in a joint evaluation and interpretation.

(2) The interaction model developed within the framework of this thesis is only tested at the 
geothermal system in Bruchsal. Since boundary conditions and assumptions of the water-rock interaction used in this model reflecting the chemistry and physical state of the Bruchsal site, further effort needs to be spent on the question to what extent the presented diffusion flux model can be applied at other geothermal sites. Such investigations at existing geothermal sites in the Upper Rhine Graben might be an appropriate next step because of the comparable fluid chemistry (Aquilina et al., 1997) and reservoir conditions (Vidal and Genter, 2018).

(3) Since the fluid samples are mostly collected from the larger conductive fractures, estimates of the fracture width represents an averaged equivalent fracture width. Whether or not the fracture aperture depends on one large or several smaller fractures is of interest for the design of well cleaning operations, e.g. chemical stimulation. This challenging question could be addressed by further improvement of the ${ }^{222} \mathrm{Rn}$ mass balance approach as suggested by Andrews et al., (1986) and others. A combination of the method presented here with other techniques can provide significant support to resolve the ambiguity. A promising approach may be the application of tracer tests, particularly push-and-pull tests as extensively discussed in Ghergut at al. (2013).

\section{References}

Andrews J.N., Hussain N., Batchelor A.S., Kwakwa K., 1986. 222Rn solution by the circulating fluids in a 'hot dry rock' geothermal reservoir. Appl. Geochim. 1, 647-657.

Aquilina, L., Pauwels, H., Genter, A., Fouillac, C., 1997. Water-rock interaction processes in the Triassic sandstone and the granitic basement of the Rhine Graben: Geochemical investigations of a geothermal reservoir, in: Geochimica et Cosmochimica Acta, 61, S. 4281-4295.

Ghergut, I., Behrens, H., Sauter, M., 2013. Single-well tracer push-pull test sensivitiy to fracture aperture and spacing. In: Proceedings of the 38th Workshop on Geothermal Reservoir Engineering; Stanford University, Stanford, California, February 11-13, 2013.

Gringarten, A.C., Witherspoon, P.A., 1972. A method of analyzing pump test data from fractured aquifers, Int. Soc. Rock Mechanics and Int. Assoc. Eng. Geol., Proc. Symp. Rock Mechanics, Stuttgart, Vol. 3B, 1-9.

Ku, T.-L., Luo, S., Leslie, B.W., Hammond, D.E., 1992. Decay-series disequilibria applied to the study of water-rock interaction and geothermal systems, in: Ivanovich, M., Harmon, R.S. (Eds.), Uranium-series Disequilibrium: Applications to Earth, Marine, and Environmental Sciences. Clarendon Press, Oxford, 631-668.

Mayer, K.U., Frind, E.O., Blowes, D.W., 2002. Multicomponent reactive transport modeling in variably saturated porous media using a generalized formulation for kinetically controlled reactions. Water Resour. Res. 38, 1174.

Vidal, J., Genter, A., 2018. Overview of naturally permeable fractured reservoirs in the central and southern Upper

Rhine Graben: Insights from geothermal wells. Geothermics 74, 57-73. 